

\section{WHOI $-77-4$}

DATA REPORT FOR ATLANTIC PELAGIC ZOOGEOGRAPHY

\section{by}

Richard H. Backus and James E. Craddock

WOODS HOLE OCEANOGRAPHIC INSTITUTION
WOOds Hole, Massachusetts 02543

January 1977

TECHNICAL REPORT

Prepared for the National Science Foundation under Grant DES 74-23209.

Reproduction in whole or in part is permitted for any purpose of the United States Government. In citing this manuscript in a bibliography, the reference should be followed by the phrase: UNPUBLISHED MANUSCRIPT.

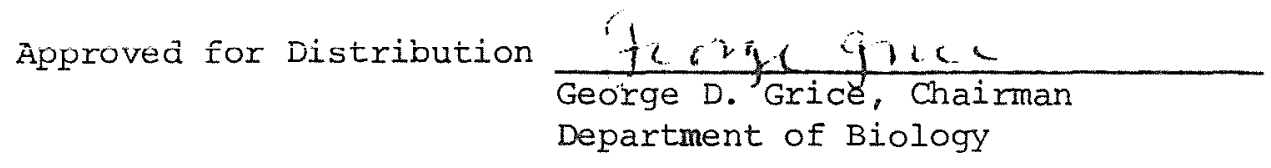




\section{DATA REPORT FOR ATLANTIC PELAGIC ZOOGEOGRAPHY}

Richard H. Backus and James E. Craddock

This data report fulfils two functions. It (1) gives station data for 1022 midwater trawl collections made in the Atlantic Ocean between 1961 and 1974 by the writers and their colleagues (Table 1, Figure 1, and Appendix 1) and for 531 Atlantic neuston collections made between 1964 and 1974 (Table 2 and Appendix 2), and (2) gives the geographic coordinates for a set of boundaries that divides the Atlantic Ocean between the arctic-subarctic boundary and the subtropical convergence at $40^{\circ} \mathrm{S}$ into a system of faunal regions and provinces (Figure 2 and Appendix 3). The derivation of these boundaries is explained briefly. More details can be found in the last of the papers and manuscripts listed immediately below, which are based on fish data from the collections listed and relate to the system of faunal boundaries described.

Backus, R. H., G. W. Mead, R. L. Haedrich, and A. W. Ebeling. 1965. The mesopelagic fishes collected during Cruise 17 of the $\mathrm{R} / \mathrm{V}$ CHAIN, with a method for analyzing faunal transects.' Bull. Mus. Comp. Zoo1. 134: 139-158.

Backus, R. H., J. E. Craduock, R. L. Haedrich, and D. L. Shores. 1969. Mesopelagic fishes and thermal fronts in the western Sargasso Sea. Mar. Biol. 3: 87-106. 
Backus, R. H., J. E. Craddock, R. L. Haedrich and D. L. Shores. 1970. The distribution of mesopelagic fishes in the equatorial and western North Atlantic Ocean. J. Mar. Res. 28: 179-201.

Backus, R. H. 1972. Midwater fish distribution and sound-scattering levels in the North Atlantic Ocean. U. S. Navy J. Underwater Acoust. 22: 243-255.

Jahn, A. E. and R. H. Backus. 1976. On the mesopelagic fish faunas of Slope Water, Gulf Stream, and Northern Sargasso Sea. DeepSea Res. 23: 223-234.

Backus, R. H. and J. E. Craddock. In press. Pelagic fauna1 provinces and sound-scattering levels in the Atlantic Ocean. In Oceanic sound-scattering prediction, N. R. Andersen and B. J. Zahuranec [eds.], Marine Science, vo1. 3. Plenum Press. Nafpaktitis, B. G., R. H. Backus, J. E. Craddock, R. L. Haedrich, B. H. Robison and C. Karnella. In press. Family Myctophidae. In R. H. Gibbs, Jr. [ed..], Fishes of the western North Atlantic, pt. 7. Mem. Sears Found. Mar. Res. 1. Allen Press. 
Table 1. Summary of midwater trawl collections.

\begin{tabular}{|c|c|c|c|}
\hline Cruise & Date & Area & $\begin{array}{c}\text { Number } \\
\text { of } \\
\text { Collections }\end{array}$ \\
\hline Chain 17 & Apr-May 1961 & $\begin{array}{l}\text { Equatorial Atlantic into } \\
\text { Sargasso Sea }\end{array}$ & 18 \\
\hline Chain 32 & Sept 1962 & Slope Water Region & 19 \\
\hline Chain 35 & Feb-Mar 1963 & Equatorial Atlantic & 31 \\
\hline Atlantis II 13 & Sept-Oct 1964 & Woods Hole to Azores & 52 \\
\hline Chain 49 & June 1965 & Caribbean; Sargasso Sea & 31 \\
\hline Atlantis II 20 & Feb-Apr 1966 & Equatorial Atlantic & 24 \\
\hline Chain 60 & May-June 1966 & Caribbean; Gulf of Mexico & 68 \\
\hline Atlantis II 31 & Feb-Mar 1967 & Western South Atlantic & 23 \\
\hline Chain 72 & Aug 1967 & Slope Water into Sargasso Sea & 25 \\
\hline Gosnold 106 & Oct 1967 & Slope Water & 4 \\
\hline Gosnold 135 & Oct 1968 & Slope Water & 1 \\
\hline Chain 85 & Nov-Dec 1968 & Sargasso Sea & 39 \\
\hline Atlantis II 49 & May-June 1969 & Mediterranean; Eastern Atlantic & 143 \\
\hline Atlantis II 59 & Oct-Dec 1970 & Eastern Atlantic & 119 \\
\hline Atlantis II 60 & Apr-June 1971 & South Atlantic & 99 \\
\hline Knorr 24 & Nov 1971 & Mediterranean & 18 \\
\hline Chain 105 & June-July 1972 & Northern North Atlantic & 145 \\
\hline Walther Herwig & Sept 1973 & Eastern North Atlantic & 10 \\
\hline Atlantis II 78 & Sept-Oct 1973 & Azores to Equatorial Atlantic & 121 \\
\hline Atlantis II 79 & Dec 1973 & Caribbean Sea & 7 \\
\hline Knorr 38 & Mar 1974 & Sargasso Sea & 25 \\
\hline Total & & & 1,022 \\
\hline
\end{tabular}


Table 2. Summary of neuston collections.

\begin{tabular}{|c|c|c|c|}
\hline Cruise & Date & Area & $\begin{array}{c}\text { Number } \\
\text { of } \\
\text { Collections }\end{array}$ \\
\hline Atlantis II 13 & Sept-Oct 1964 & New England-Azores & 15 \\
\hline Chain 49 & June 1965 & Sargasso Sea & 15 \\
\hline Atlantis II 20 & Feb-Apr 1966 & Equatorial Atlantic & 13 \\
\hline Chain 60 & May-June 1966 & Gulf of Mexico; Caribbean Sea & 24 \\
\hline Atlantis II 31 & Feb-Mar 1967 & Western South Atlantic & 12 \\
\hline Chain 72 & Aug 1967 & Slope Water & 12 \\
\hline$\overline{\text { Gosnold } 106}$ & Oct 1967 & Slope Water & 5 \\
\hline Chain 75 & Oct-Nov 1967 & $\begin{array}{l}\text { Eastern Caribbean Sea } \\
\text { Southern Sargasso Sea }\end{array}$ & 17 \\
\hline Gosnold 121 & June 1968 & Slope Water & 2 \\
\hline$\overline{\text { Gosnold }} 122$ & July 1968 & Slope Water & 4 \\
\hline$\overline{\text { Crawford }} 172$ & Aug 1968 & Slope Water & 11 \\
\hline$\overline{\text { Gosnold }} 135$ & Oct 1968 & Slope Water & 3 \\
\hline$\overline{\text { Chain } 85}$ & Nov-Dec 1968 & Sargasso Sea & 27 \\
\hline 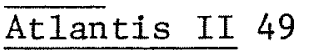 & May-June 1969 & Mediterranean, Eastern Atlantic & 92 \\
\hline$\overline{\text { Gosnold } 148}$ & Sept 1969 & Slope Water & 3 \\
\hline Atlantis II 59 & Oct-Dec 1970 & Eastern Atlantic & 66 \\
\hline Atlantis II 60 & Apr-June 1971 & South Atlantic & 63 \\
\hline Lu1u 3-71 & June 1971 & Slope Water & 11 \\
\hline Chain 105 & June-July 1972 & Northern North Atlantic & 55 \\
\hline Atlantis II 78 & Sept-Oct 1973 & Azores to Equatorial Atlantic & 67 \\
\hline Knorr 38 & Mar 1974 & Sargasso Sea & 14 \\
\hline Tota1 & & & 531 \\
\hline
\end{tabular}


Table 3. Atlantic Ocean faunal regions and provinces.

I. Atlantic Subarctic Region

1 Atlantic Subarctic Province

II. North Atlantic Temperate Region

2 Northern Gyre

3 Slope Water

4 Azores-Britain Province

5 Mediterranean Outflow

6 Western Mediterranean Sea

7 Eastern Mediterranean Sea

III. North Atlantic Subtropical Region

8 Northern Sargasso Sea

9 Southern Sargasso Sea

10 Northern North African Subtropical Sea

11 Southern North African Subtropical Sea

IV. Gulf of Mexico (Region)

12 Gulf of Mexico (Province)

V. Mauritanian Upwelling

18 Northern Mauritanian Upwelling

19 Southern Mauritanian Upwelling

VI. Atlantic Tropical Region

12 Lesser Antillean Province

14 Caribbean Sea

15 Amazonian Province

16 Guinean Province

23 Straits of Florida

VII. South Atlantic Subtropical Region

17 South Atlantic Subtropical Sea 


\section{Figure Captions}

Figure 1 - WHOI midwater traw1 collections, 1961-1974.

Figure 2 - Atlantic Ocean faunal regions and provinces. The regions are named, the provinces numbered in accordance with Table 3 . 


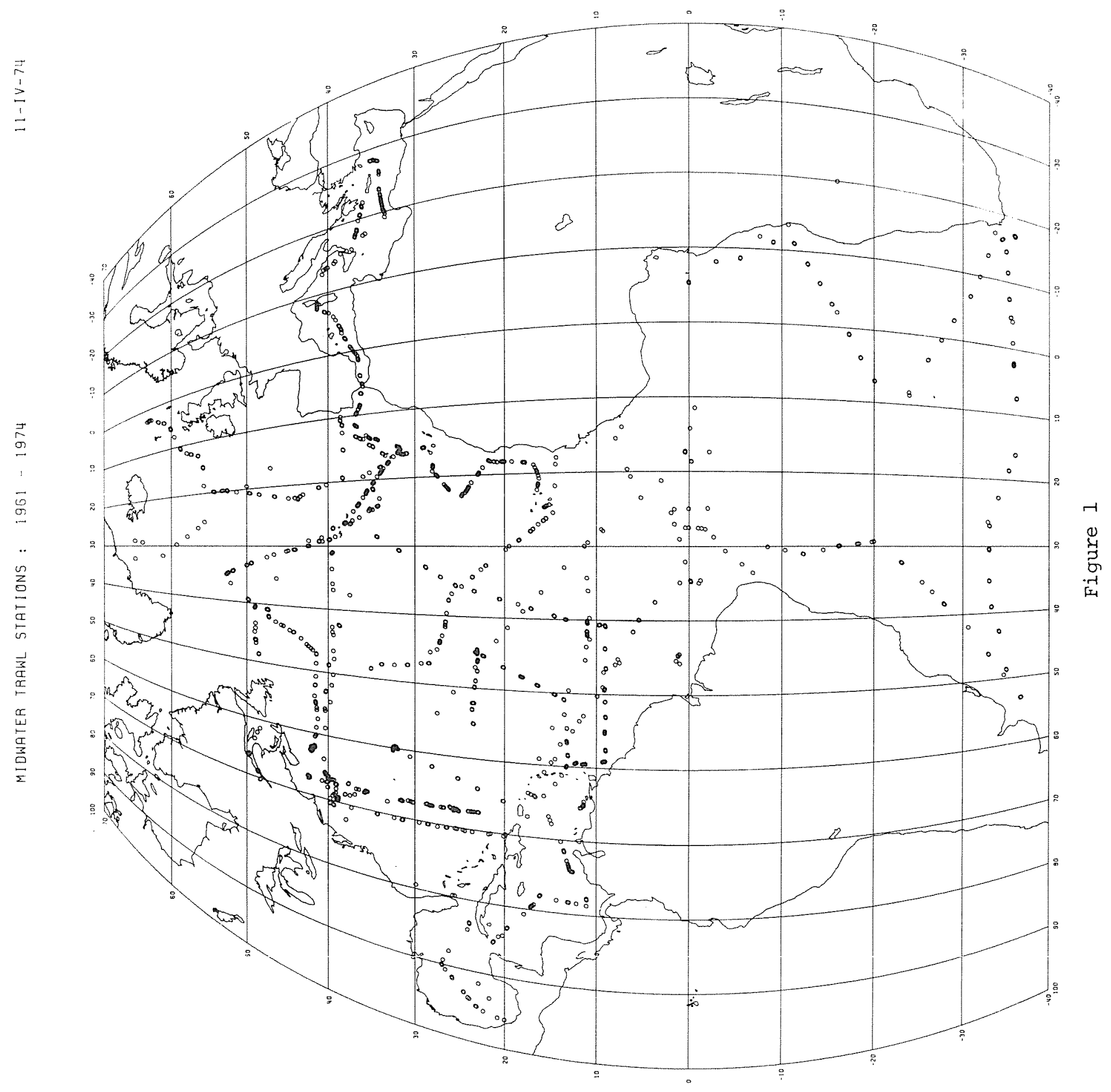




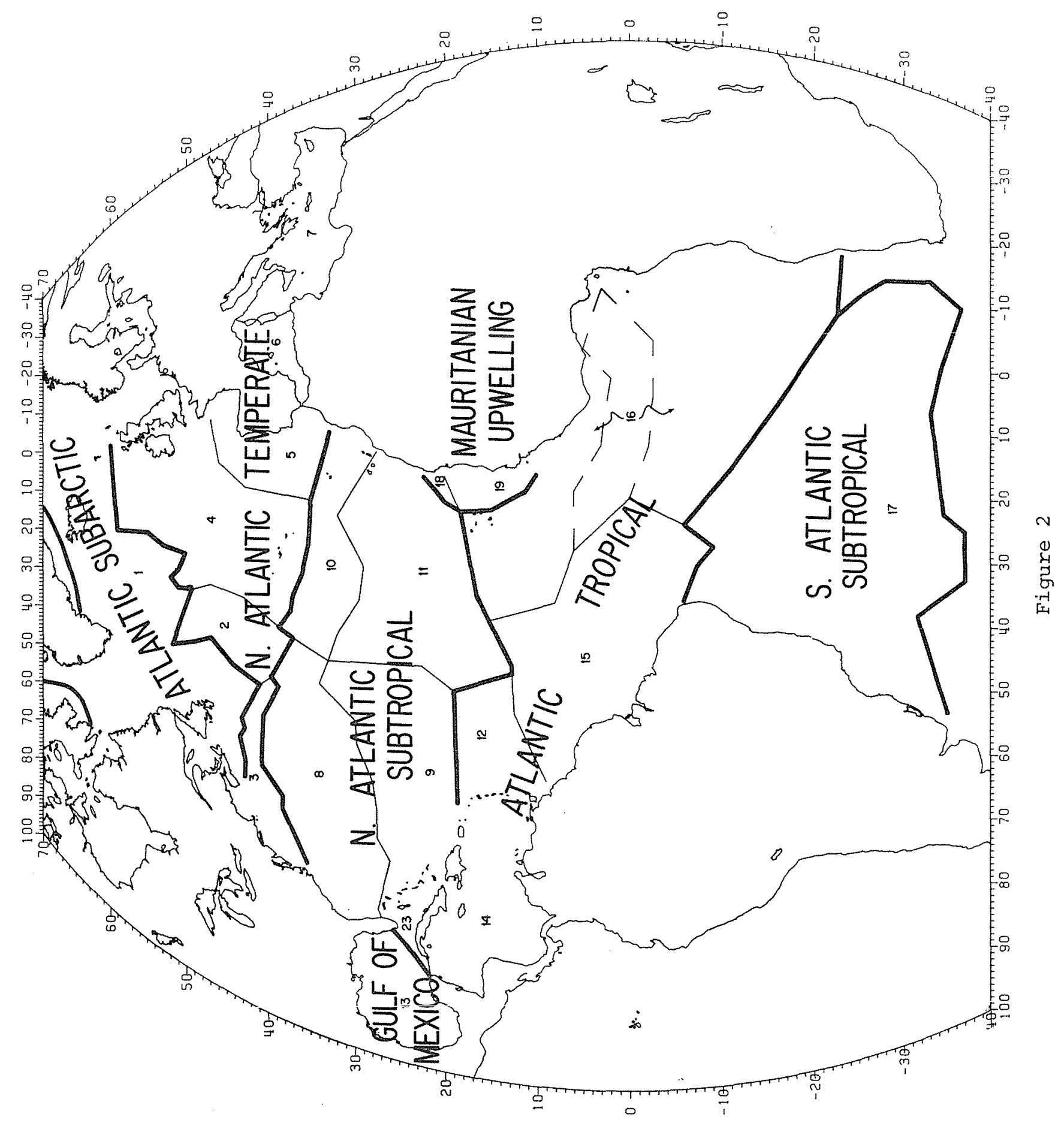




\author{
Appendix 1 \\ WHOI Midwater Traw1 Collection Data, 1961-1974.
}

Under the column "SHIP", $\mathrm{CH}=$ Chain, $\mathrm{A} 2$ = Atlantis $I I, \mathrm{GO}=$ Gosnold, $\mathrm{KN}=\underline{\text { Knorr }}$, and $\mathrm{WH}=$ Walther $\underline{\text { Herwig. }}$. The Walther Herwig collections (RHB 2800-2809) were made on an OVERFLOW 73 cruise, and the numbers shown under "CRUISE" for these collections are Walther Herwig station numbers. Collection depth is in meters and shows the stratum principally fished. Temperatures are in degrees Centigrade. Under "GEAR", 10IKT $=10$-foot Isaacs-Kidd midwater trawl, 3IKT $=3$-foot Isaacs-Kidd midwater trawl, and MMT = Marinovich midwater traw1, an experimental net we used briefly; none used opening-closing devices. Under "CODE", $\mathrm{D}=$ day, $\mathrm{N}=$ night, and $\mathrm{T}=$ twilight. The numbers under "PROV." refer to the faunal provinces in Table 3, but not found there are provinces 20,21 , and 22 , which were assigned to tentative provinces in the South Atlantic at the extremity of our collecting. 


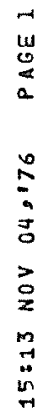

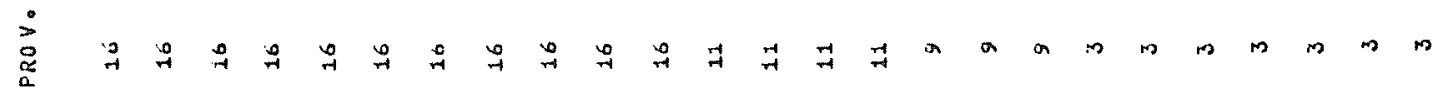
号

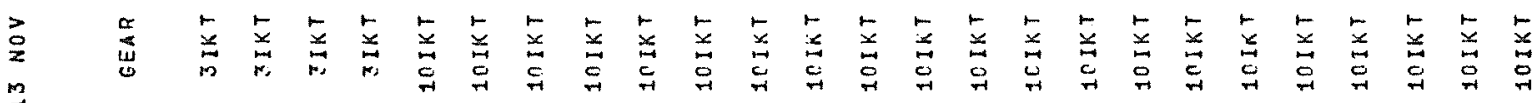

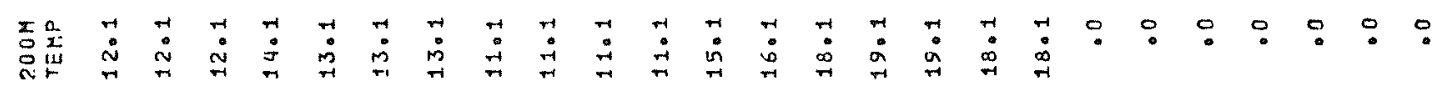

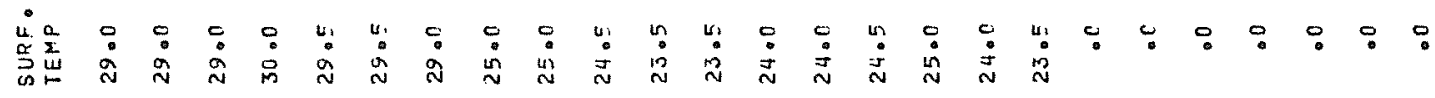

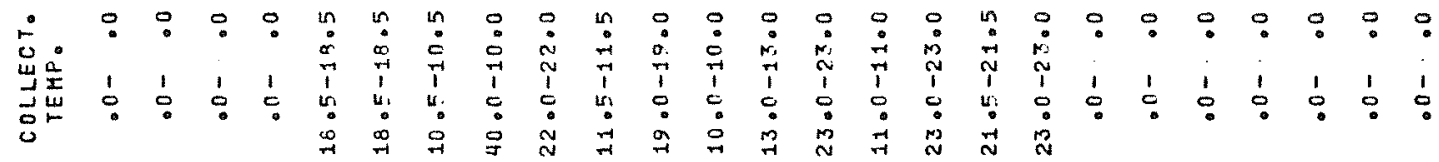

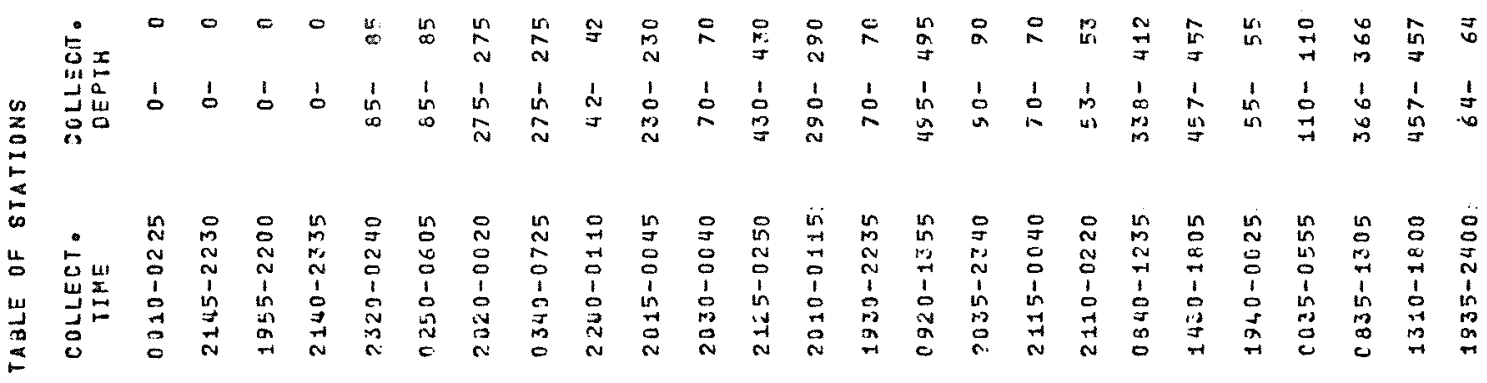

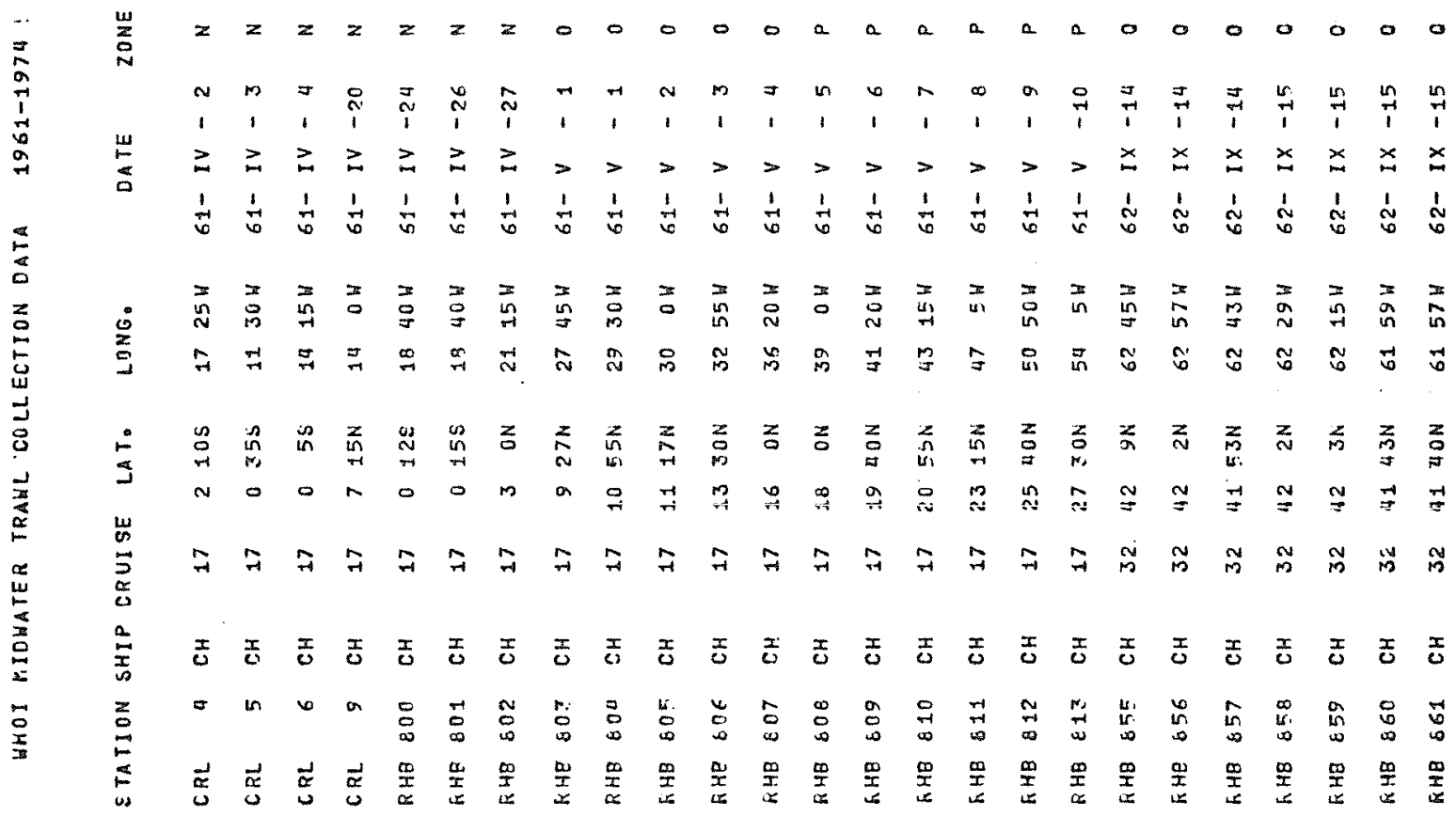




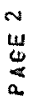

$$
\dot{\vec{x}}
$$

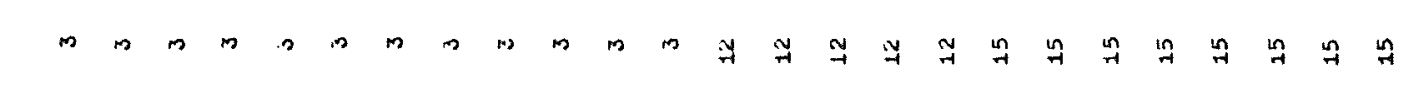

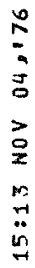

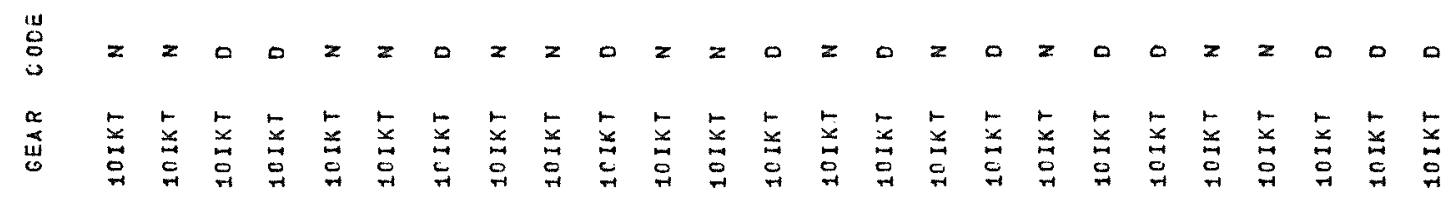
焉: : : : : : : : : : : : : : : : : : : : :

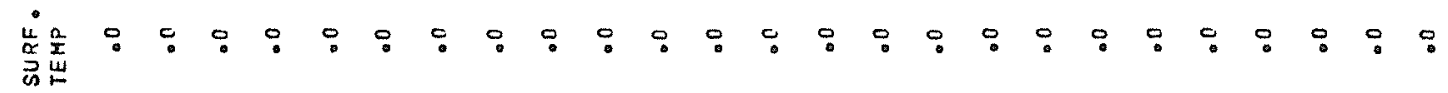

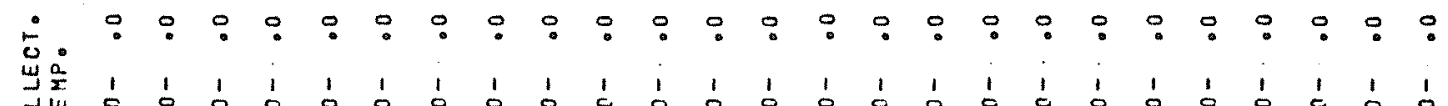
岁热 1

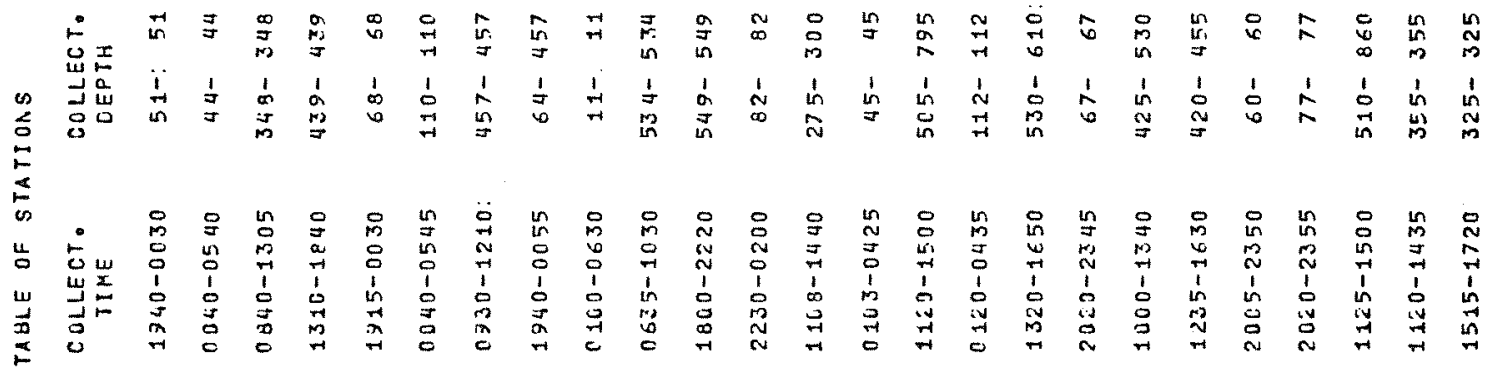

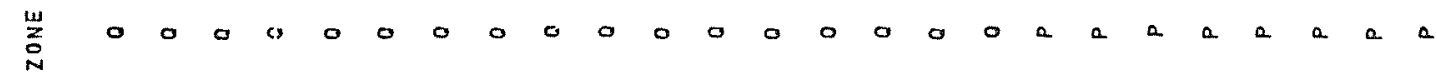
जิ

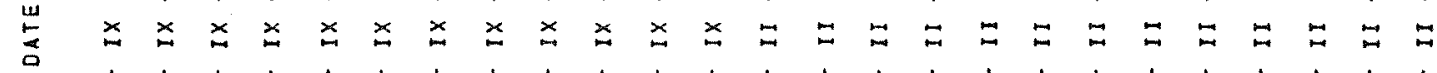
委

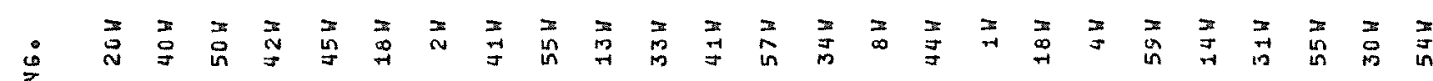
尊

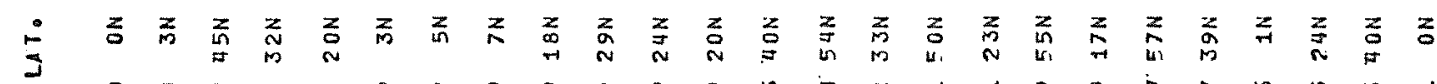

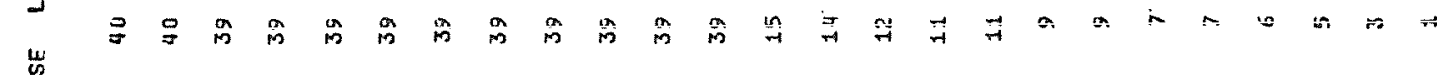

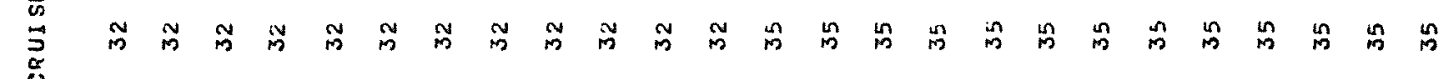
隺

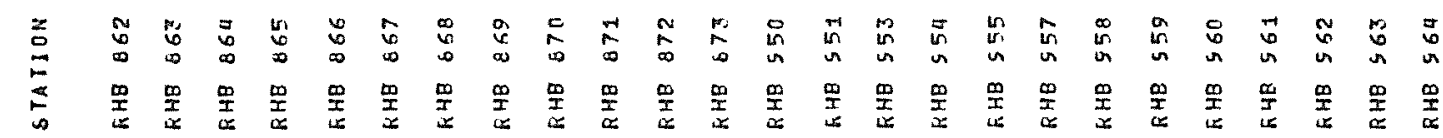




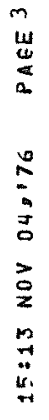

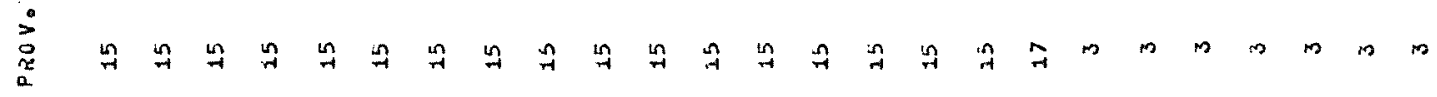

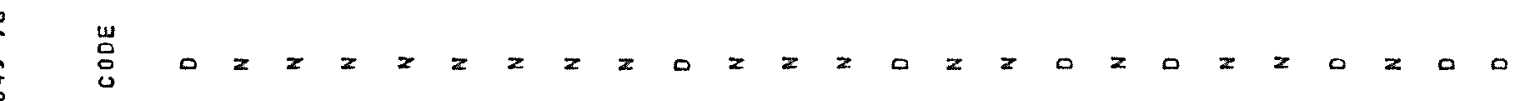

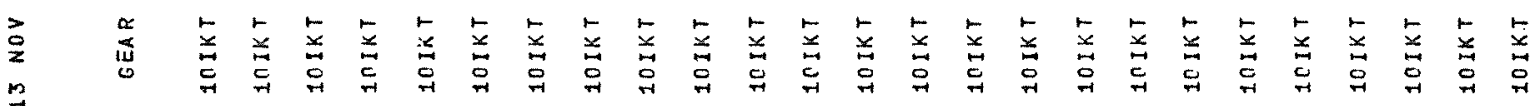

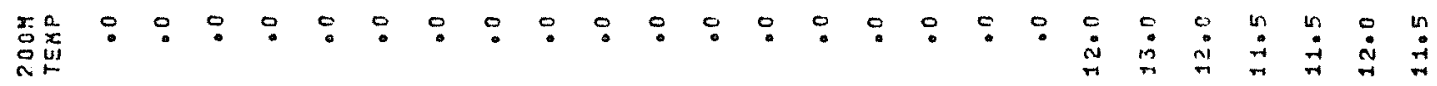

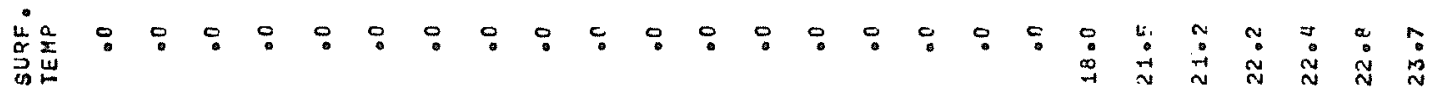

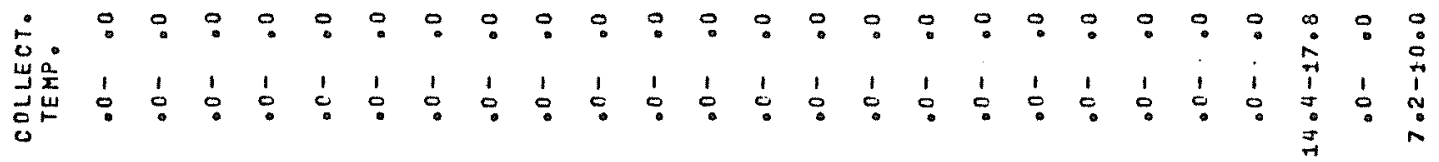

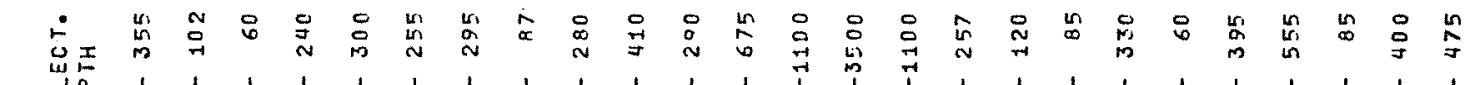

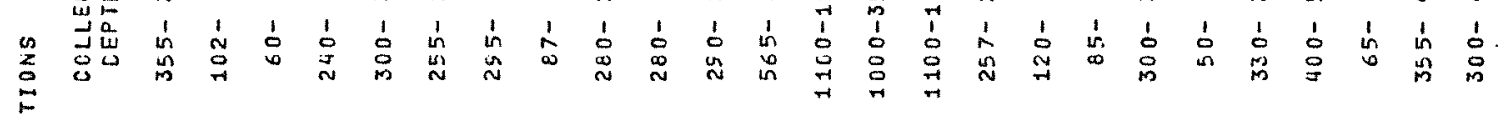
5

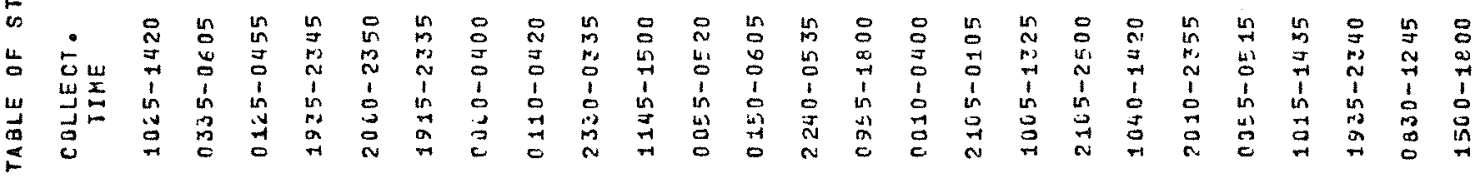
â

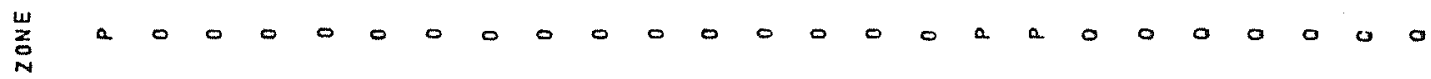

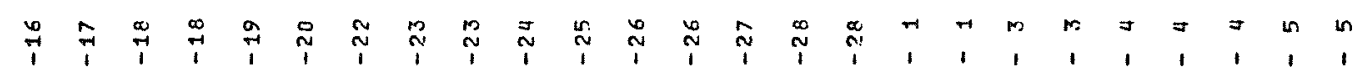

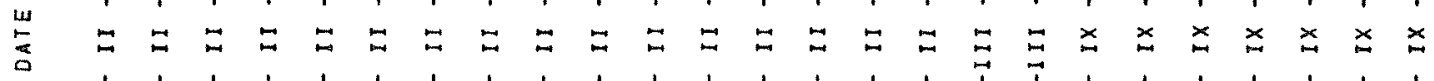

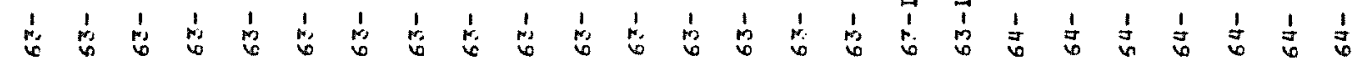




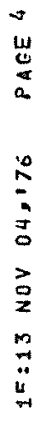

$\underset{\substack{x \\ a}}{\dot{x}}$

iे

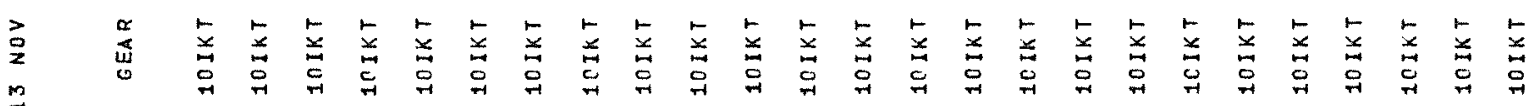

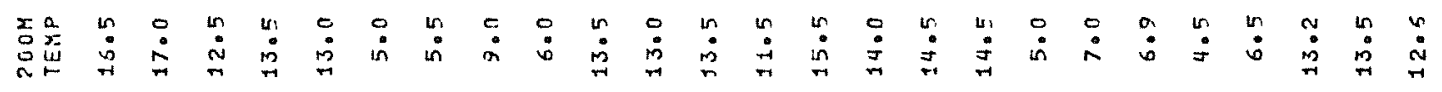

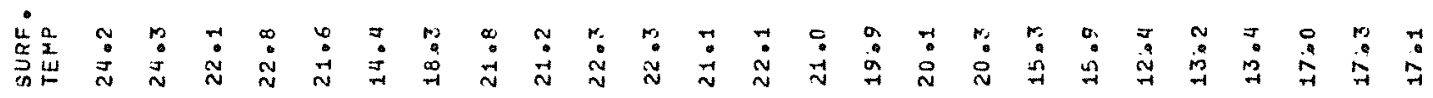

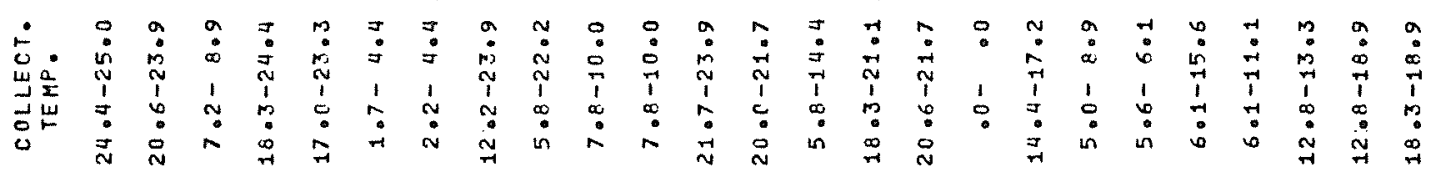

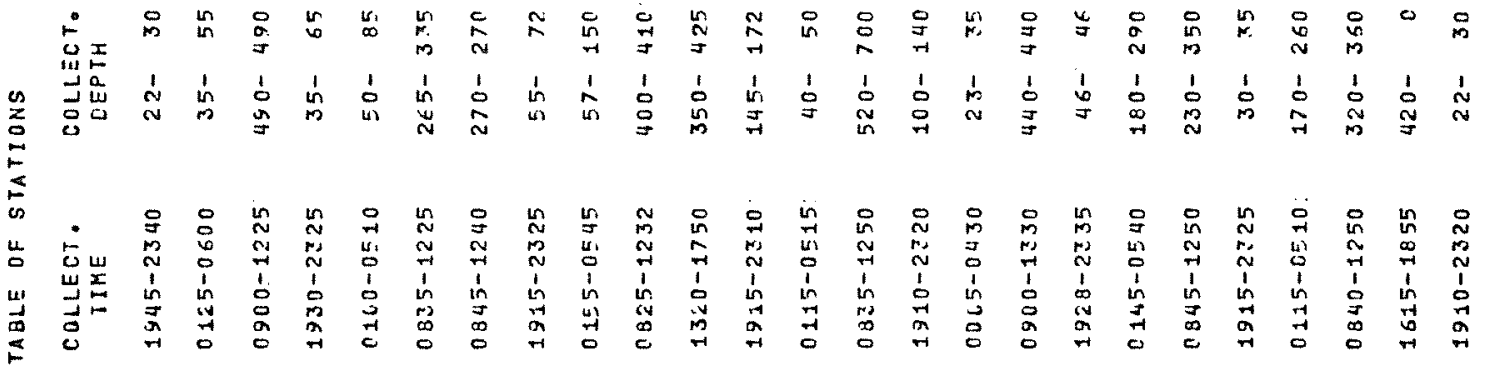

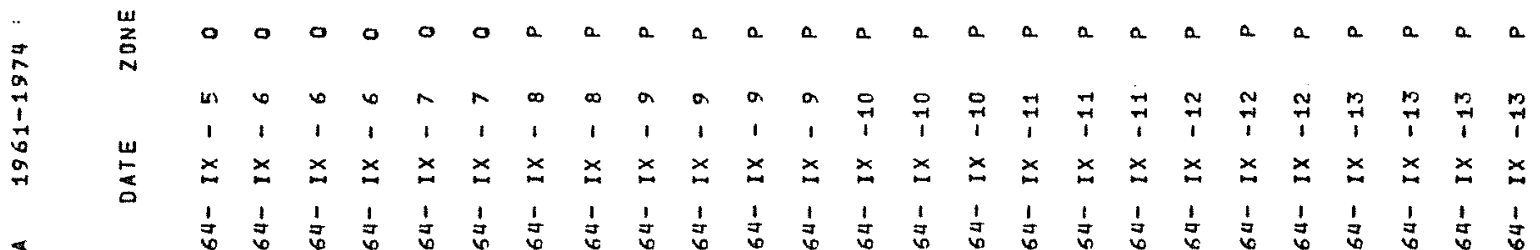

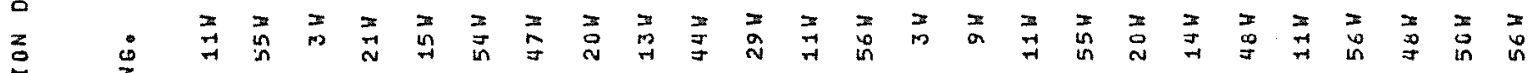

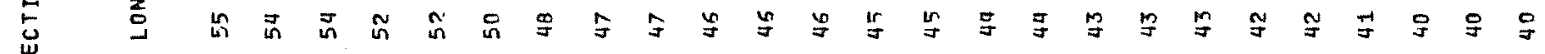

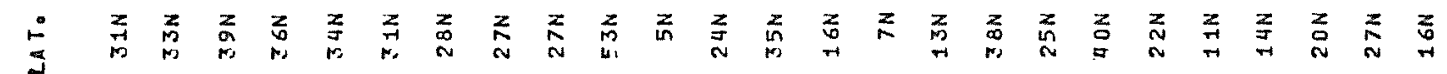
焉

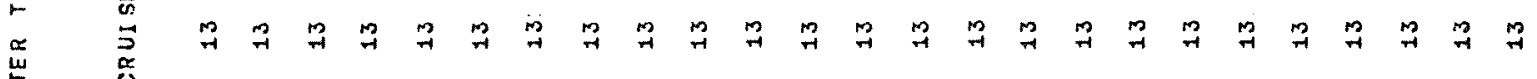

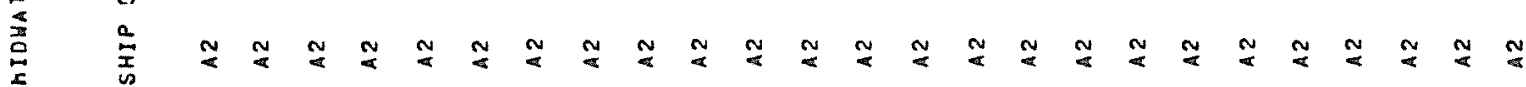

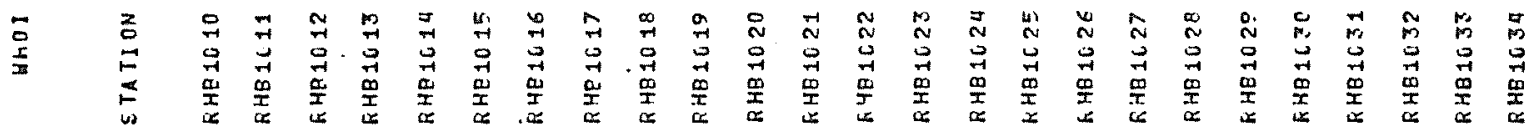




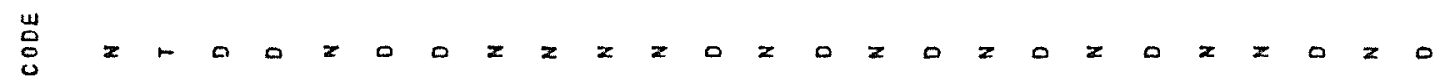

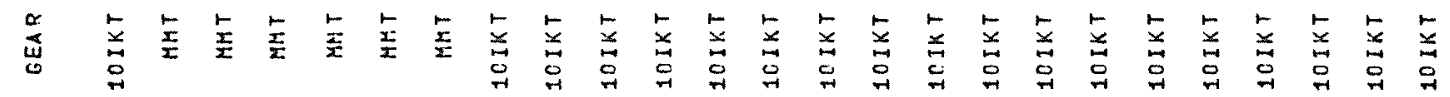

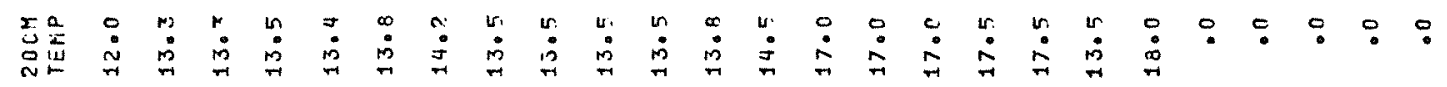
峉是

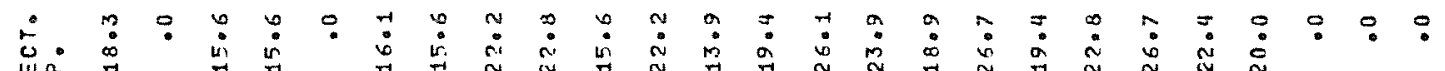

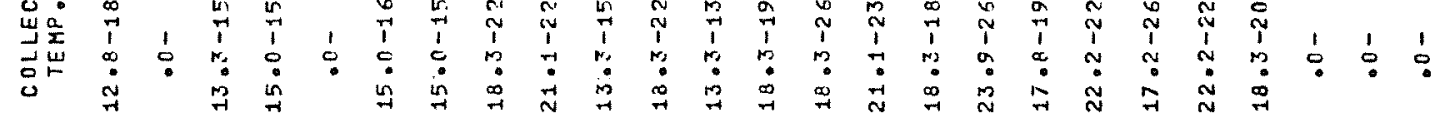

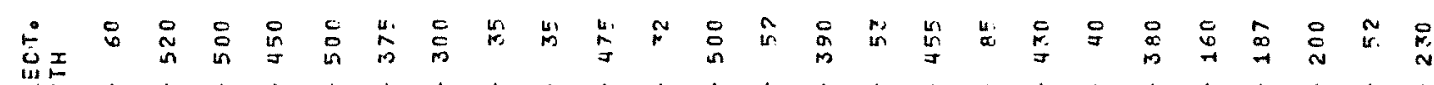

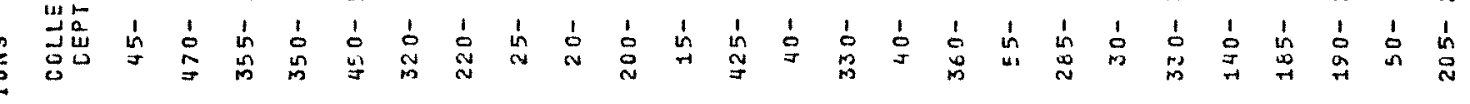

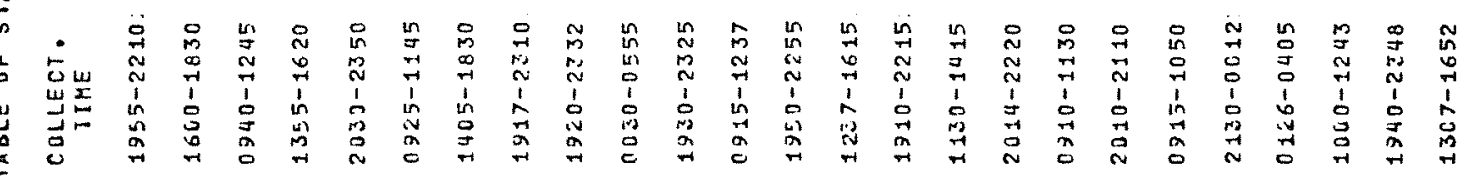

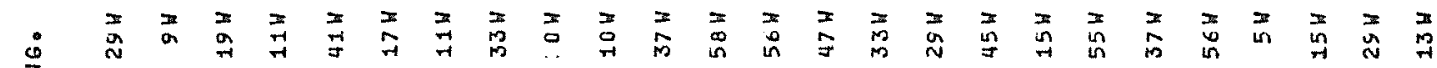

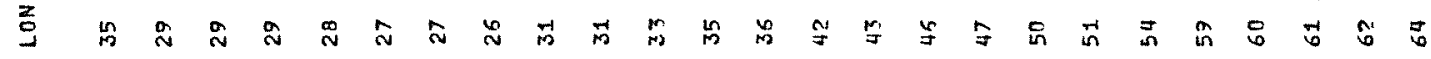

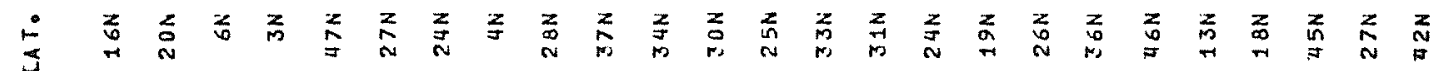

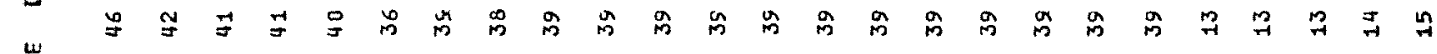

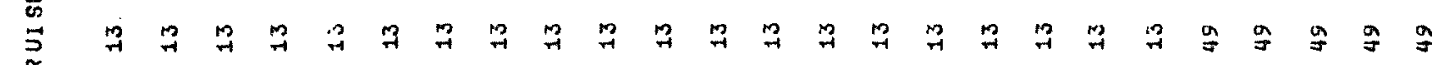

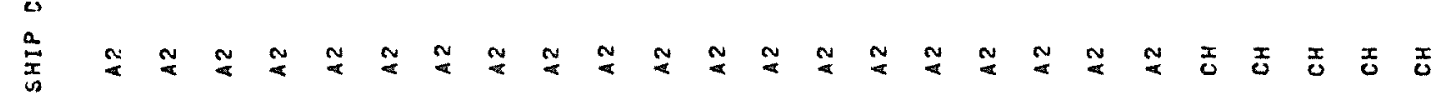

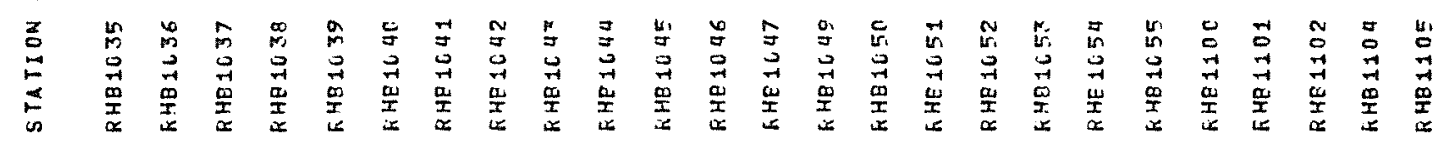




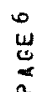

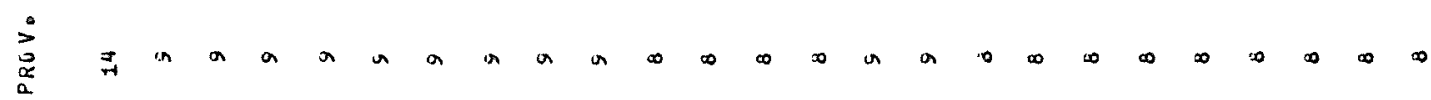

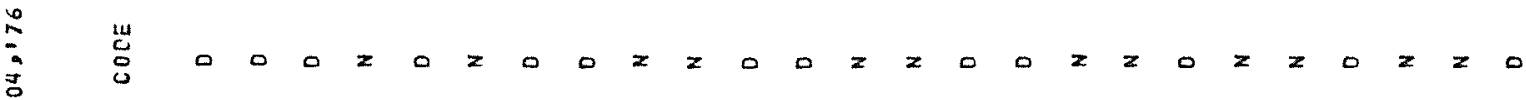

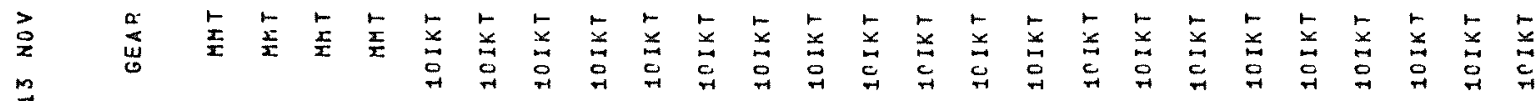

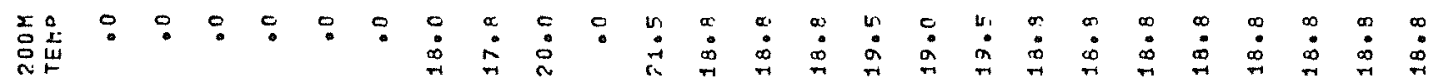

崖竞

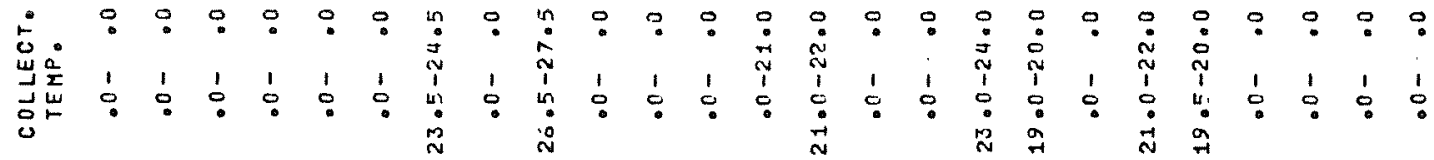

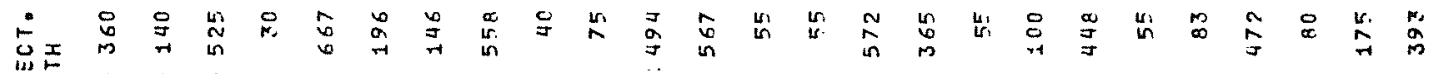

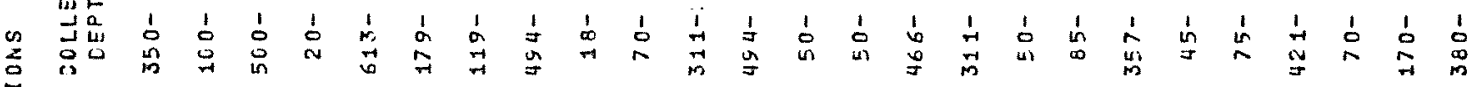

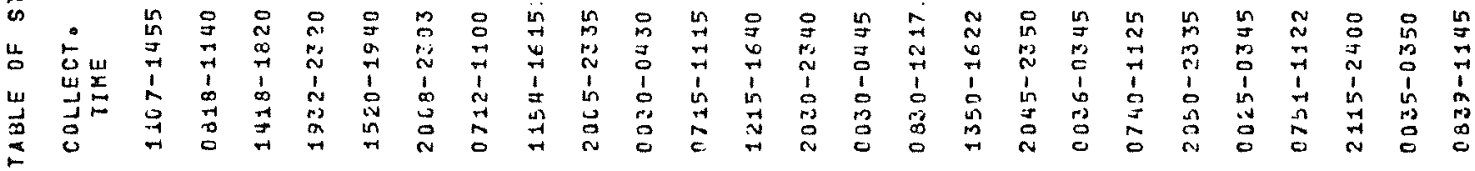

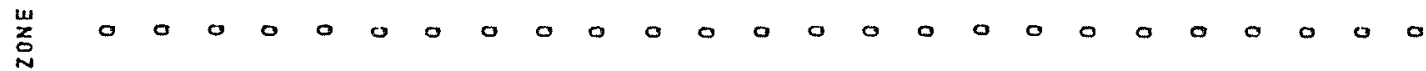

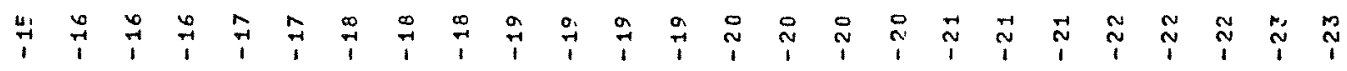

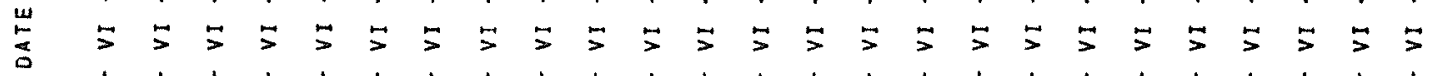

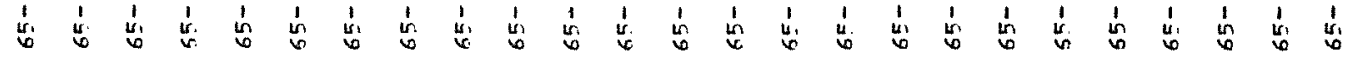

定

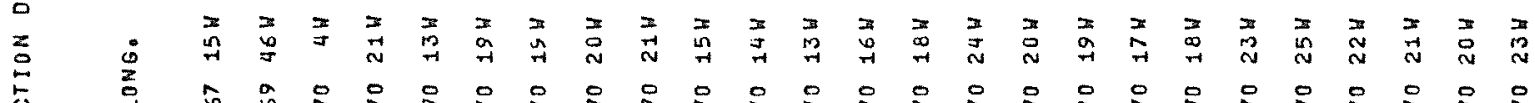

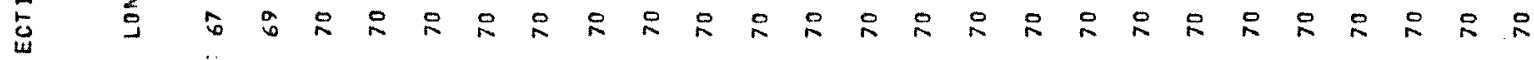

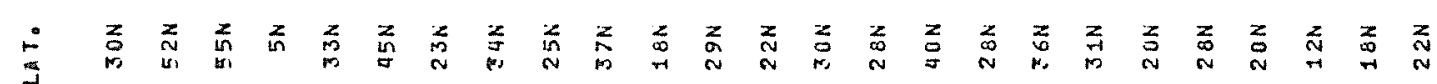

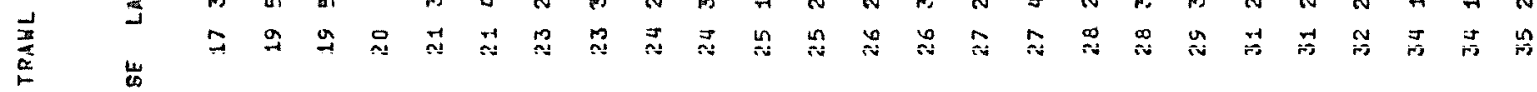

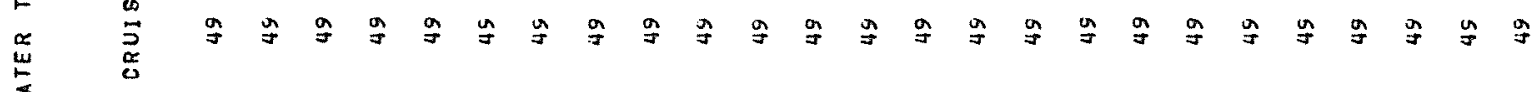

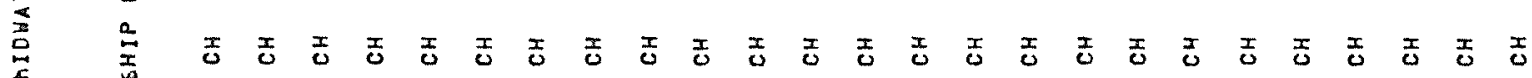

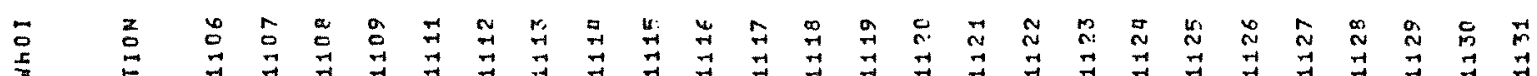

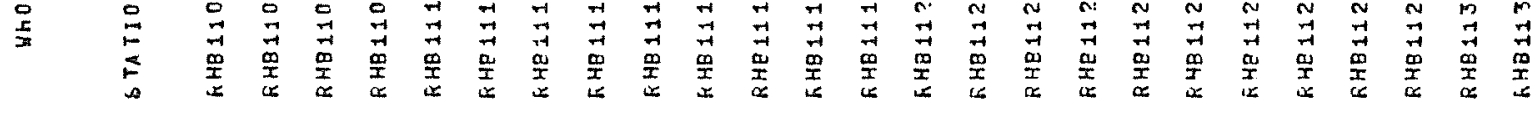




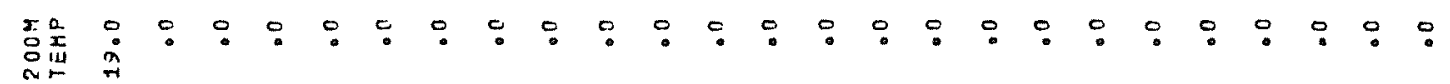

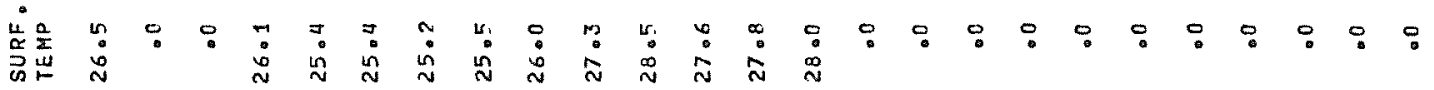

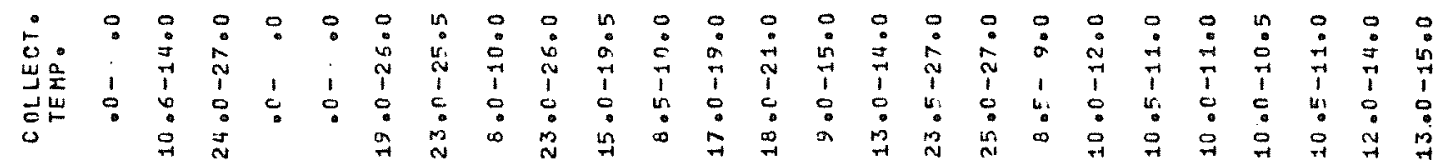

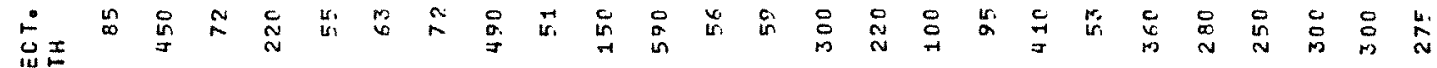

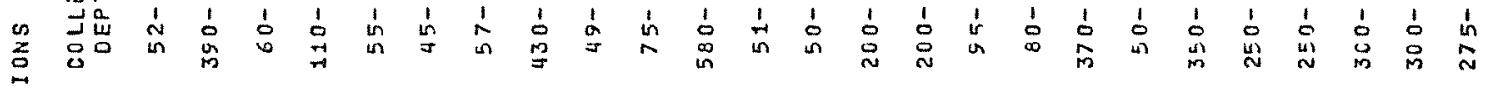
点

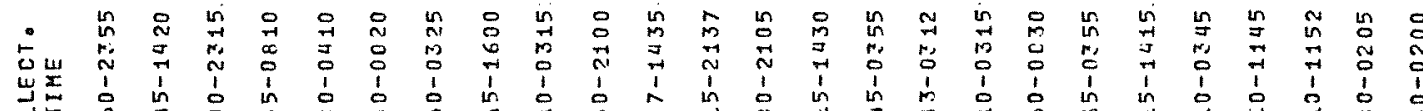

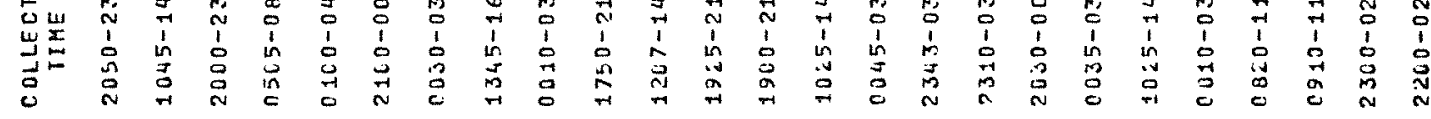

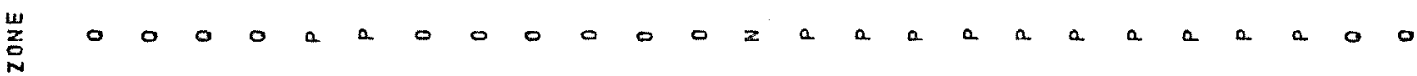

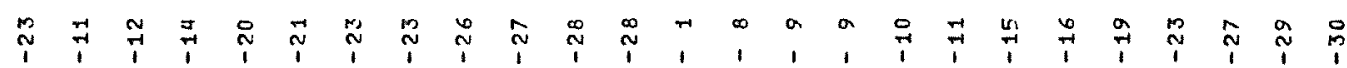

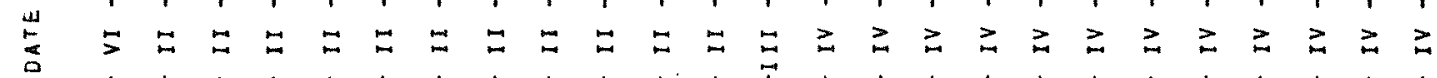

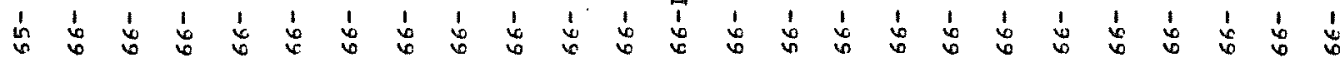




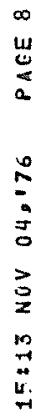

总

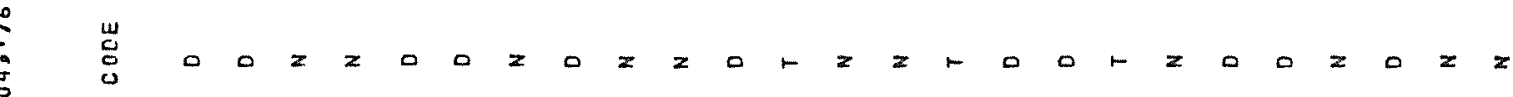

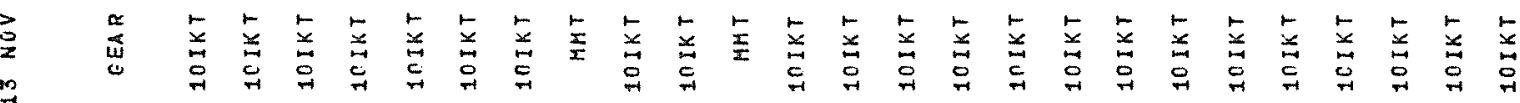

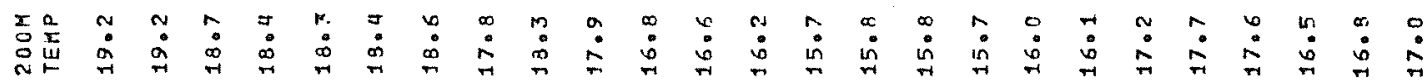
茎足

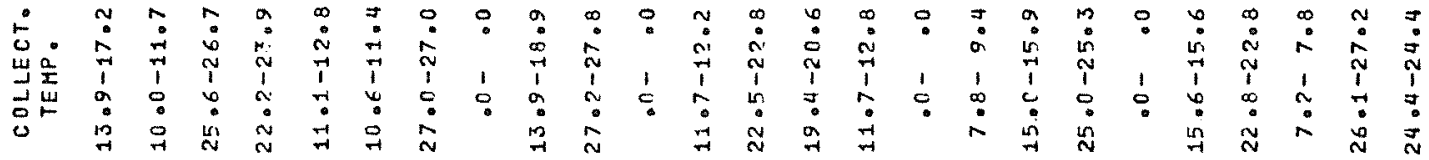

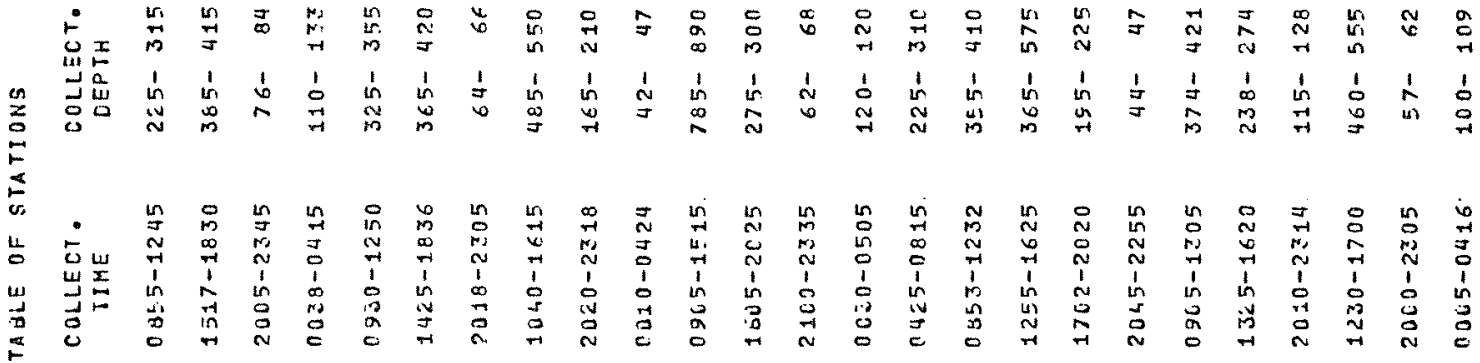

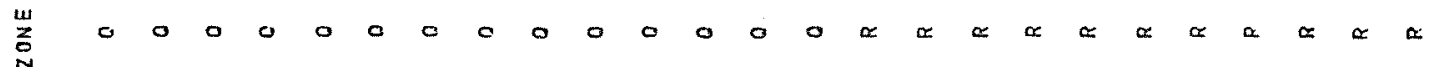

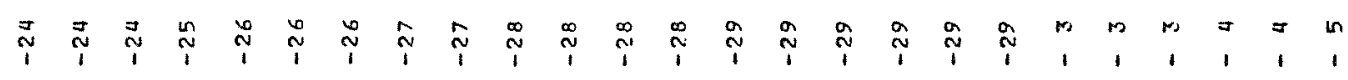

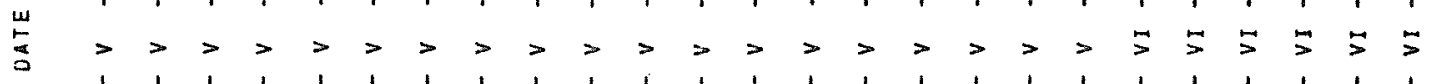

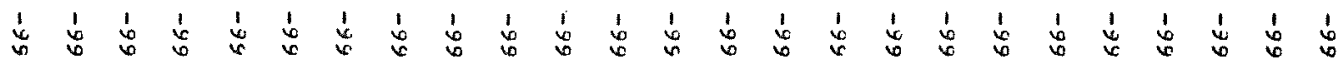

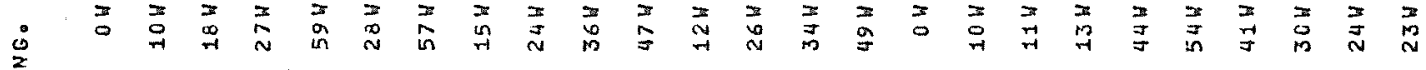

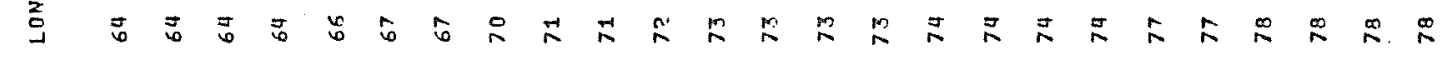

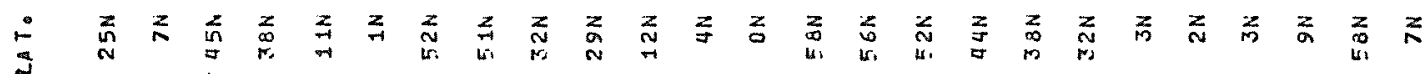
宸

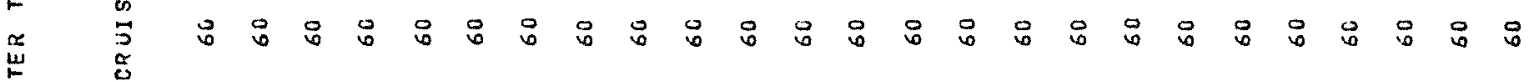

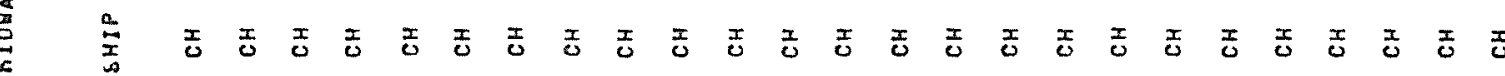

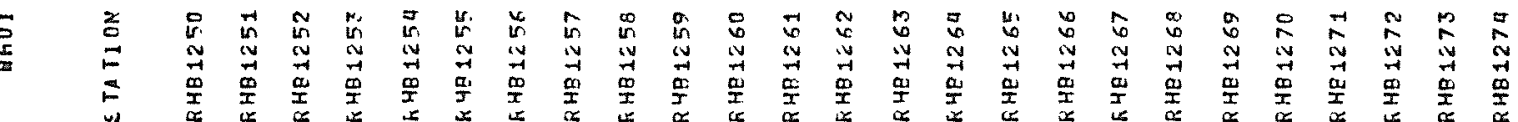




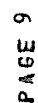

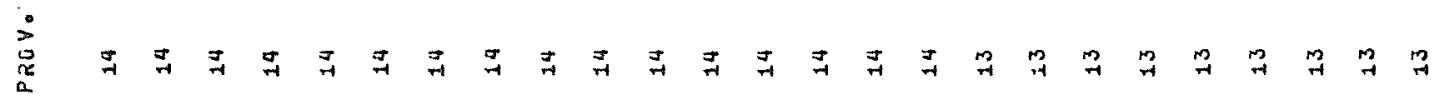

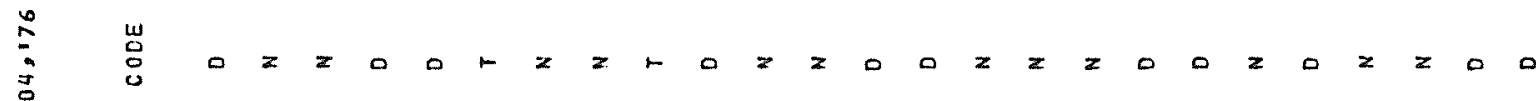

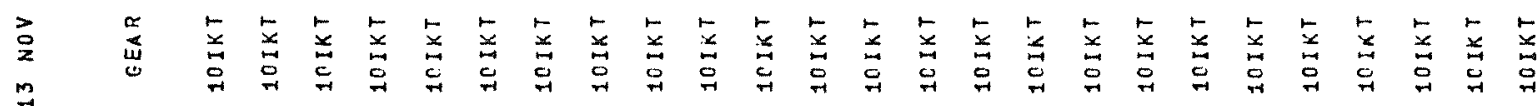

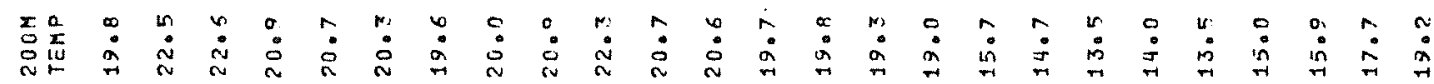

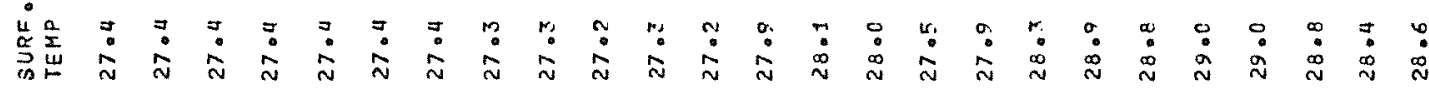

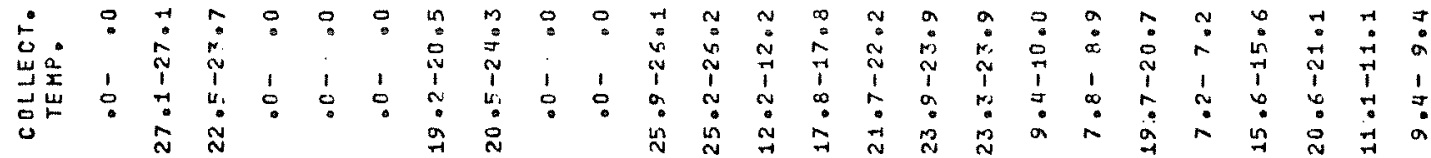

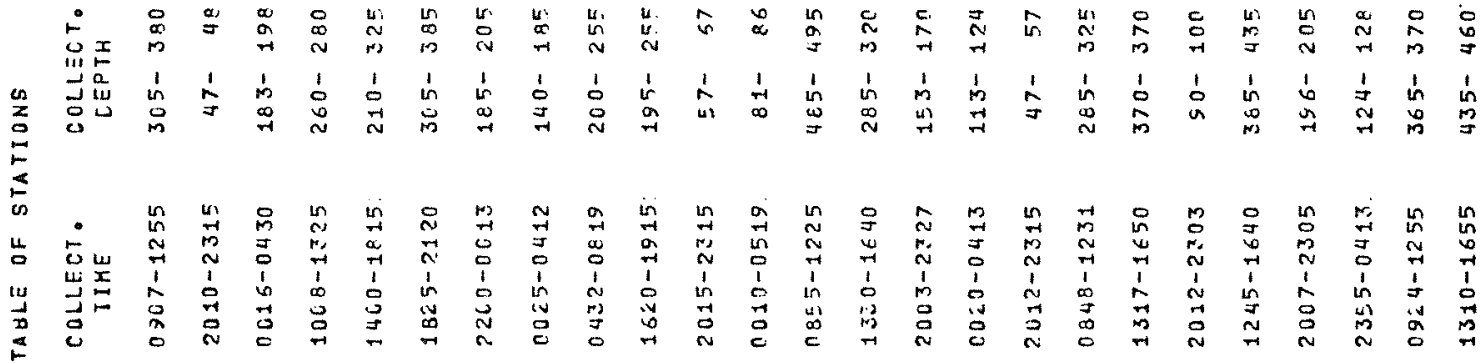

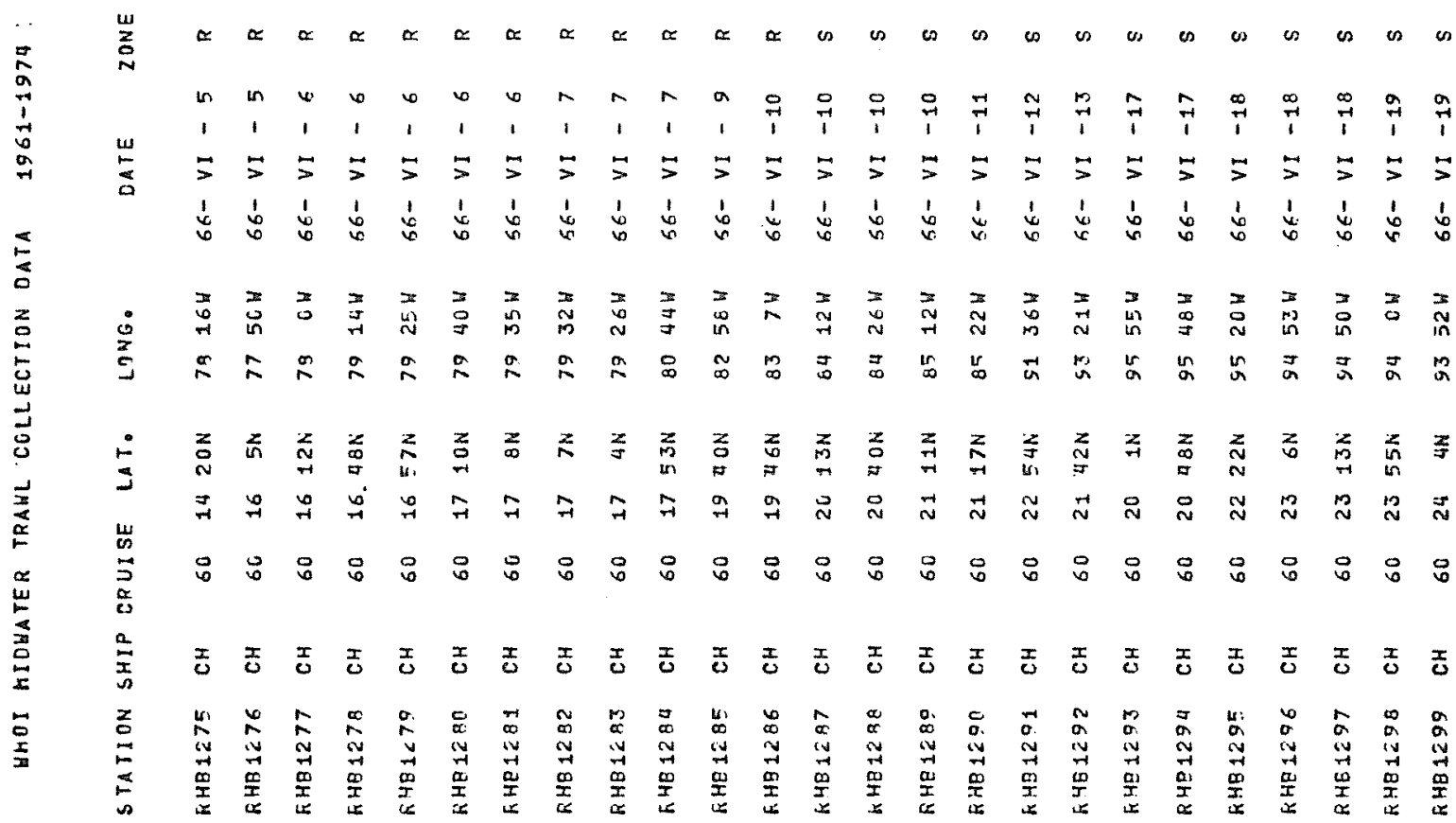


$I-1 I$

우

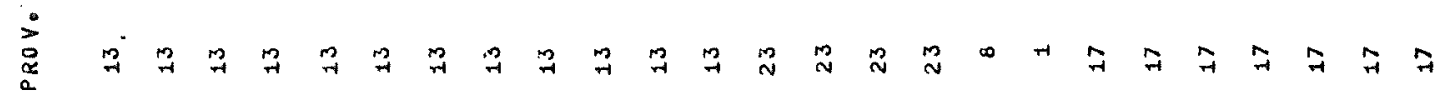
:

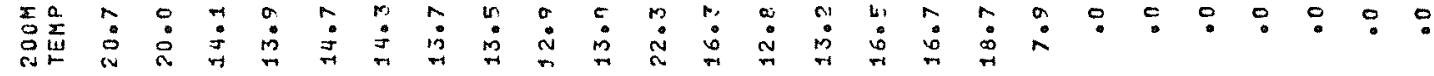

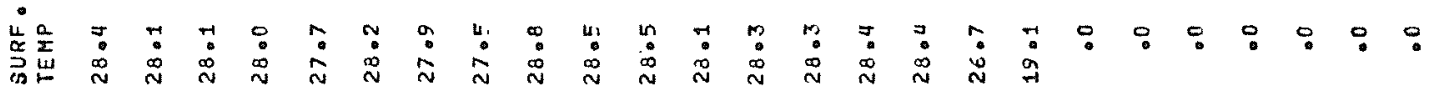

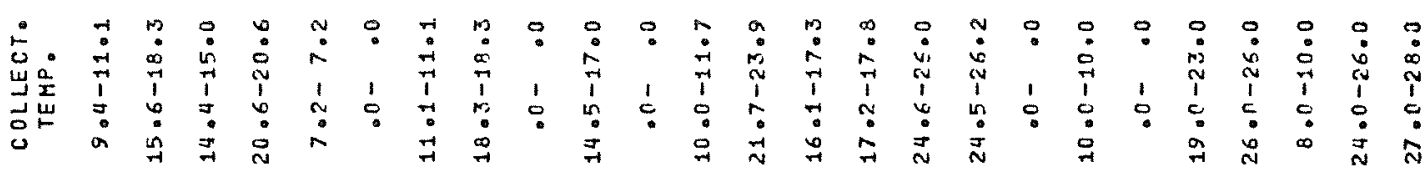

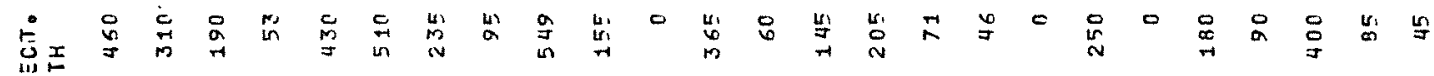
留

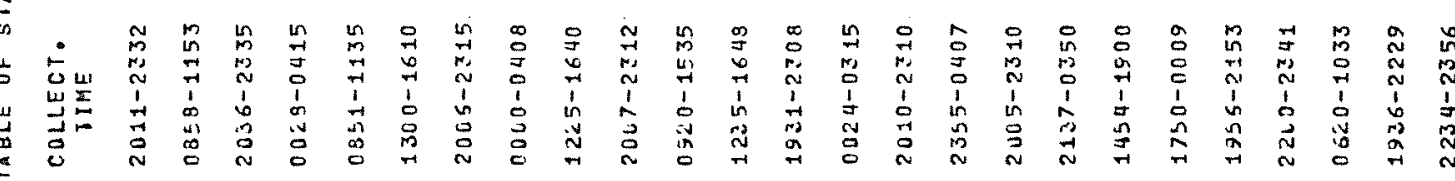

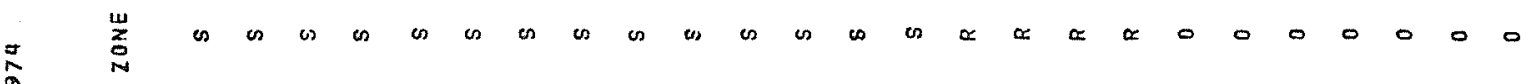

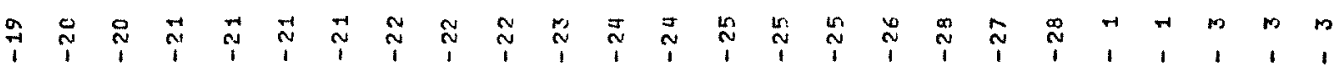

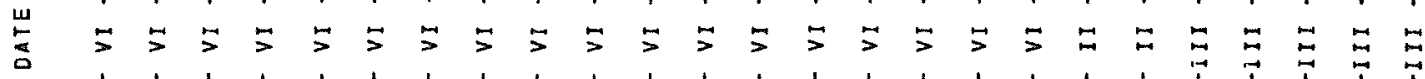

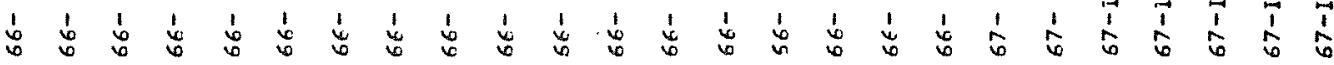

5

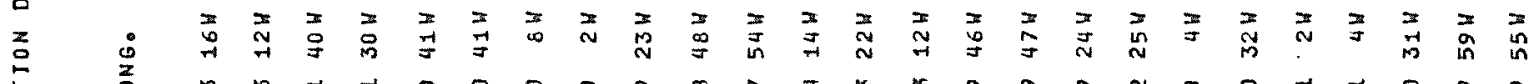

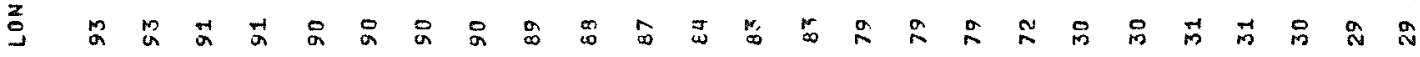

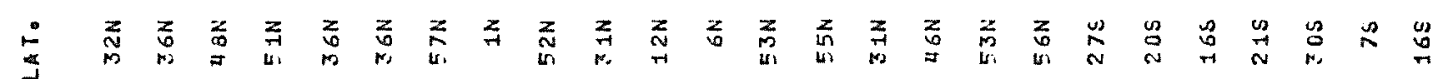
疋 崩 药 I

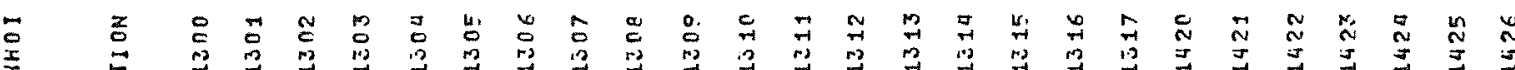

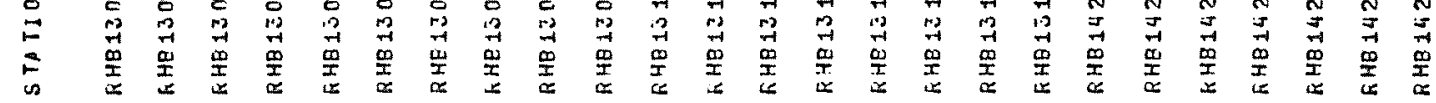




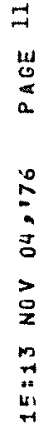

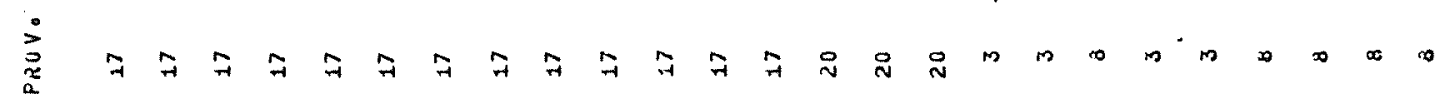

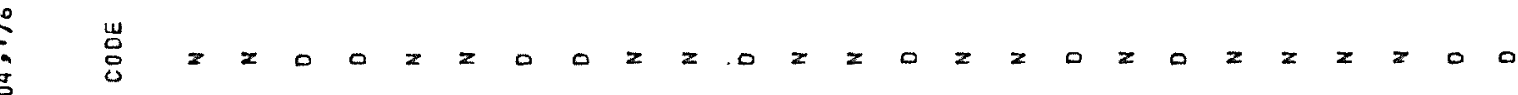

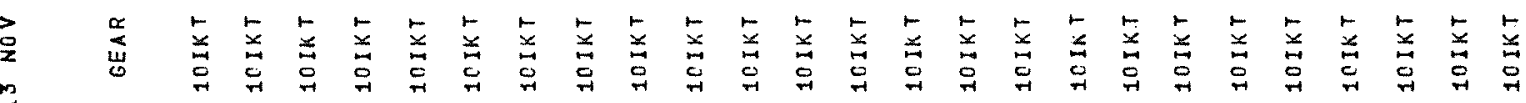

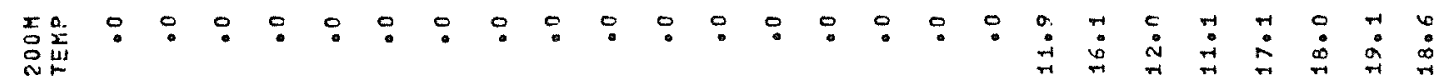

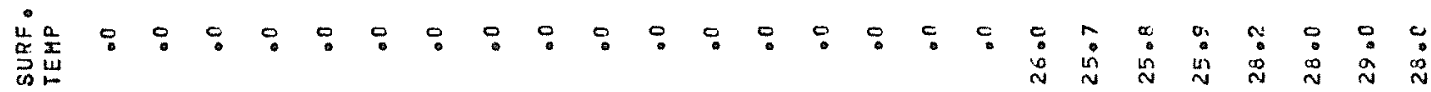

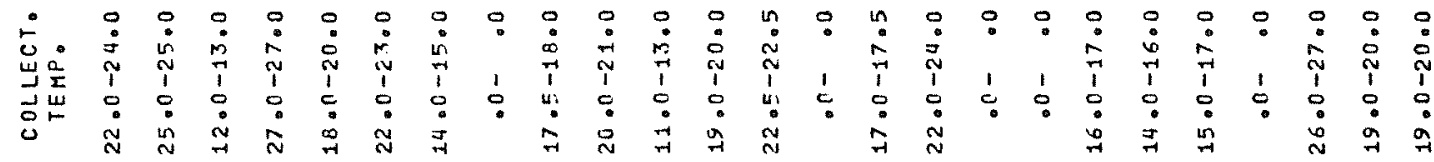

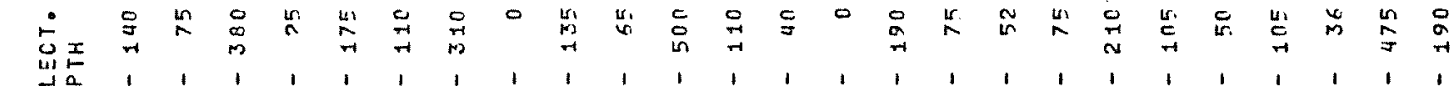

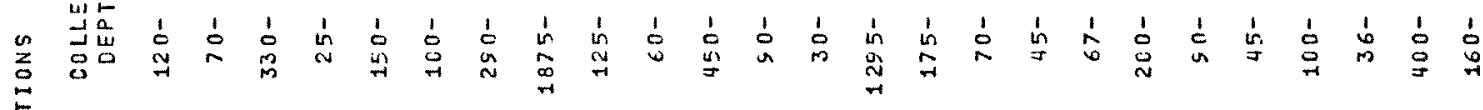
is

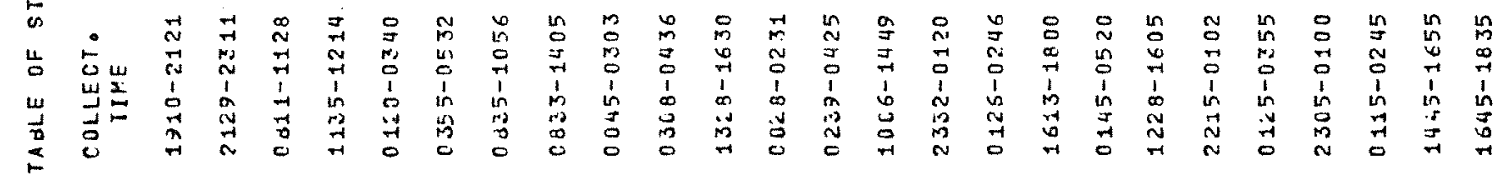

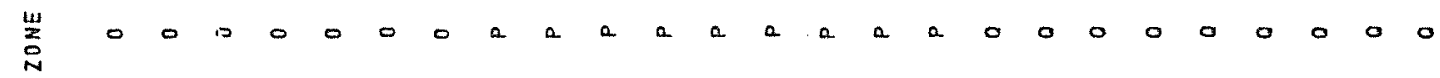

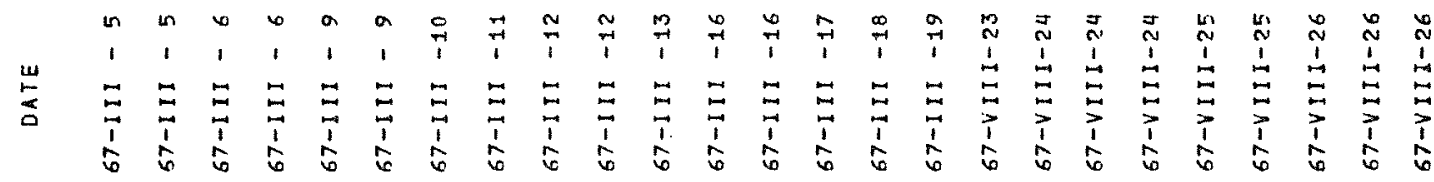
$\$$

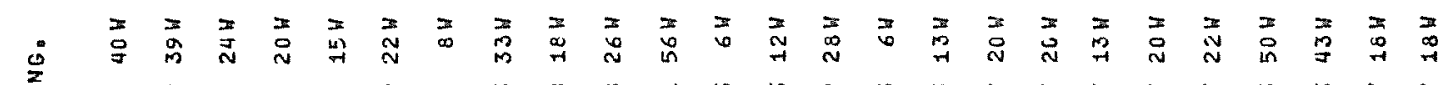

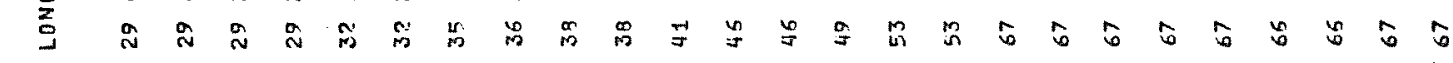

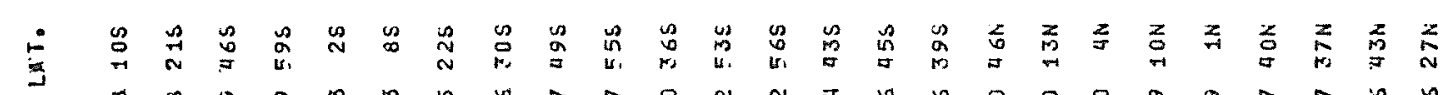

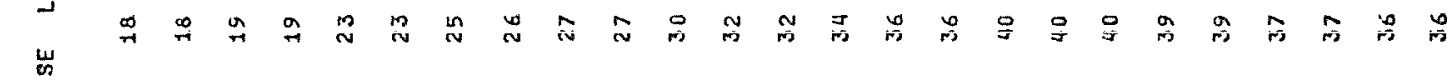

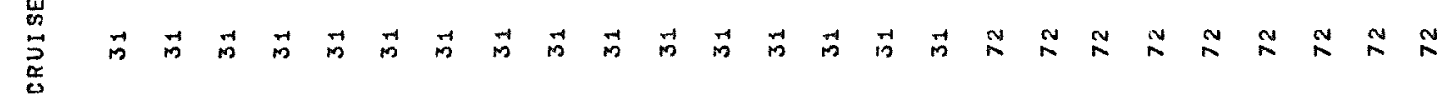

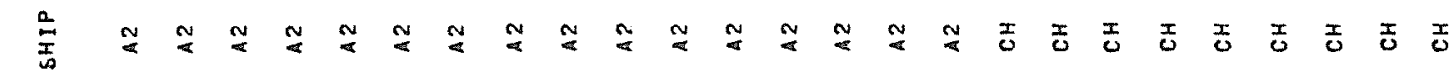

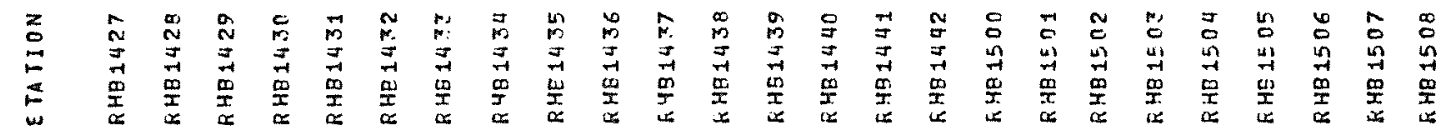




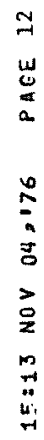

$\dot{\alpha}$
$\frac{\alpha}{\alpha}$ $\frac{0}{5}$ :

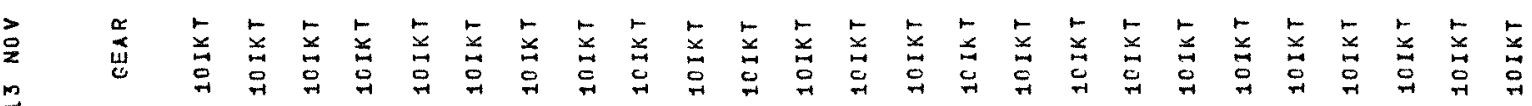

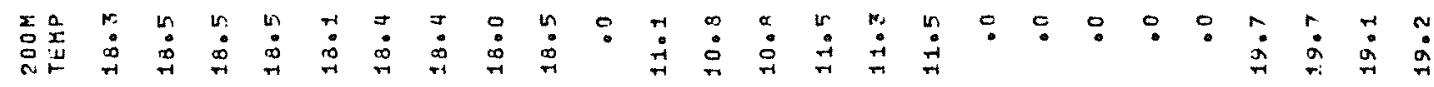

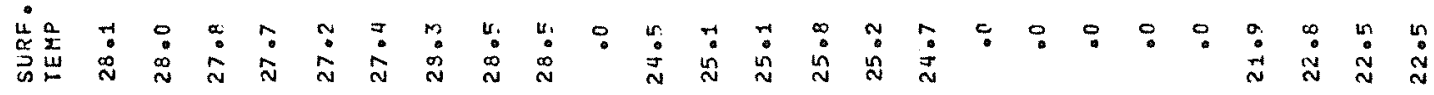

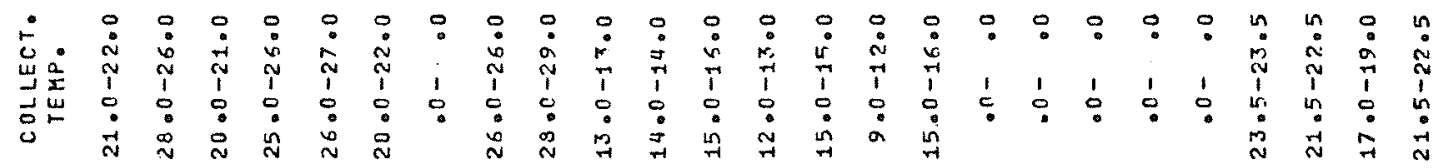

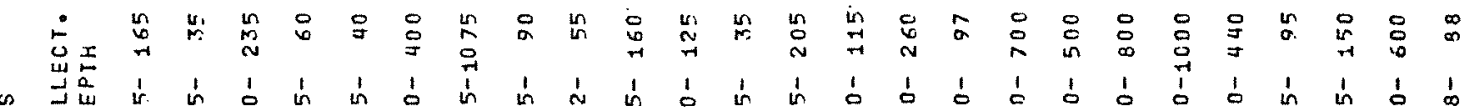

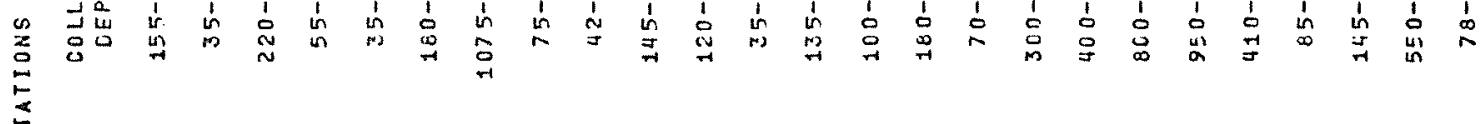

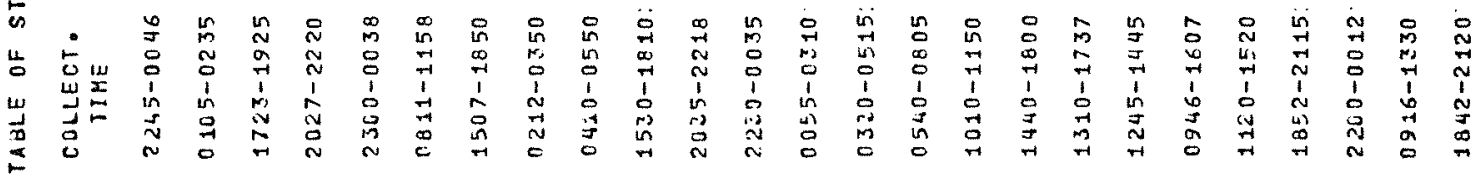

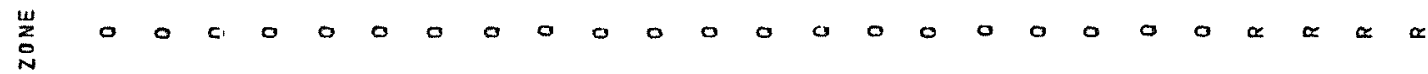

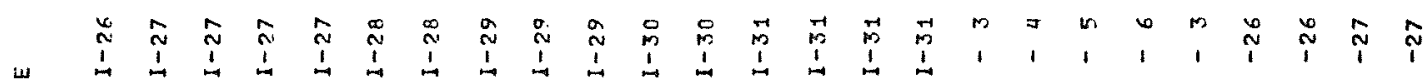

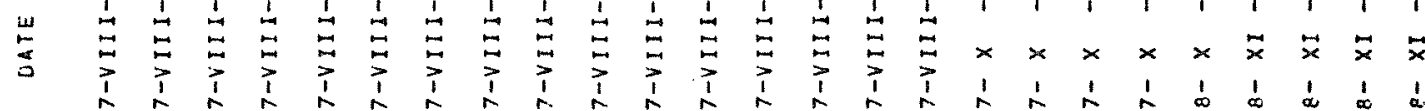


3
4
0
0
0
0
0
5
0
0
0
2
0
0

$\dot{0}$
$\dot{0}$
0 :

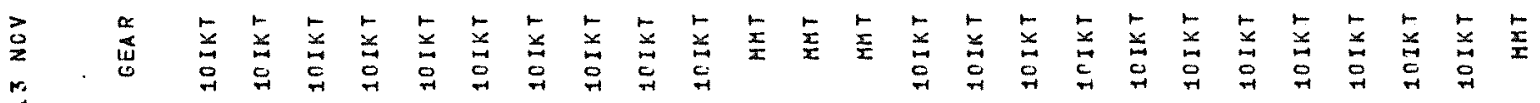

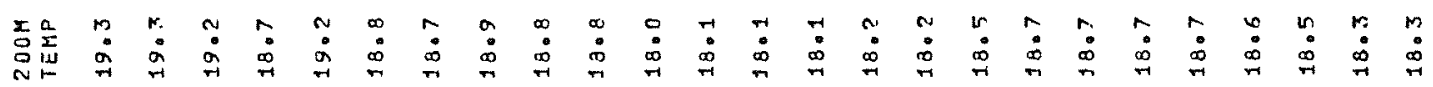

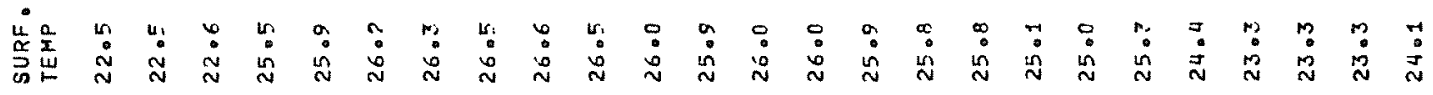

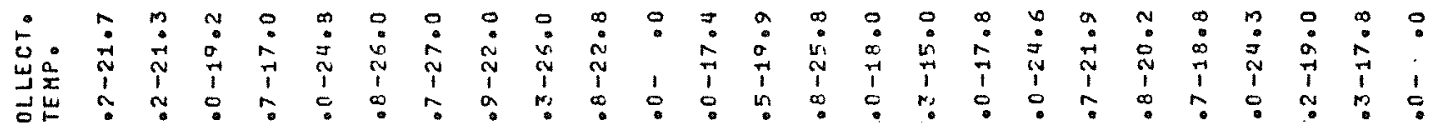

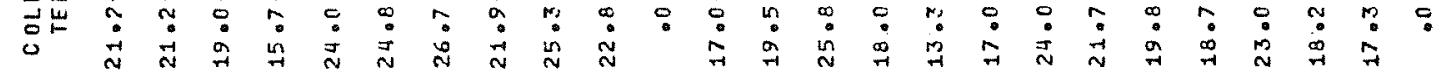

递垔

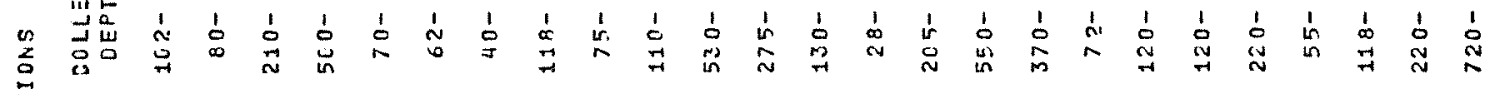
点

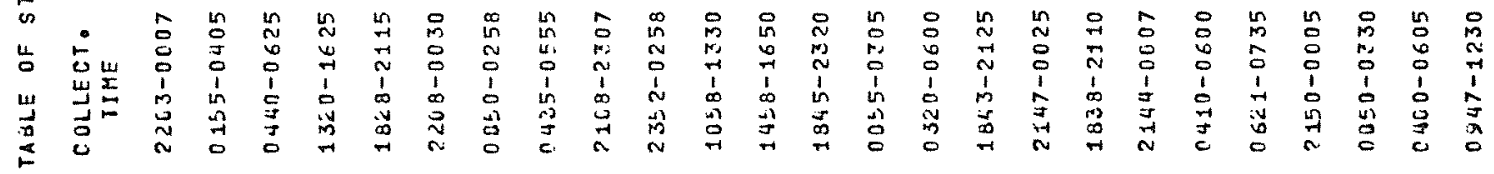

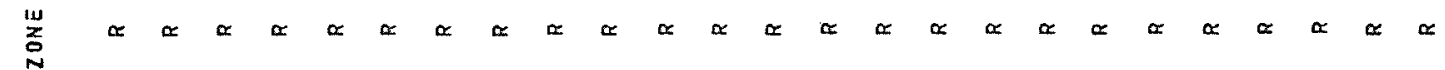

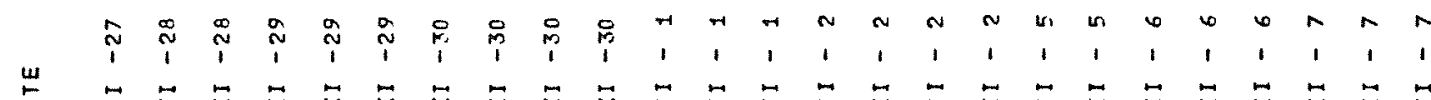

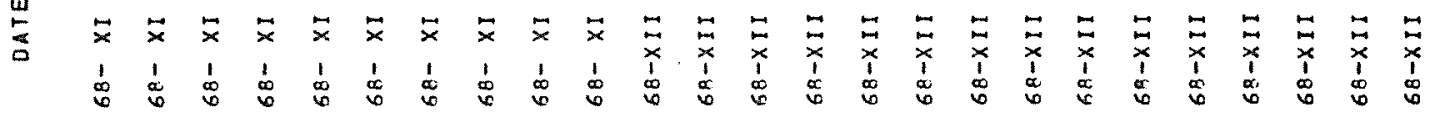

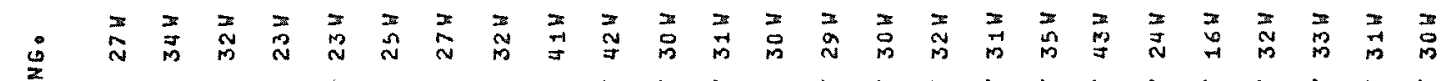

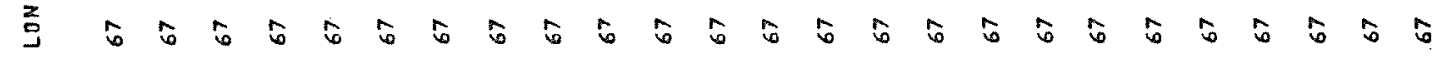

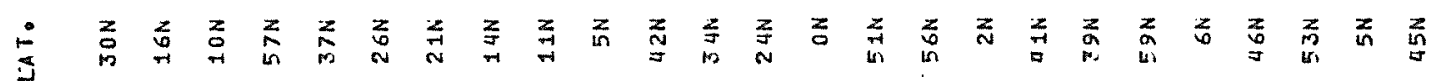

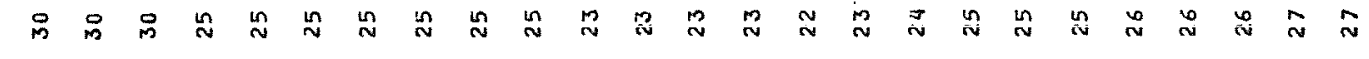

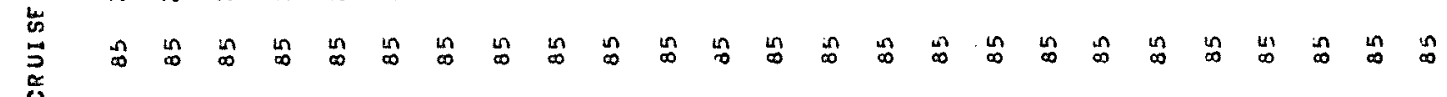
产 J

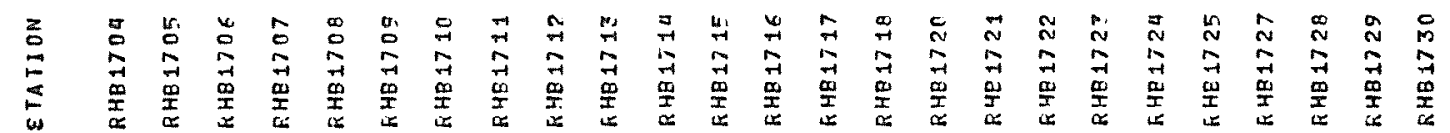




$$
\underset{\grave{a}}{\grave{a}}
$$
0
0
0
2
2
0
$i$
4
4

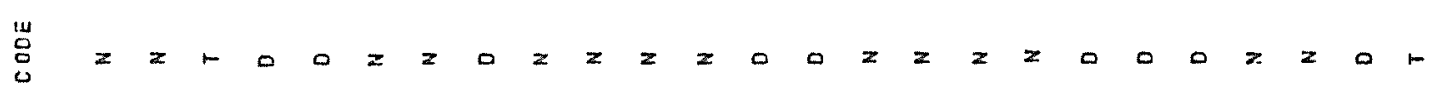

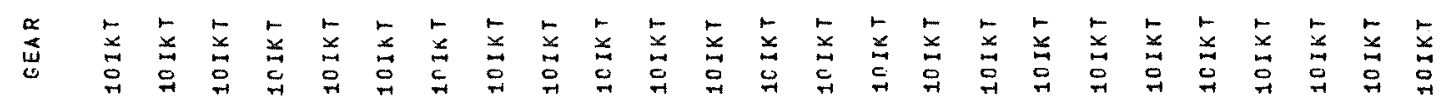

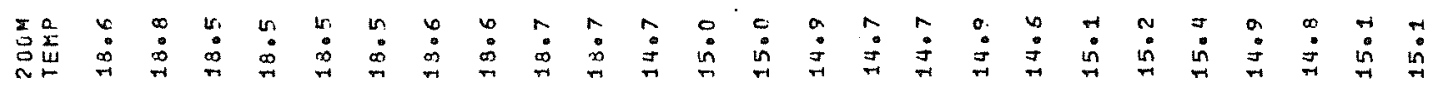

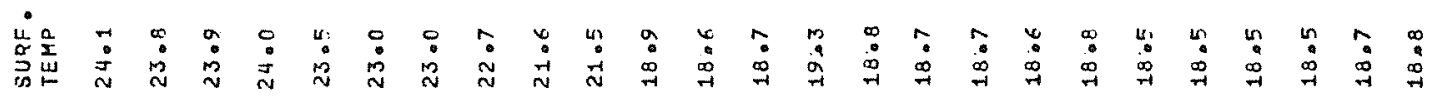

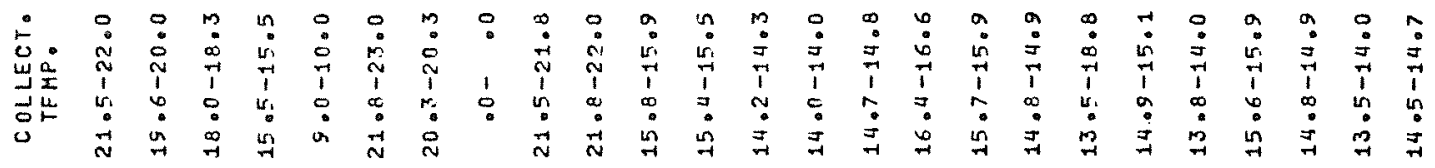

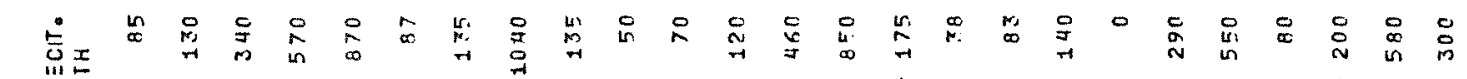

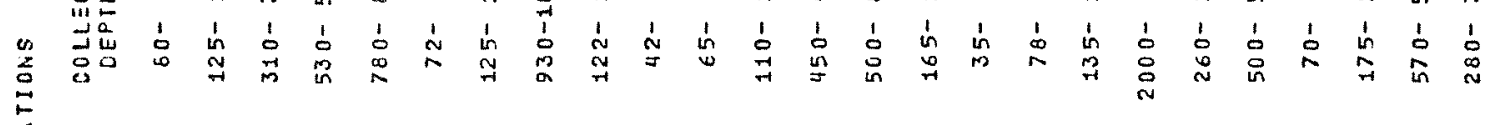

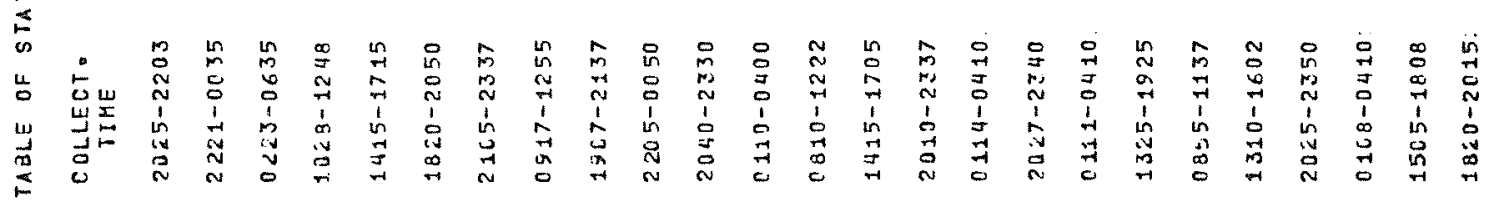
in

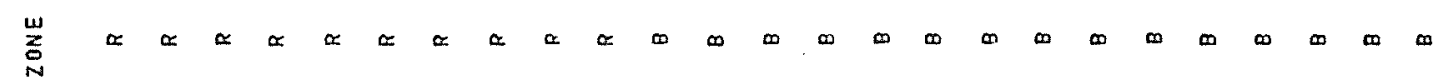

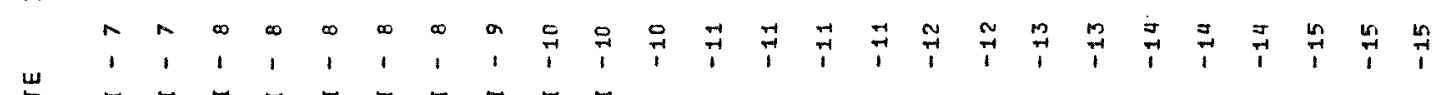

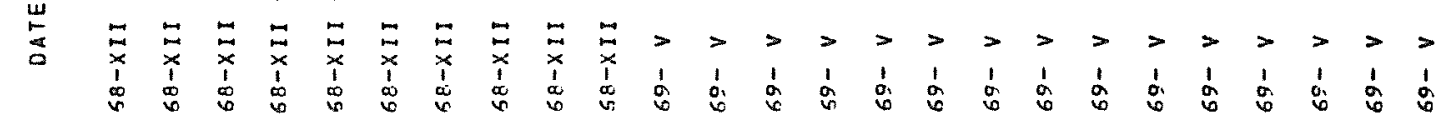

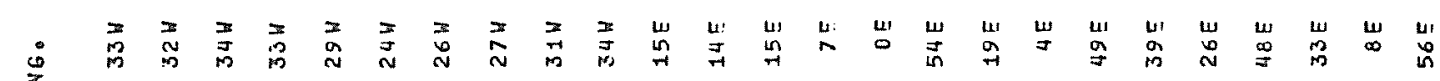
范

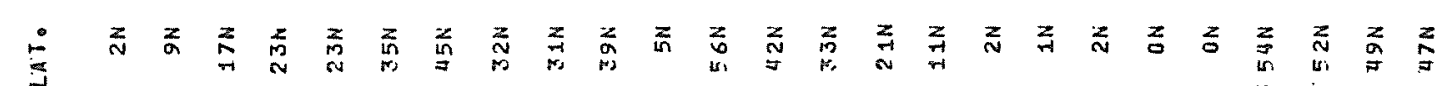

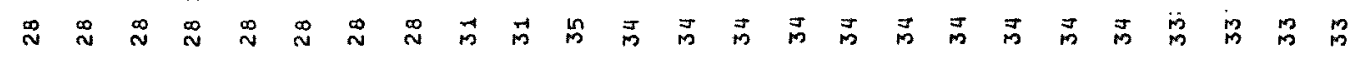
㟶

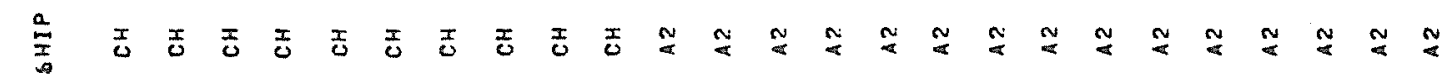

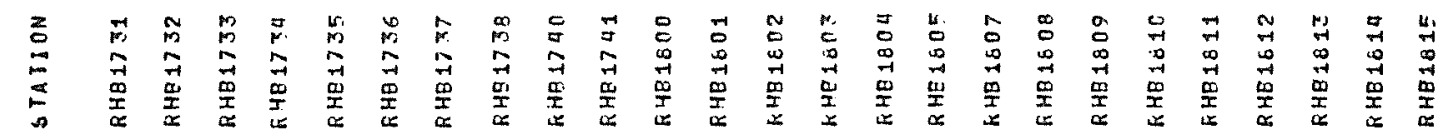


$\cong$

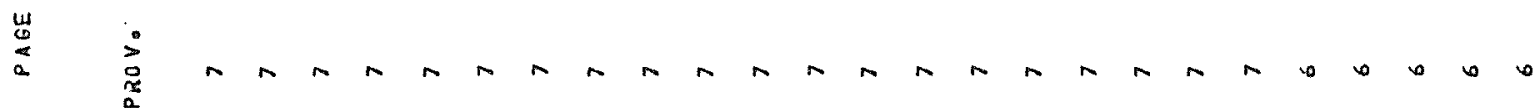

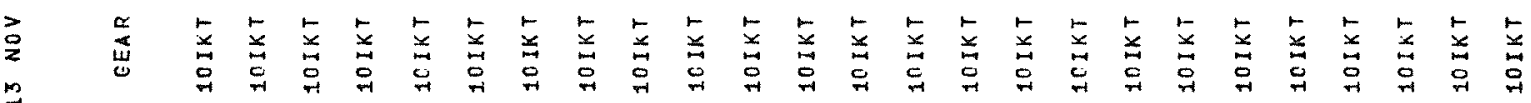

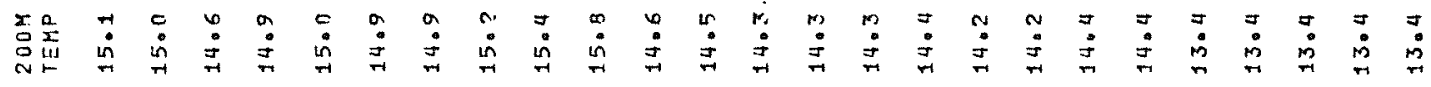

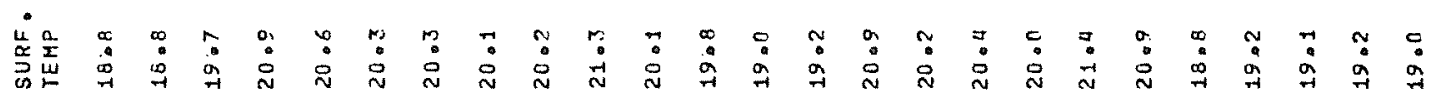

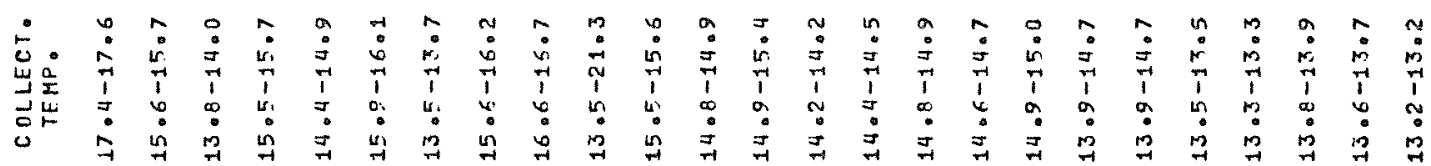

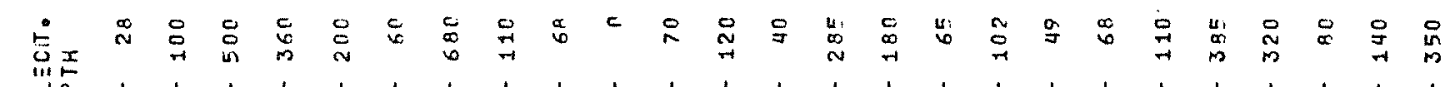

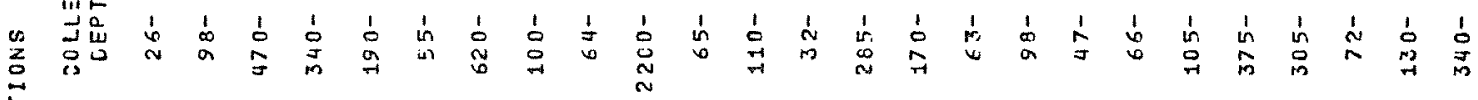

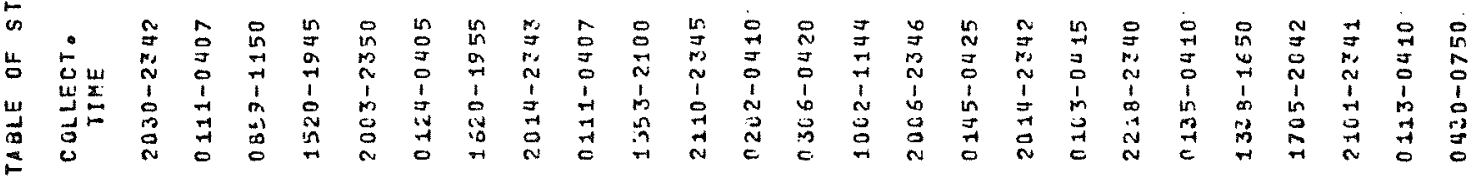

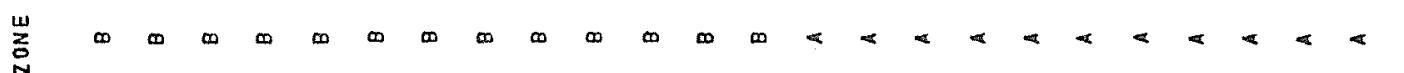
0
$i$
$\vdots$
$i$
0

$\$$

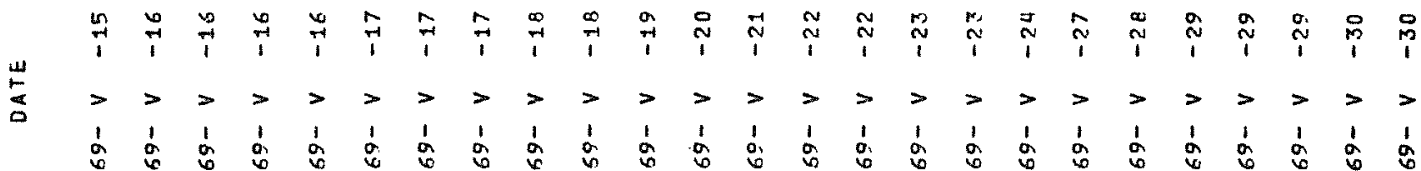

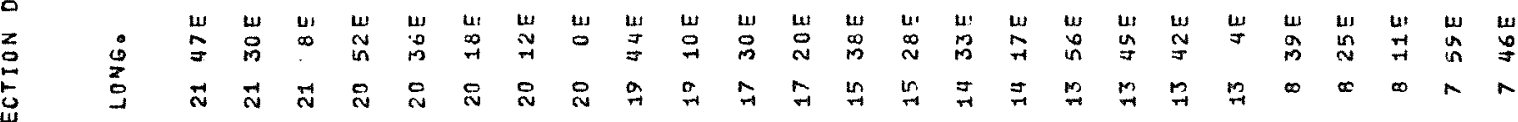

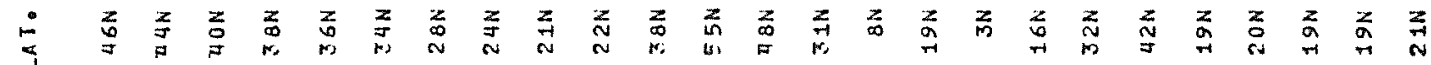

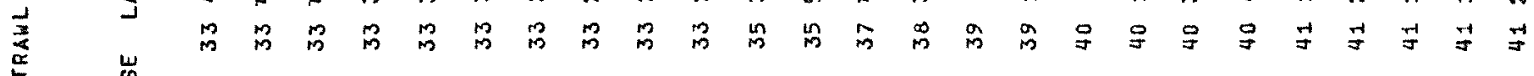

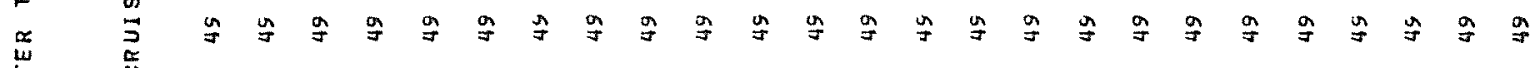

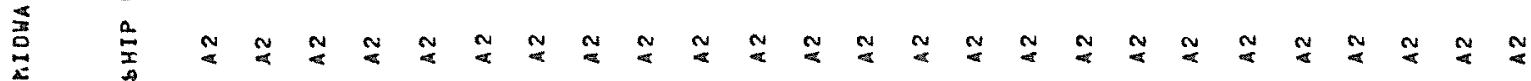

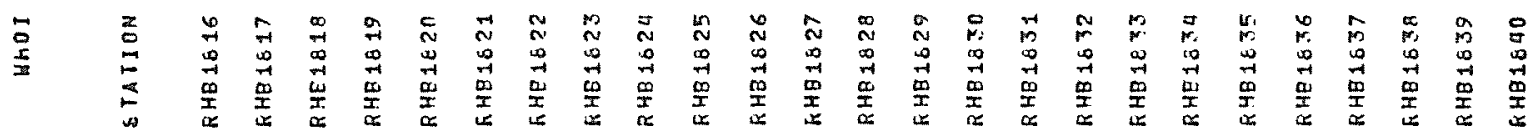


$\stackrel{\infty}{-1}$

$\stackrel{u}{a}$

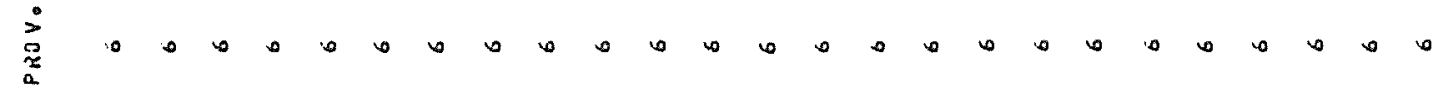

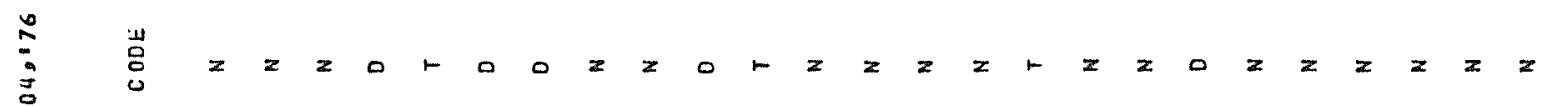

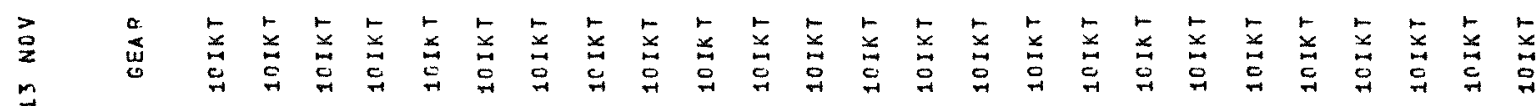
$\underset{\sim}{\ddot{H}}$

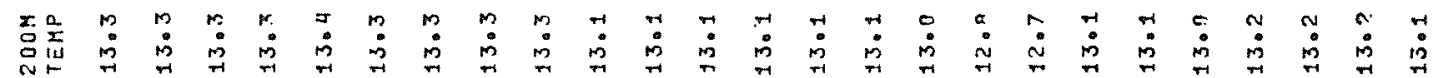

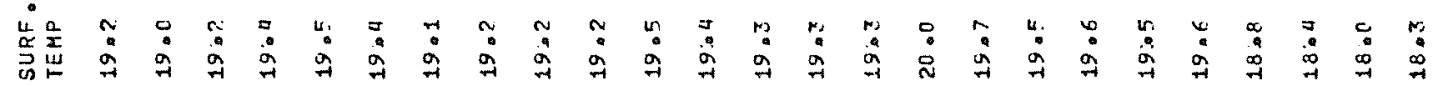

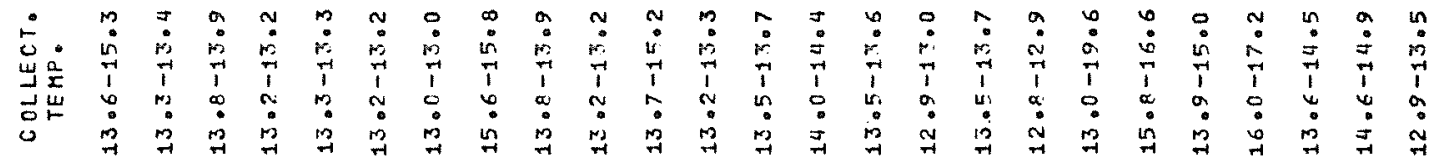

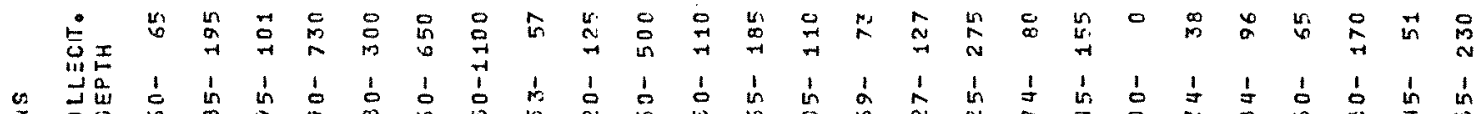

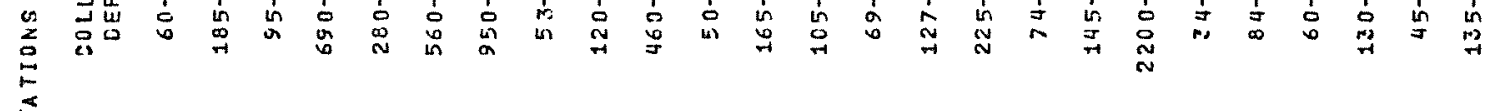

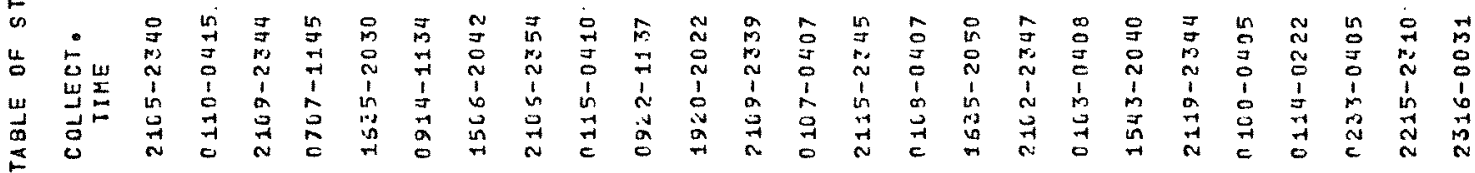

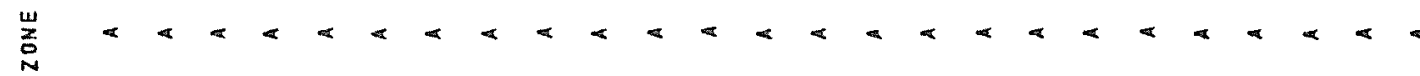

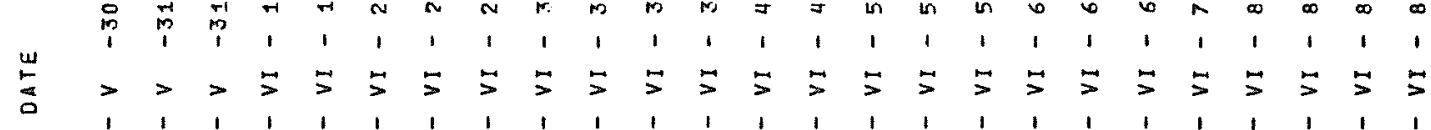
$\$$

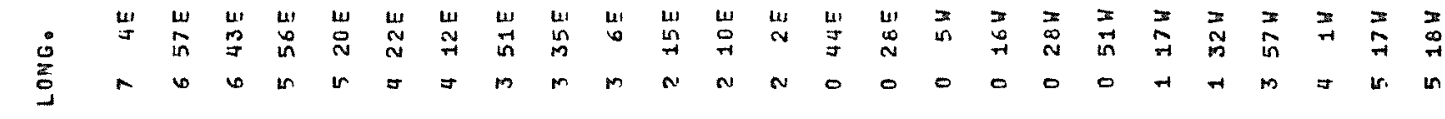

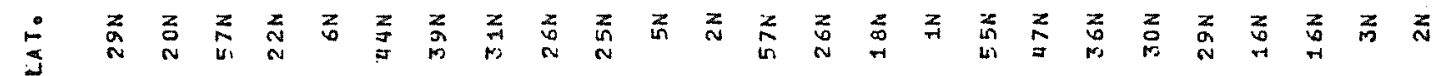

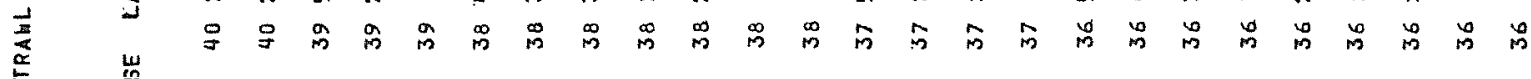
采

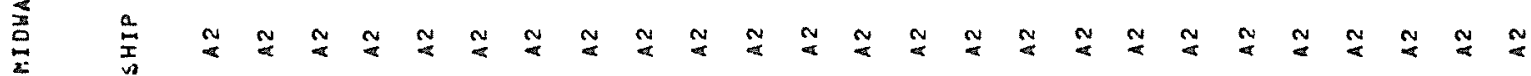

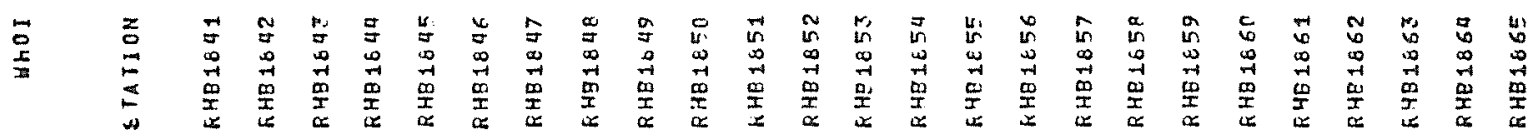


5
0
0
3
0
0

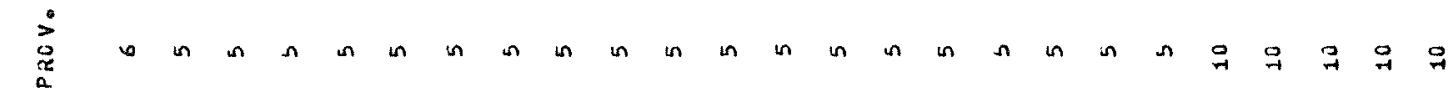
$\vdots$ 宸

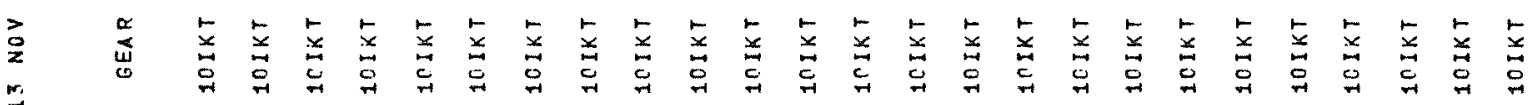

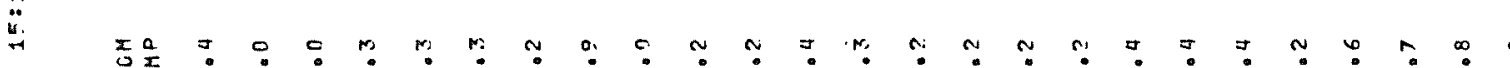

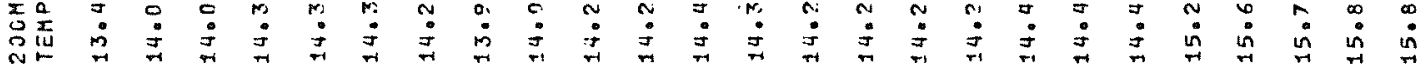

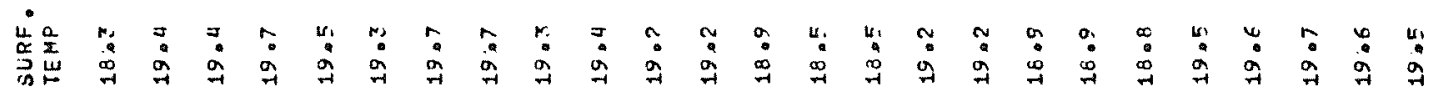

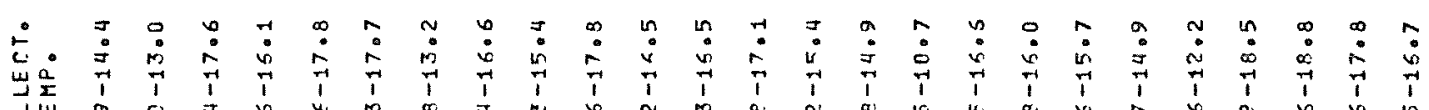

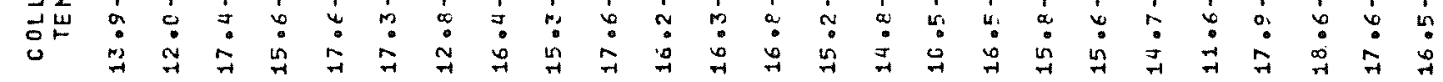

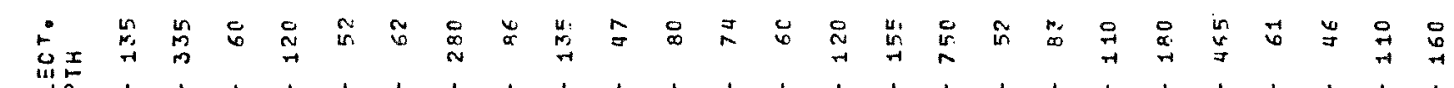

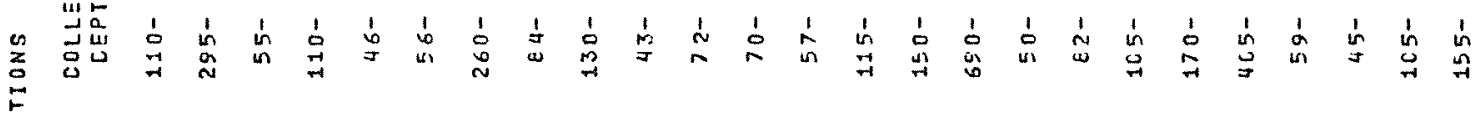

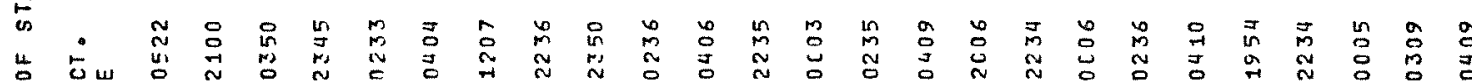

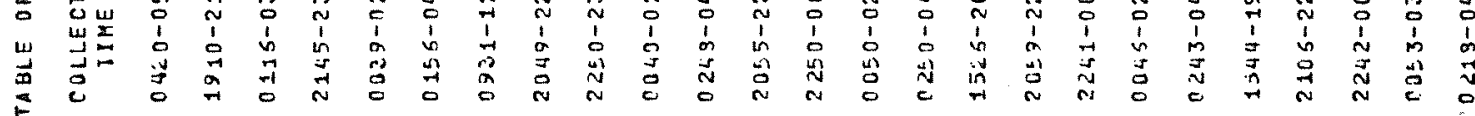

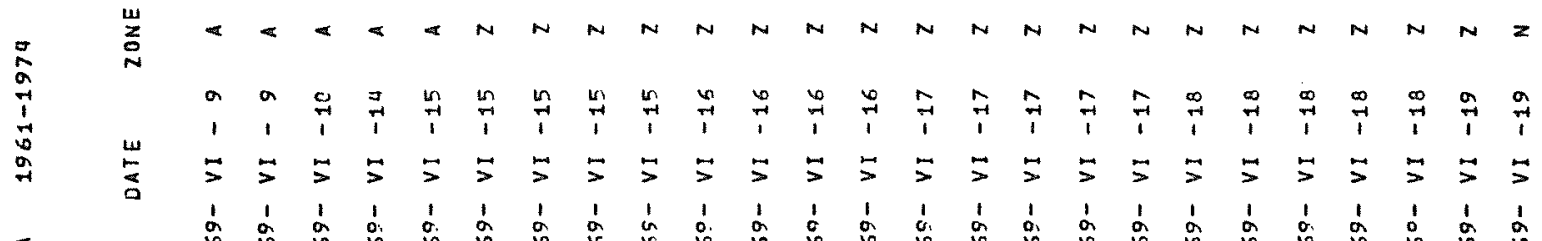

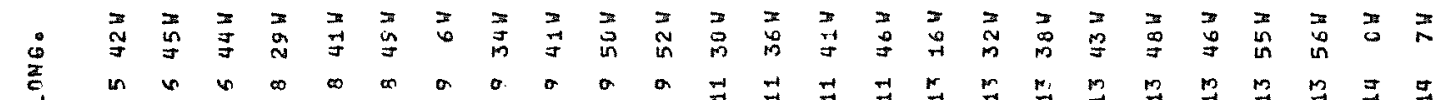

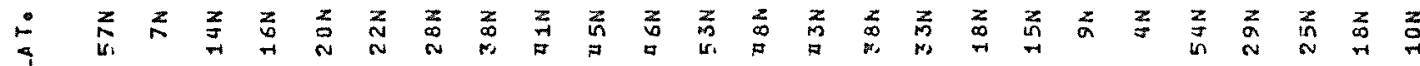

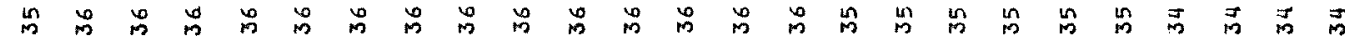

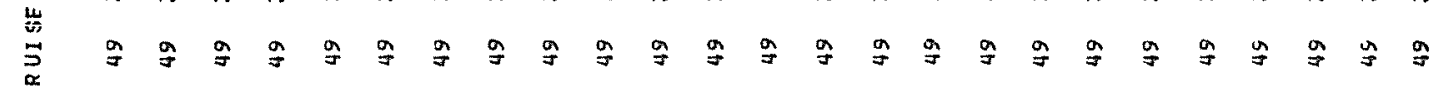

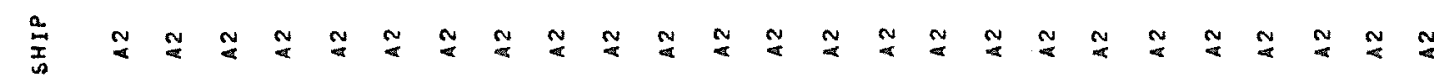

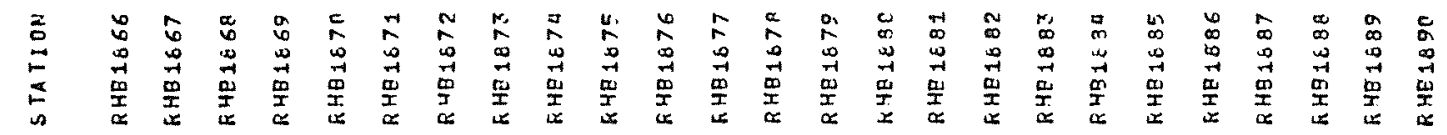


$\infty$

岕

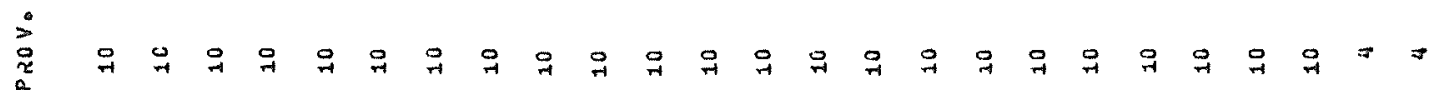

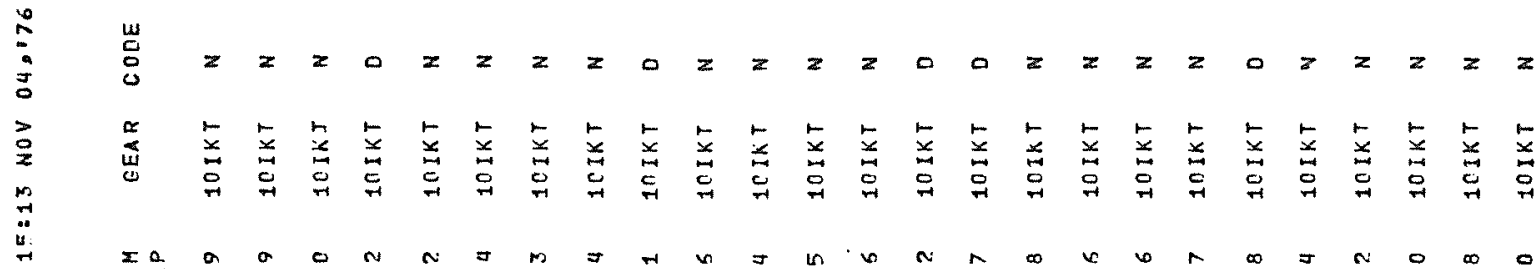

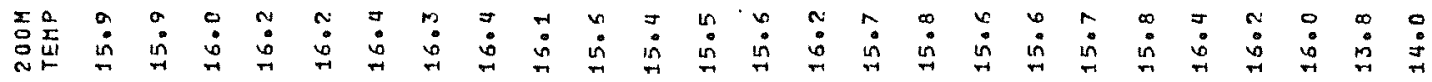

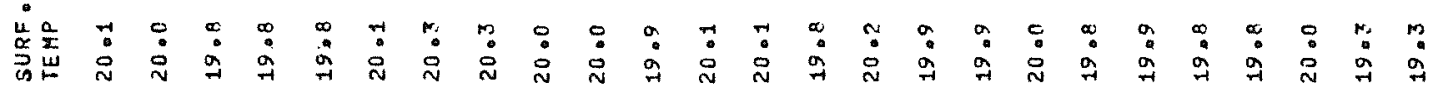

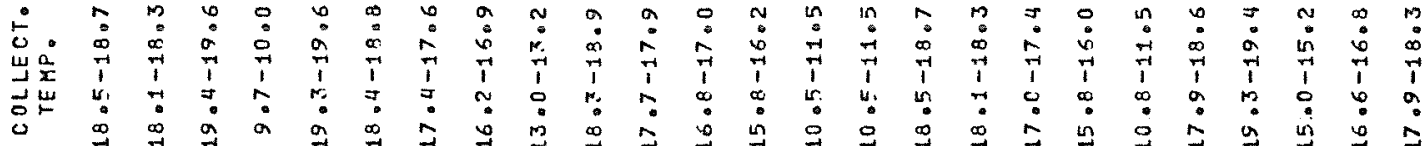

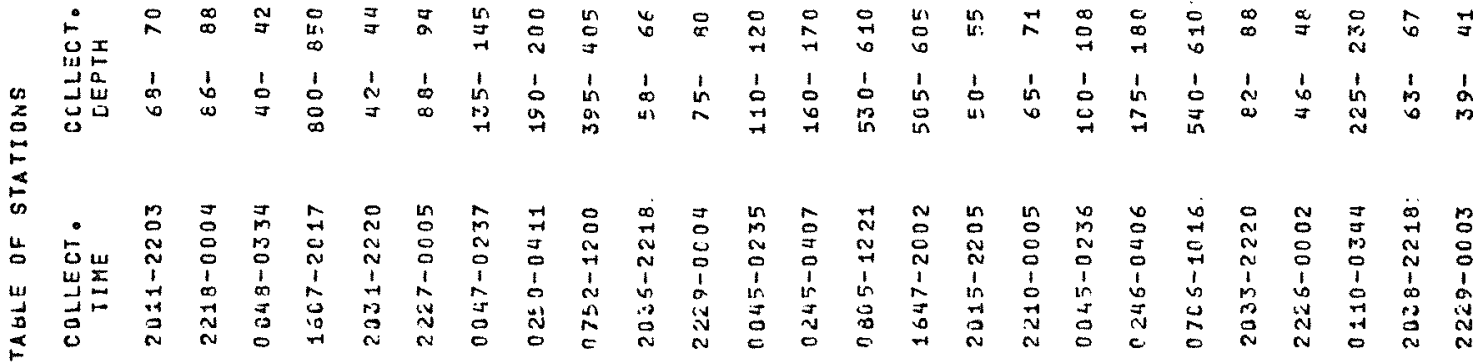

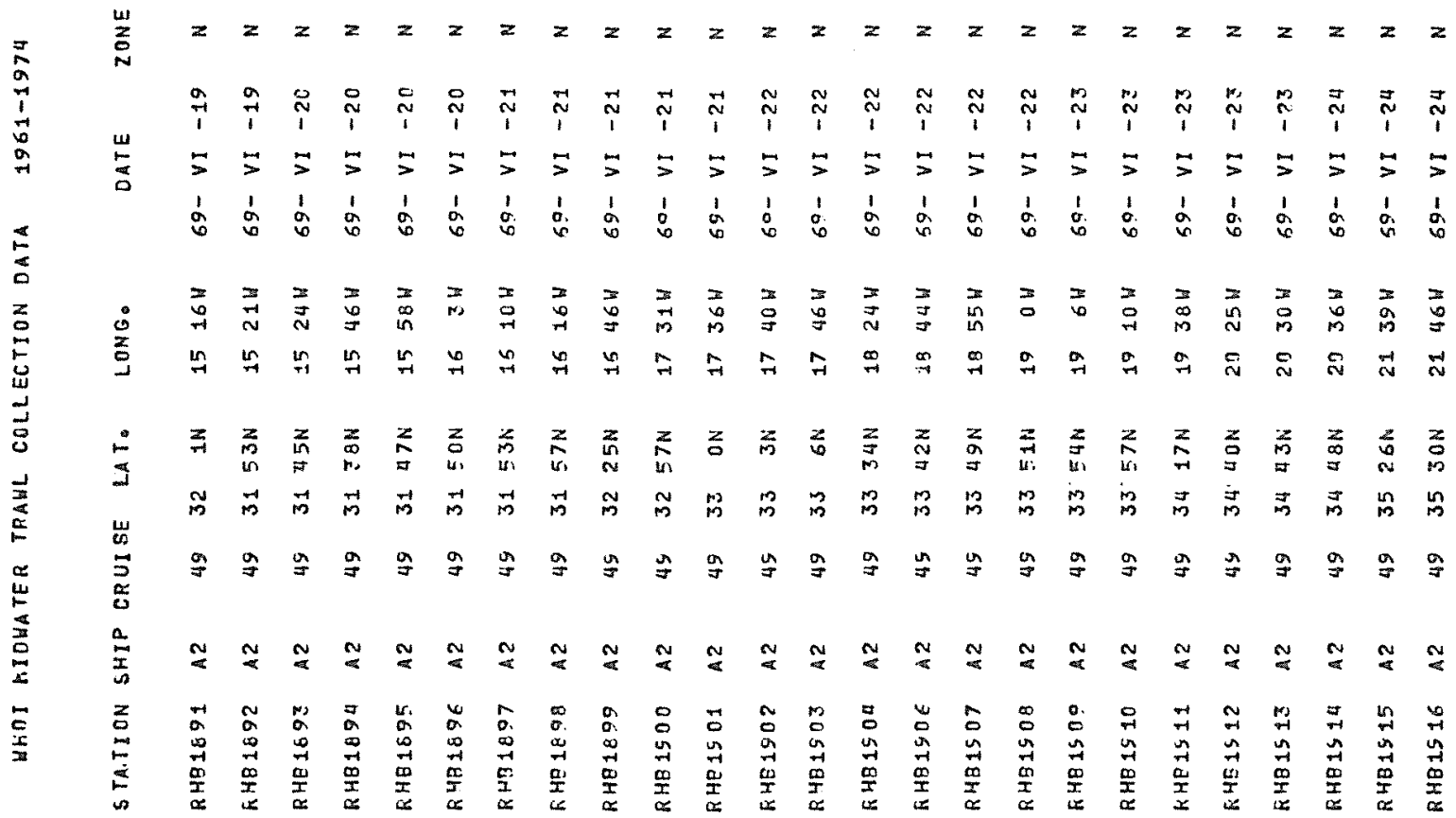


2
$\vdots$
0
0

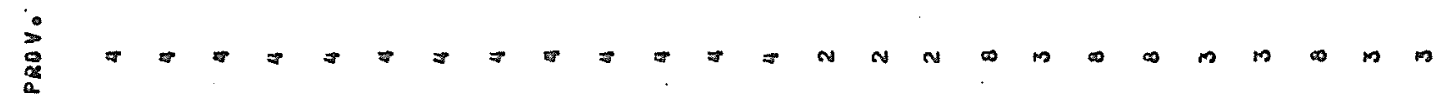

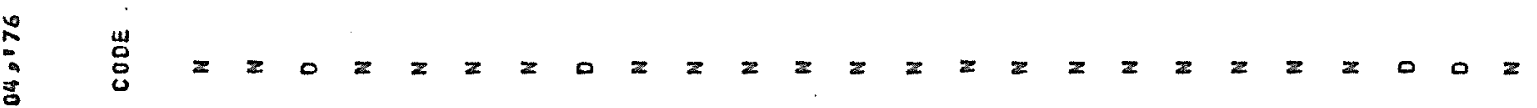

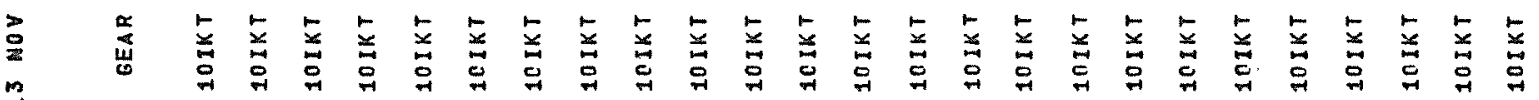

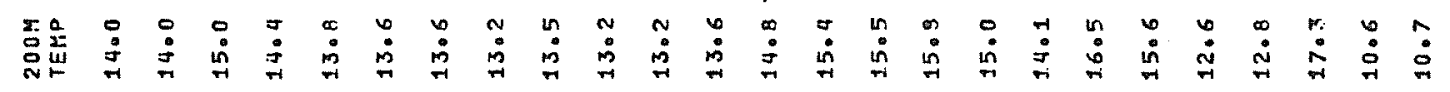

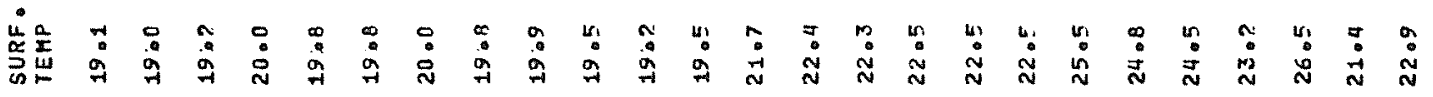

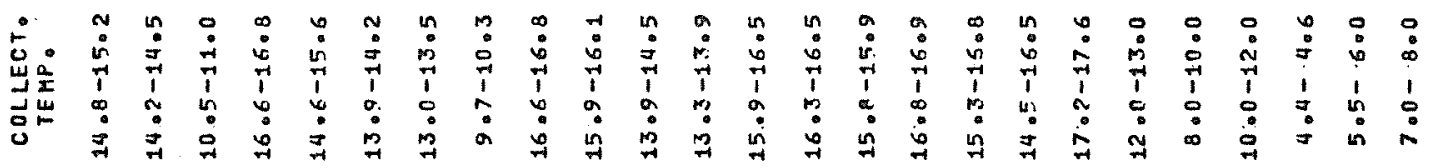

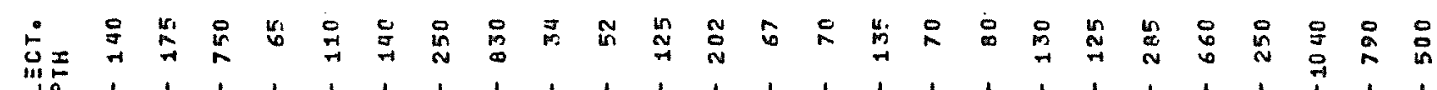

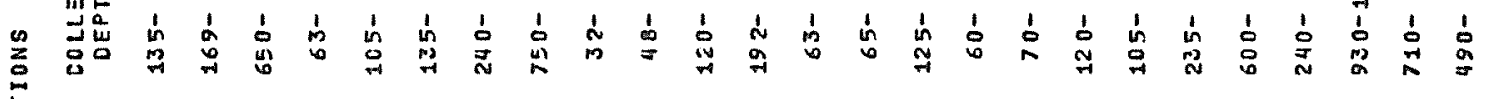
is

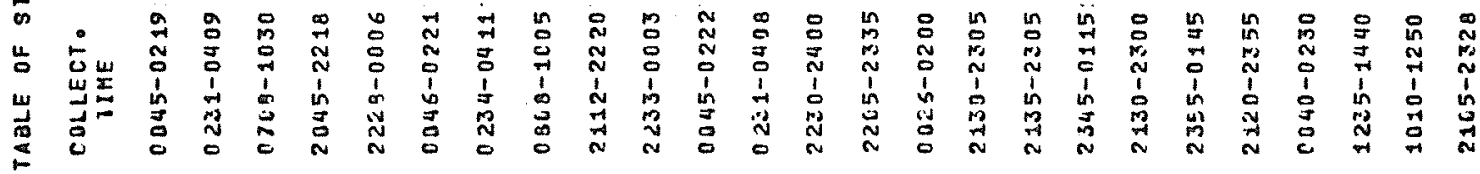

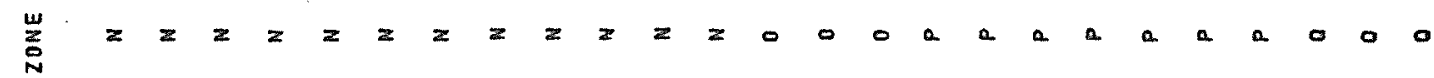

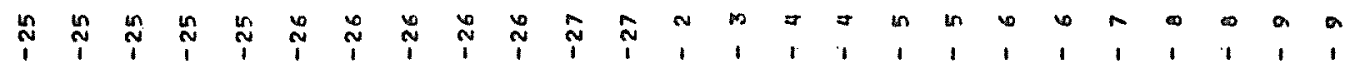

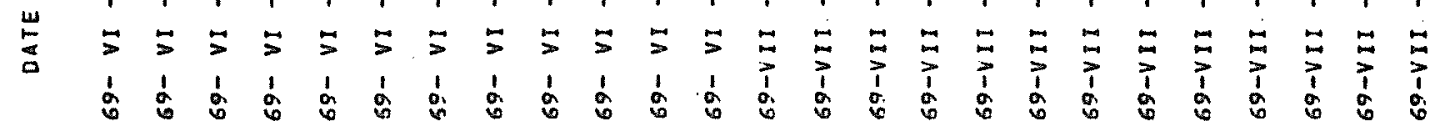

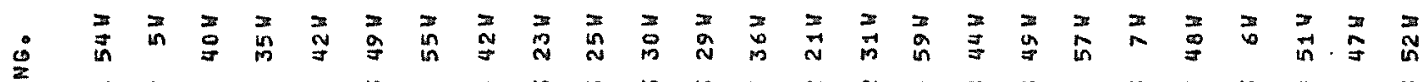

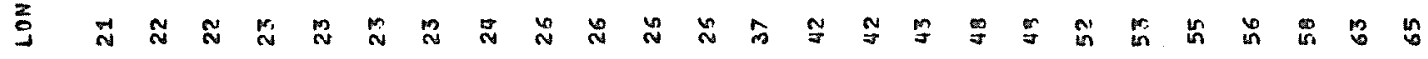

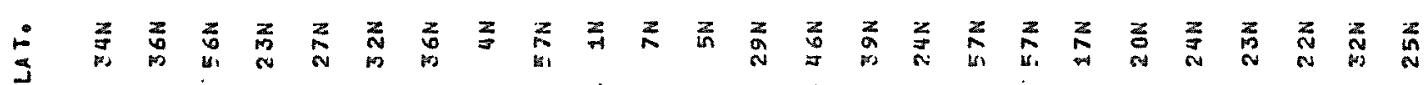

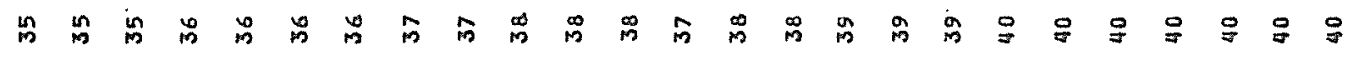

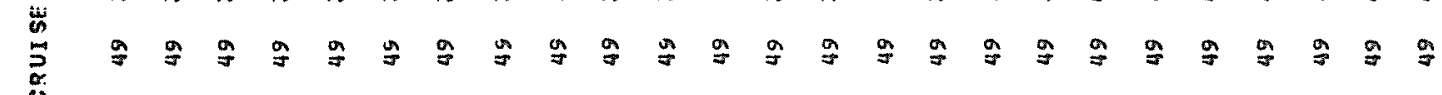

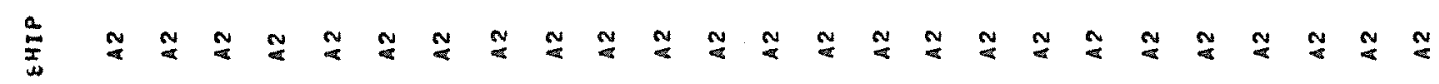

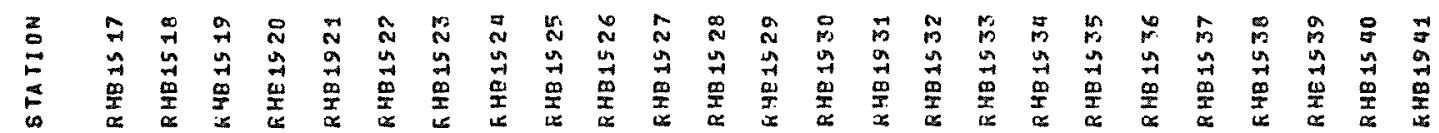


우

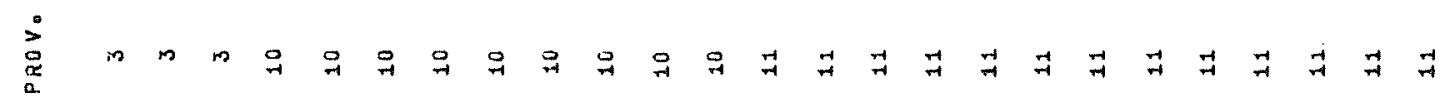

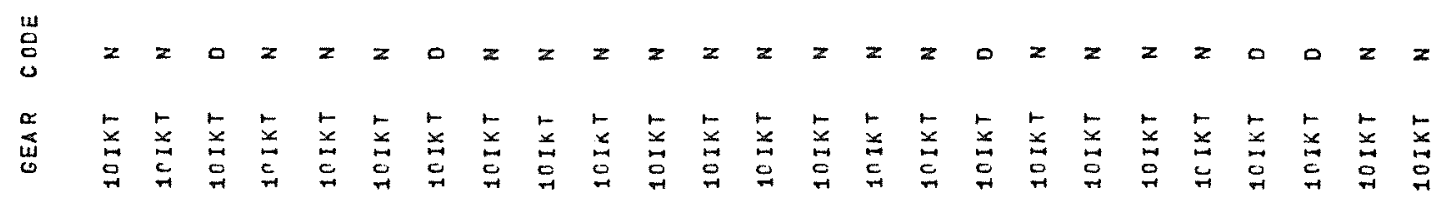

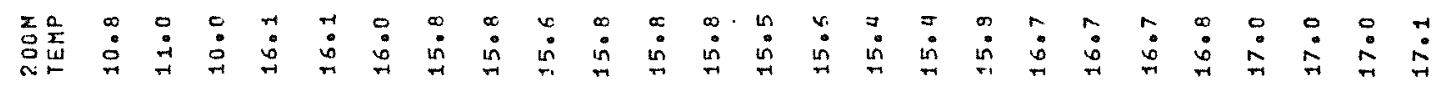

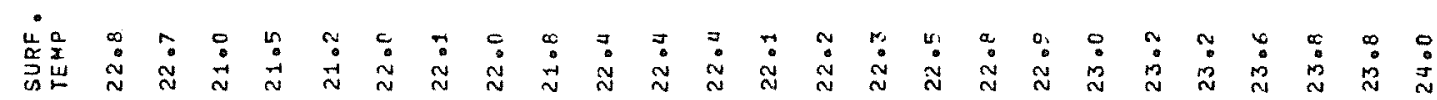

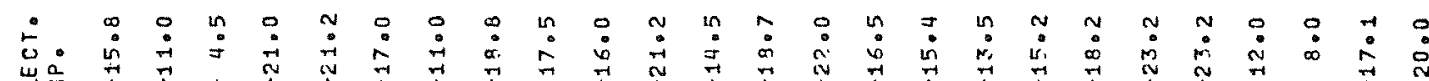

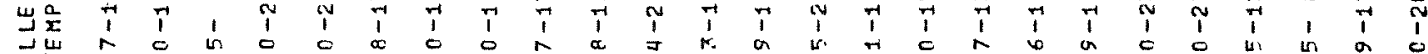

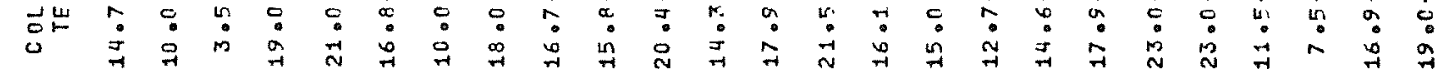

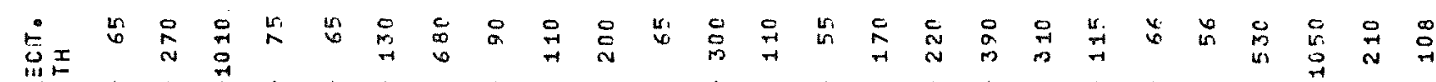

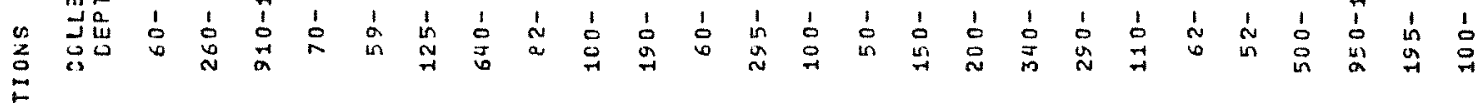

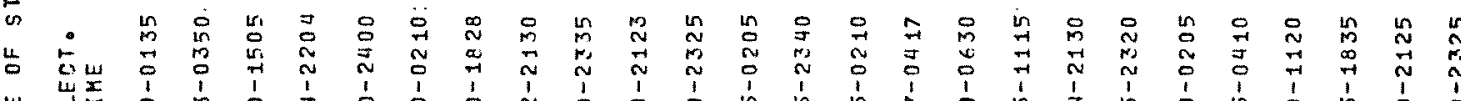

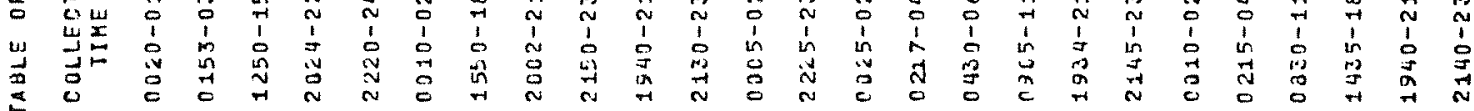

岩 000 N

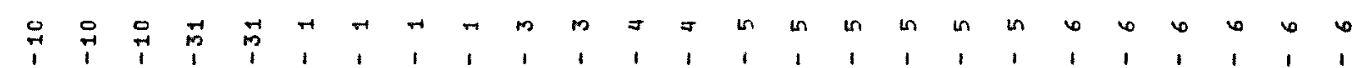

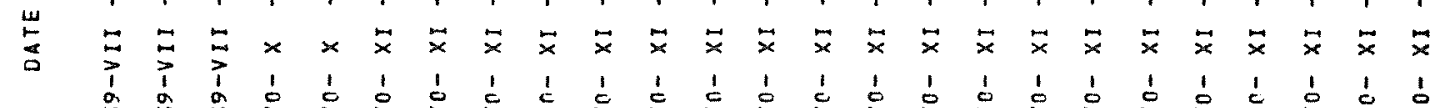

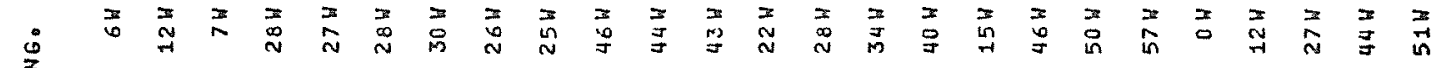

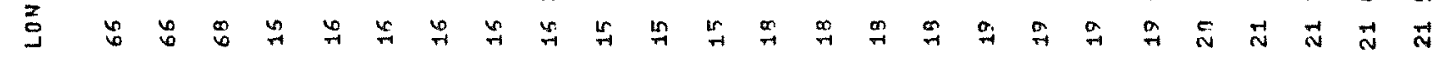

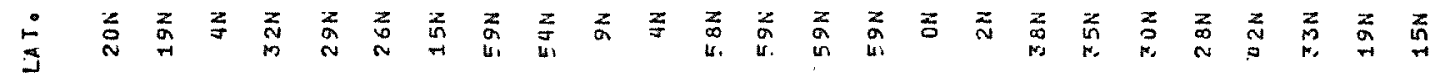

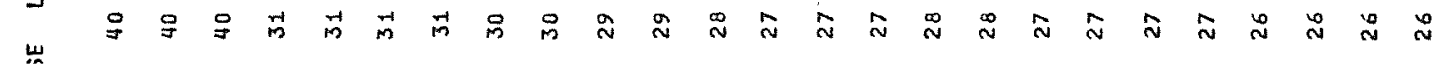

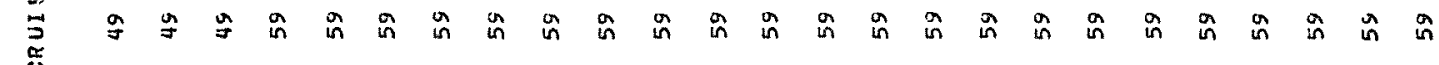

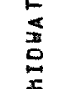

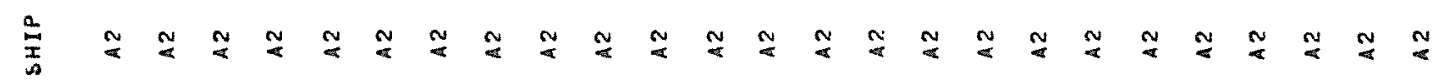

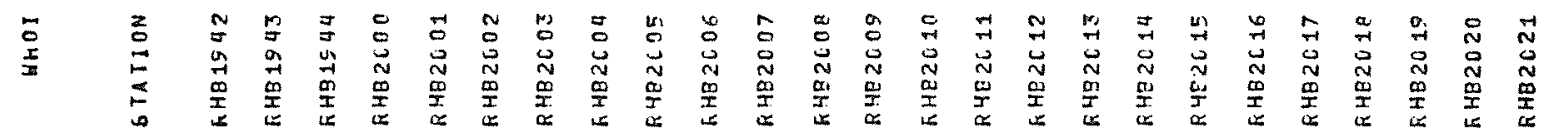


ה

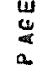

齐

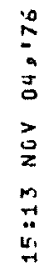

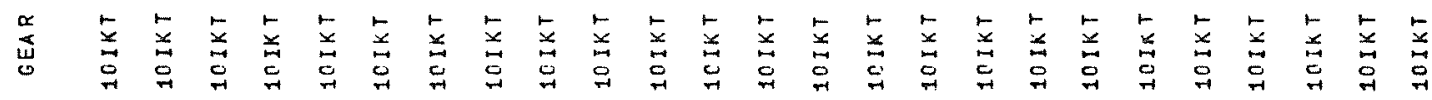

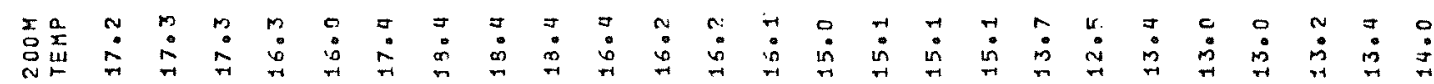

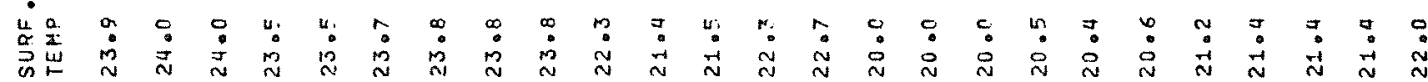

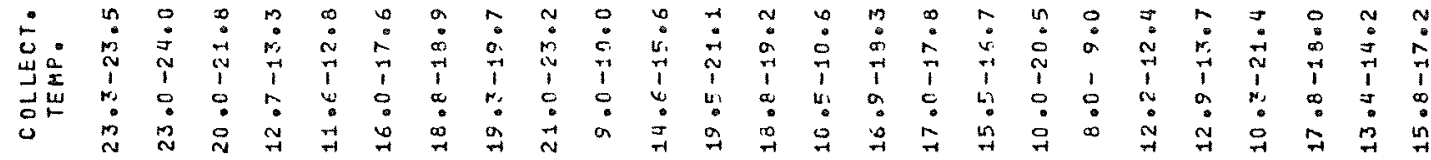

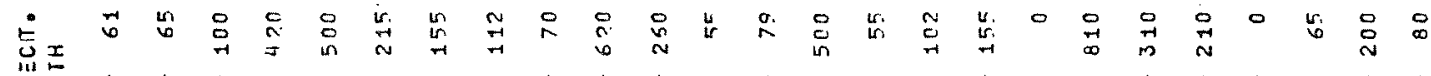

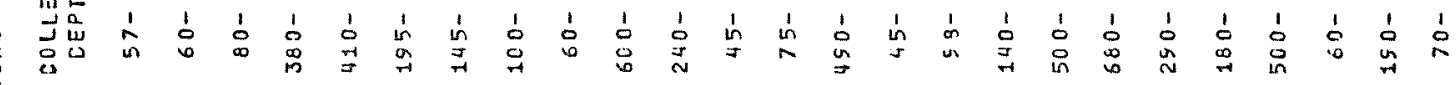
$\underset{5}{5}$

u.

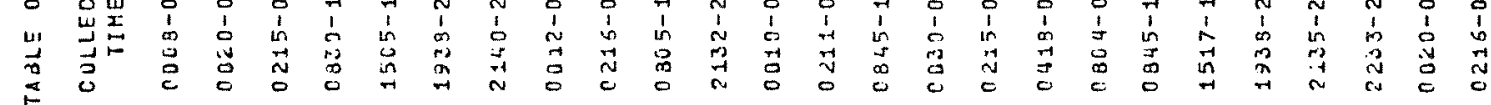

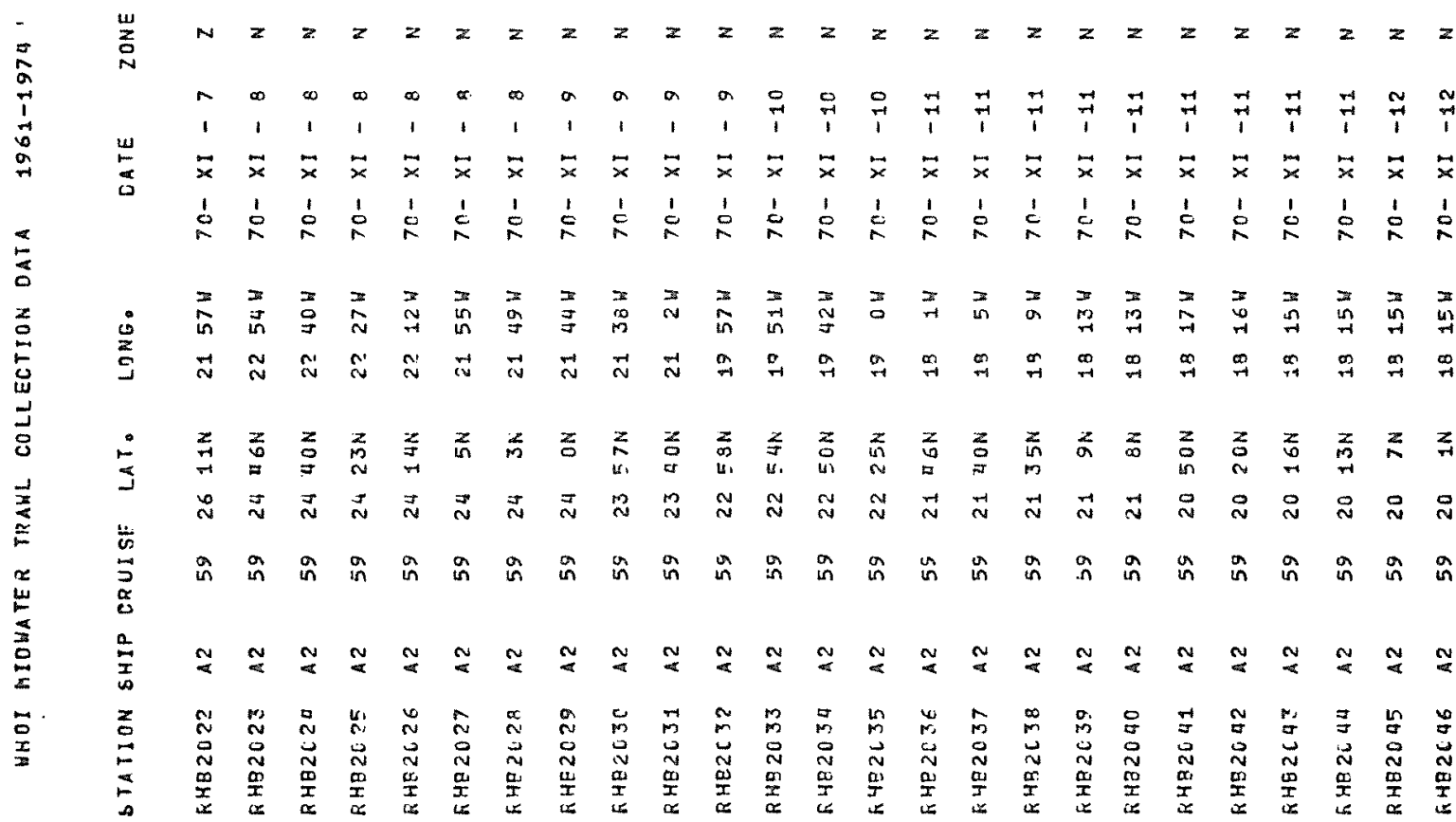


N
$u$
0
0
0
$\vdots$
$\vdots$
$\vdots$
$\vdots$
$\vdots$
$z$
$z$
$\vdots$
$:$

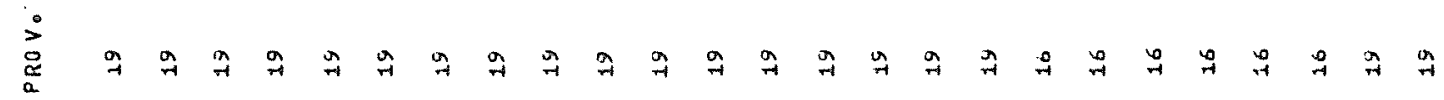

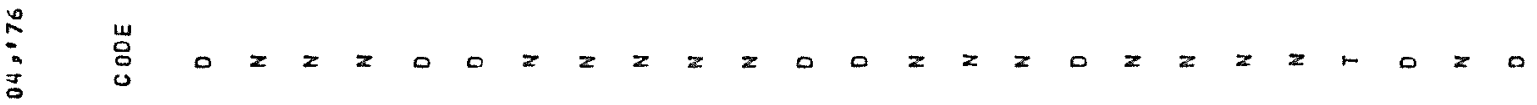

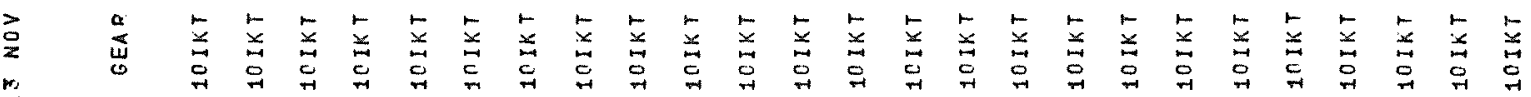

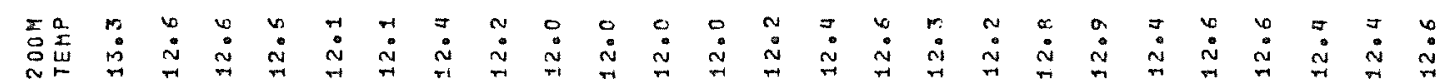

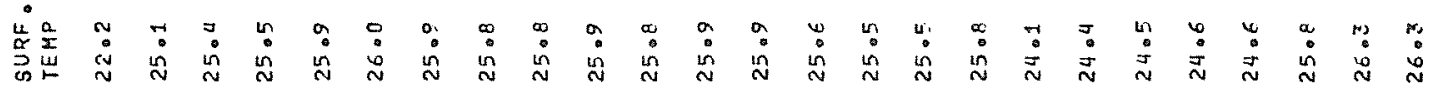

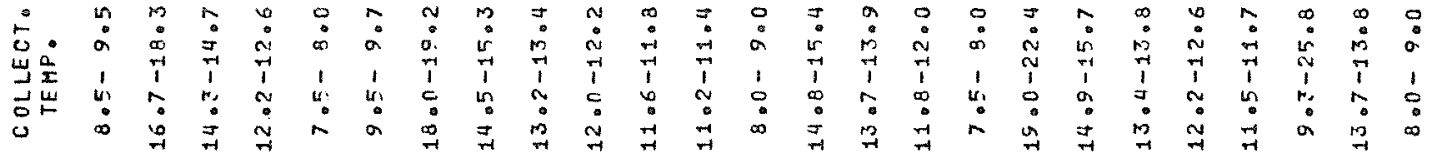

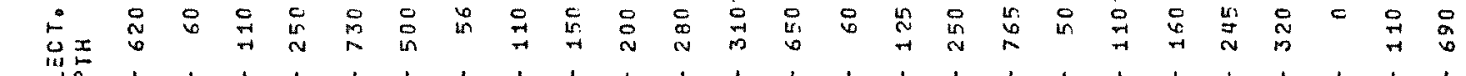

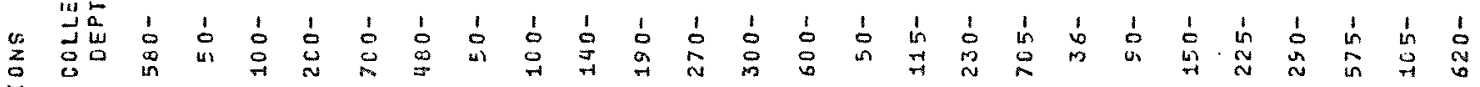
点

L

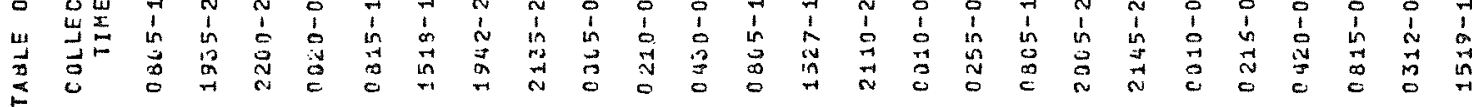

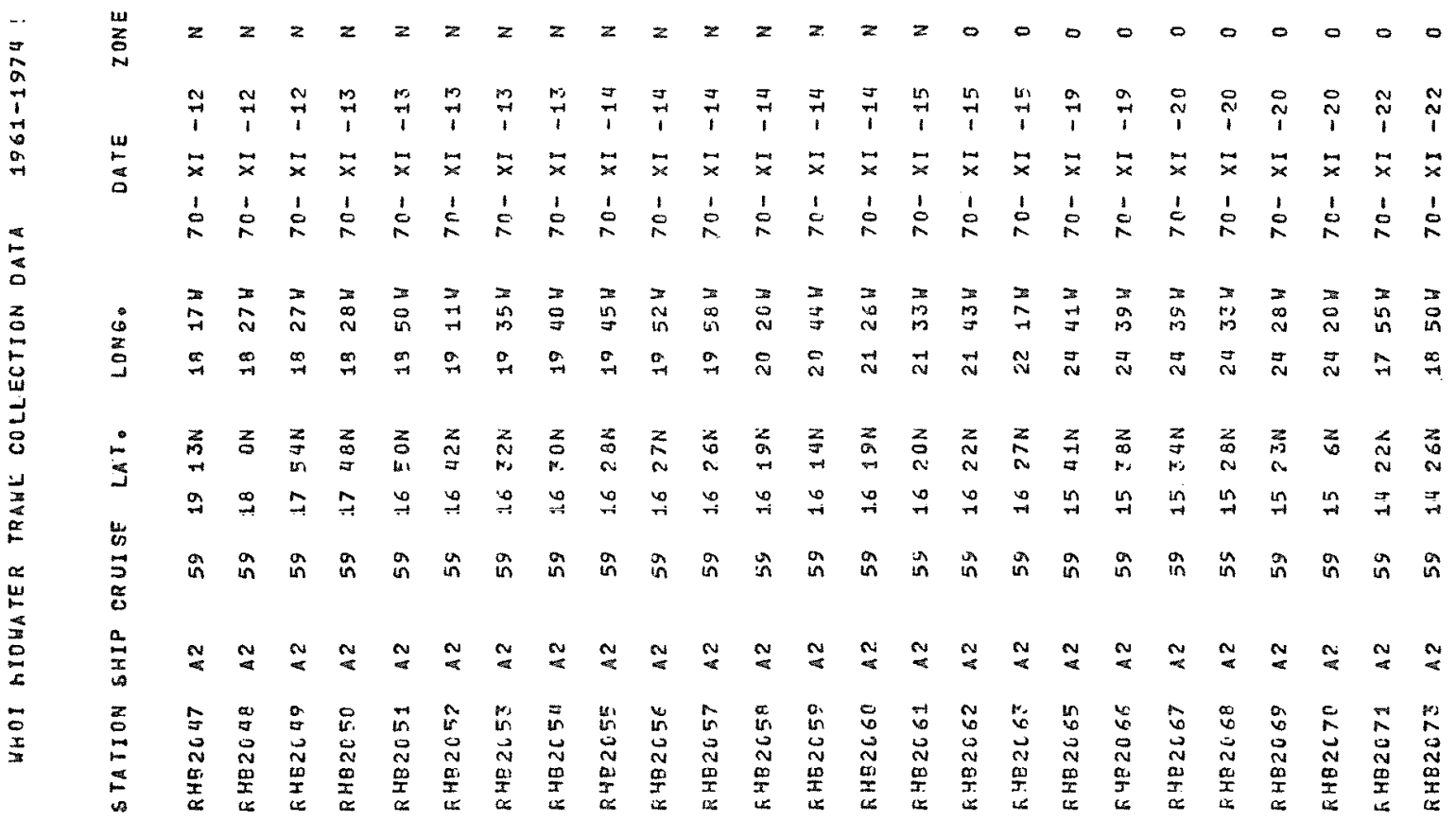




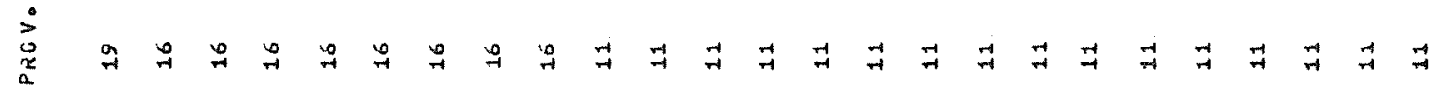
†

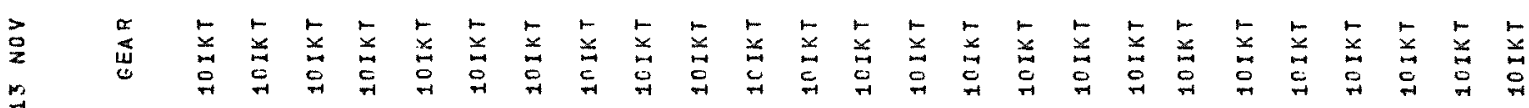

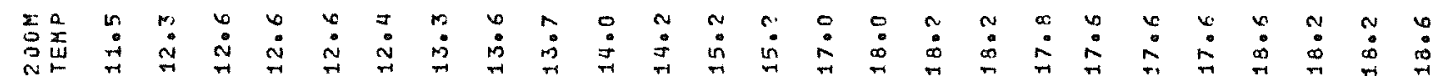

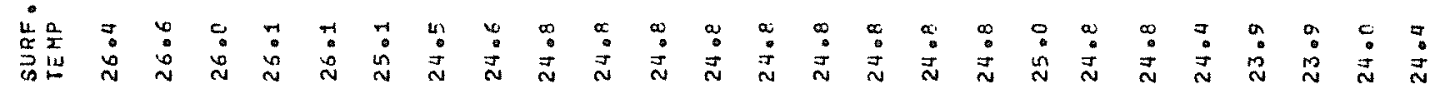

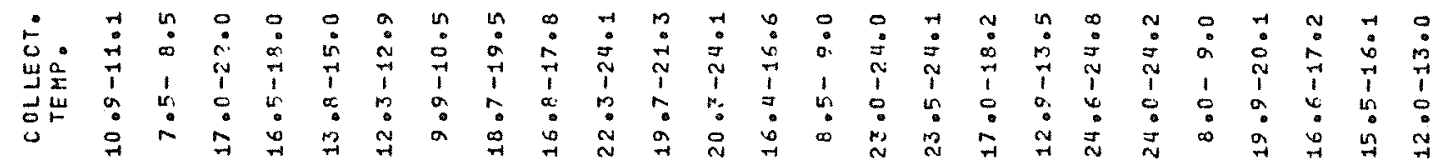
总玨只 只

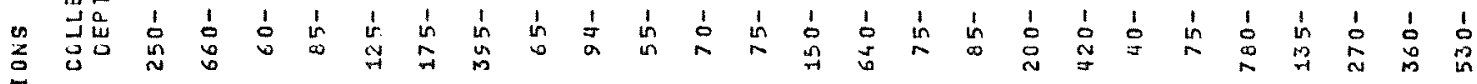
$\underset{⿱ 乛}{*}$

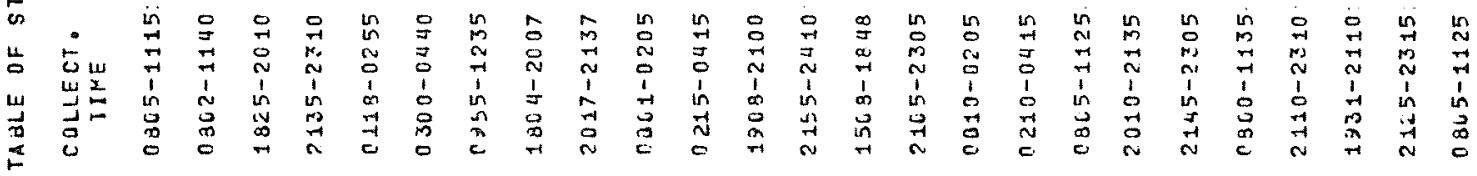

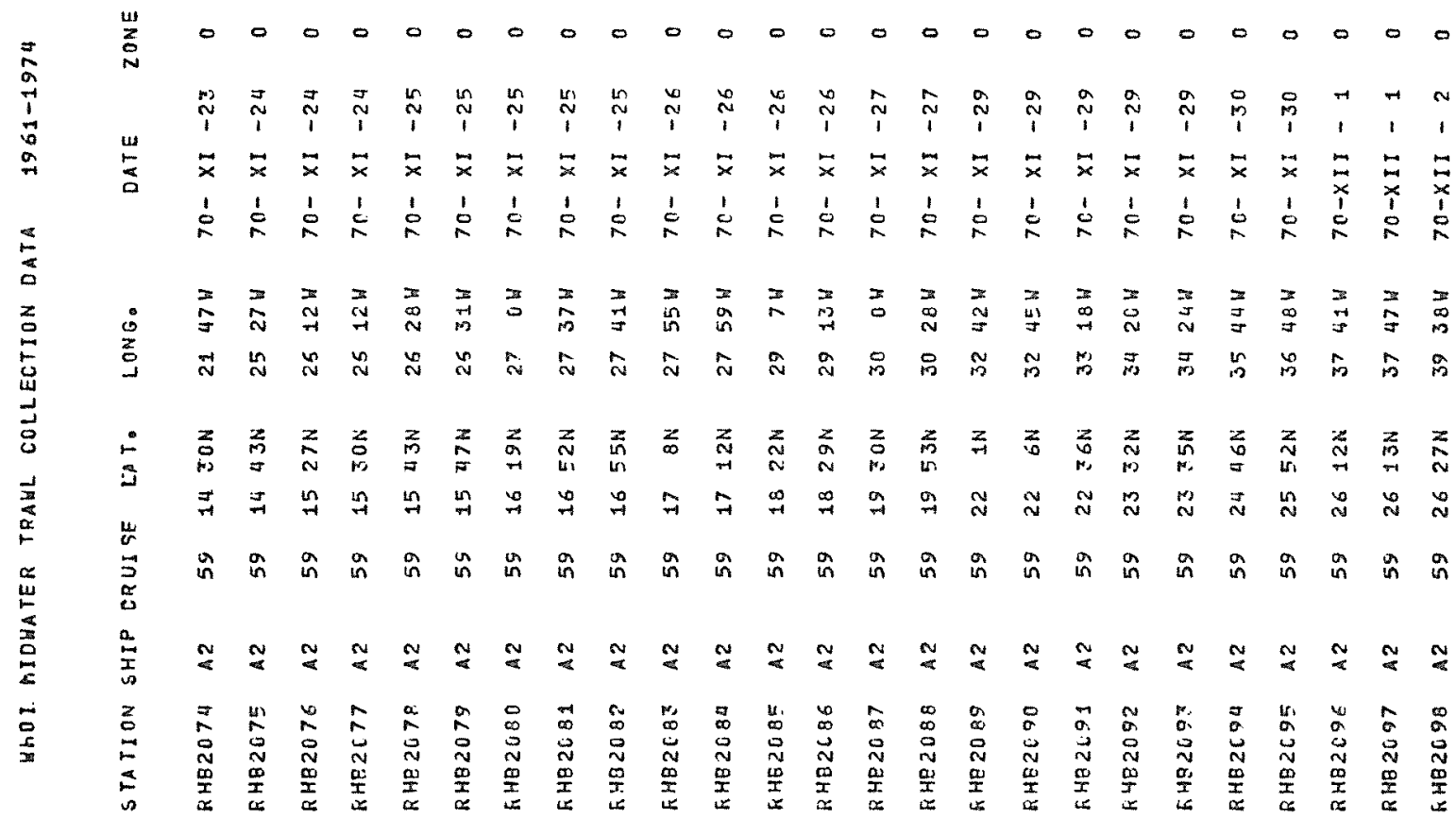




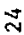

㖁

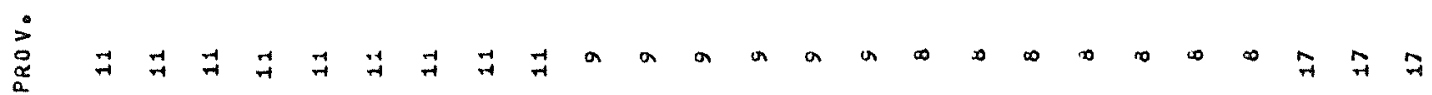

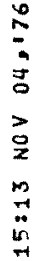

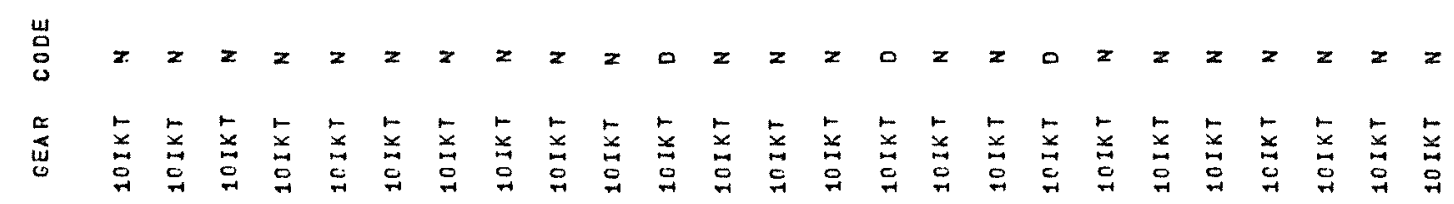

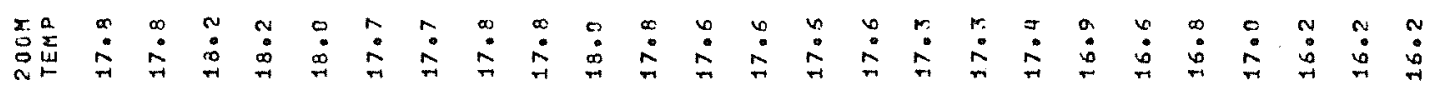

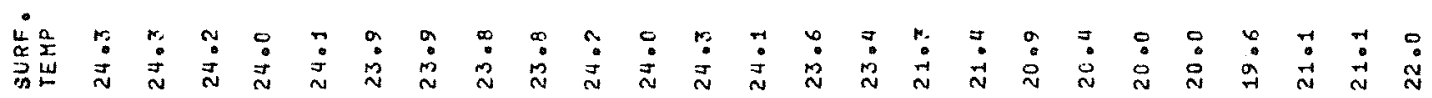

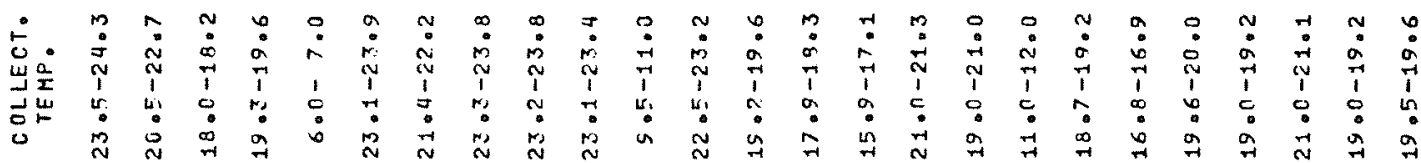

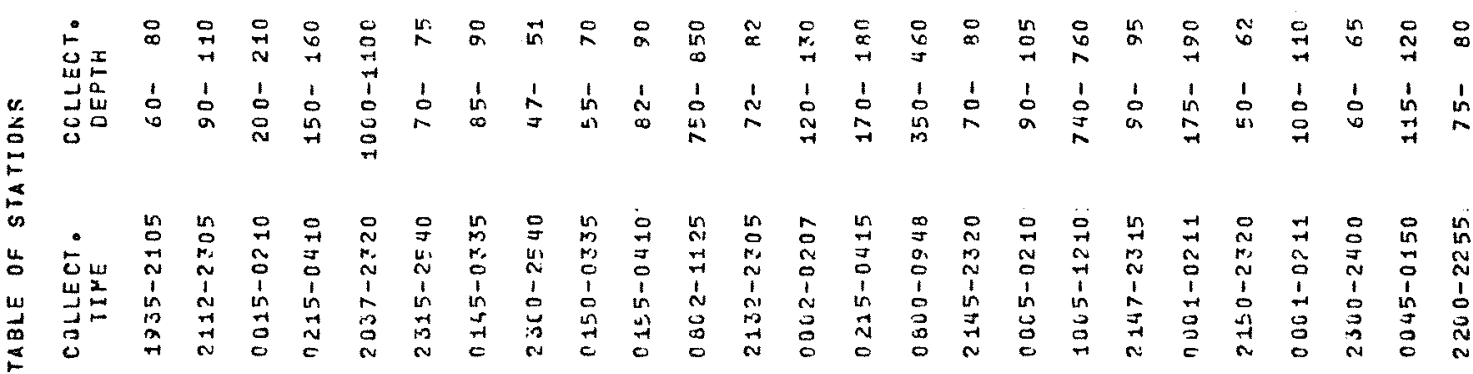

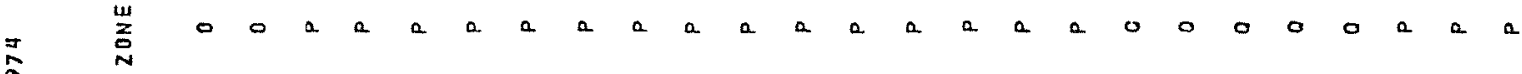

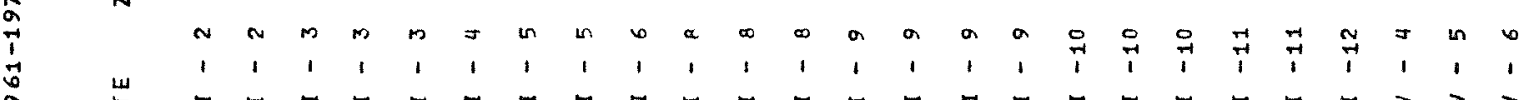

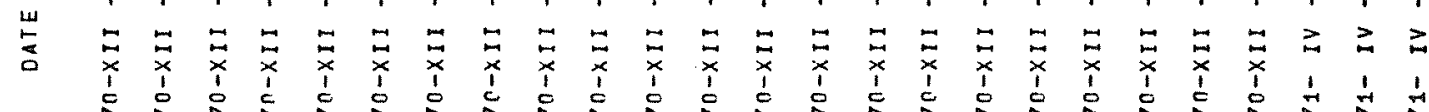

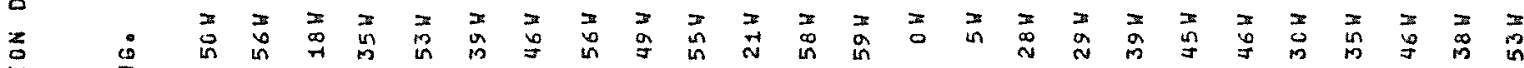

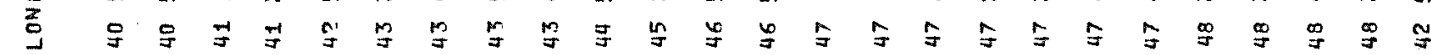

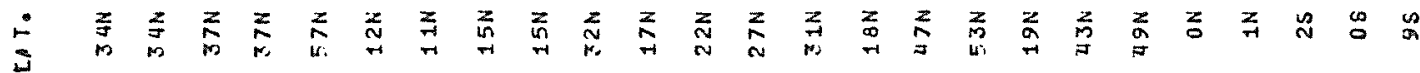

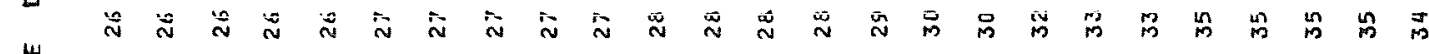

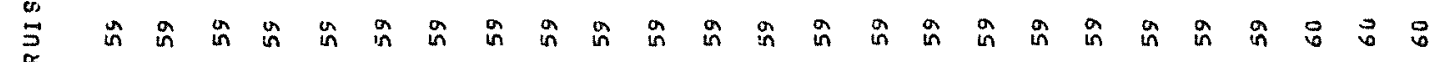

㽦

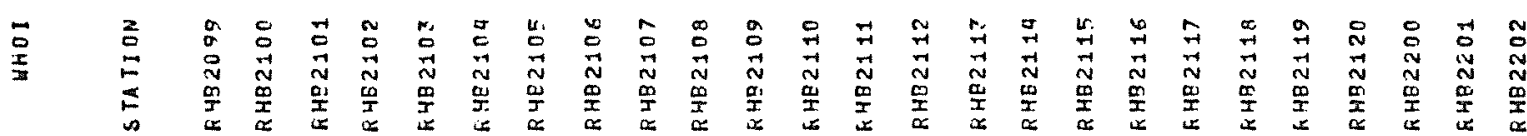




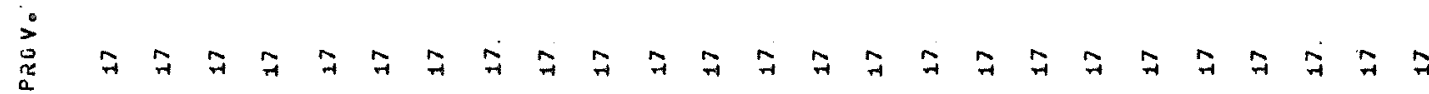
崖

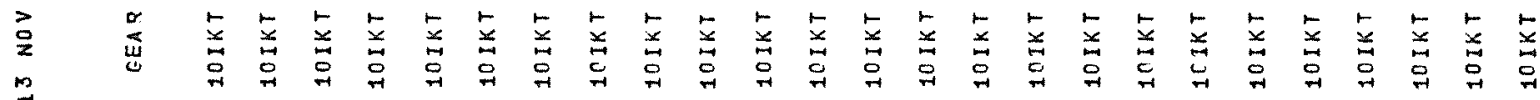
$\ddot{4}$

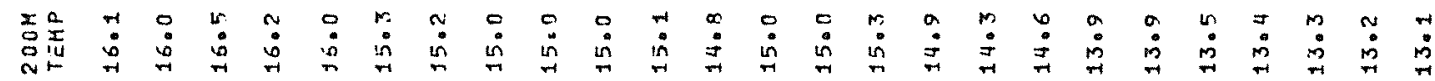

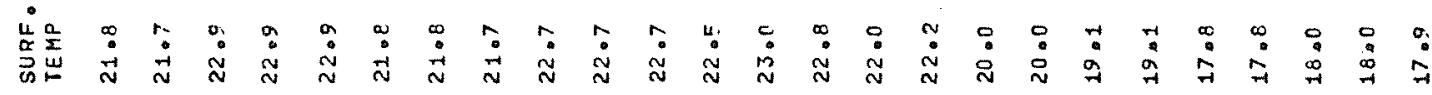

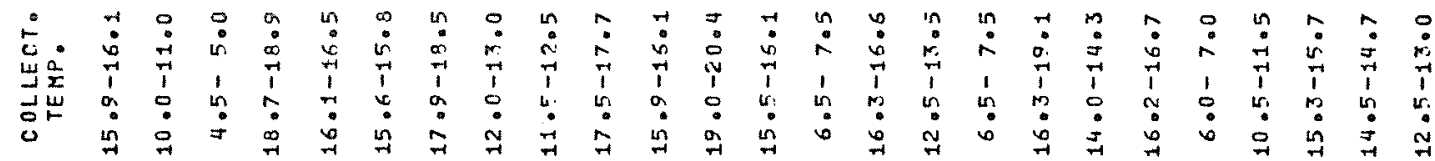

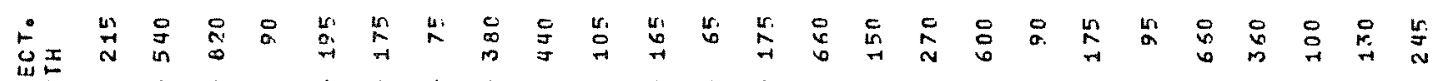

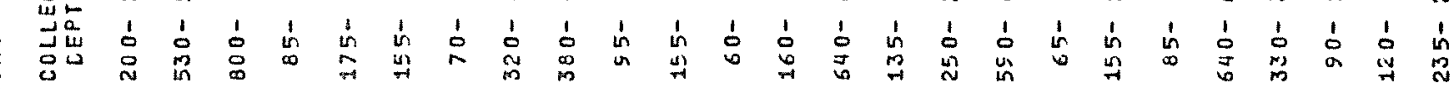
s

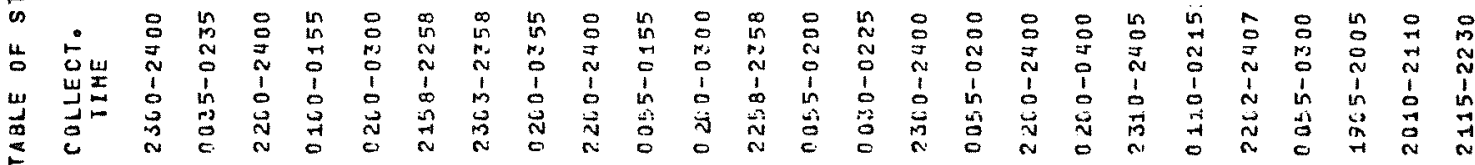
$=$

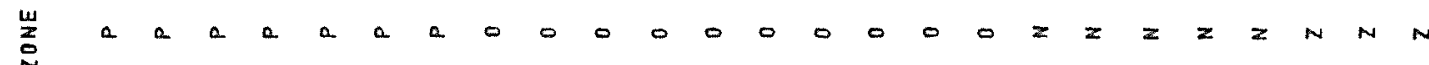

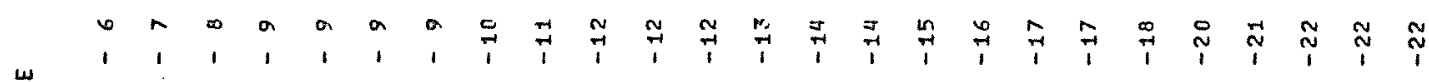

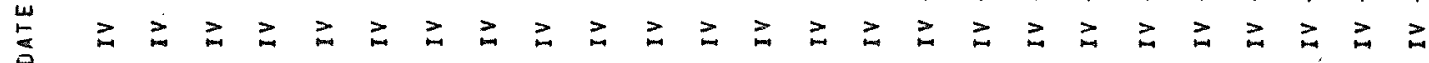

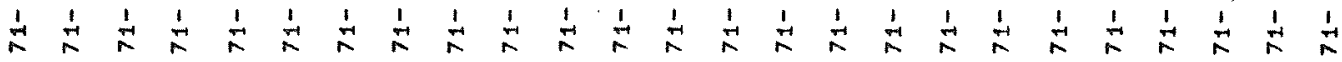




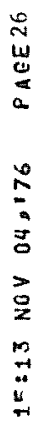

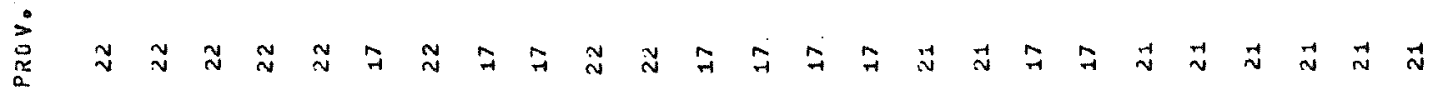

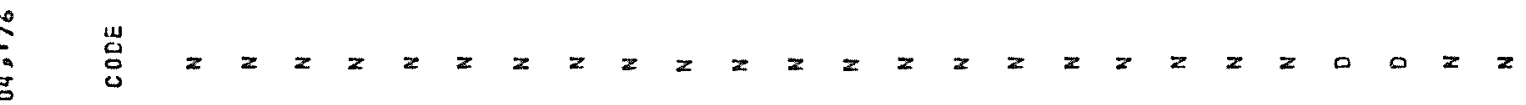
总 芯 点

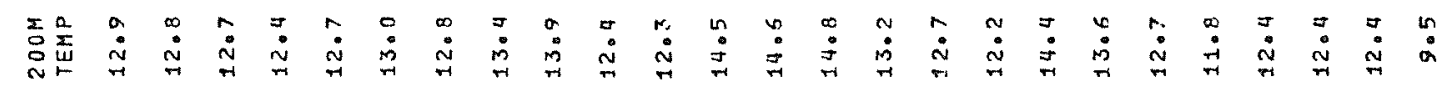

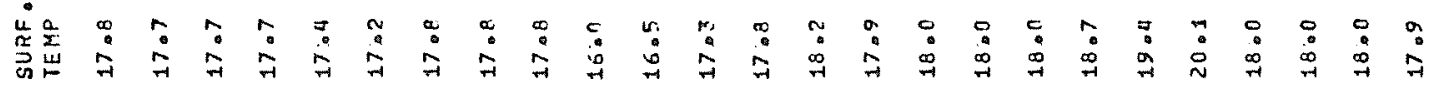

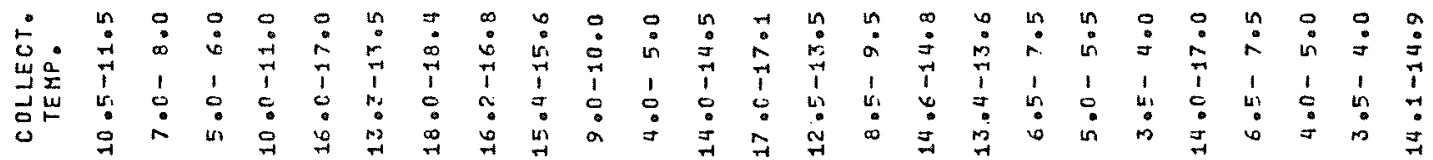

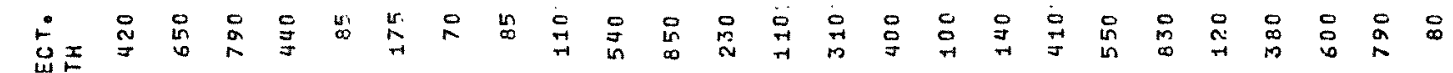

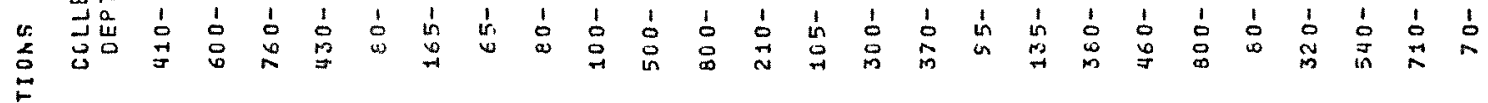
is

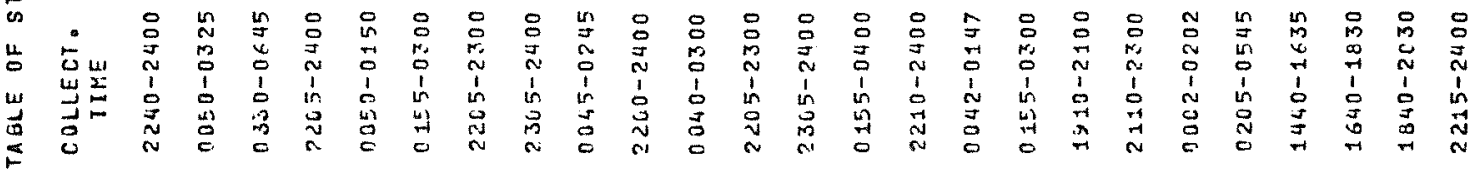

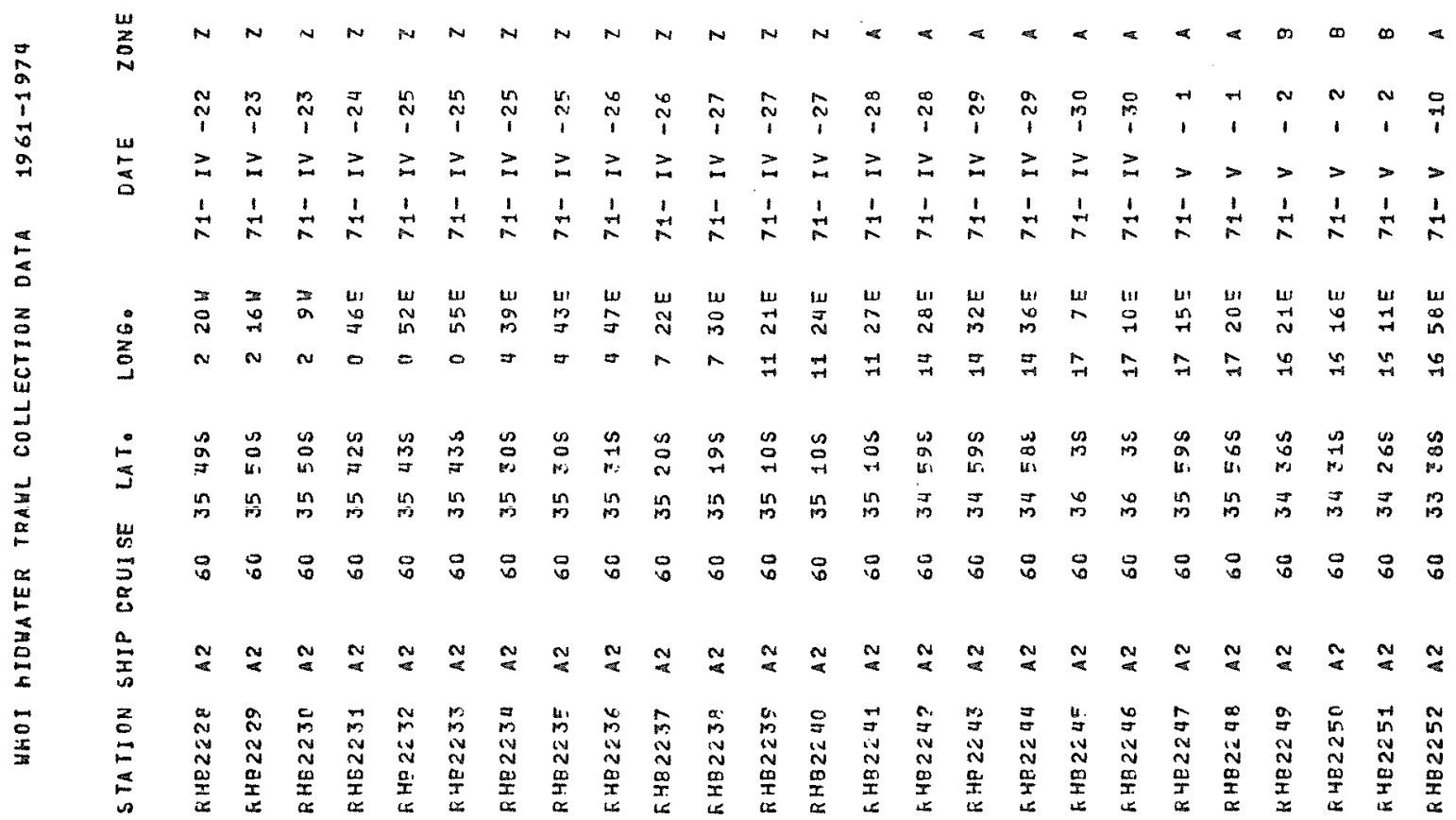




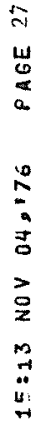

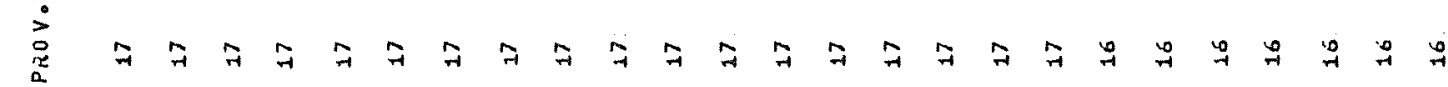

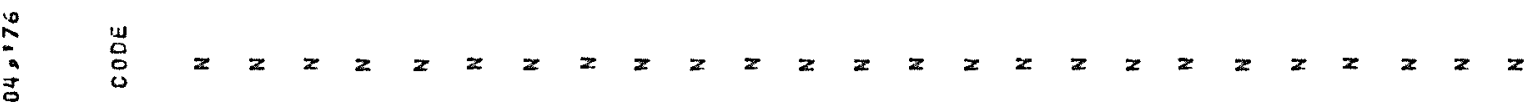

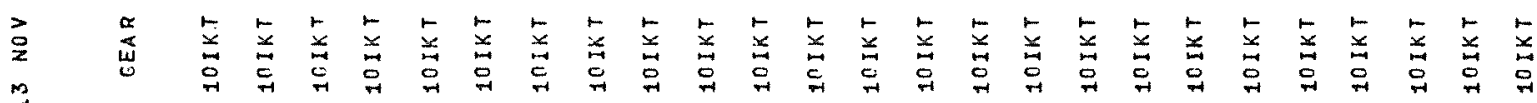

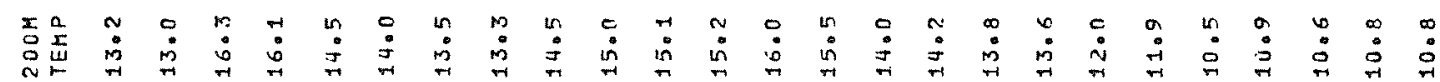

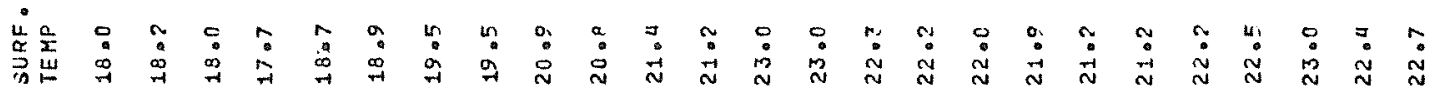

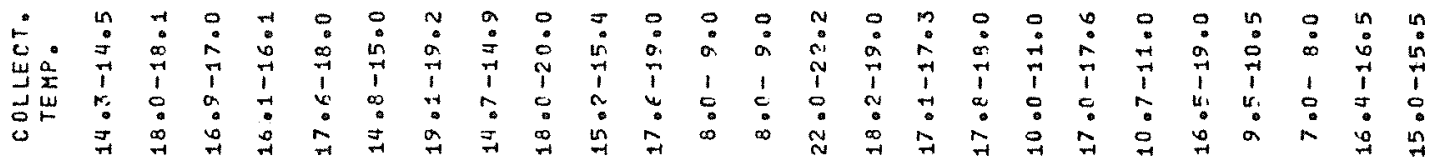

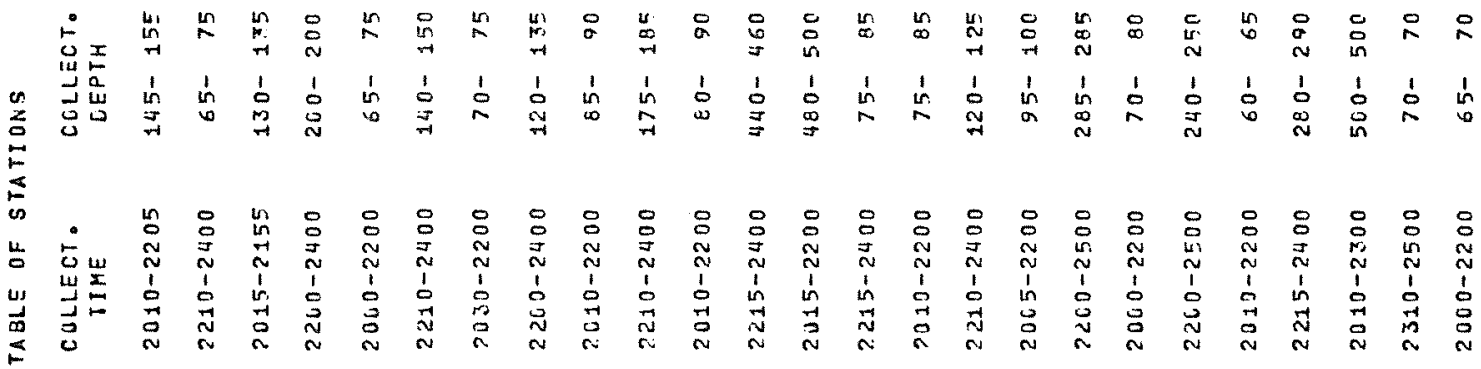

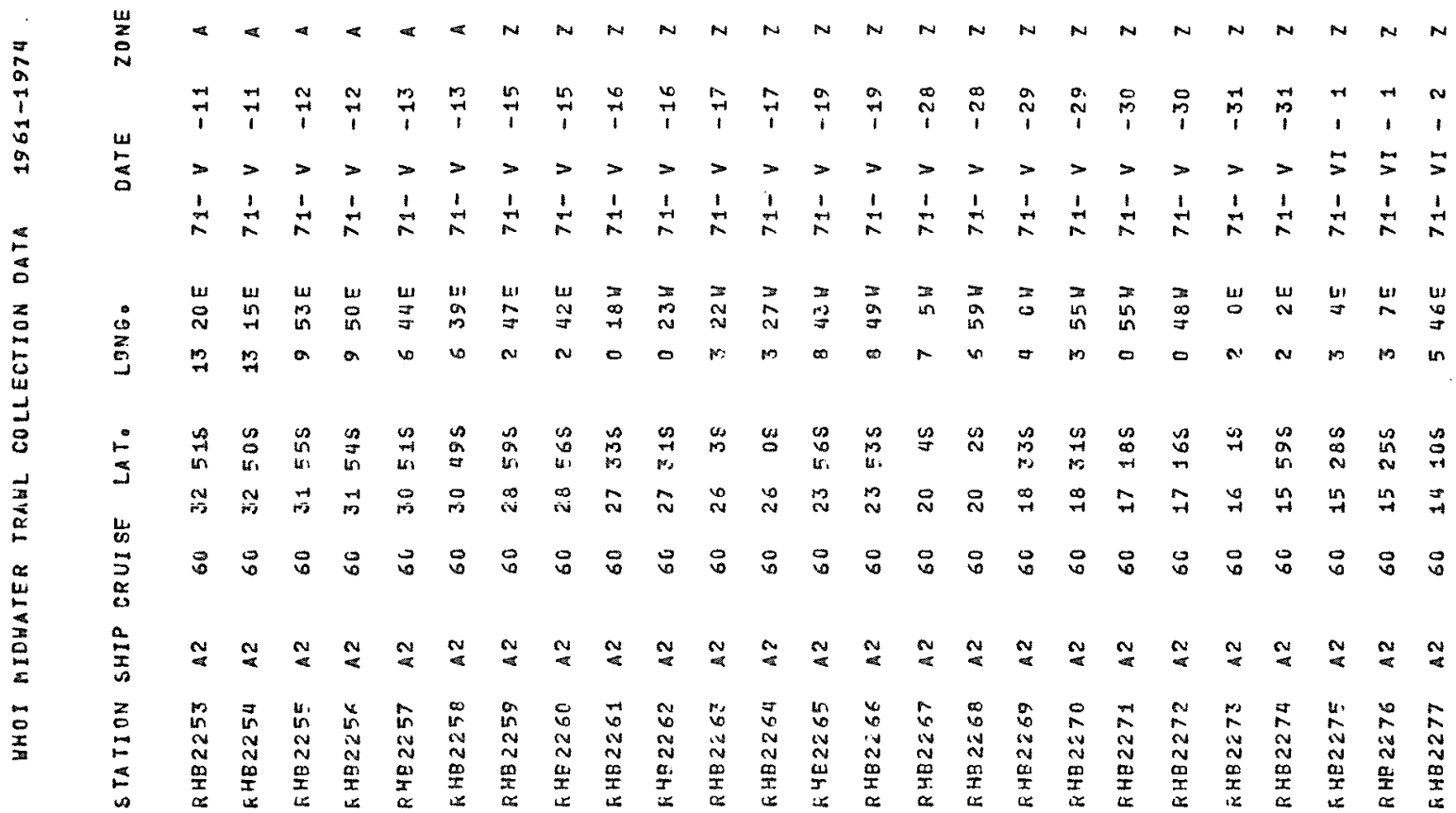


ก

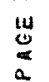

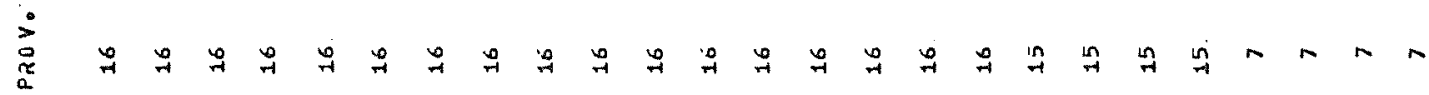

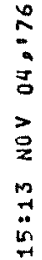

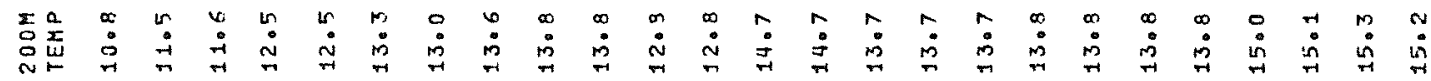

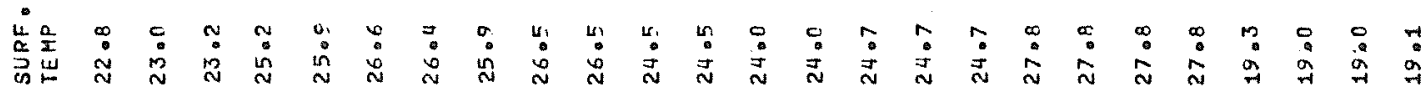

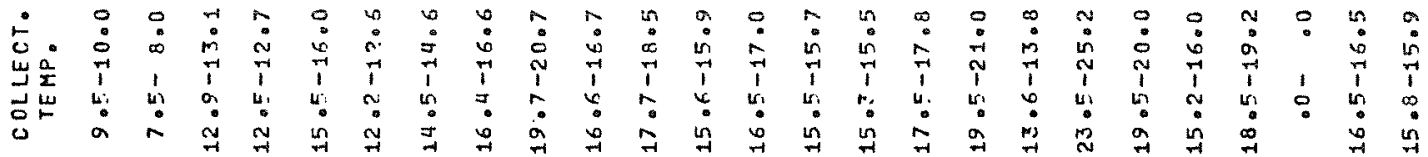

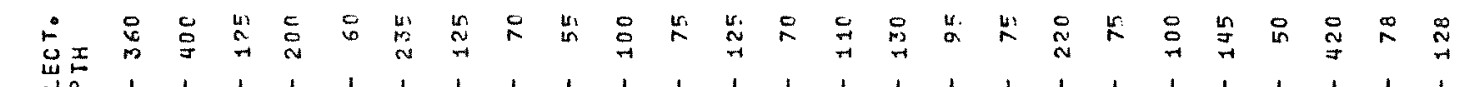

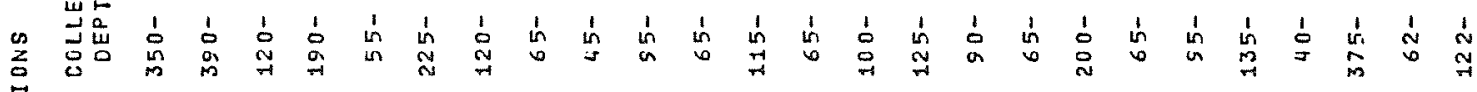
$\stackrel{5}{\infty}$

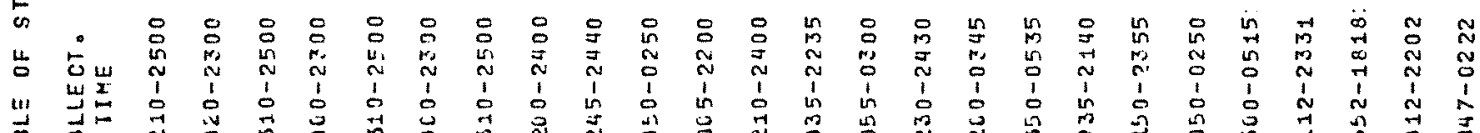

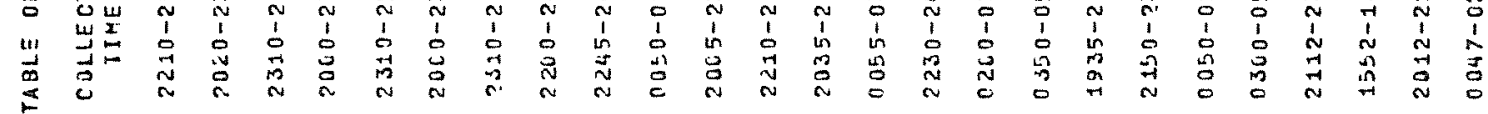

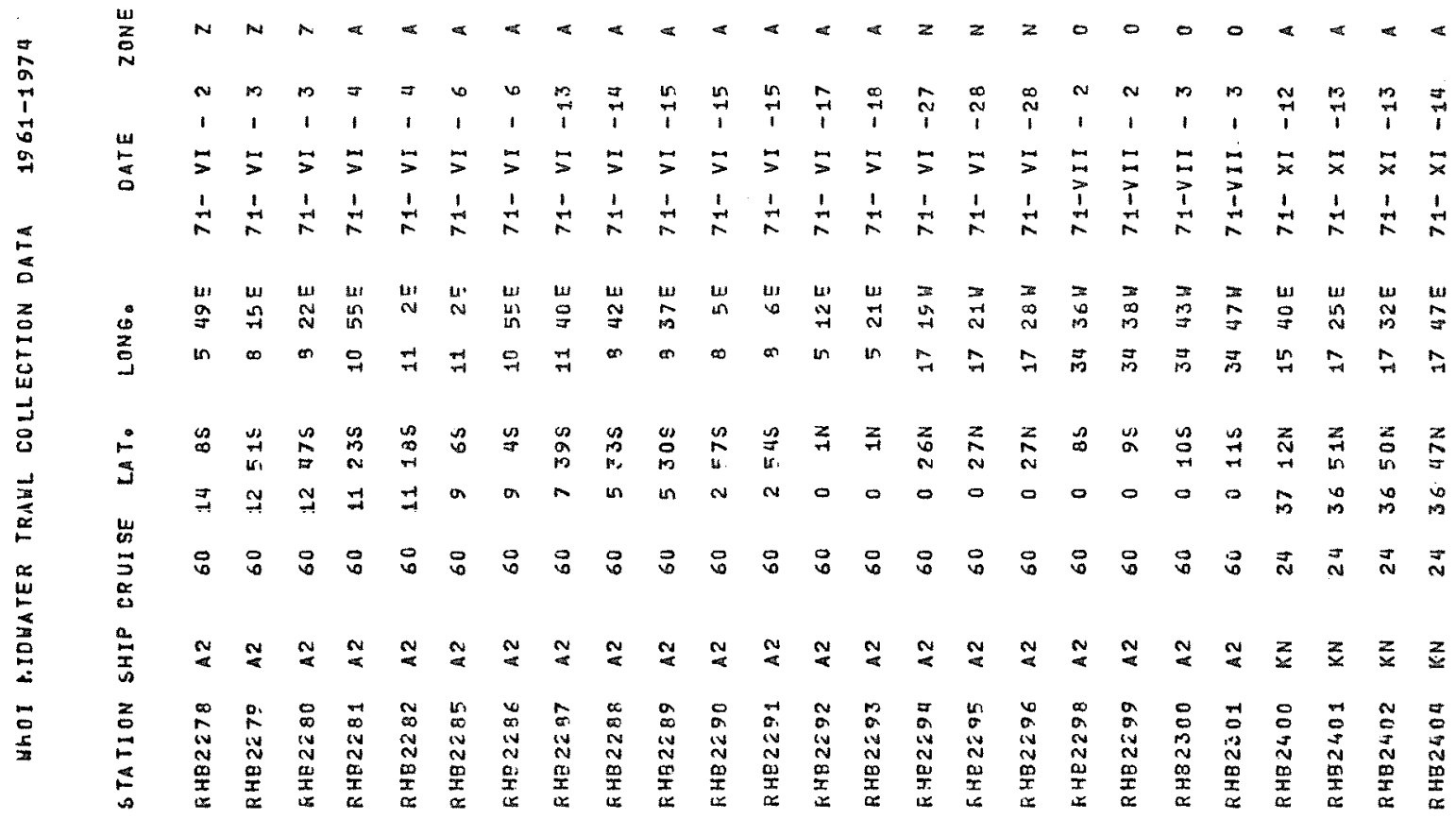




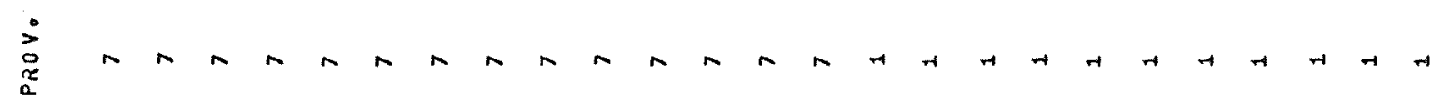

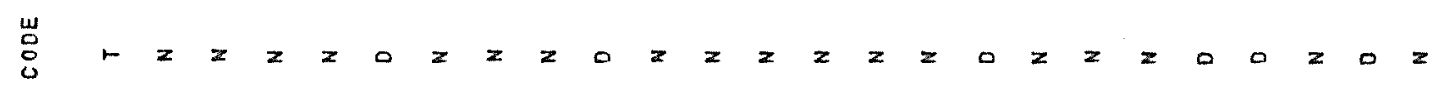

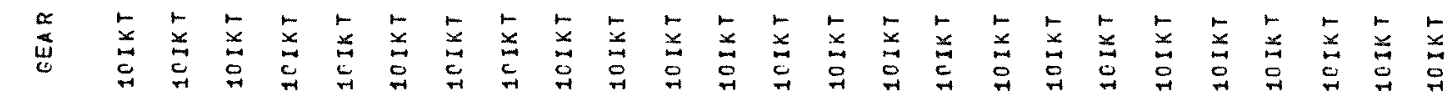

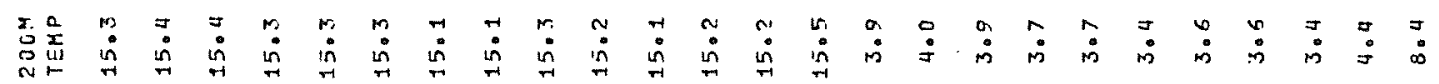

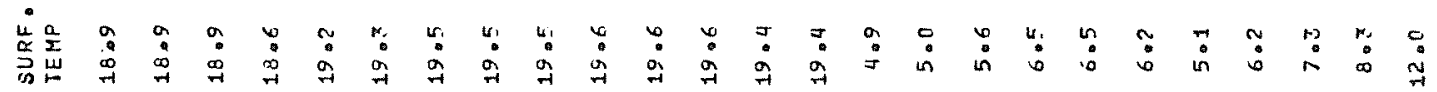

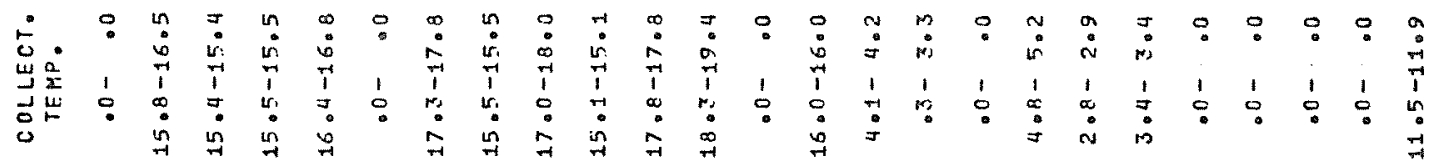

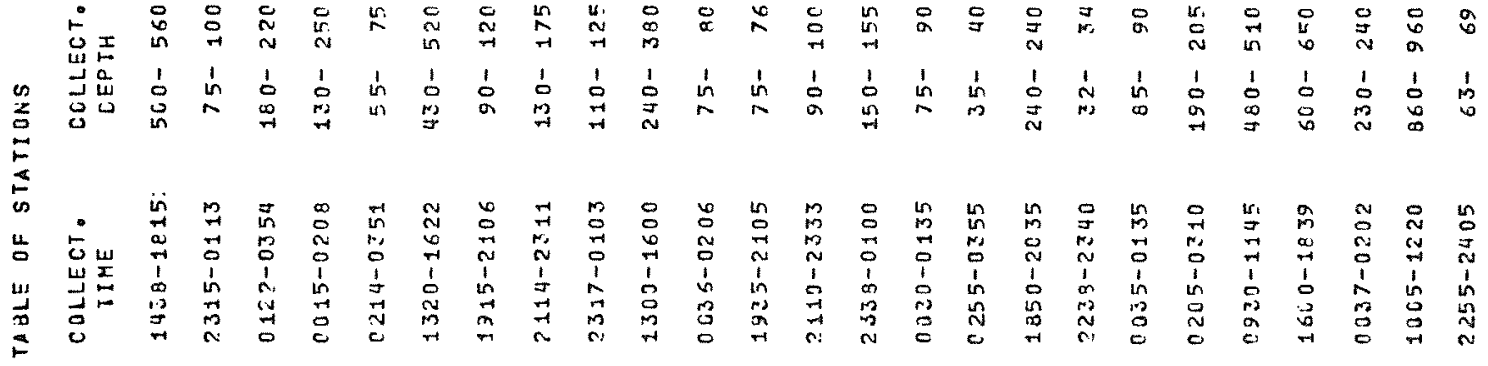

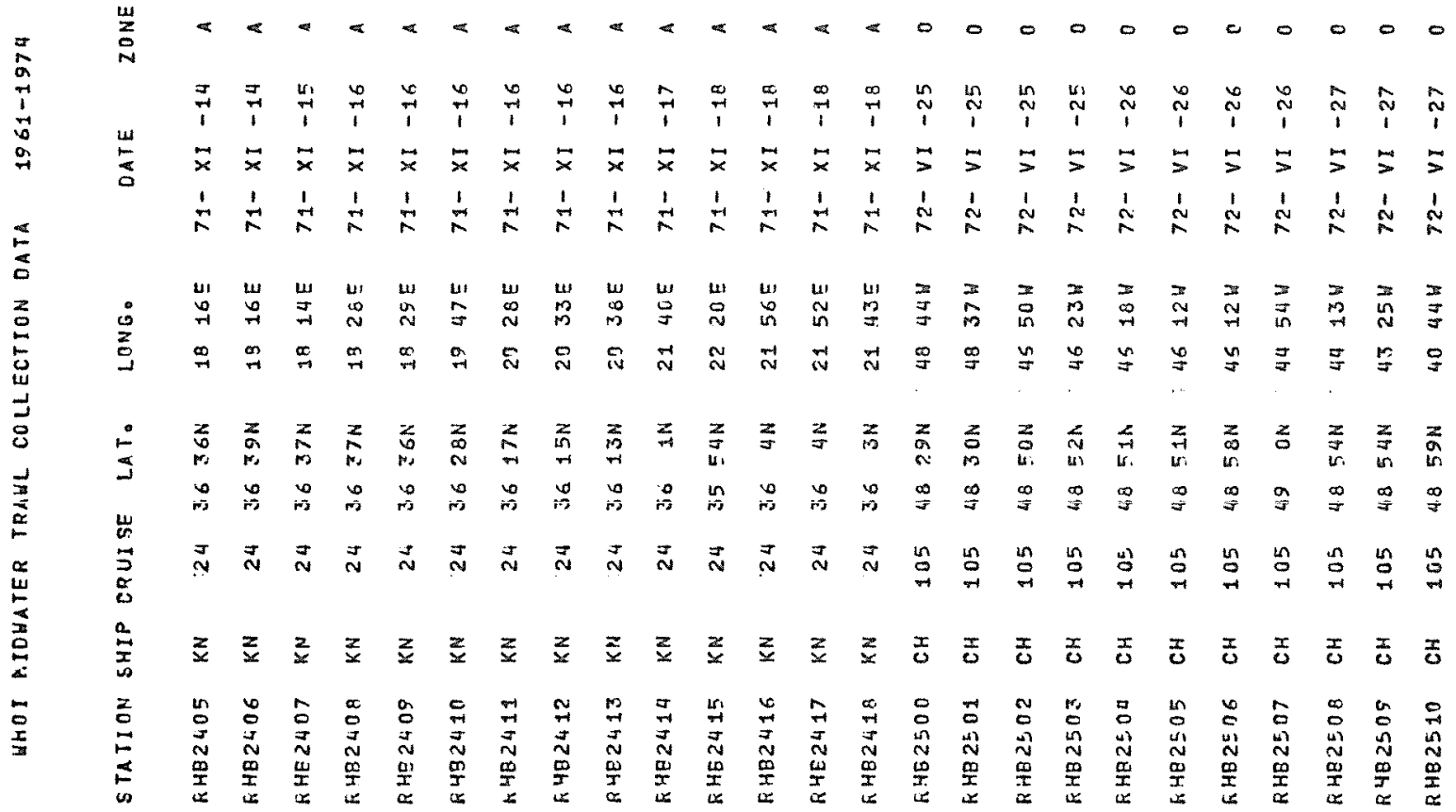


?

$\underset{\substack{w \\ \alpha}}{m}$

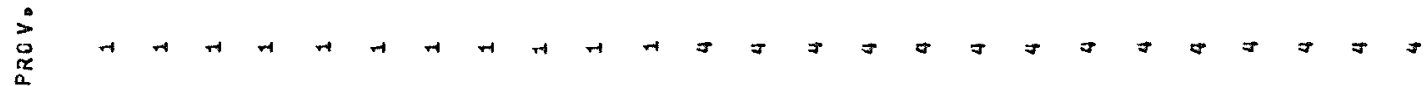
:

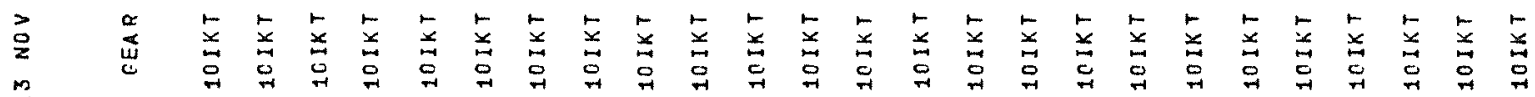

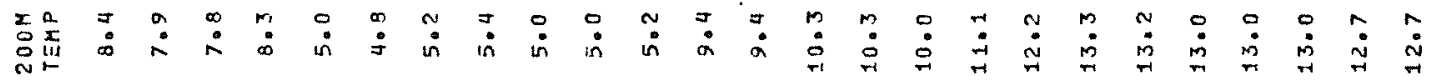

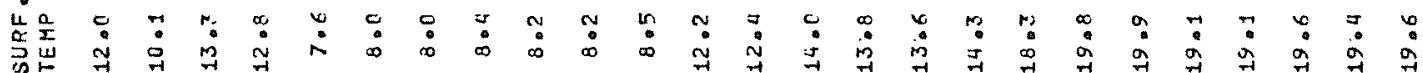

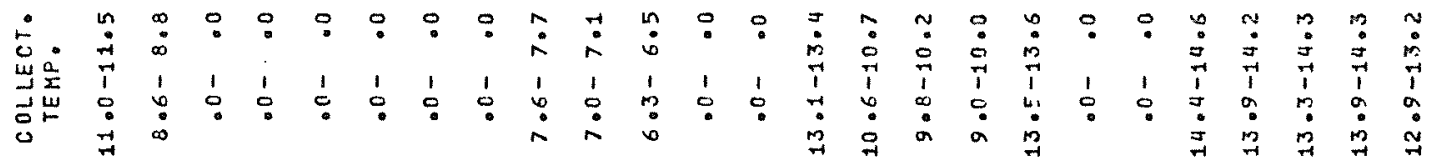

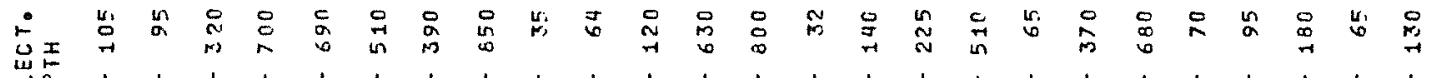

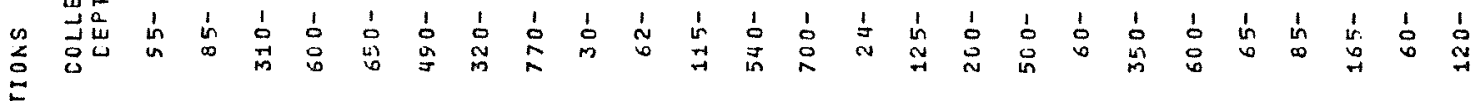
5

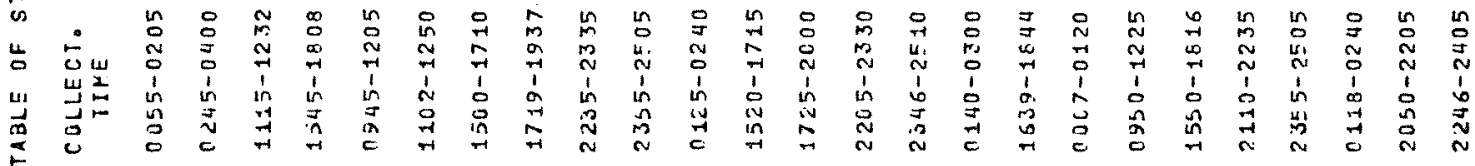

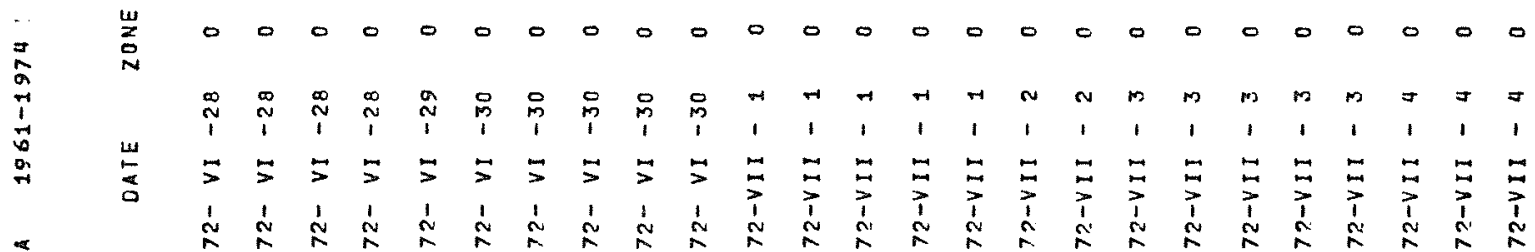

$\stackrel{5}{0}$ z

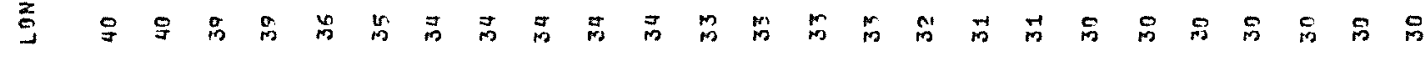

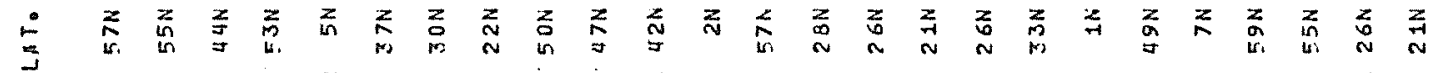

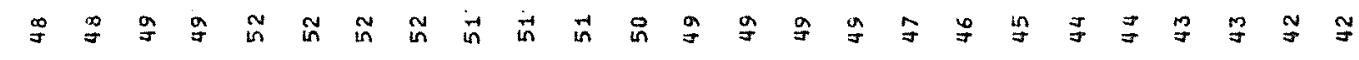
蓠

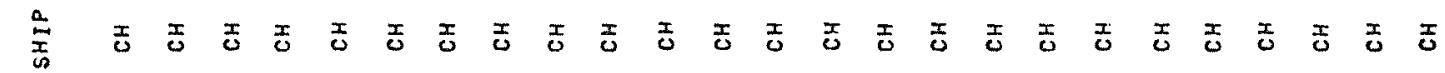

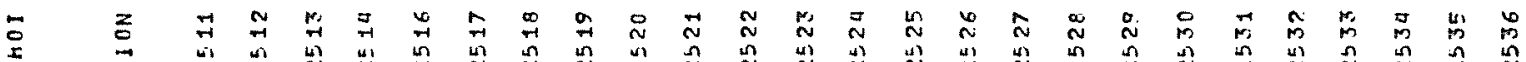

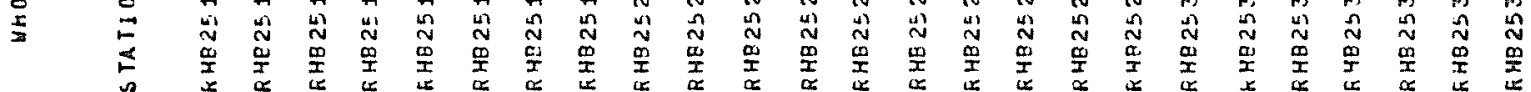




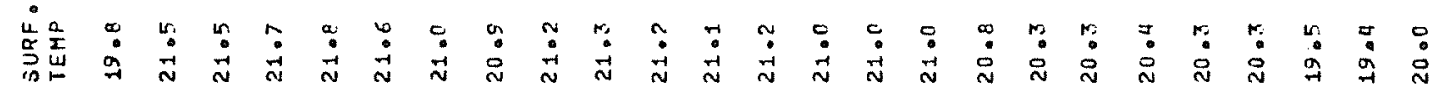

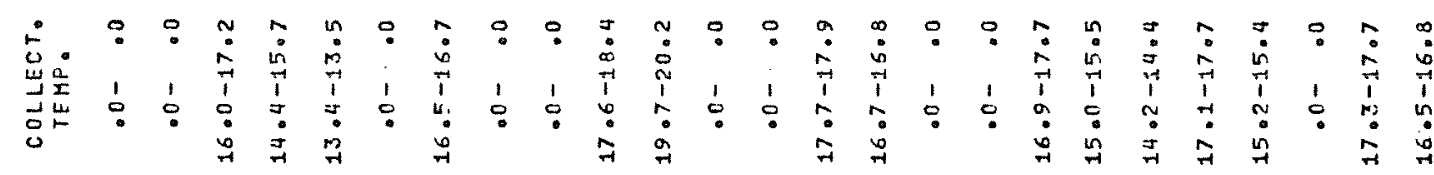

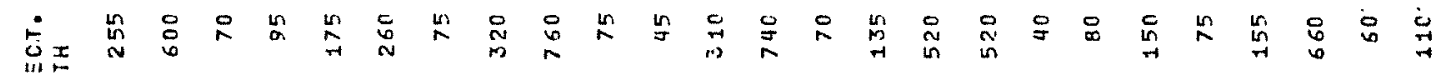

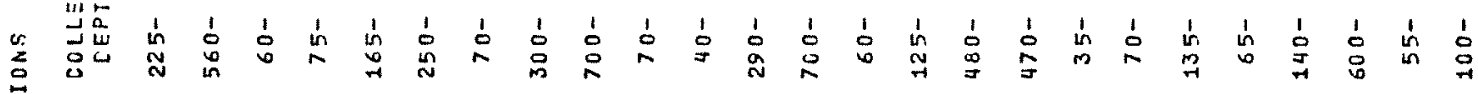

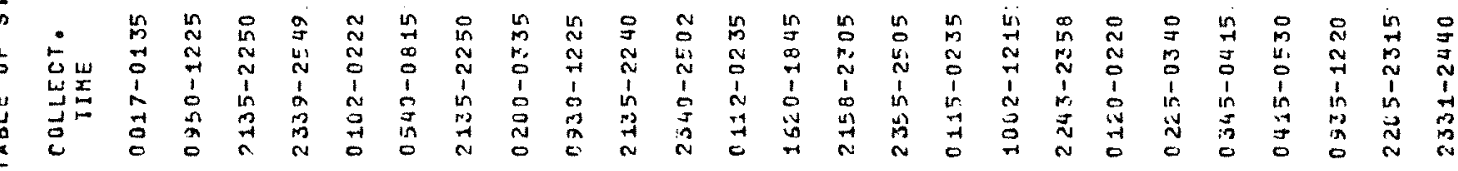

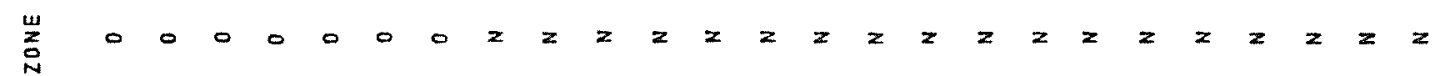

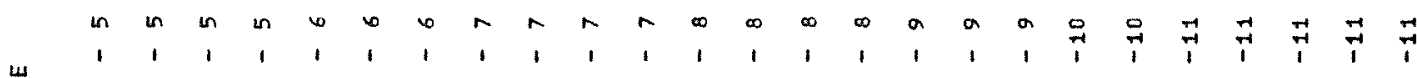

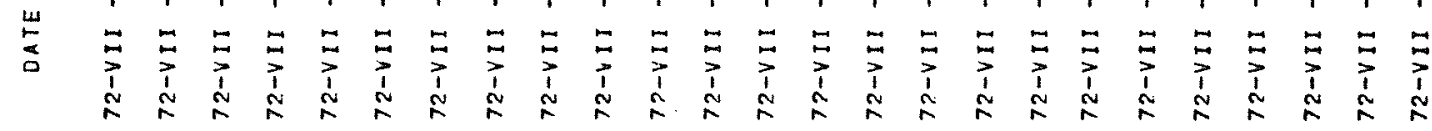

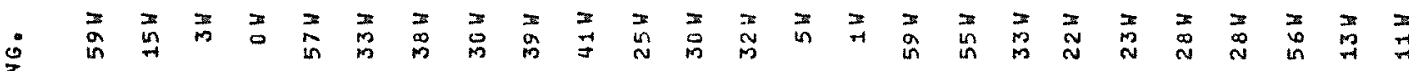

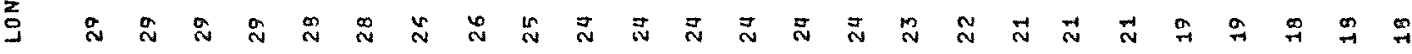

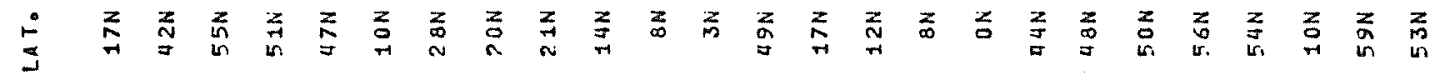

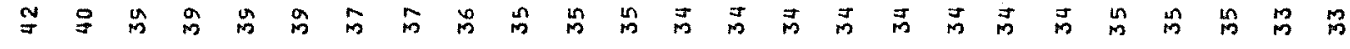

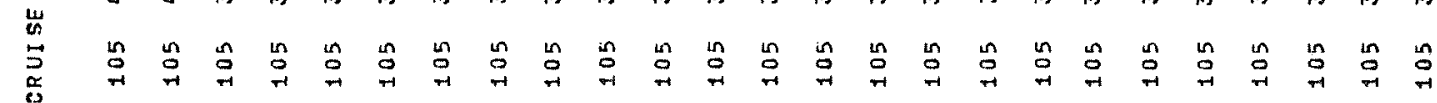

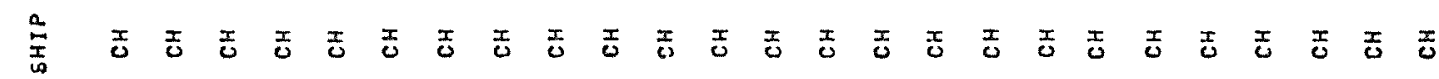

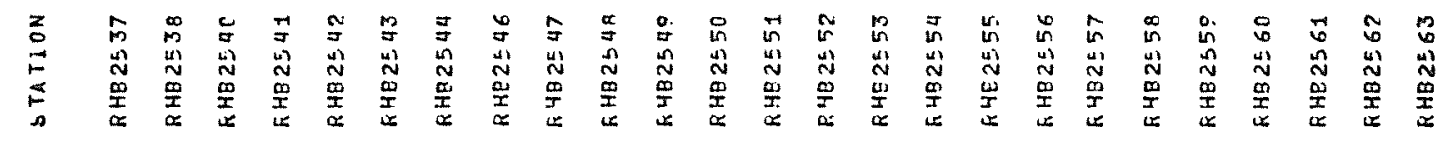




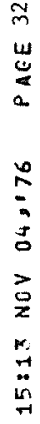

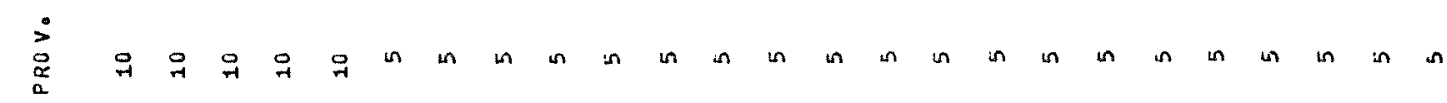

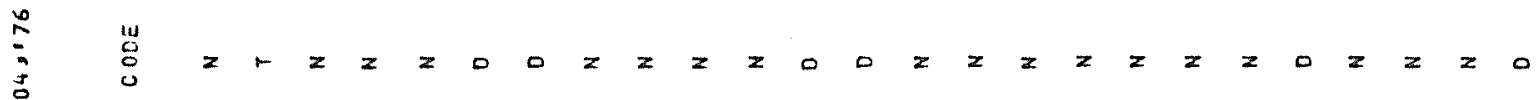

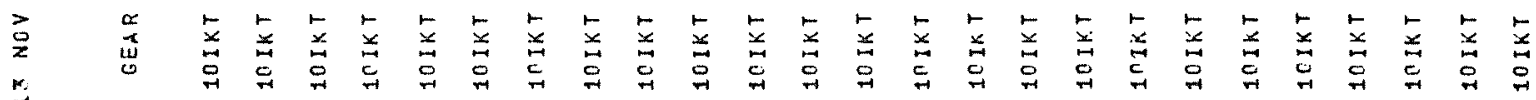

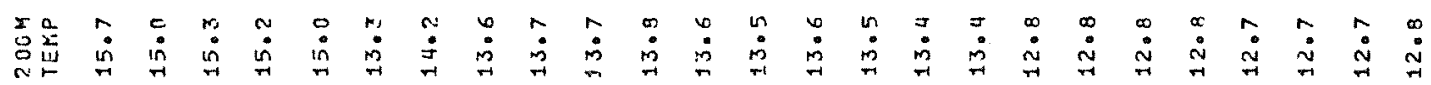

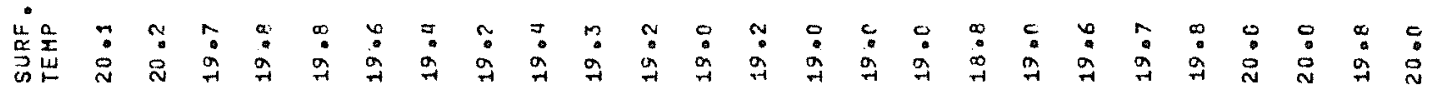

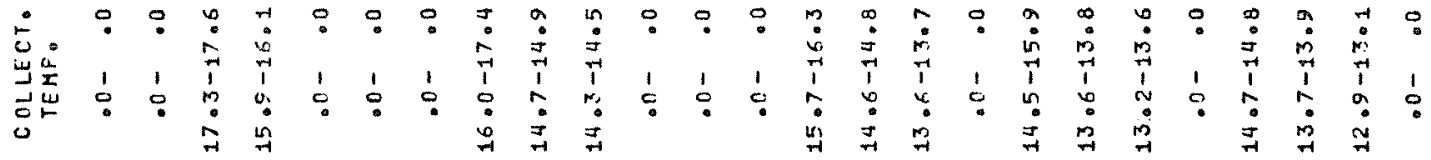

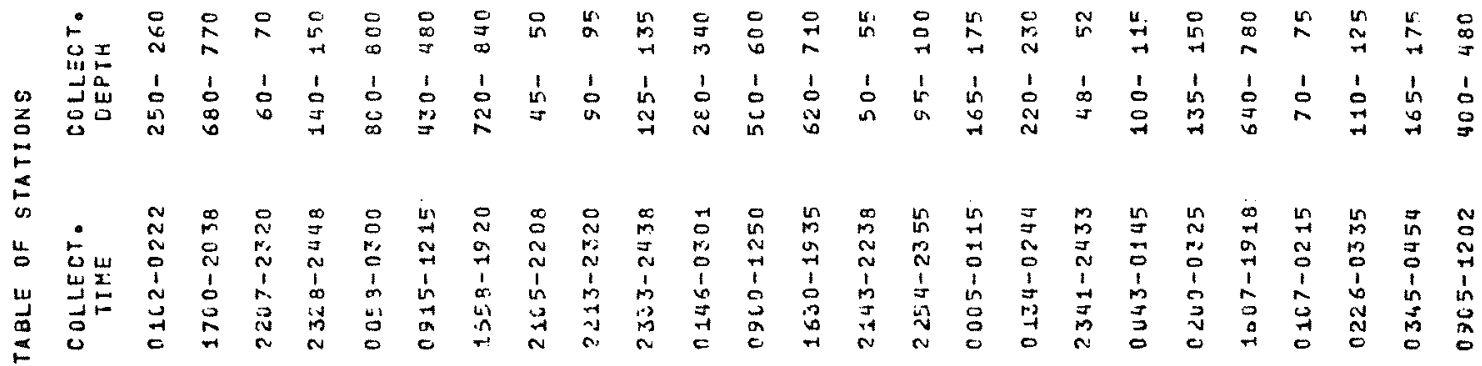

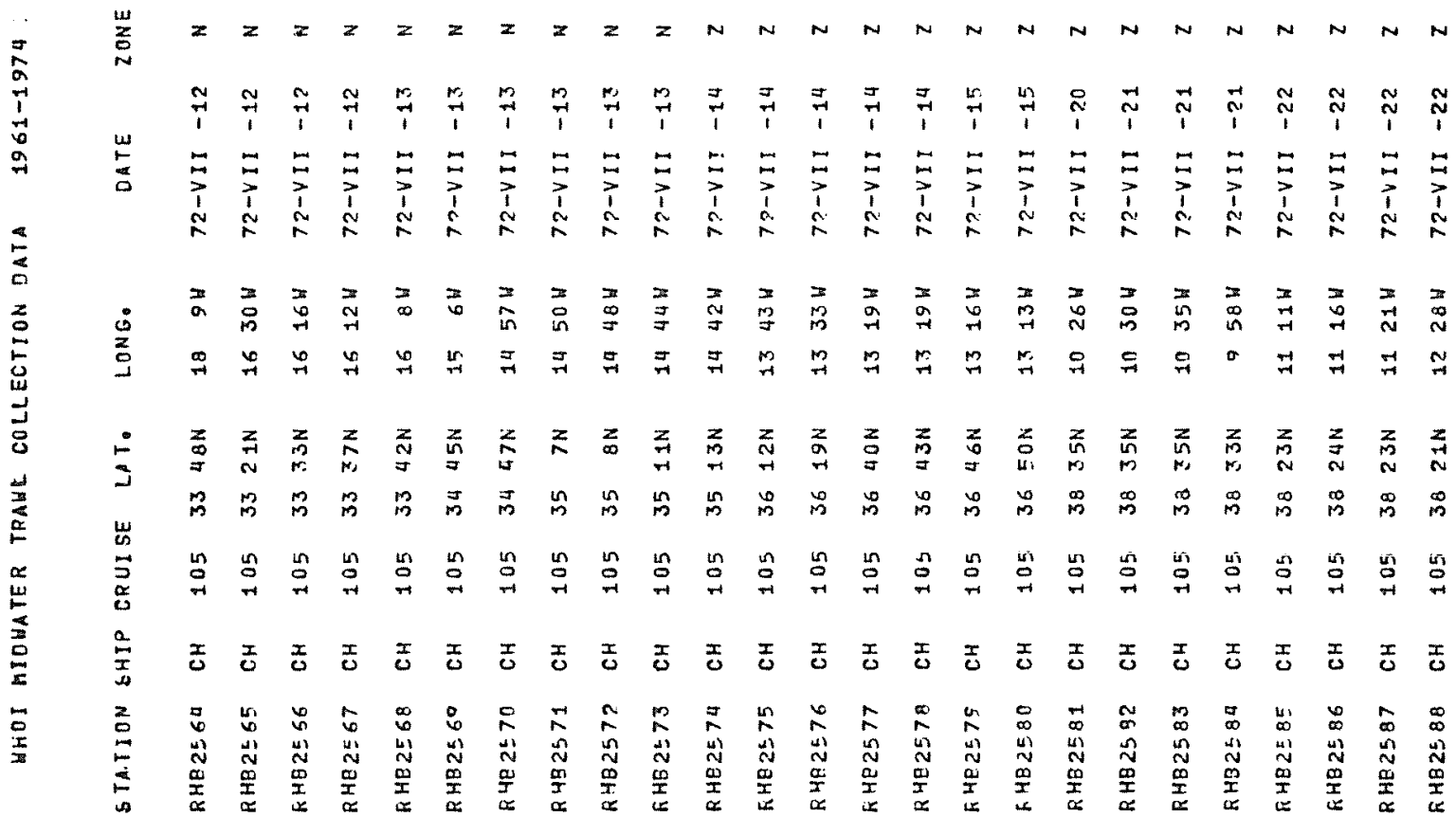


m

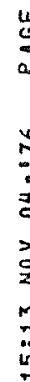

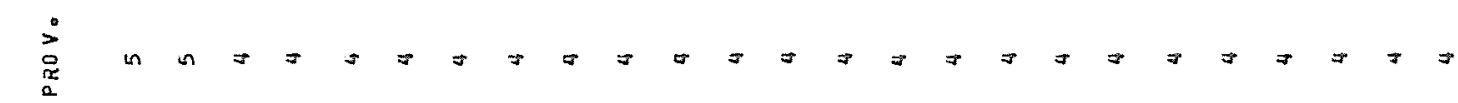
过

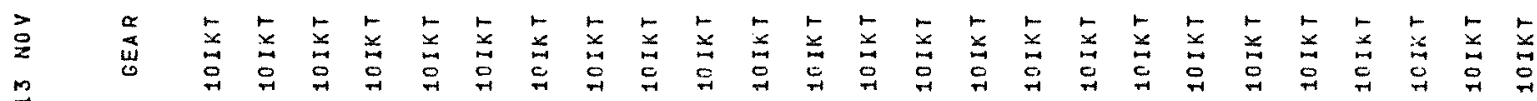
$\stackrel{\ddot{n}}{\rightarrow+1}$

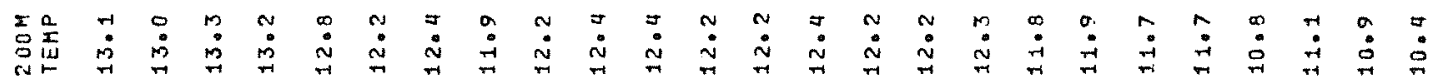

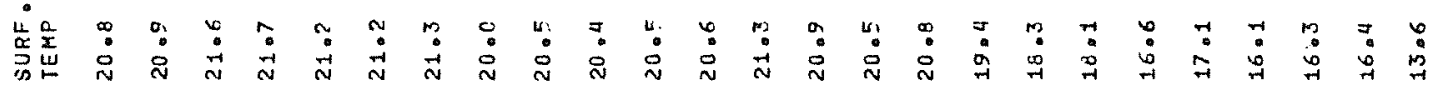

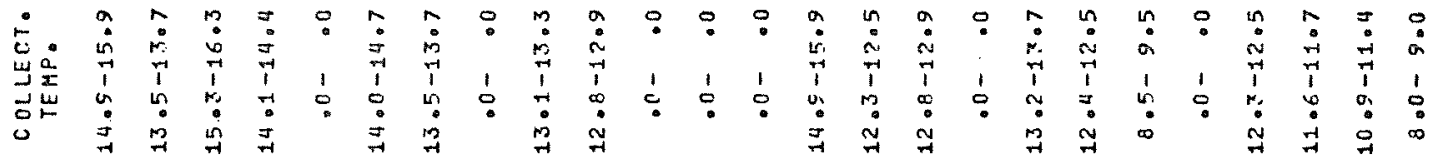

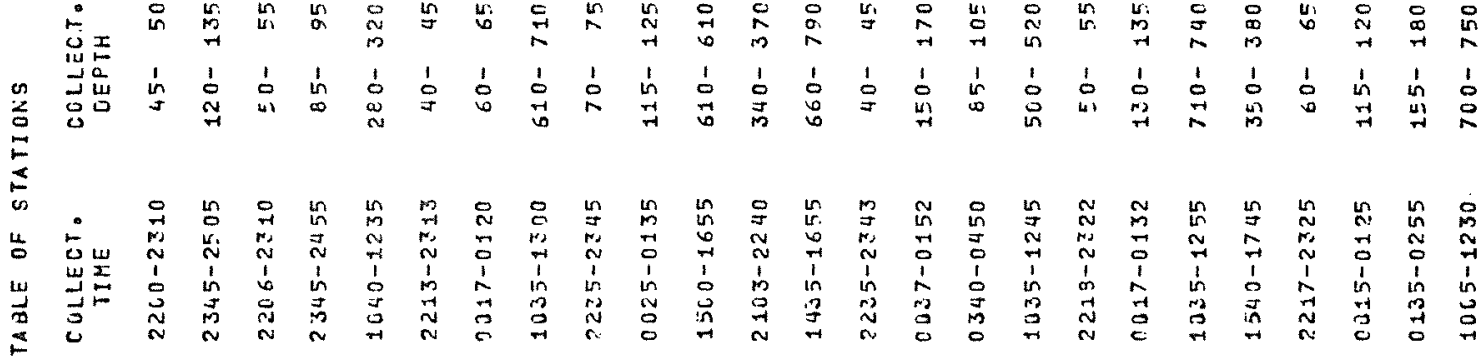

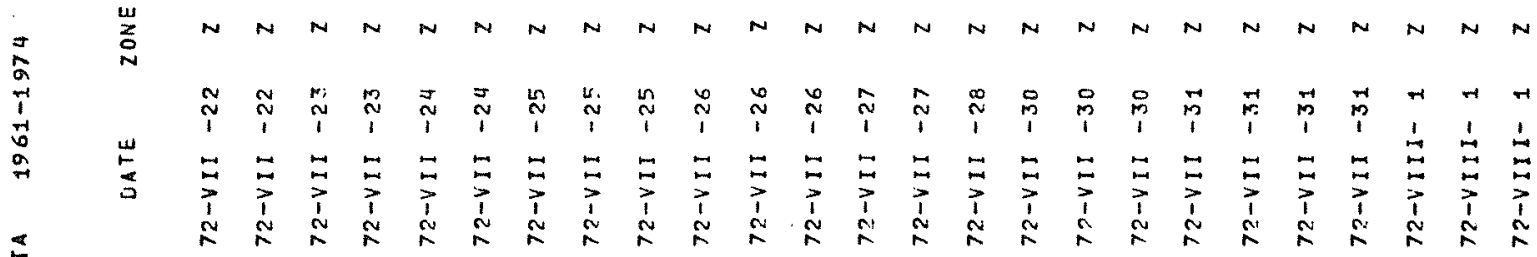

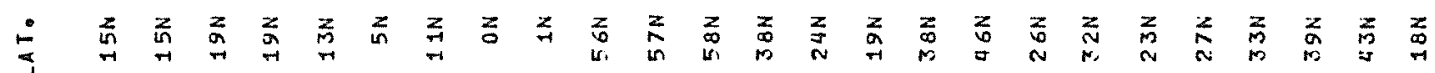

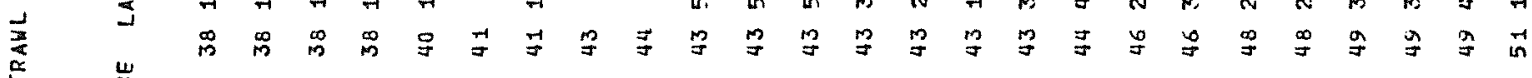

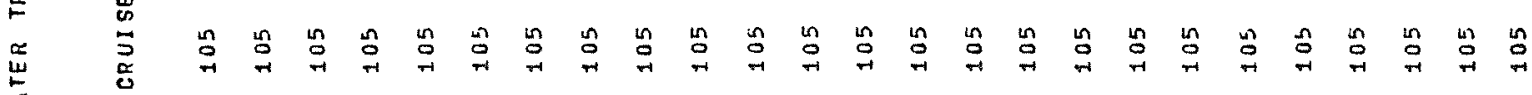
产

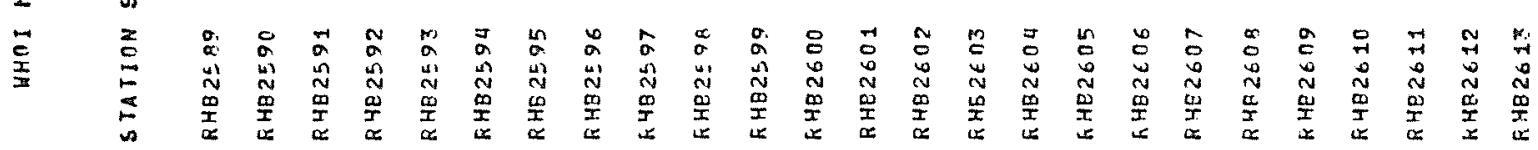


m

$\underset{\substack{w \\ 0 \\ n}}{\substack{0 \\ 0}}$

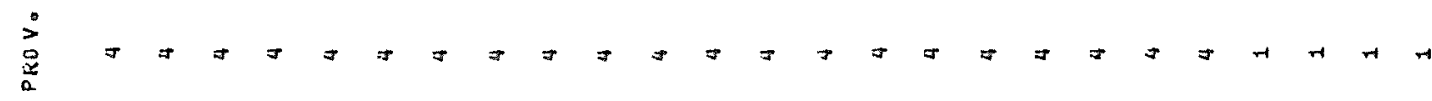

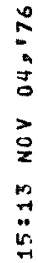

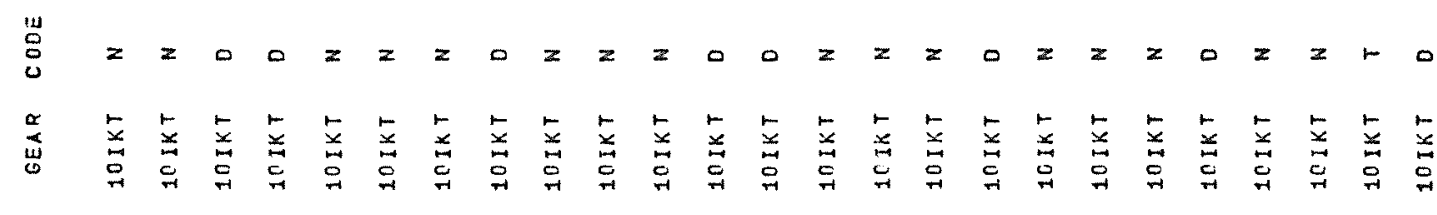

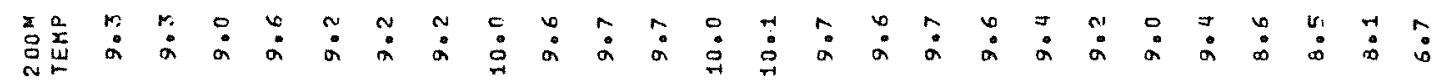

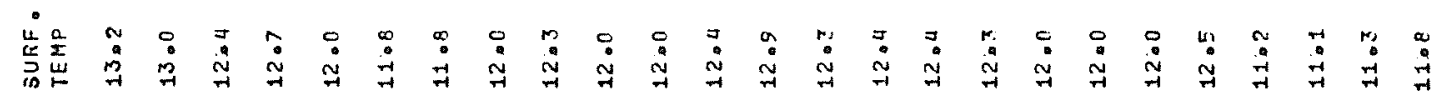

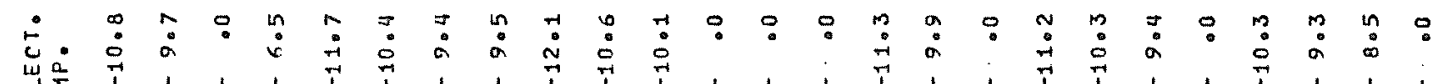

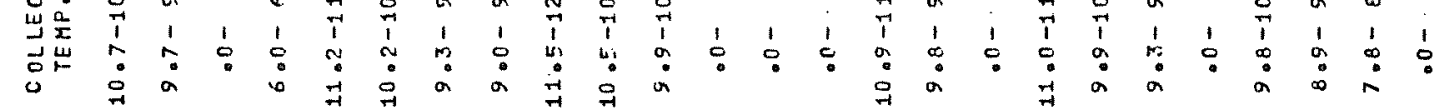

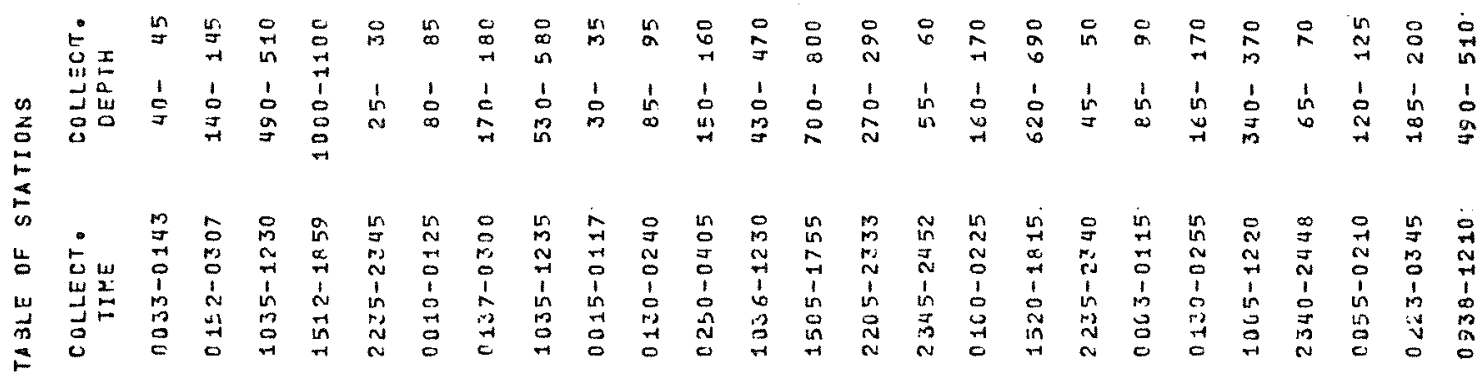

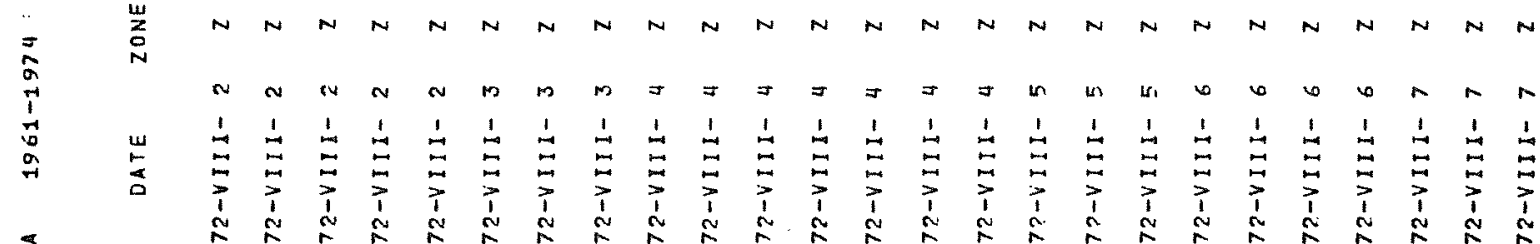

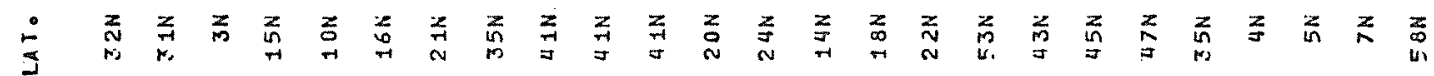
宸

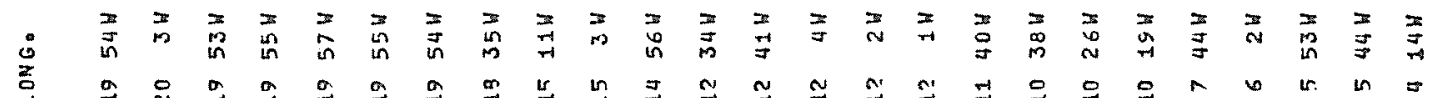

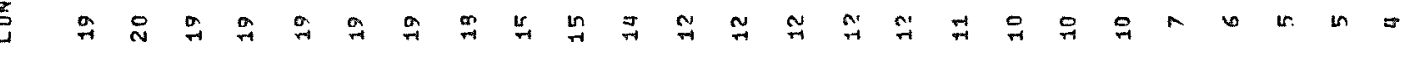

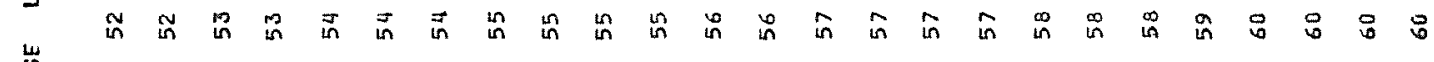

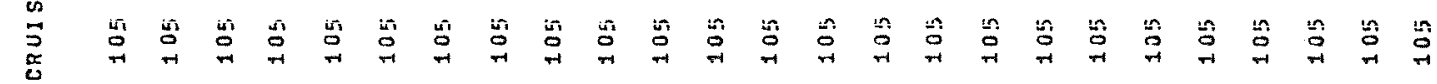

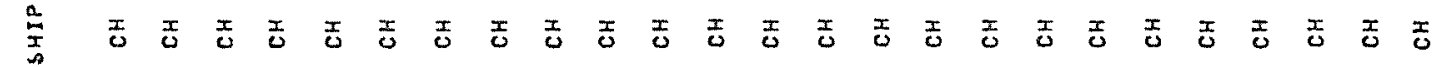

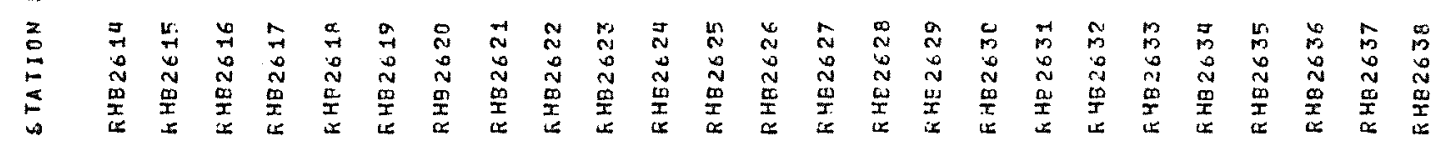


$\stackrel{n}{m}$

$\frac{w}{0}$

$\dot{8}$
$\dot{x}$
0

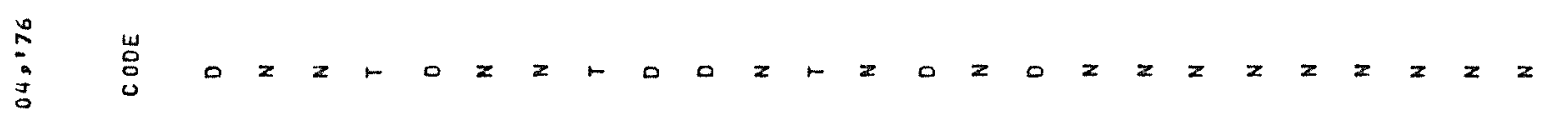

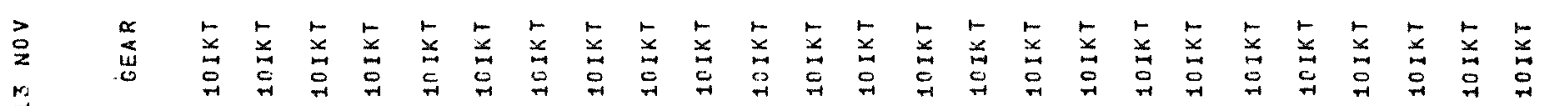
$\underset{\sim}{*}$

至员

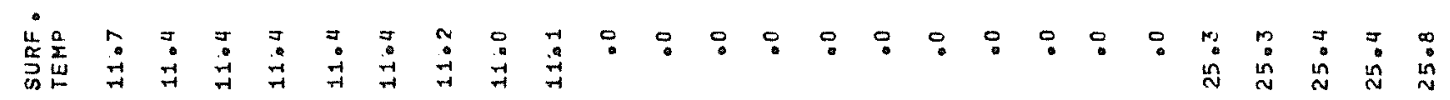

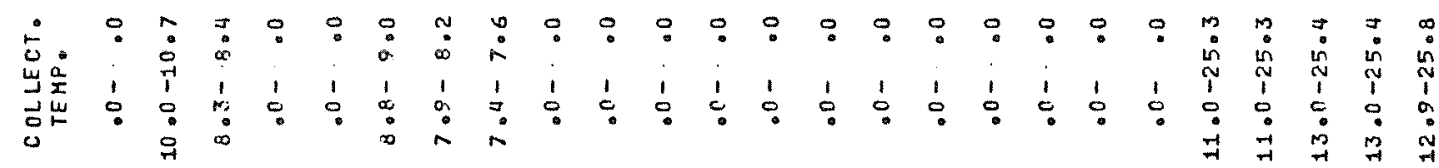

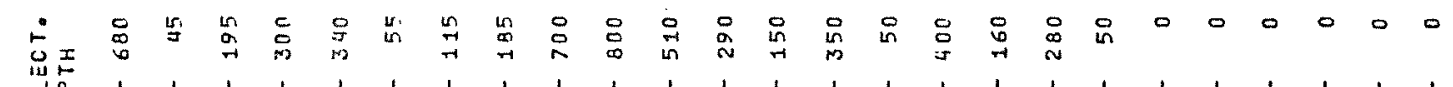

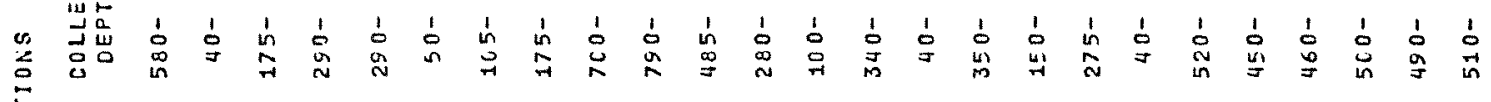

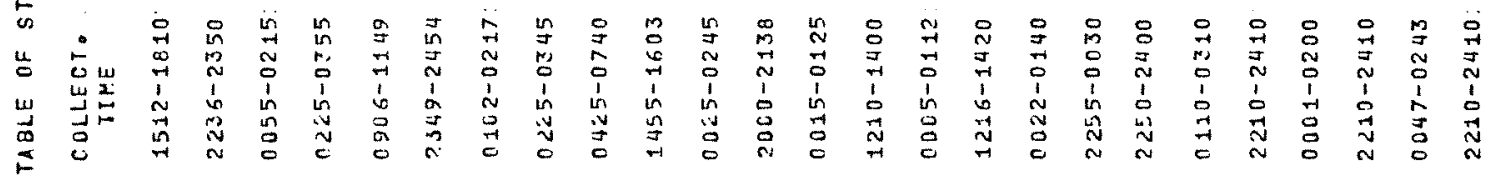

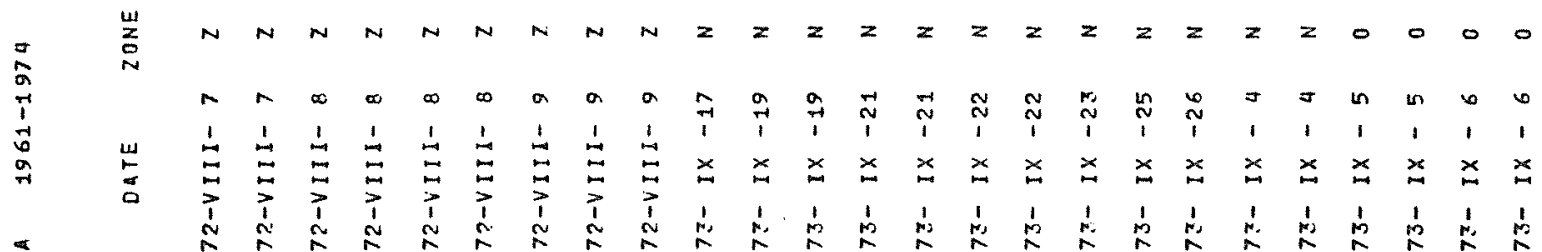

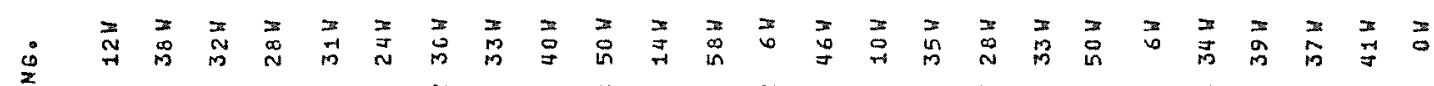

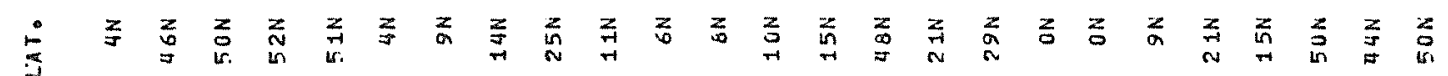

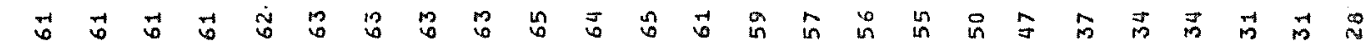

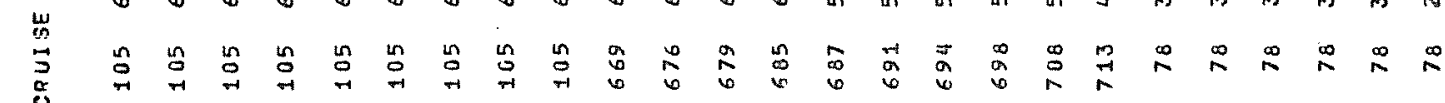

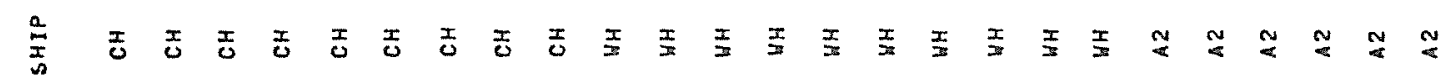

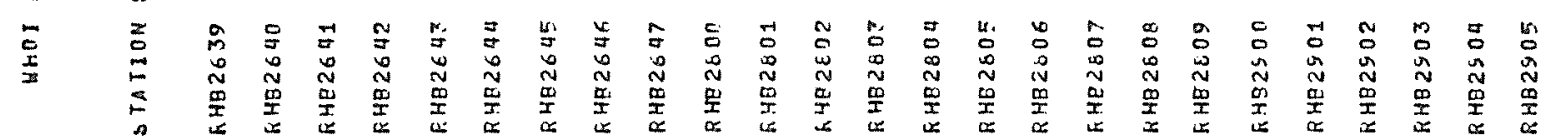


交 总

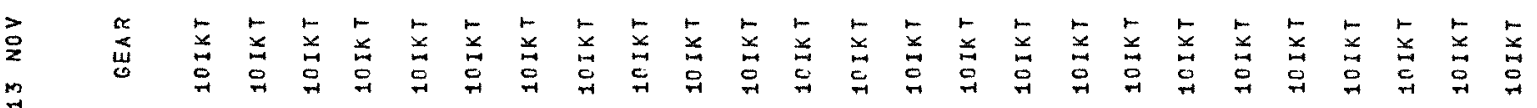

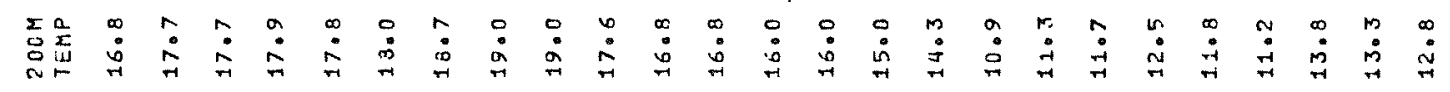
落恙

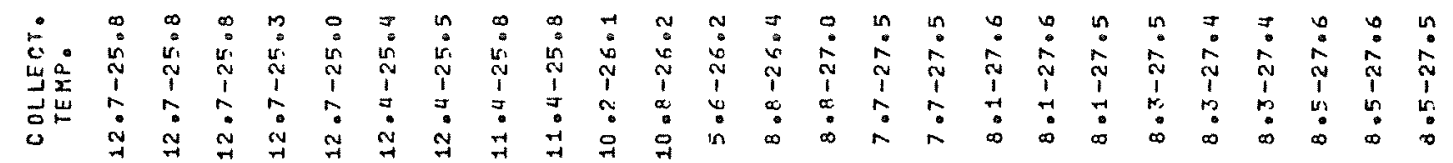

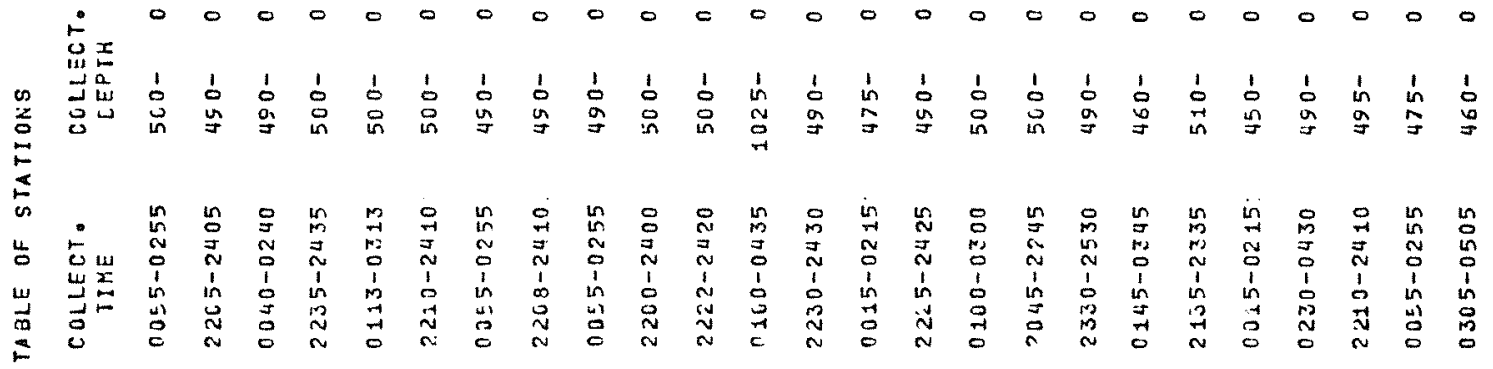

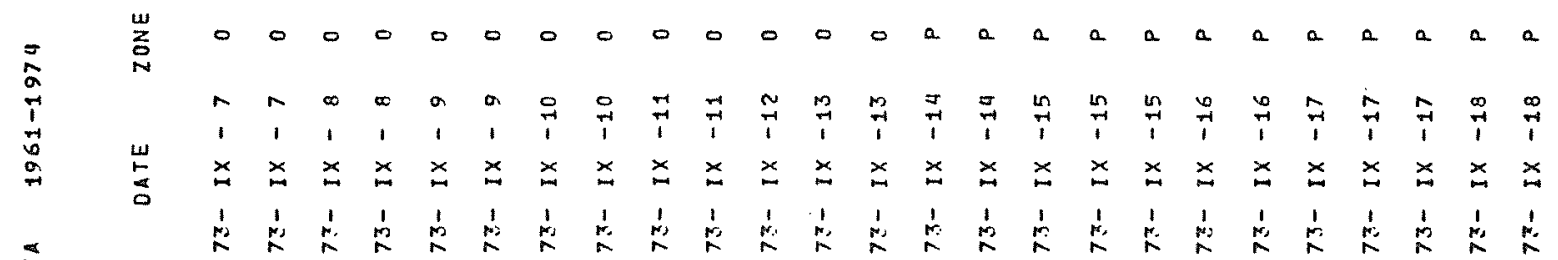

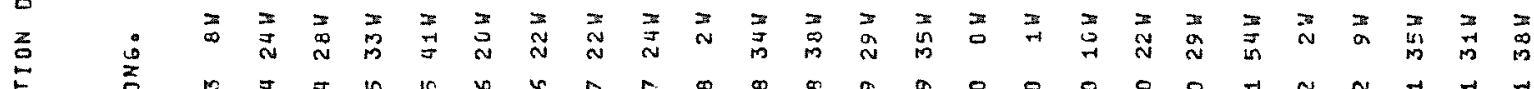

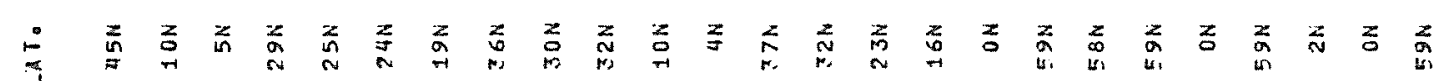
$\stackrel{\infty}{N} \hat{N} N \stackrel{N}{N} \stackrel{N}{N}$ i 崖 焉

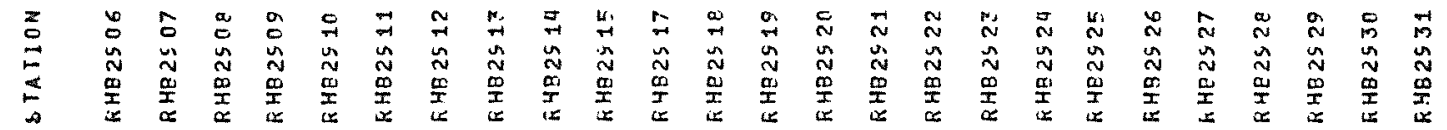




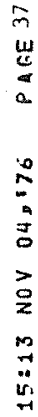

$\dot{\vec{c}}$
0
0 :

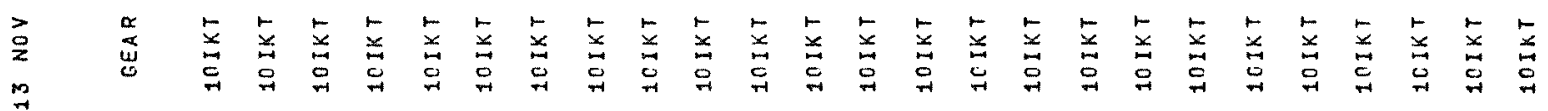

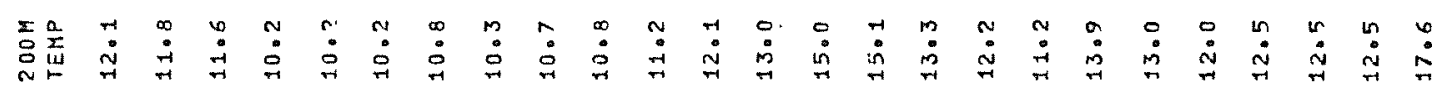

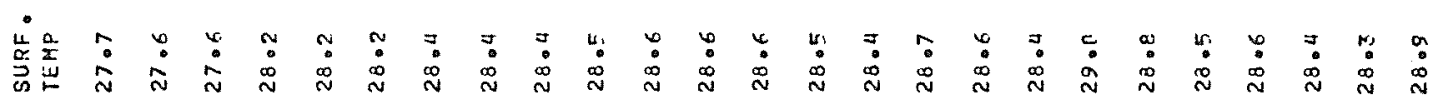

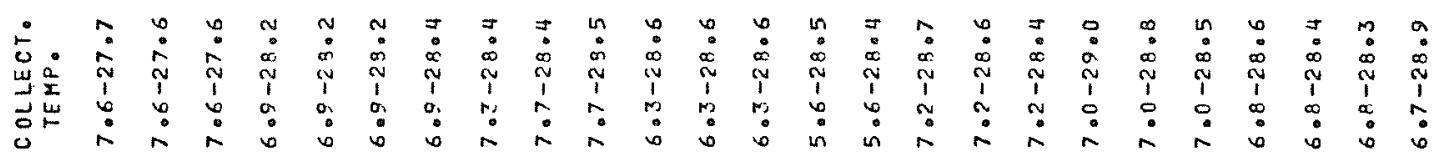

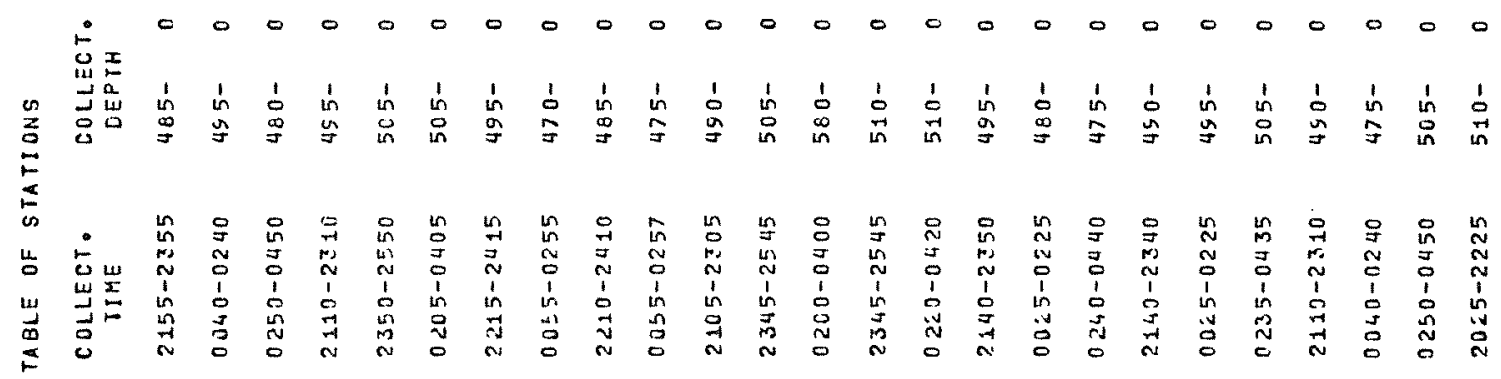

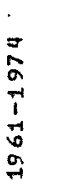
崖 a a a a a a a a a a a a a a a a a a a a a a a a a

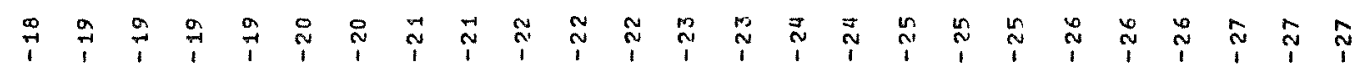

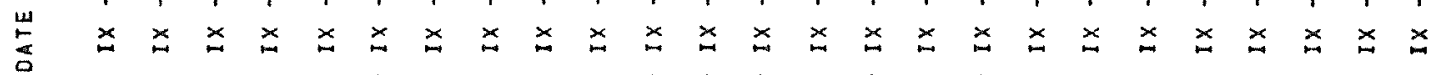

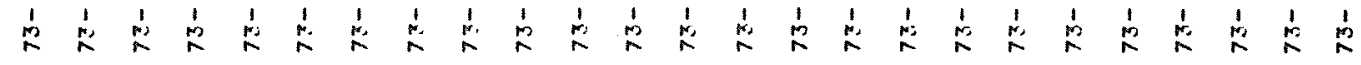

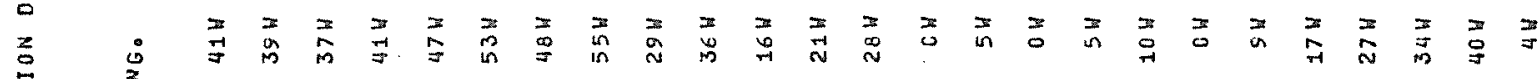

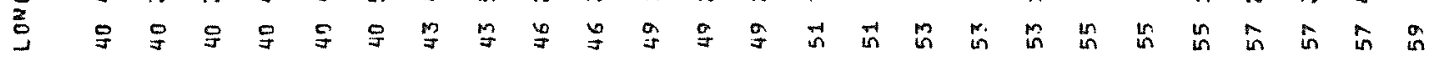

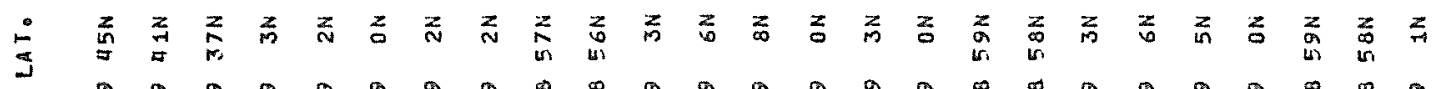

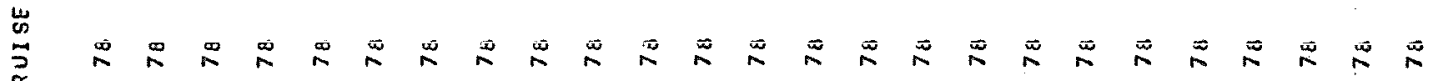

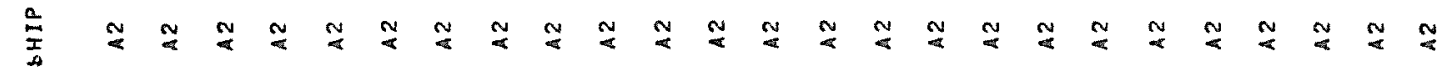

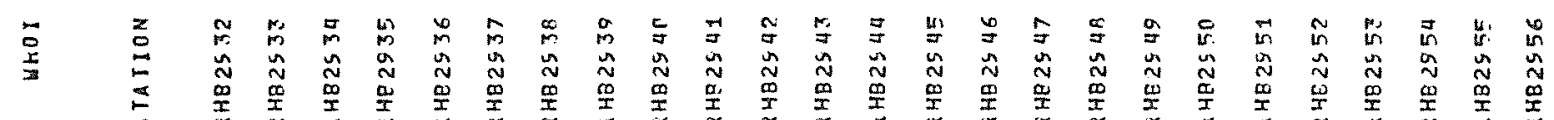




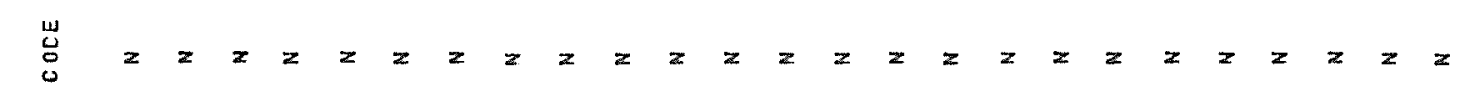

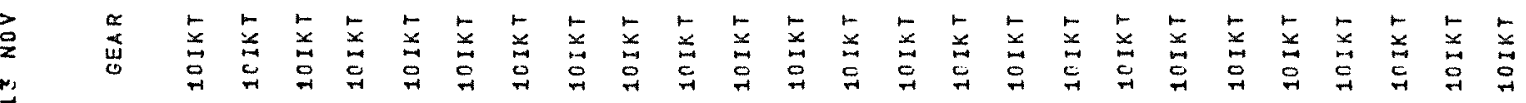
宏

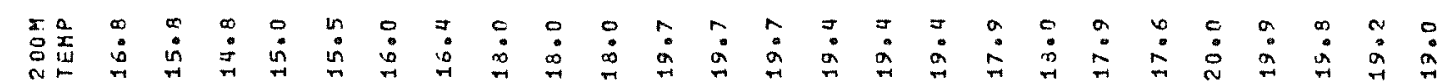

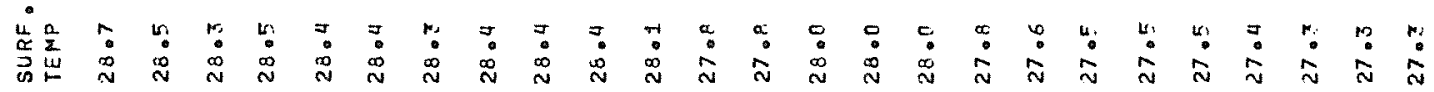

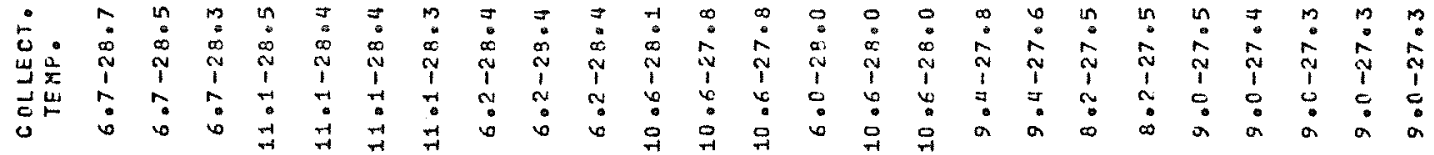

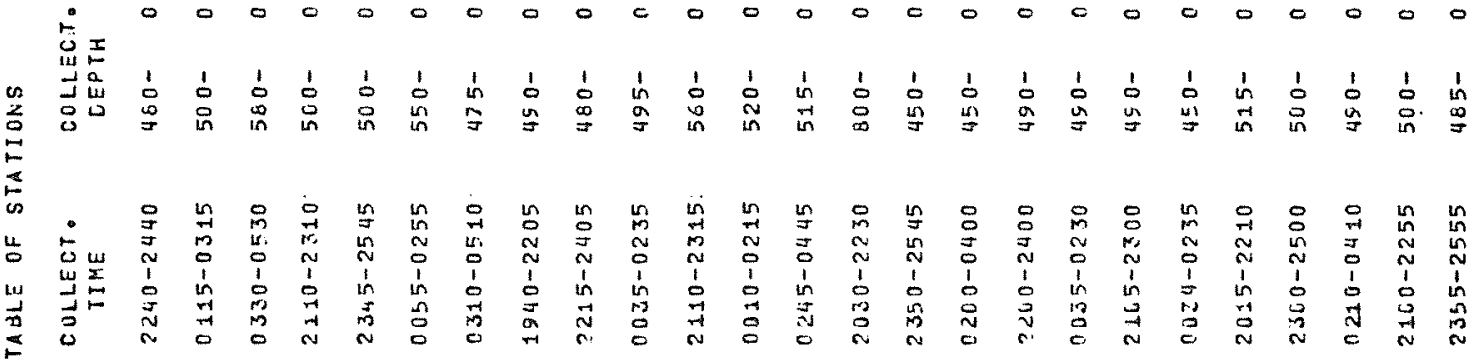

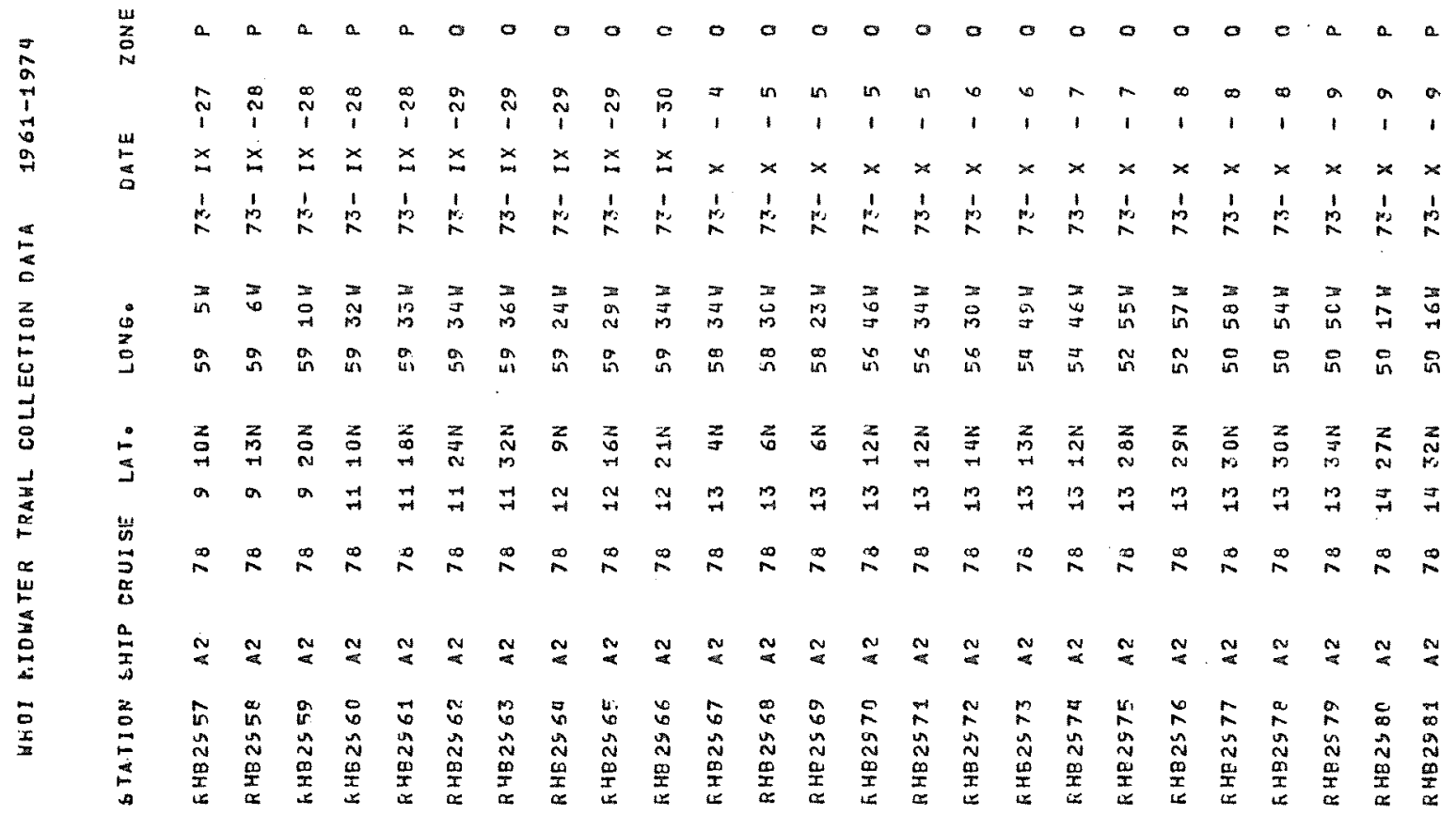




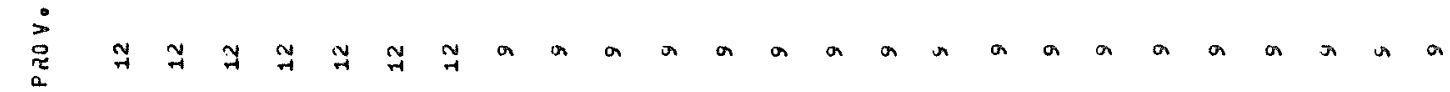

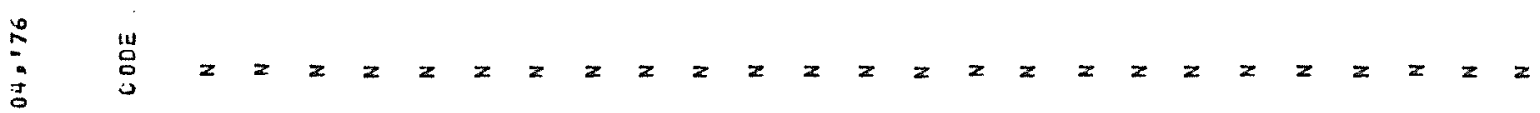

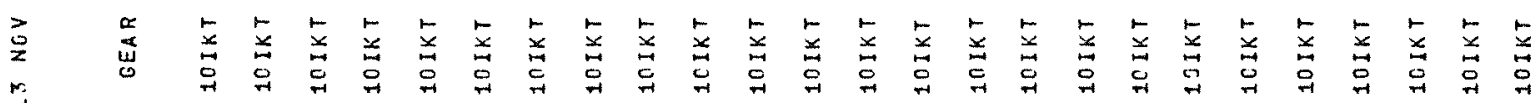

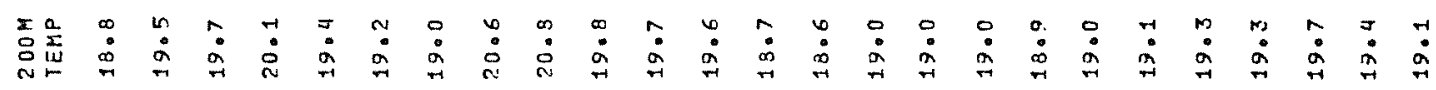

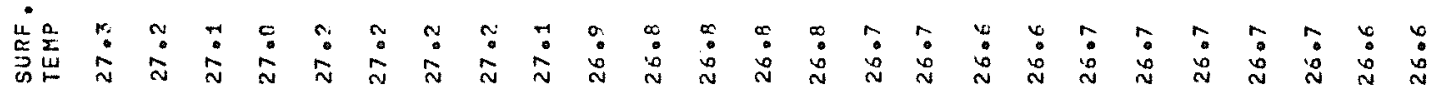

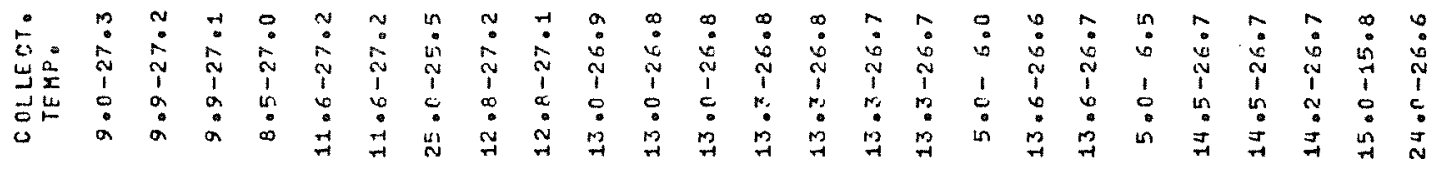

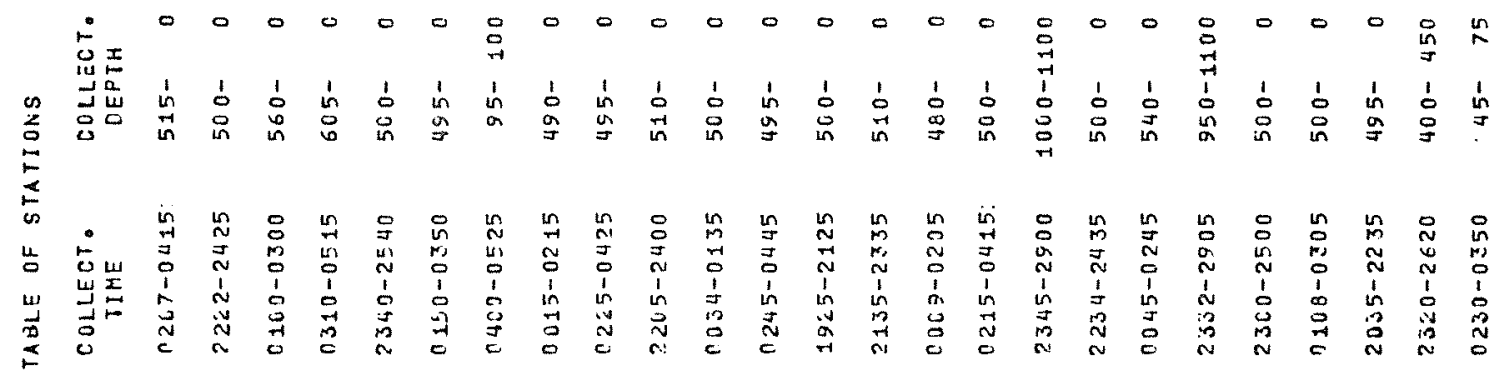

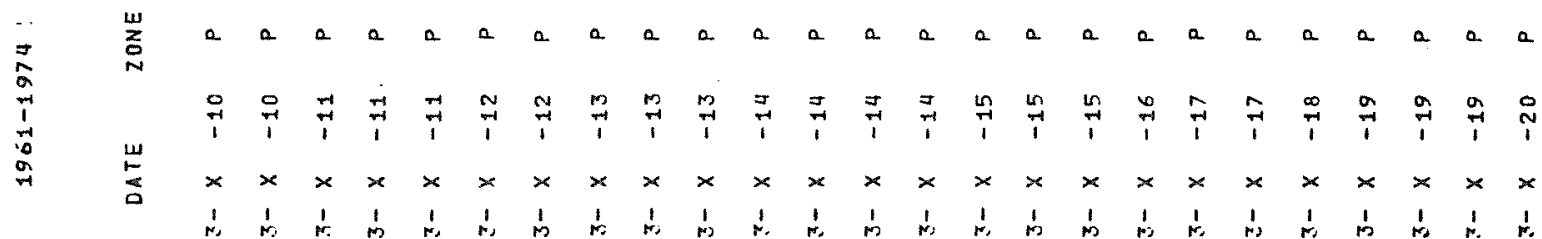

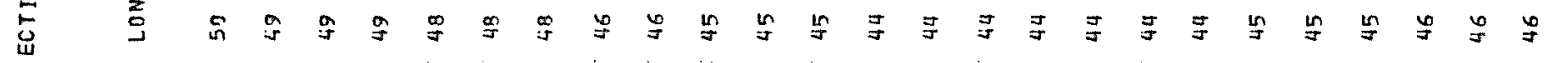

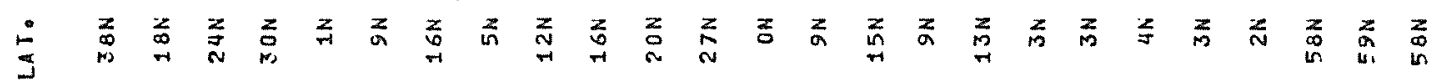

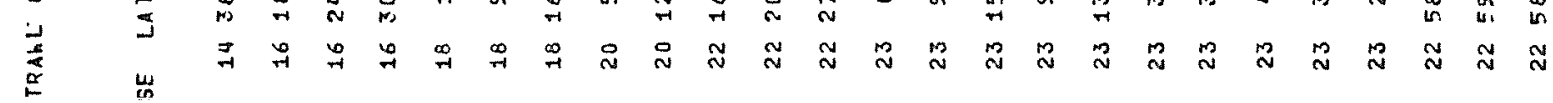

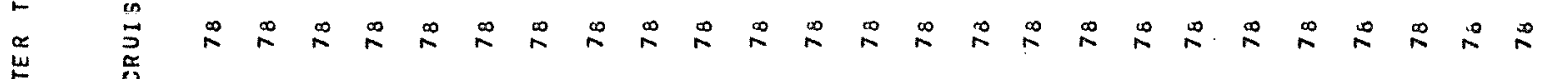

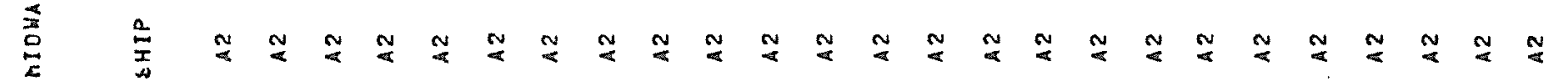

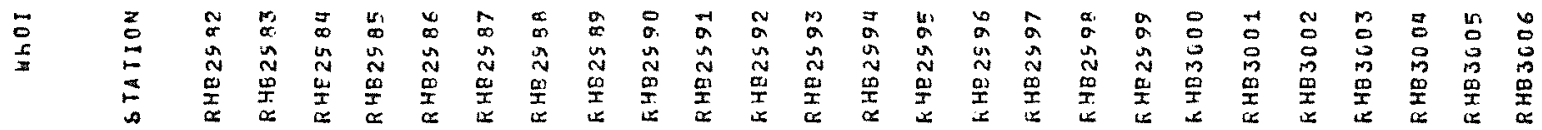


8
$\stackrel{0}{u}$
0
$a$
0

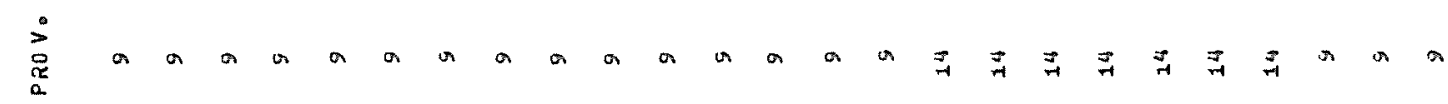

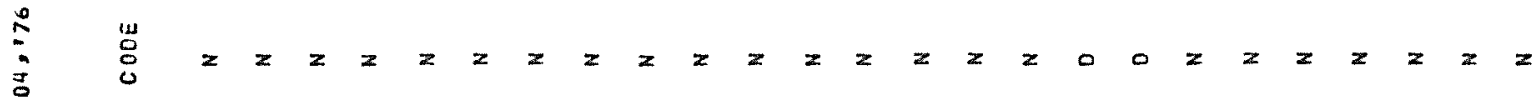

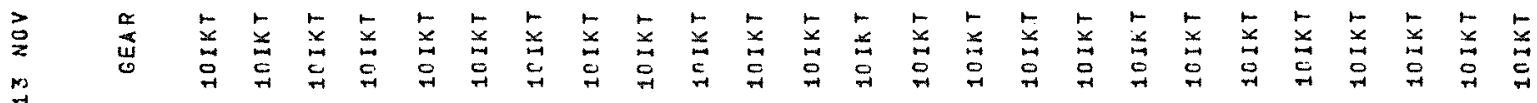

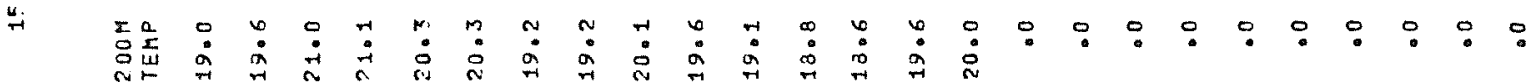

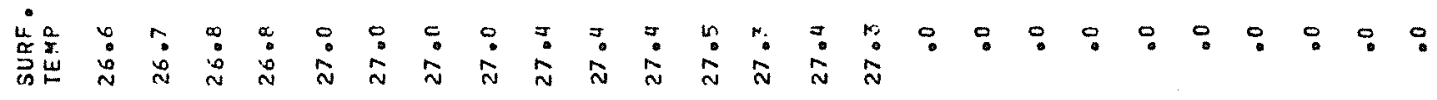

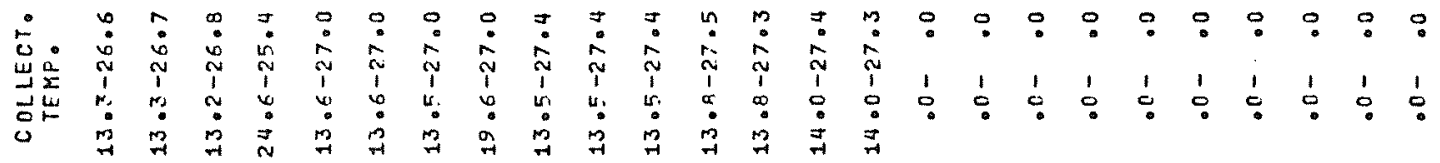

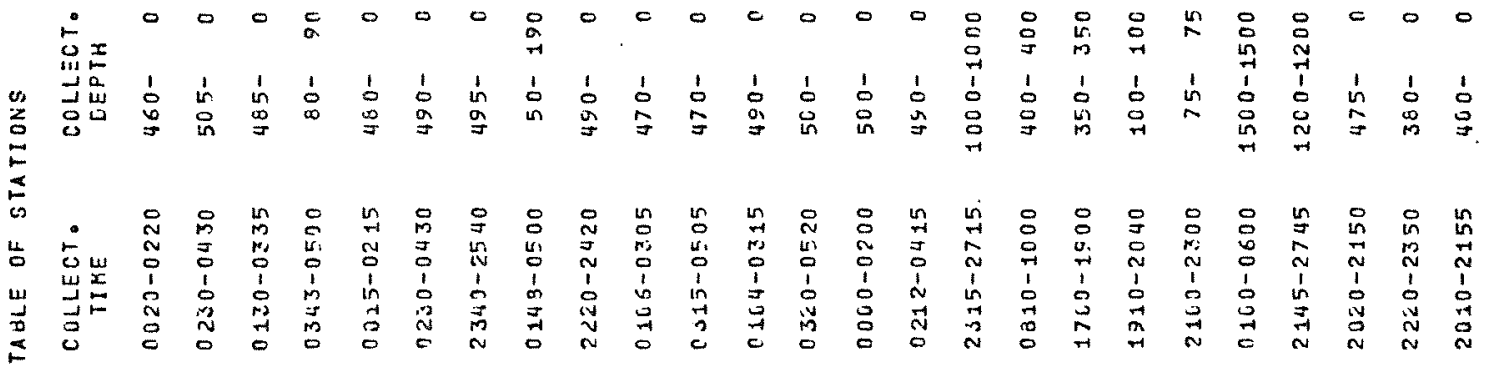
苦

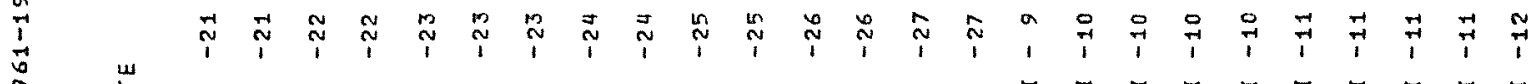

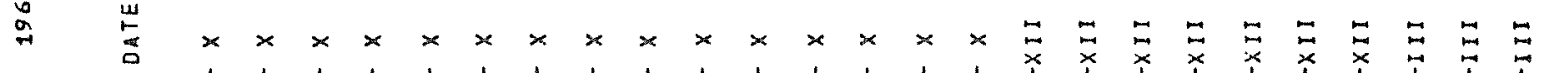

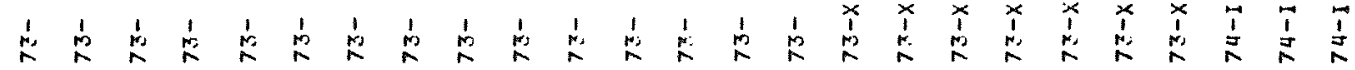

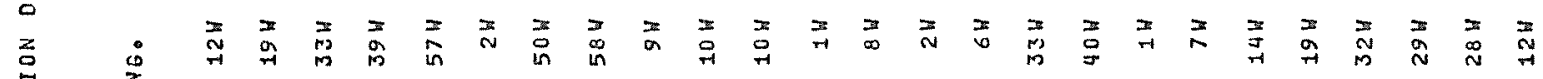

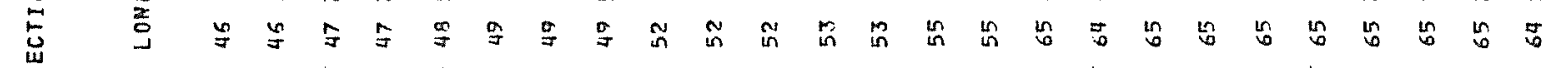

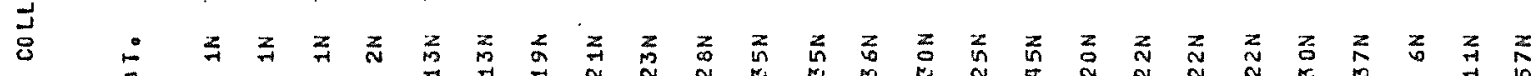

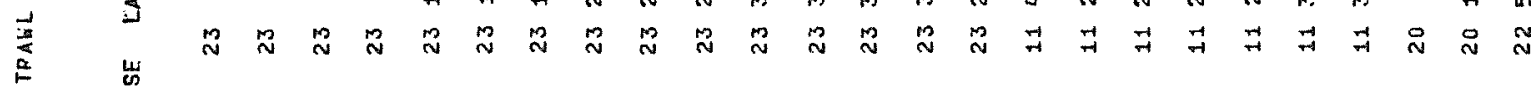

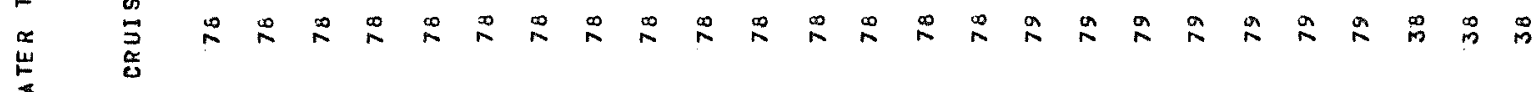

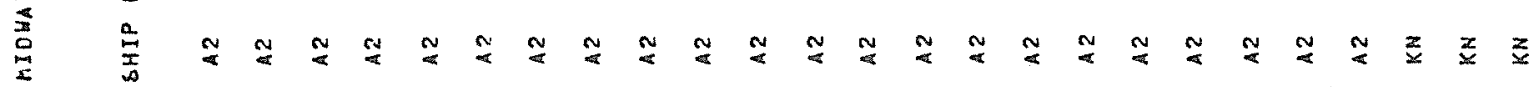

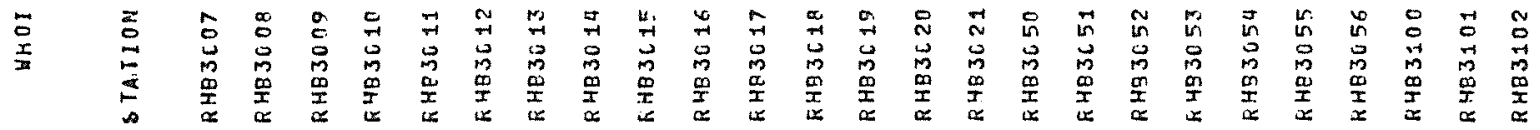


$\exists$

ü

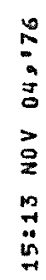

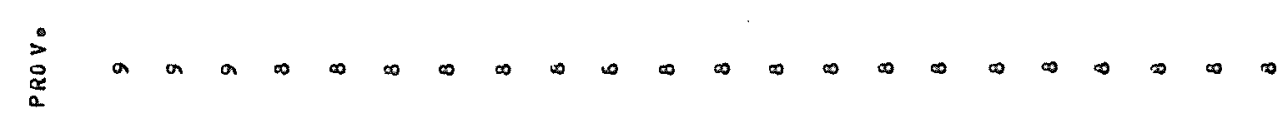
:

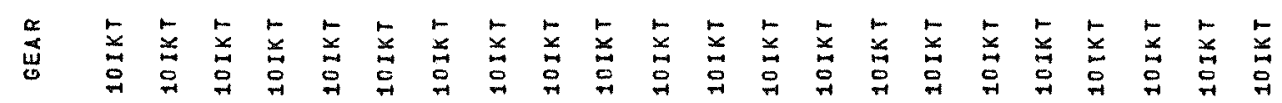

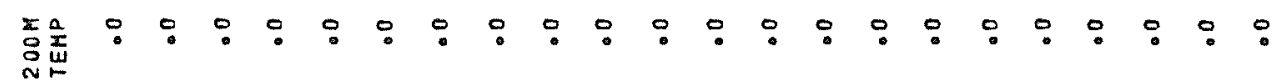

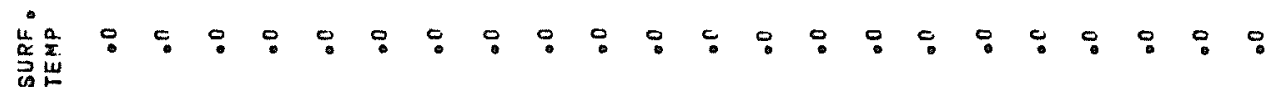

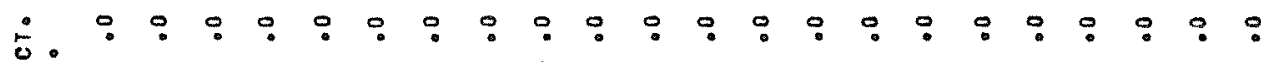

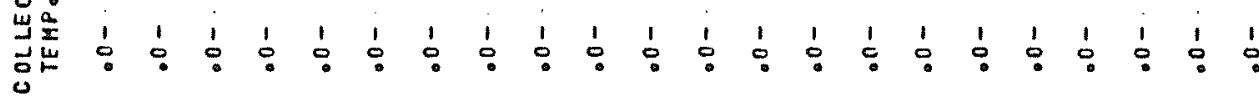

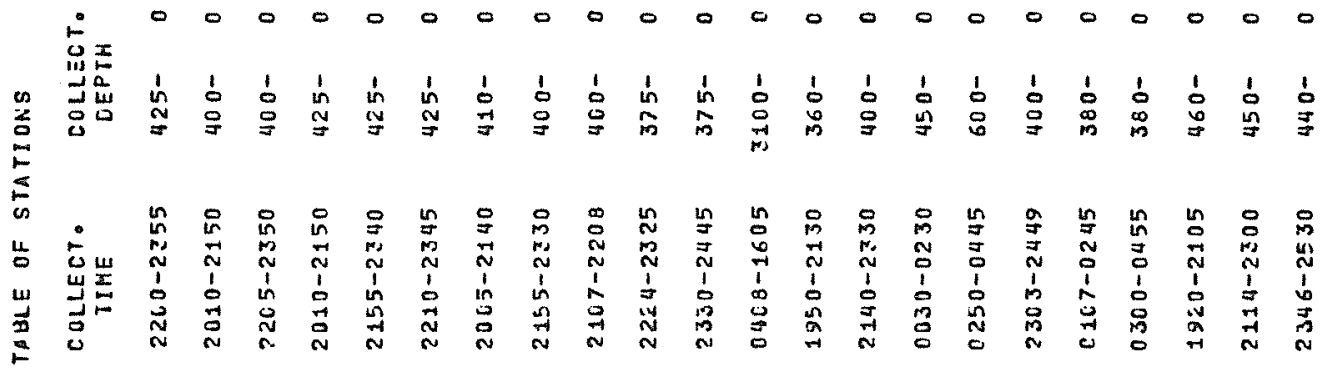

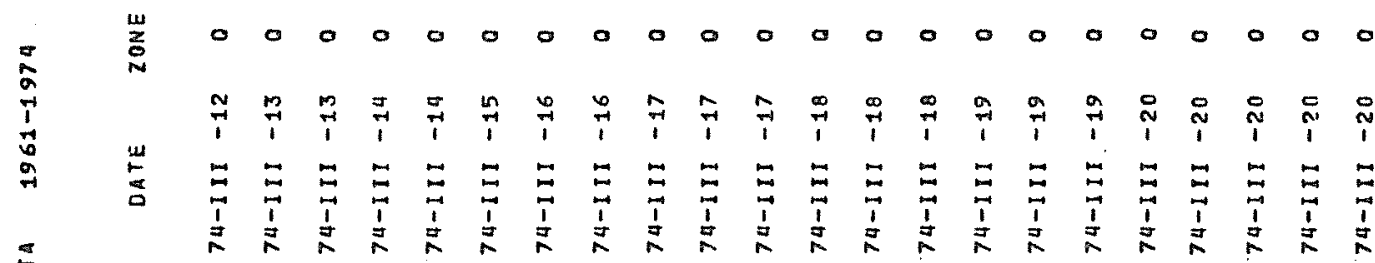

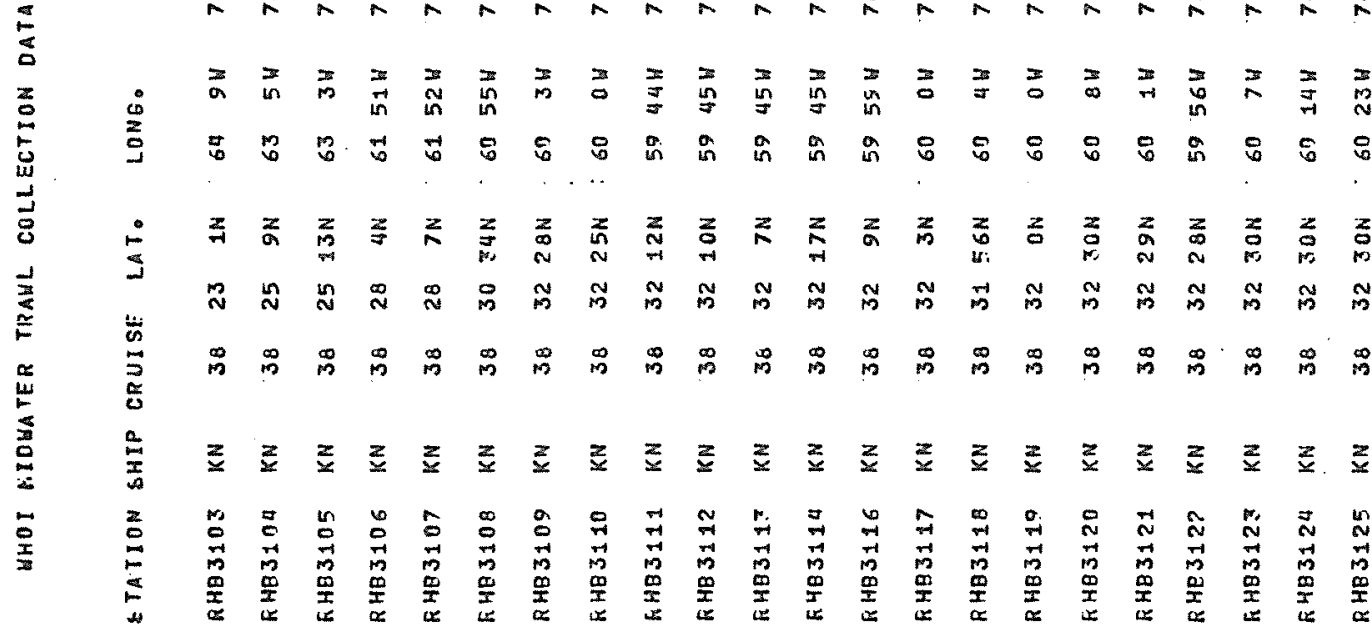




$$
\text { II-1 }
$$

Appendix 2

WHOI neuston Collection Data, 1964-1974.

Most of the neuston collections (SUN 1003-3114) were made simultaneousIy with midwater trawls; the collection numbers correspond (i.e., SUN 1858 accompanied RHB 1858). Collections SUN 9200-9904 did not accompany midwater trawls.

Al1 neuston collections were made at night. Often two, three, or four nets were fished simultaneously. When more than one neuston haul was made during a single midwater trawl (or at one position for those not accompanying midwater trawls), the 'Collection Time' is artificial; it is, in such instances, simply adjusted to equal the pooled durations of the individual hauls made during a single midwater trawl (or at a single position).

For further explanation, see Appendix 1. In addition to the ships in Appendix 1, LU = Lulu, and $\mathrm{CR}=$ Crawford. 
II-2

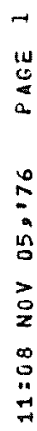

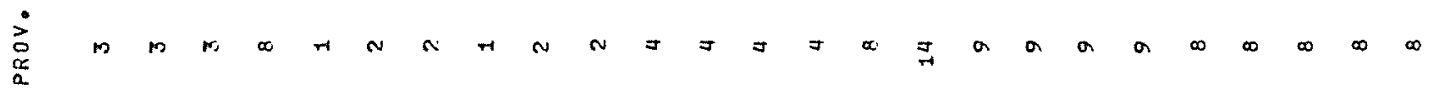
0
0

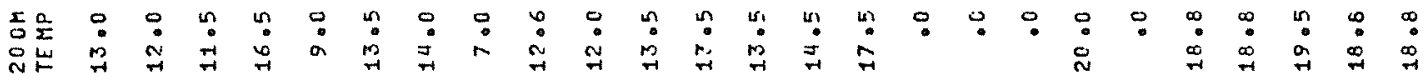

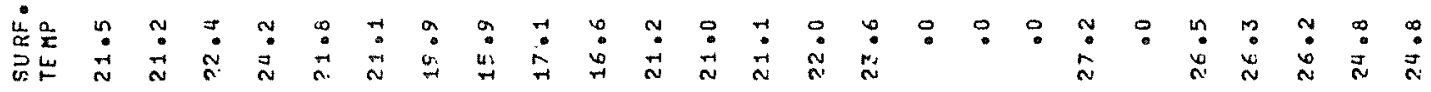

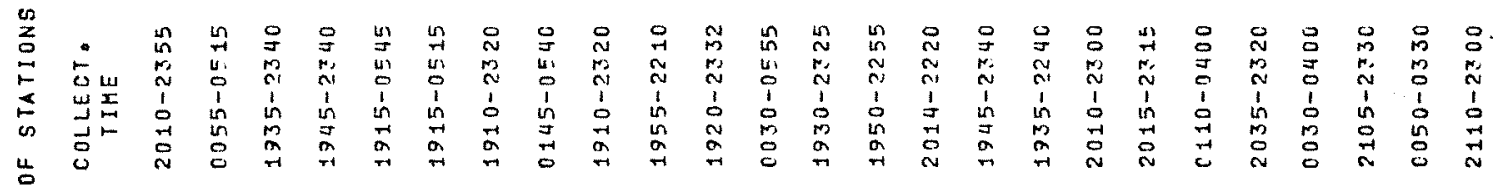

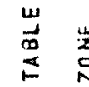

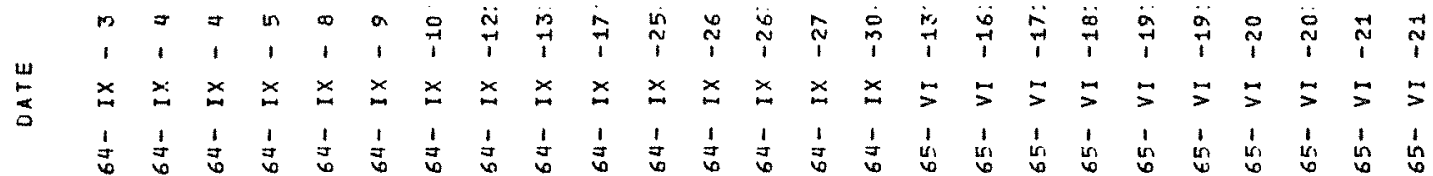

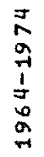

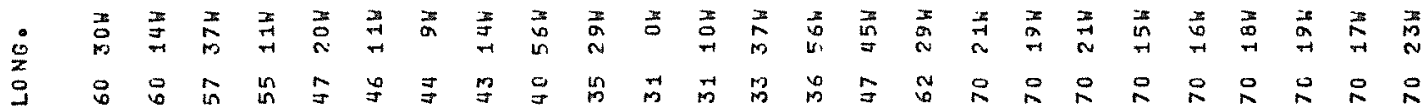

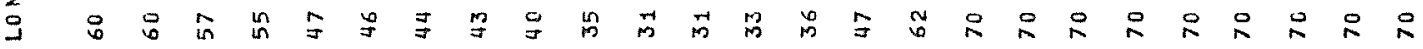

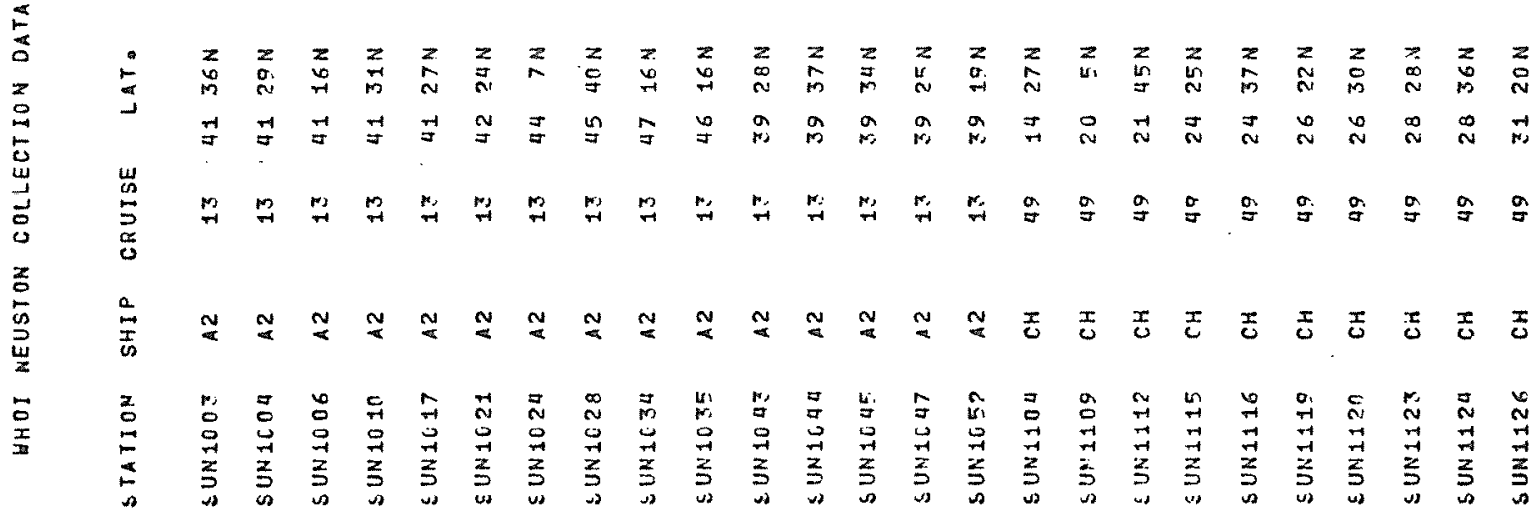


$n$
$u$
0
0
$a$
0
0
0
0
0
0
2
0
0
0
0

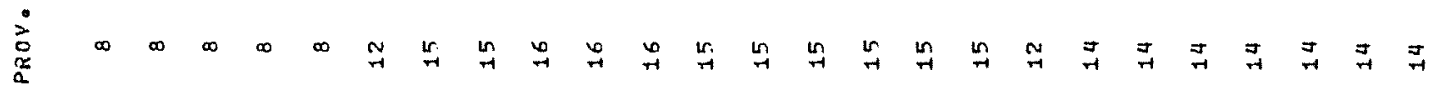

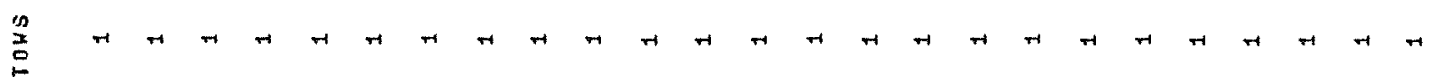

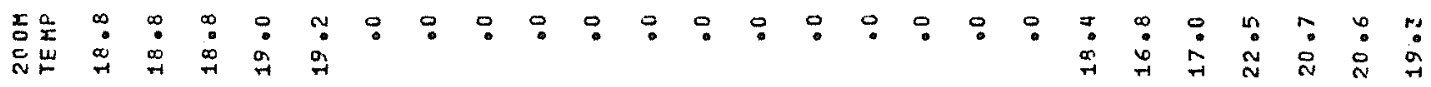

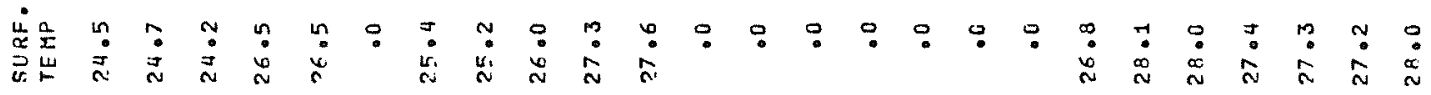

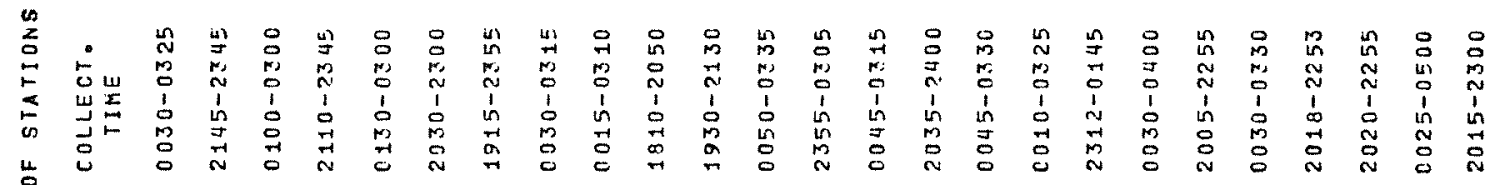
$\stackrel{m}{\vec{x}}$

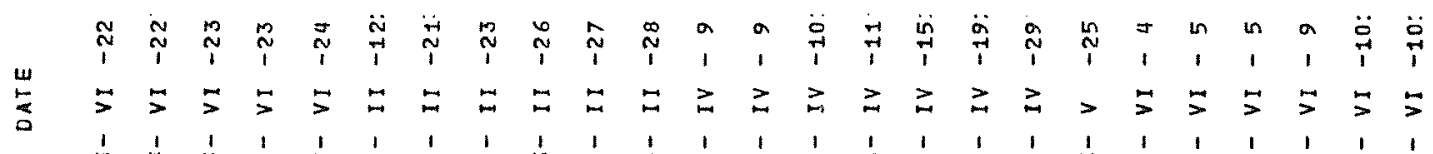

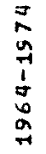

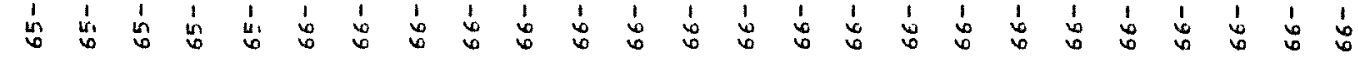

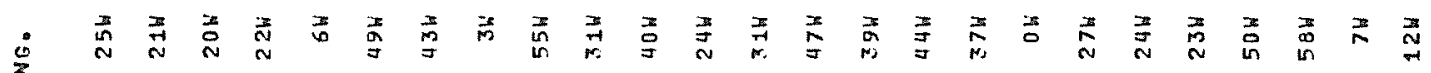

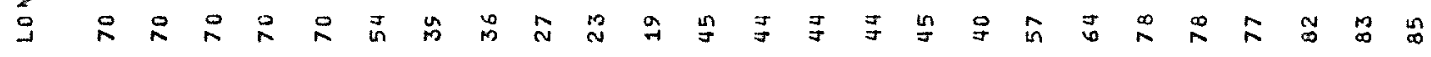

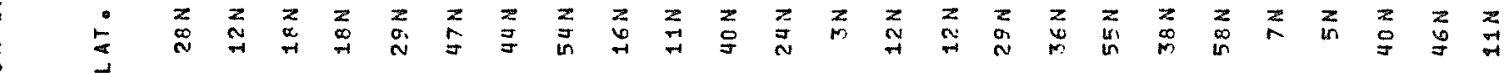

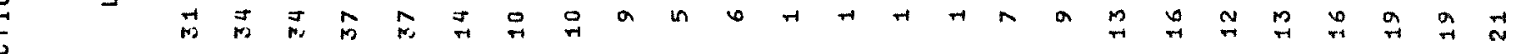

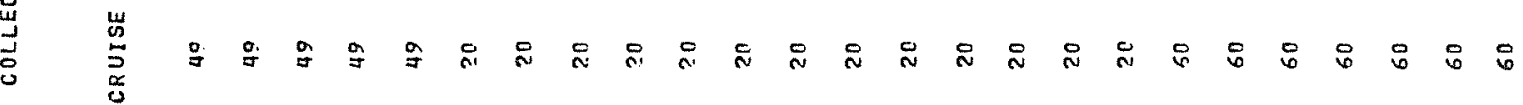

焉

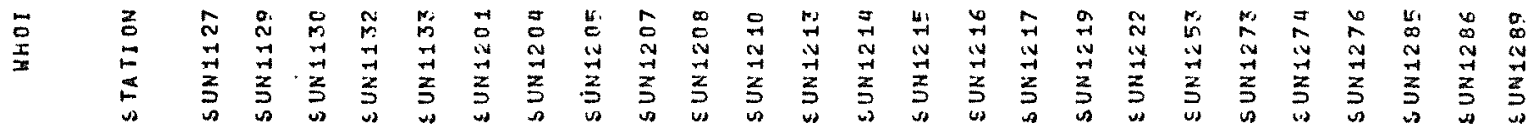




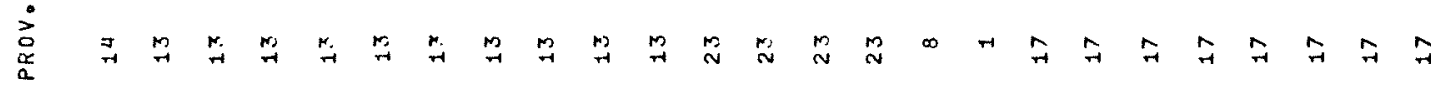

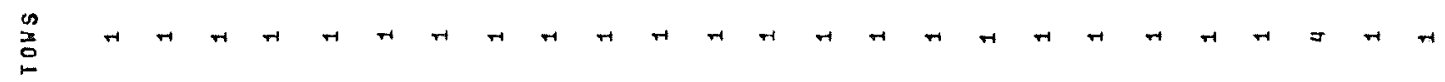

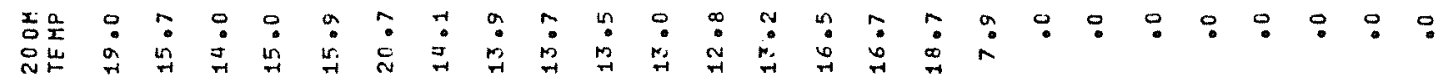

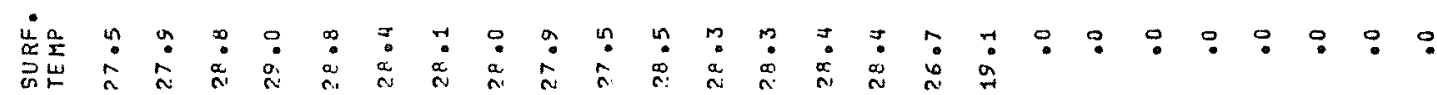

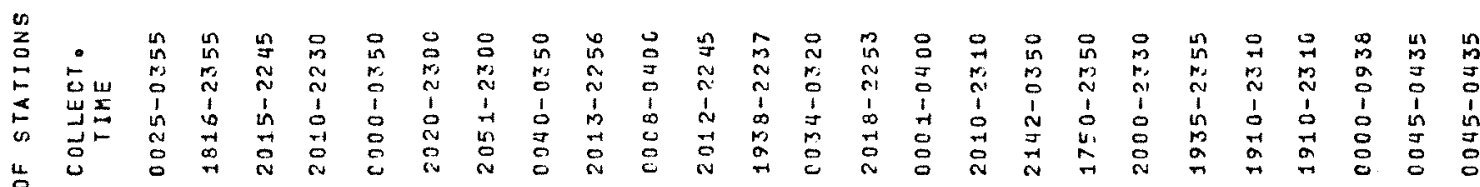

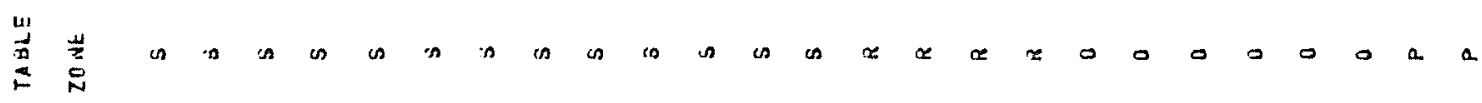

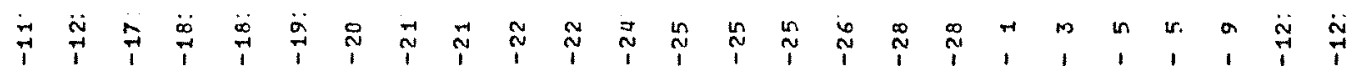

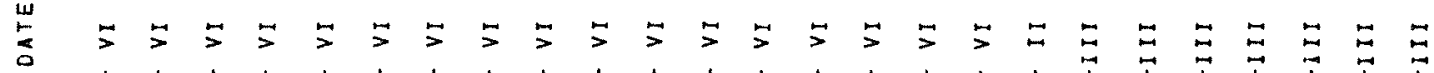

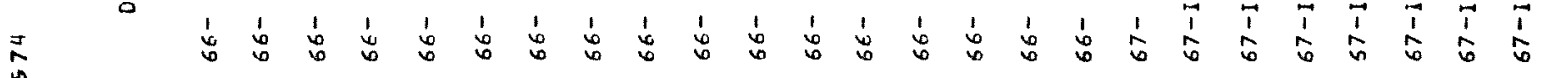

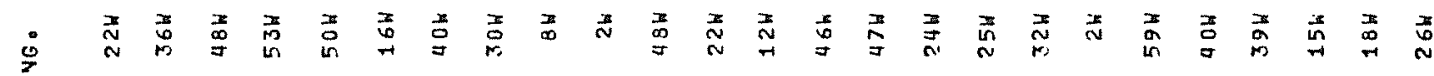

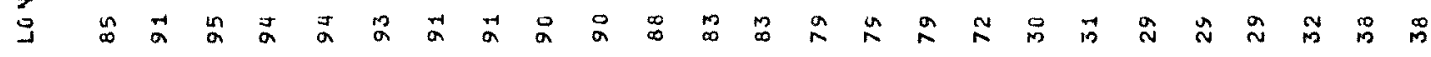
E

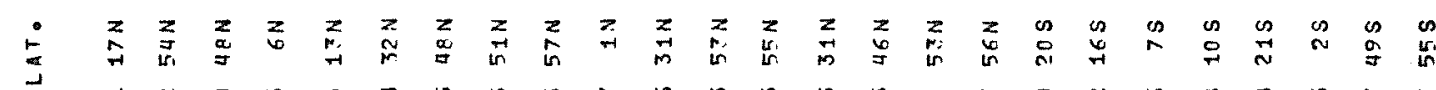

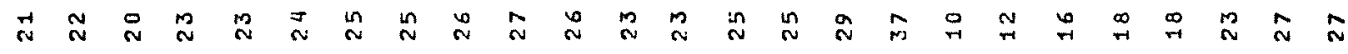

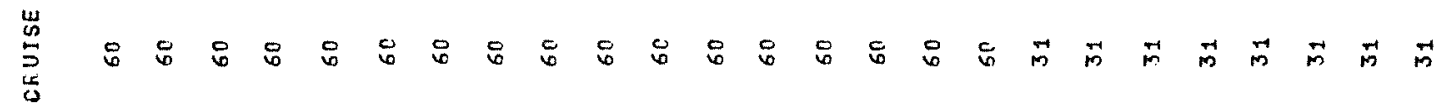

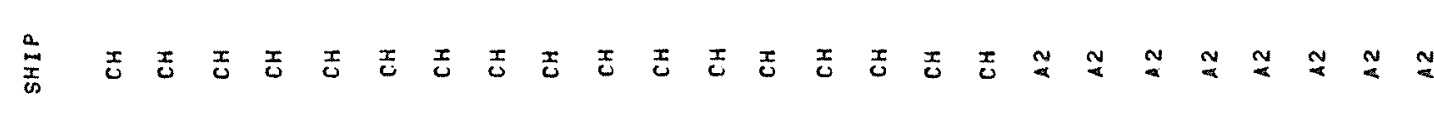

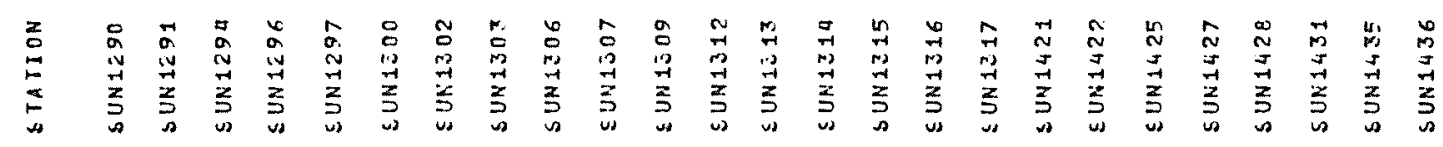




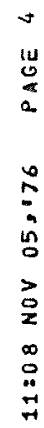

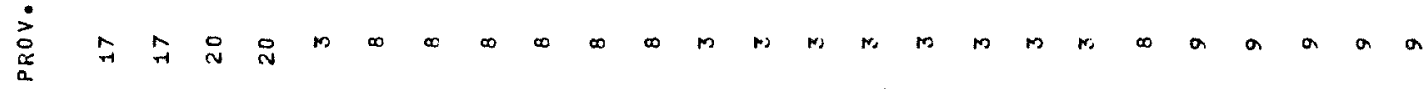

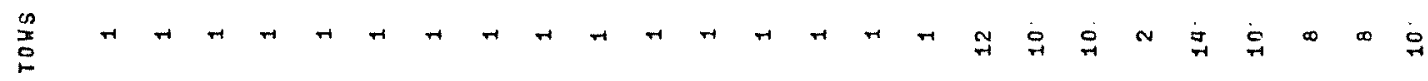

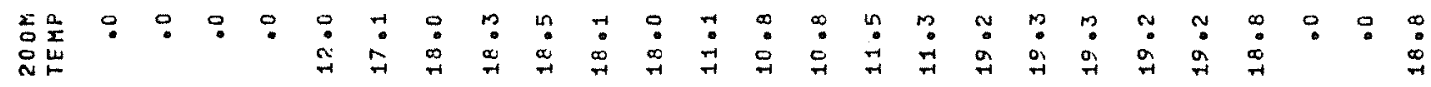

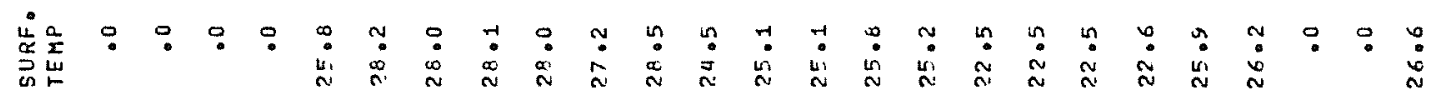

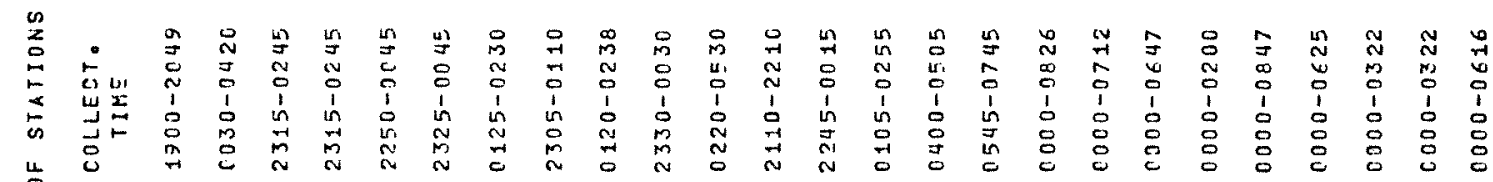
崌

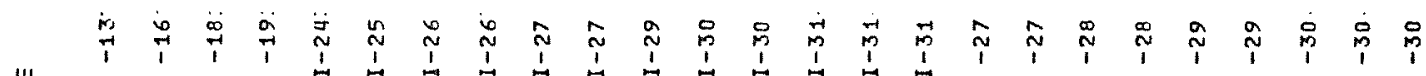

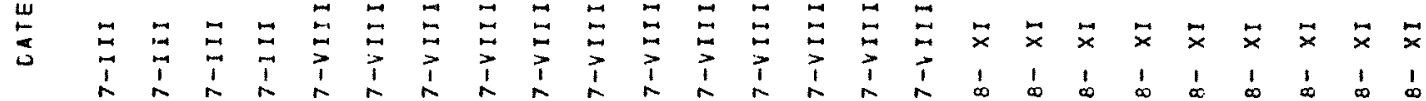

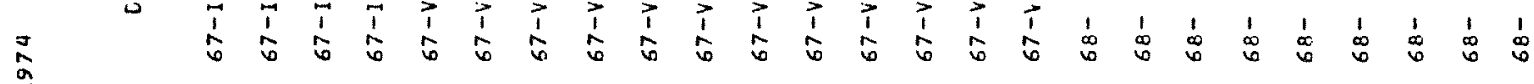

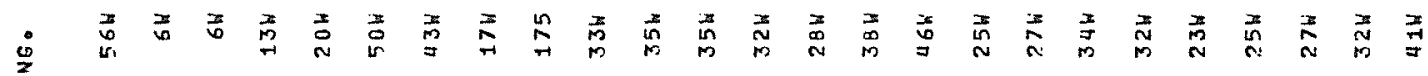

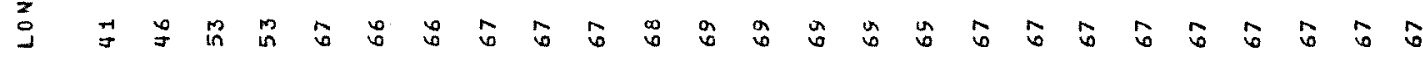
$\underset{5}{5}$

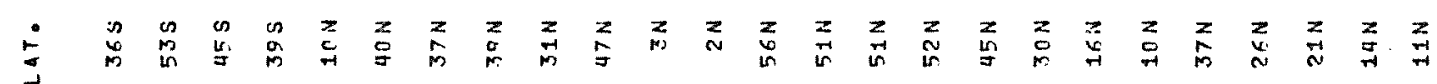

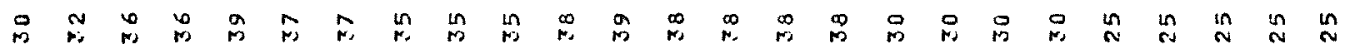

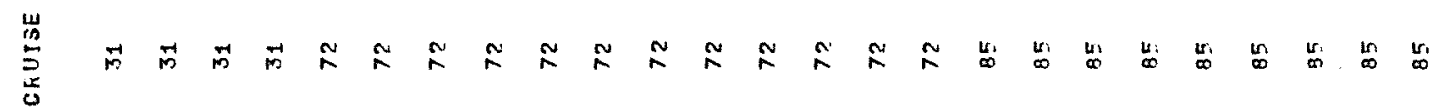
是

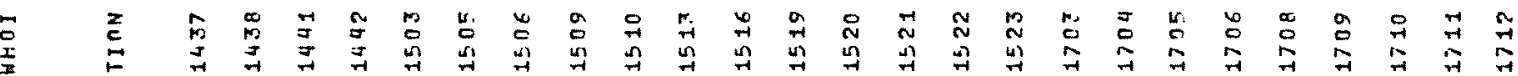

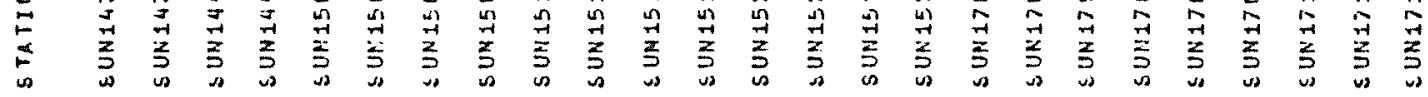


II-6

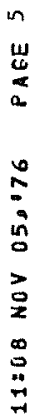

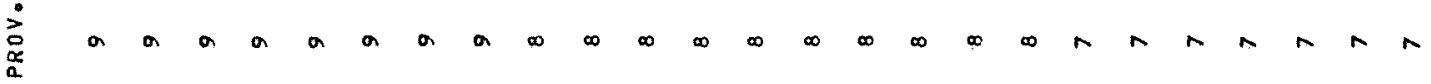
苔

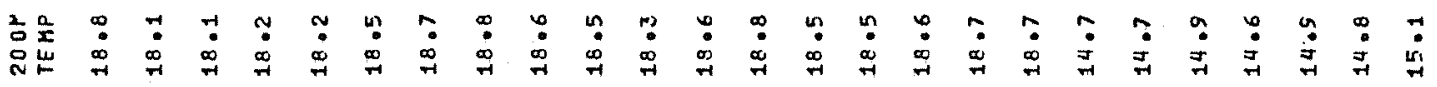

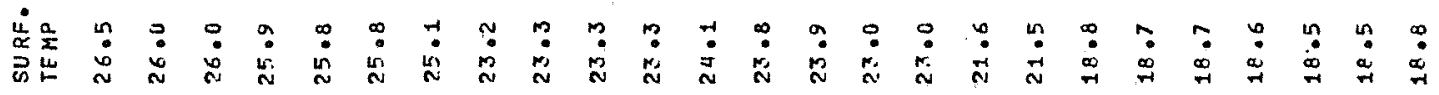

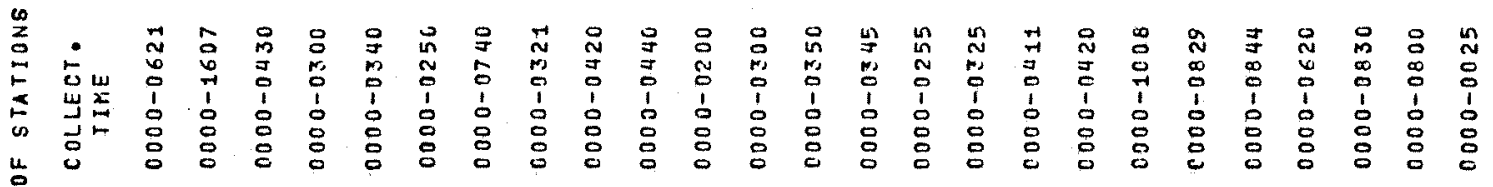

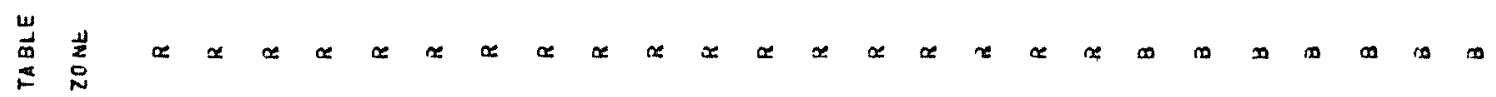
P.

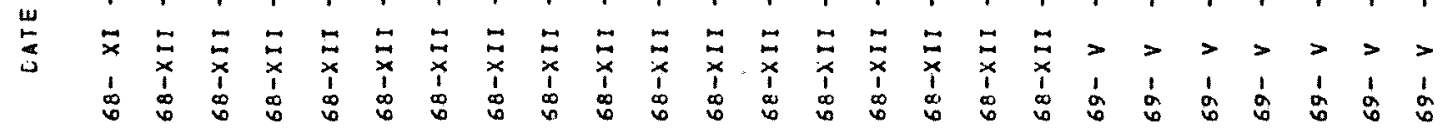

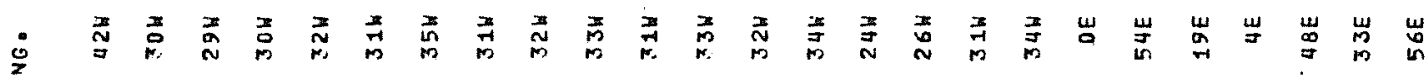

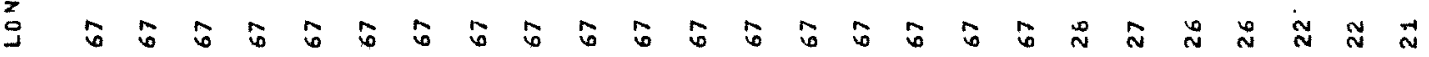
\begin{tabular}{l}
5 \\
0 \\
2 \\
0 \\
0 \\
\hline \\
0 \\
0
\end{tabular}

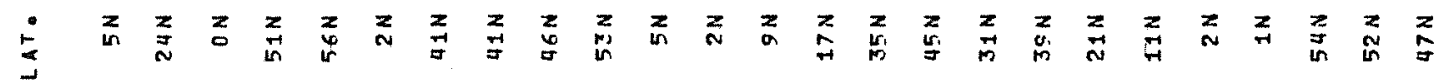

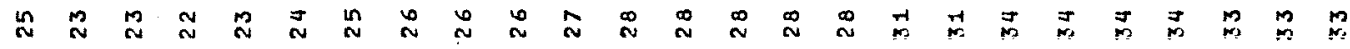

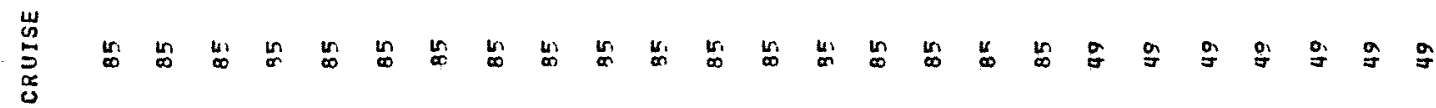
京焉

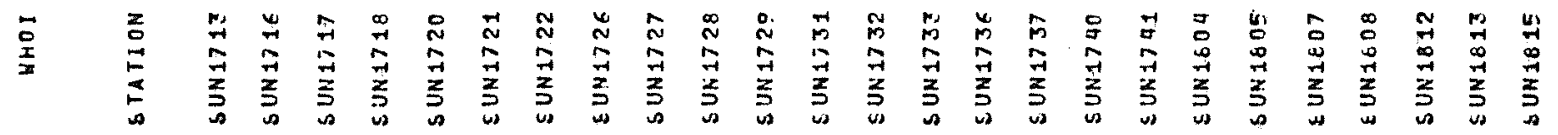


0
$u$
0
0
0

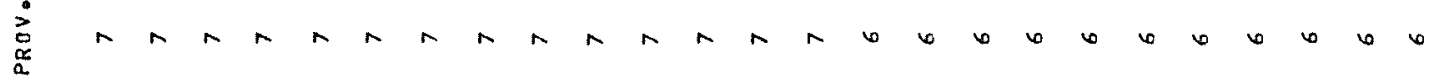

营

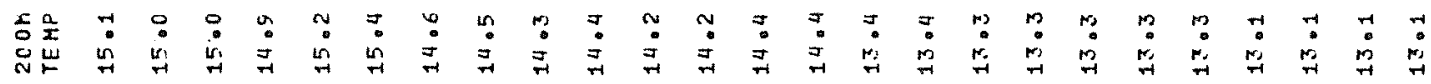

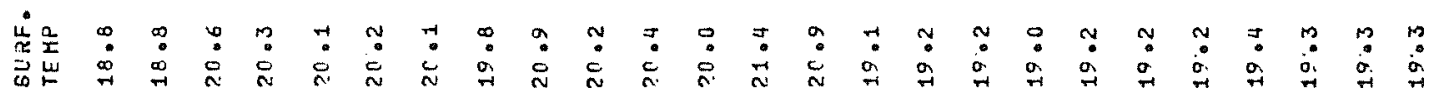

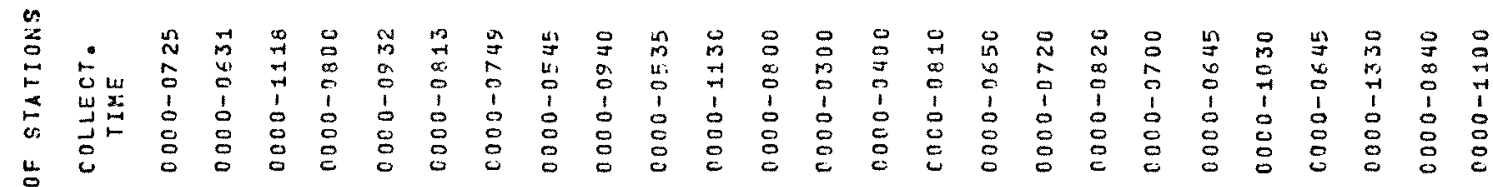

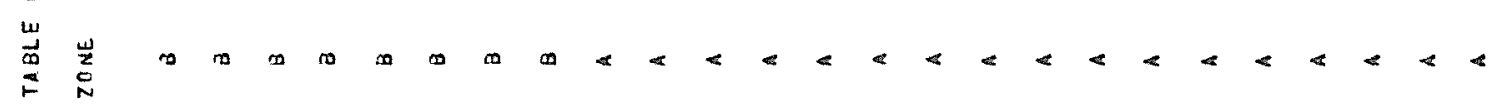

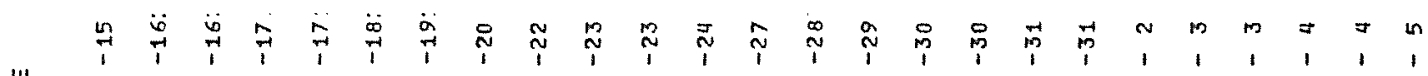

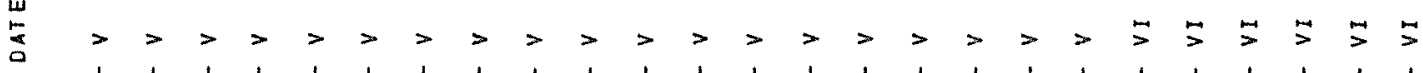

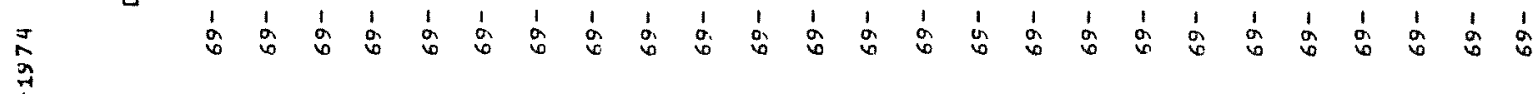

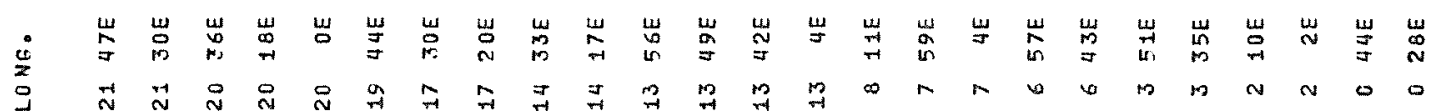

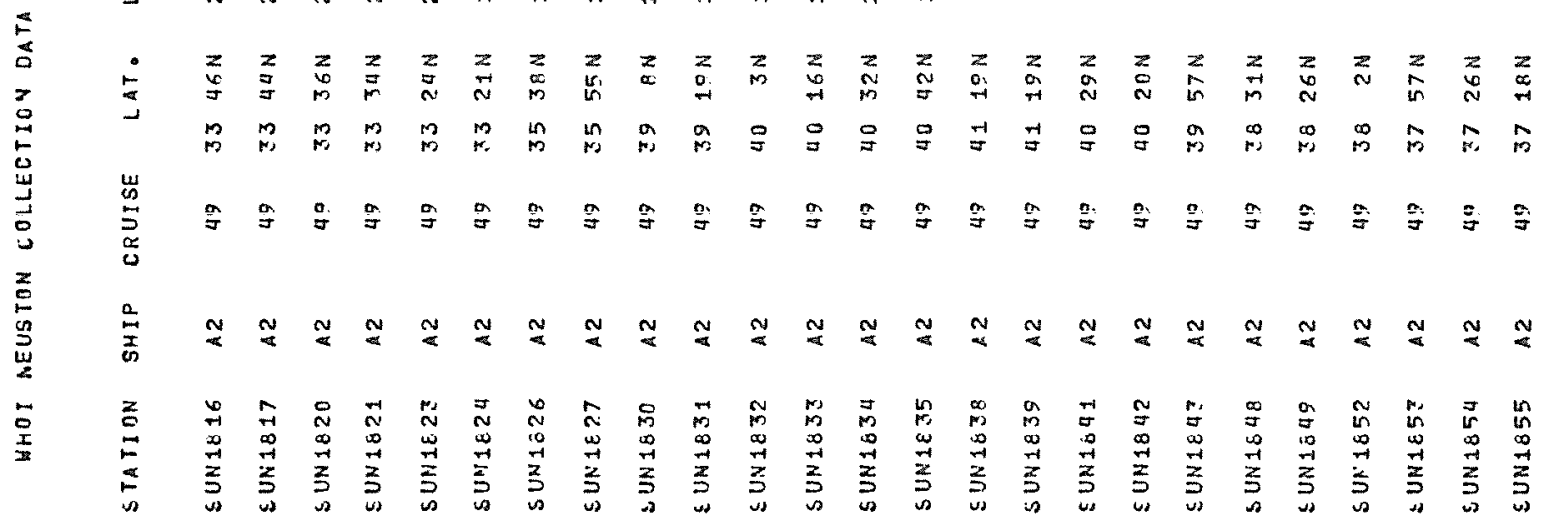




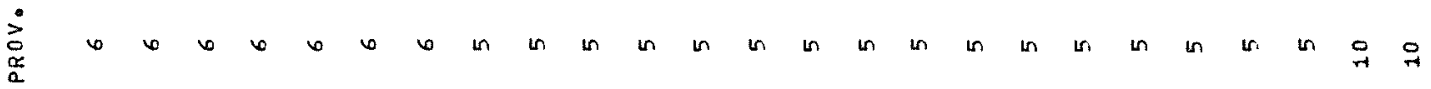

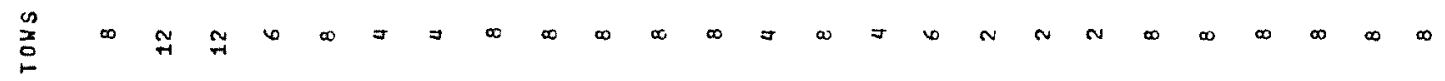

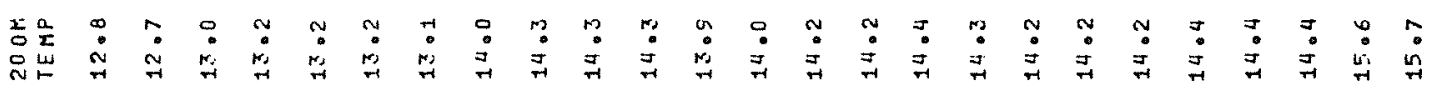

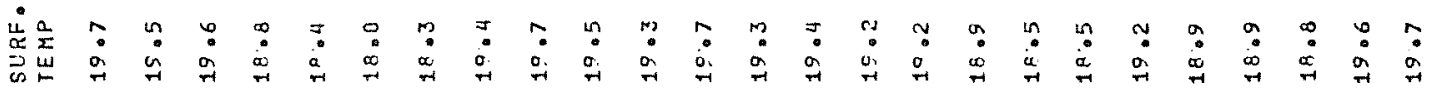

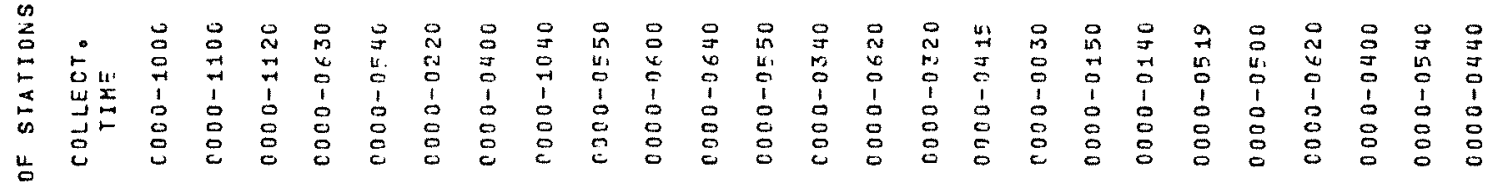

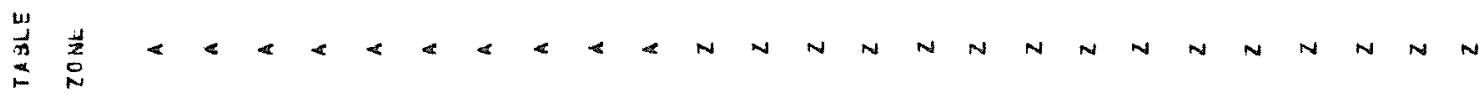

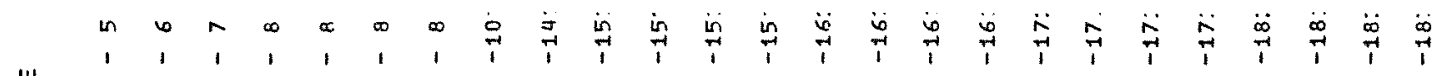

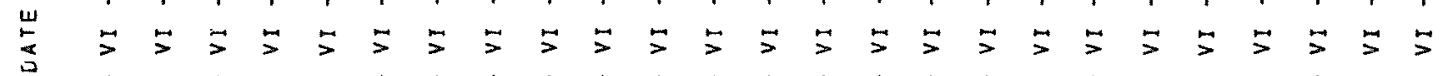

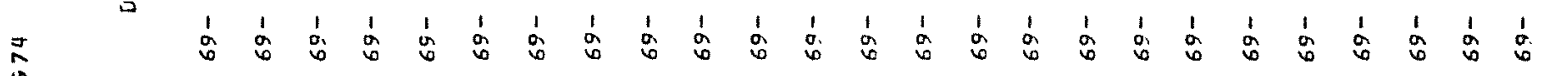

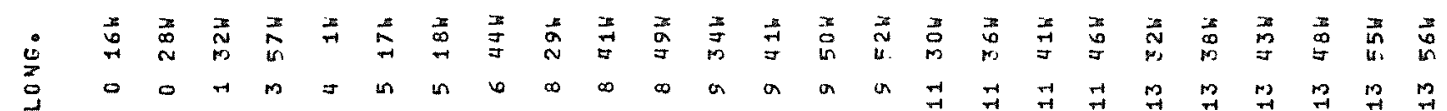

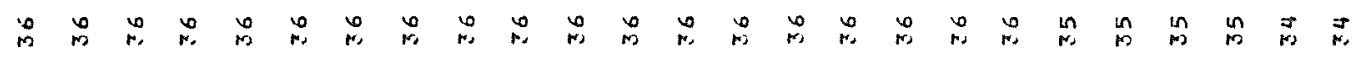

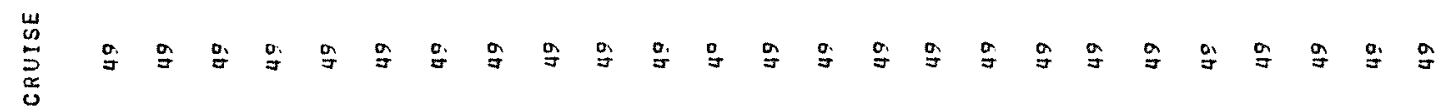

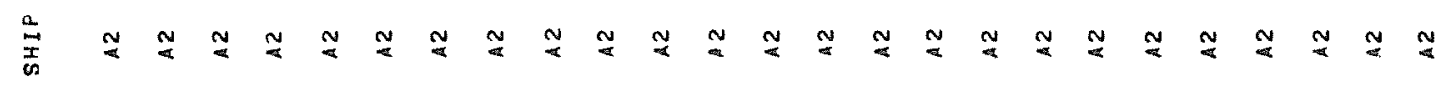

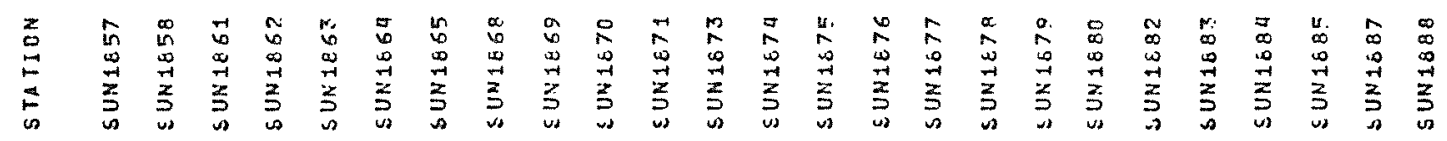




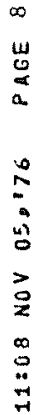

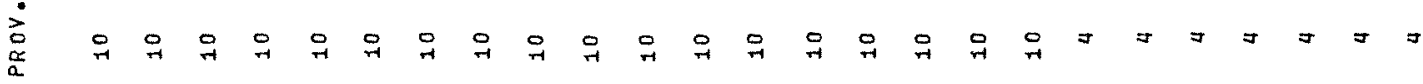

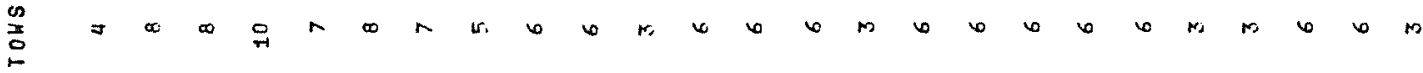

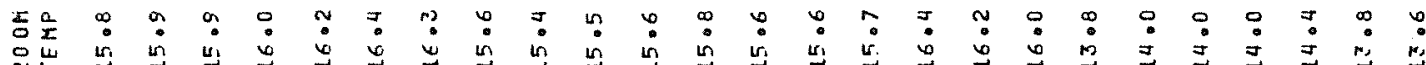

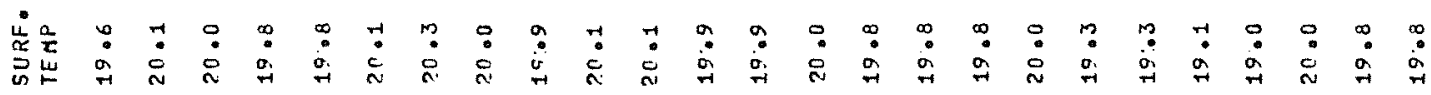

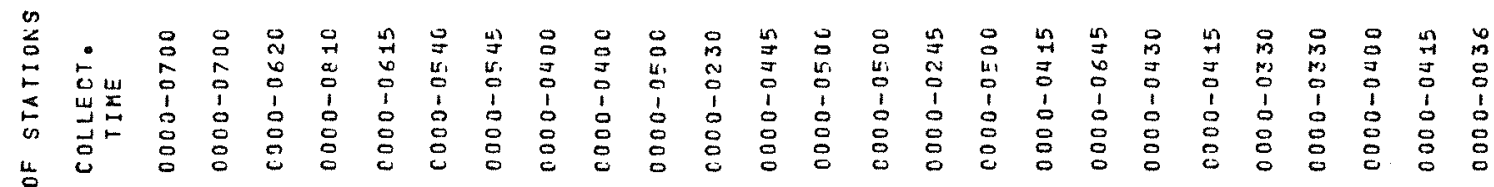
邖

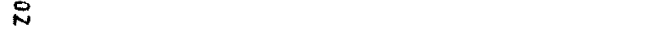

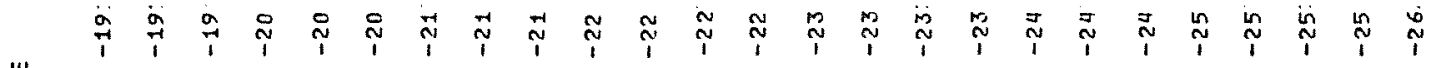

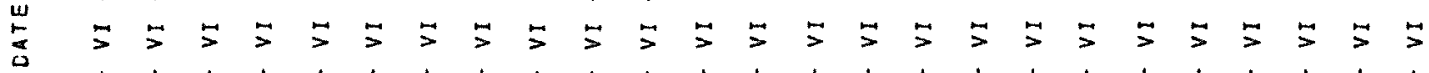

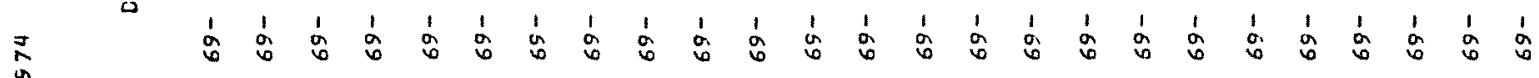

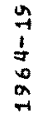

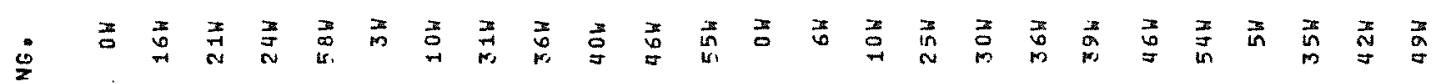

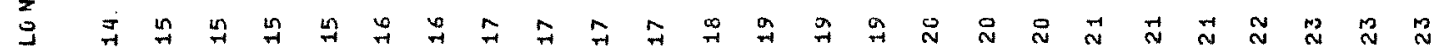
5

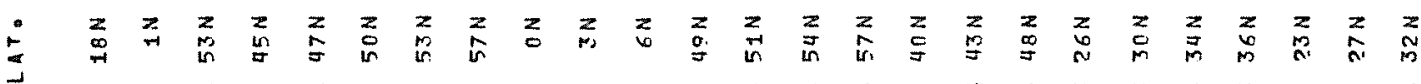

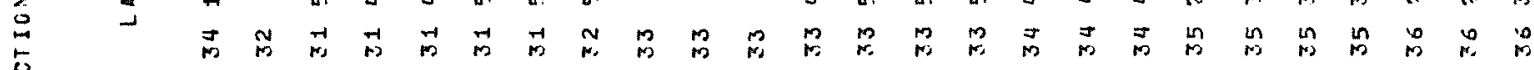

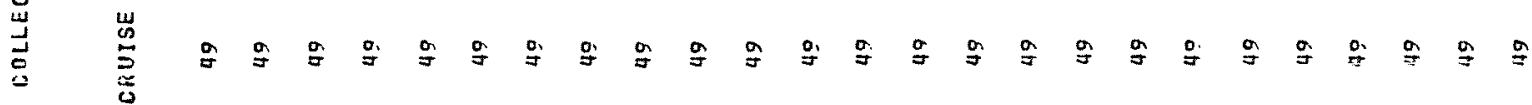

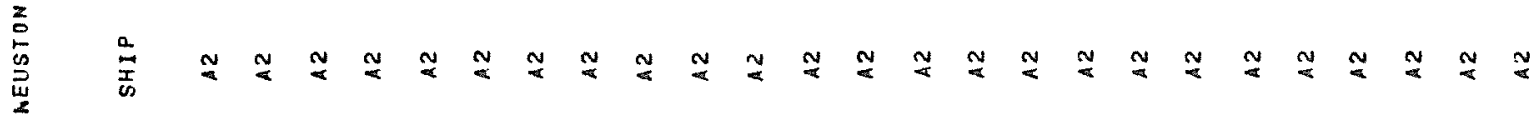

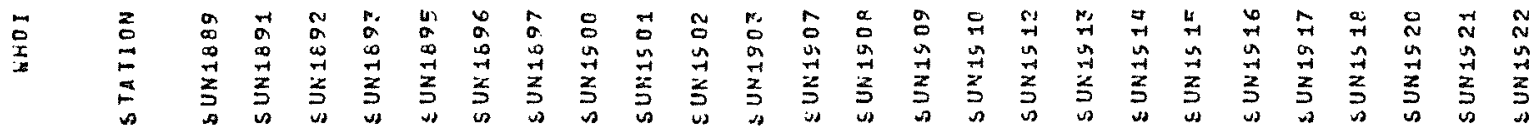




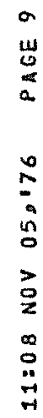

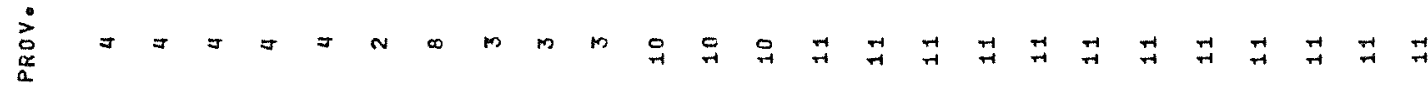

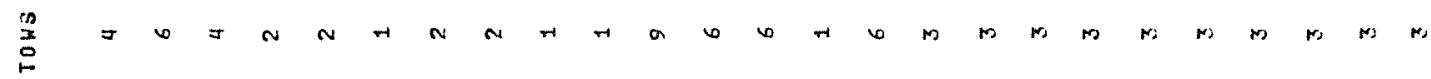

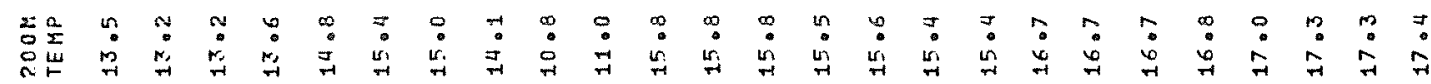

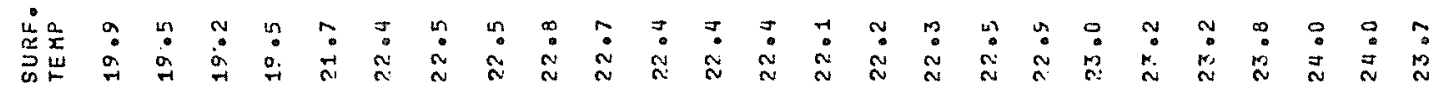

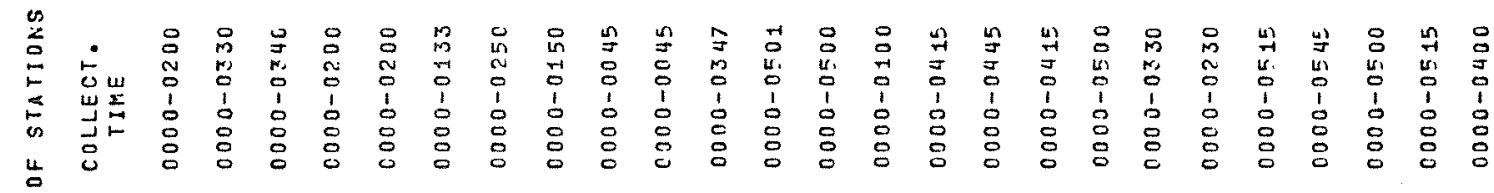

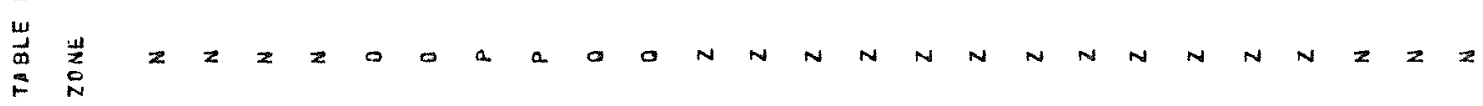

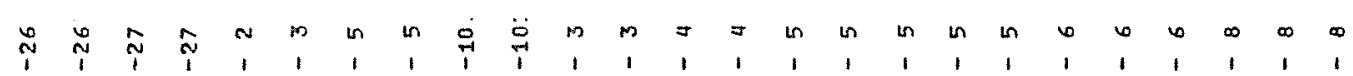

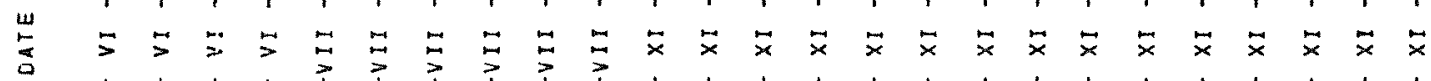

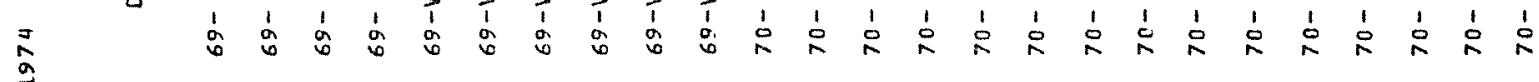

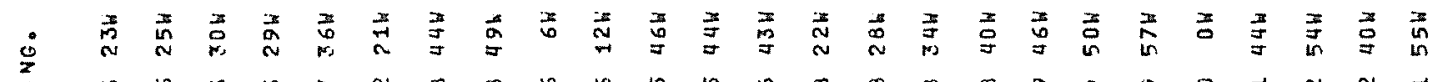
$\underline{5}$

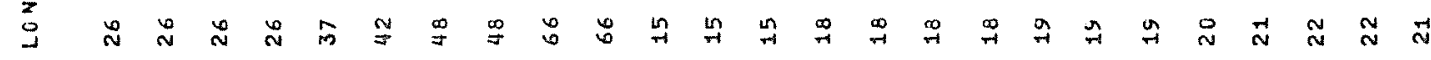

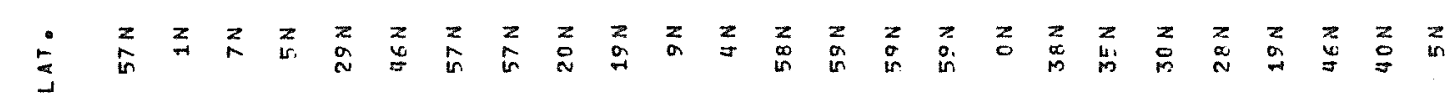

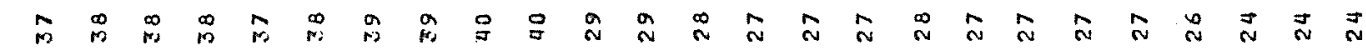

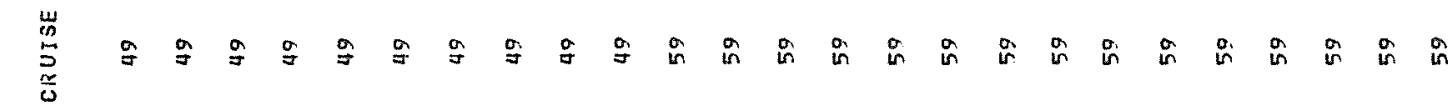
焉

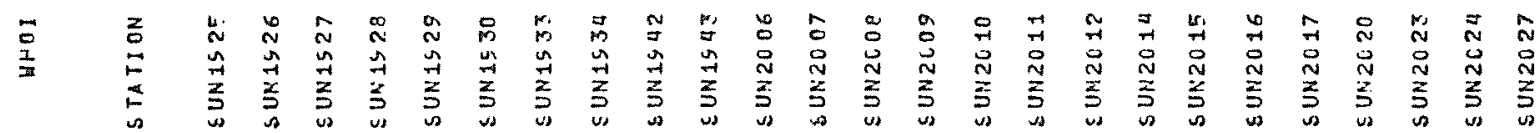




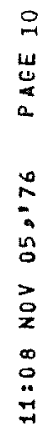

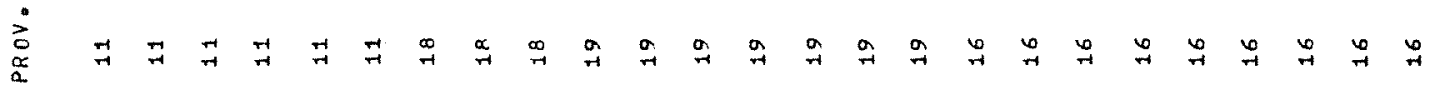
5
0
0

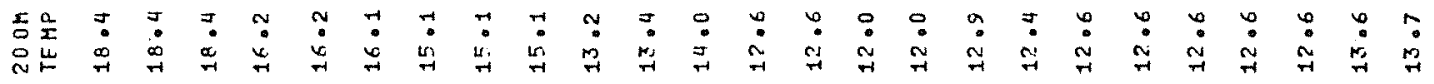

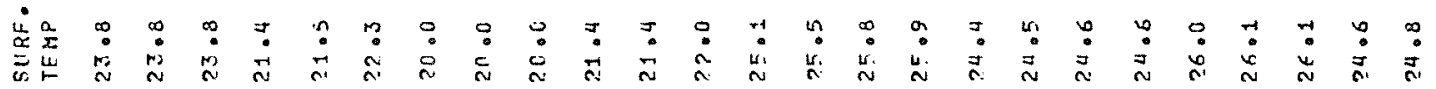

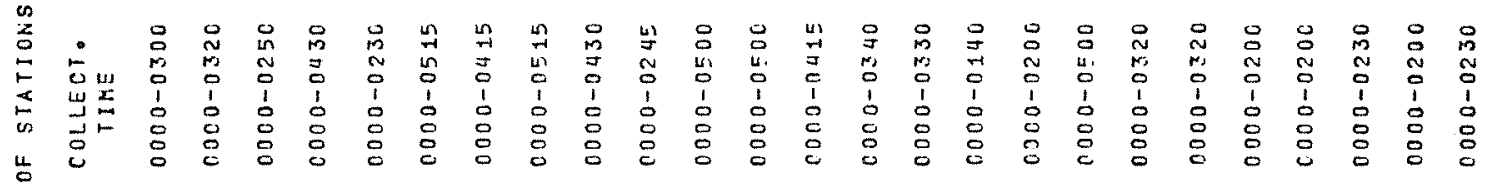
岩岩

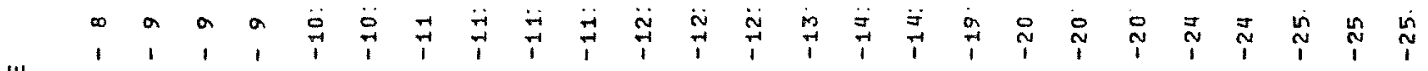

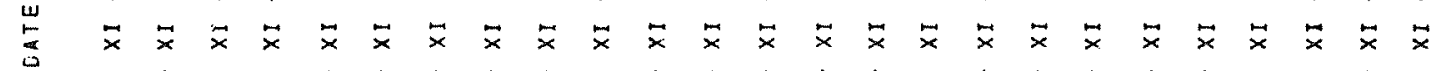

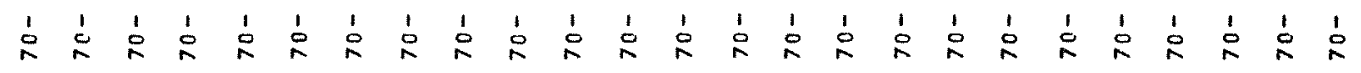

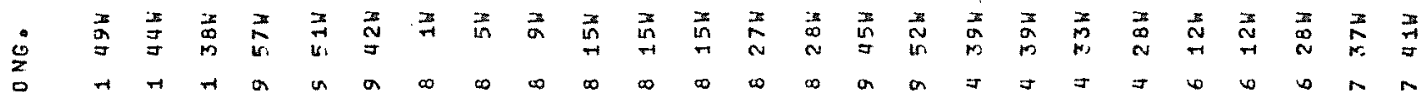

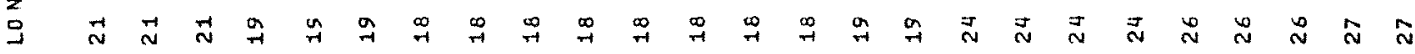
E

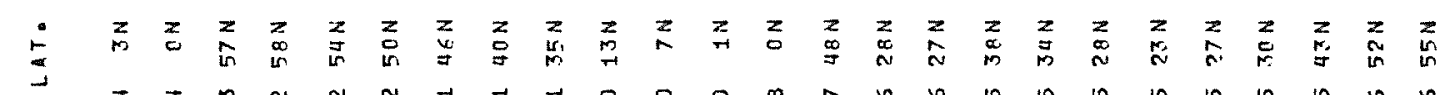

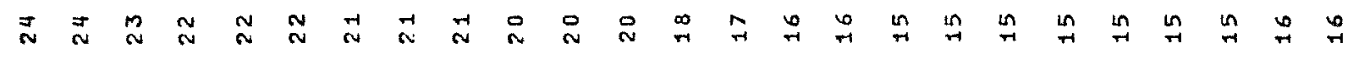

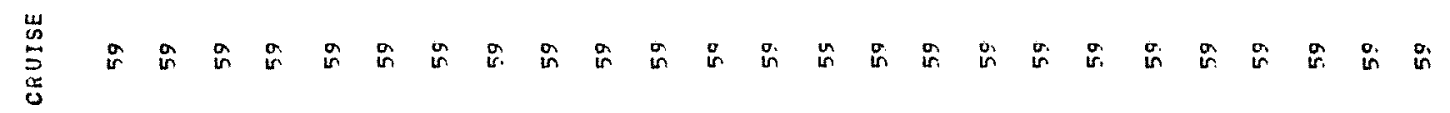

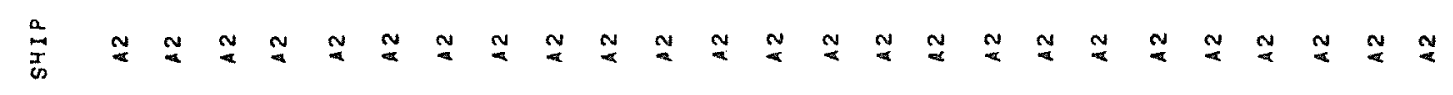

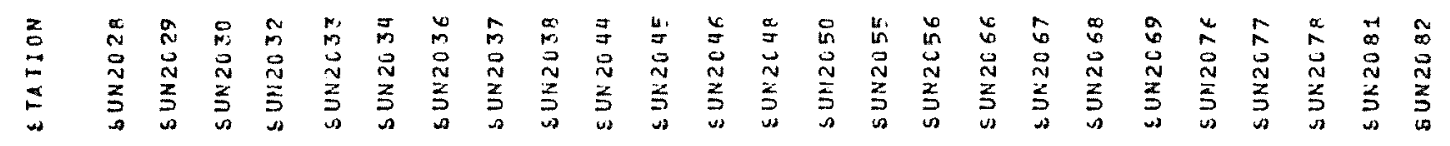


II -12

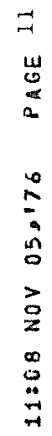

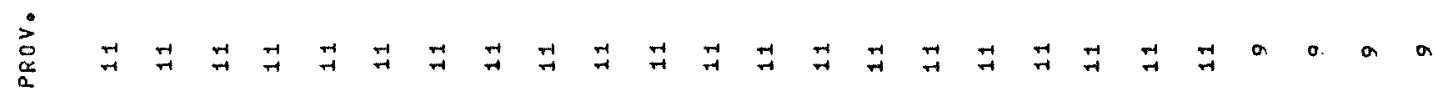

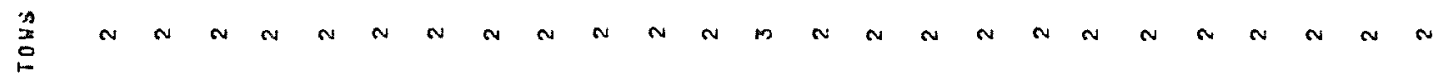

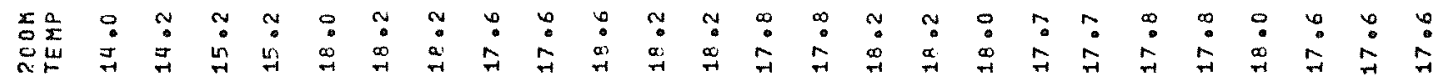

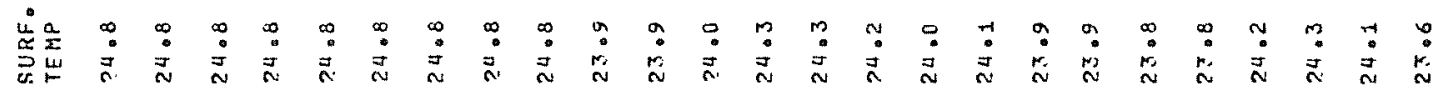

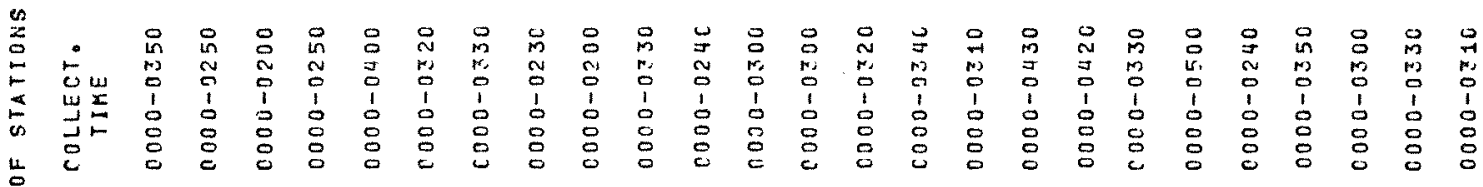

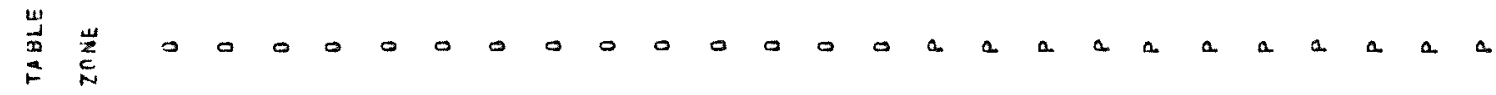

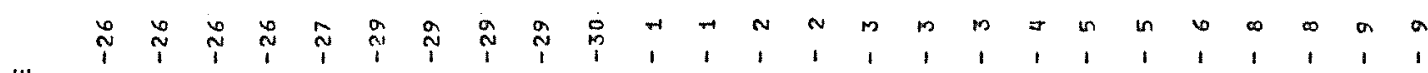

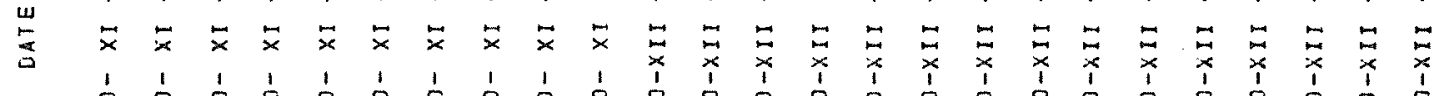

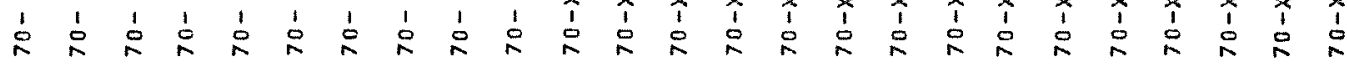

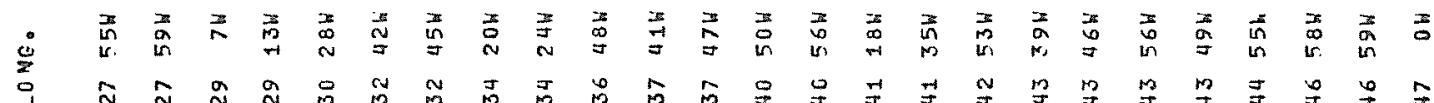

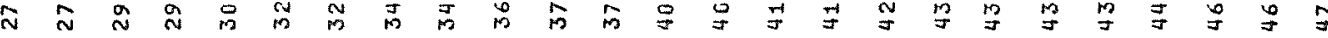

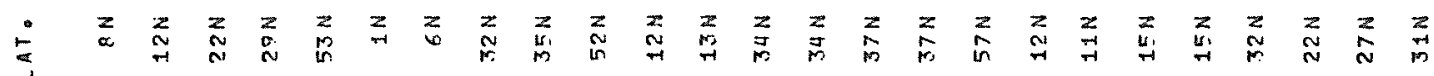

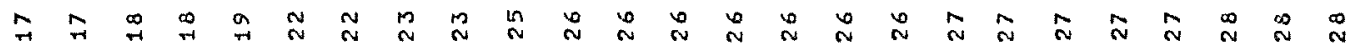

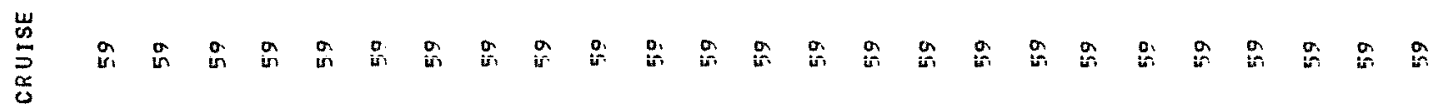

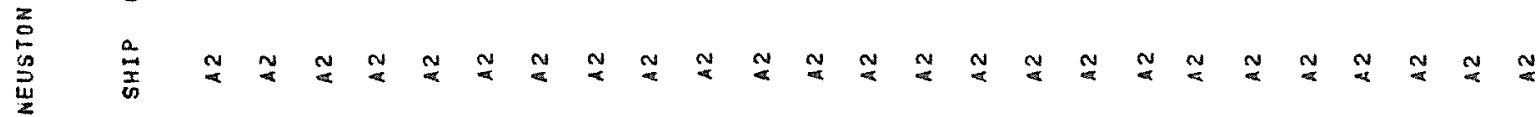

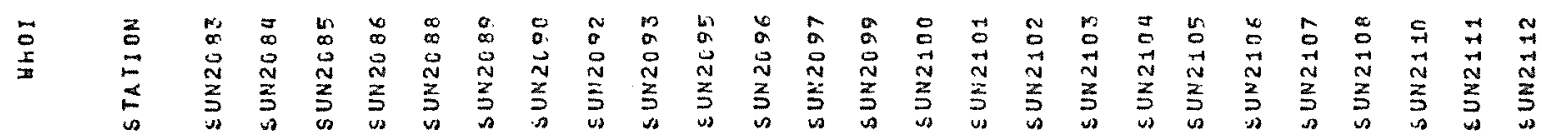


$\mathrm{II}-13$

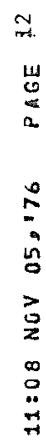

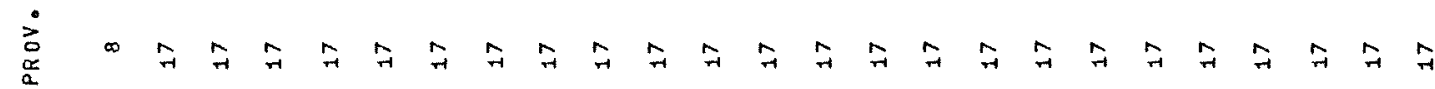

0
0

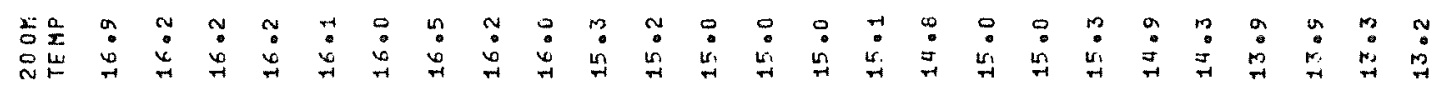

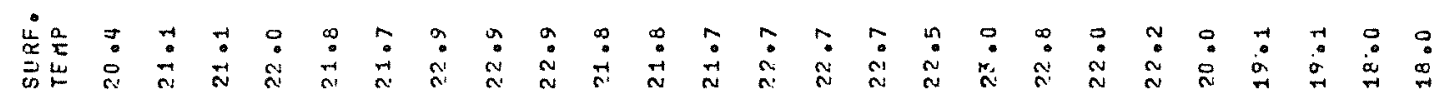

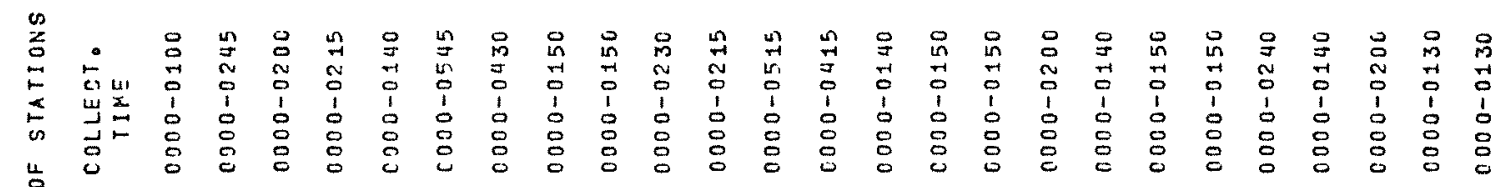

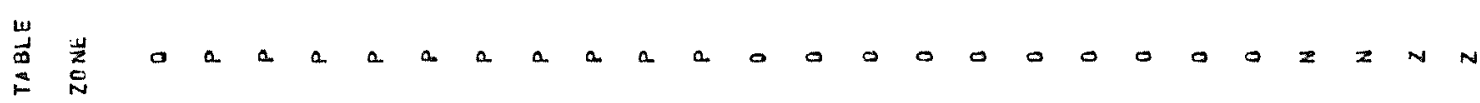

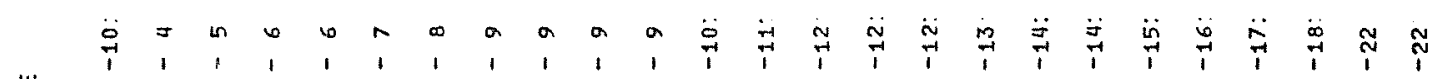

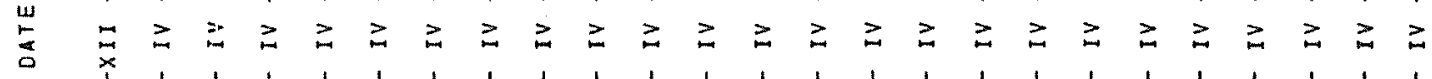

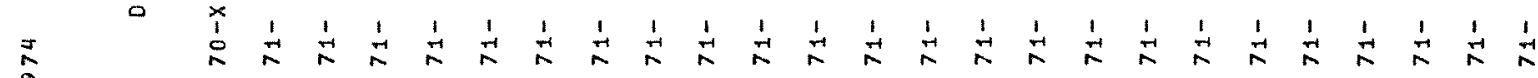

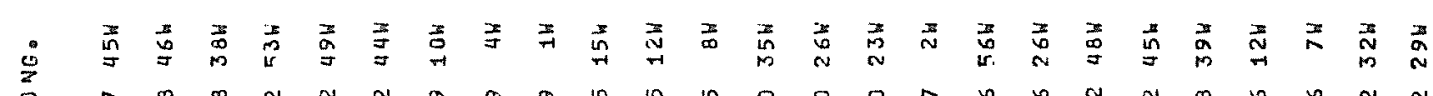

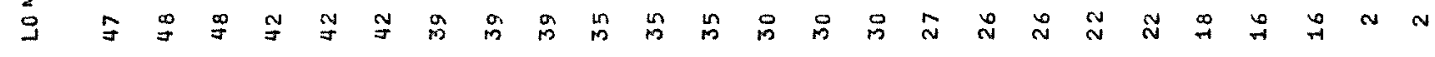

5
0
$z$
0
0
0
$u$
0
0

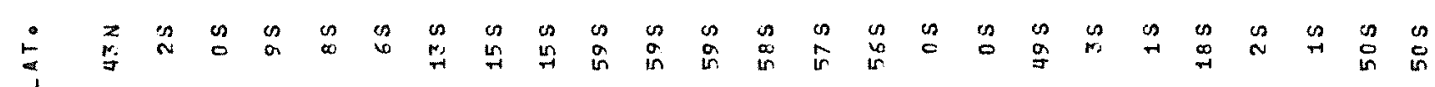

m

岕

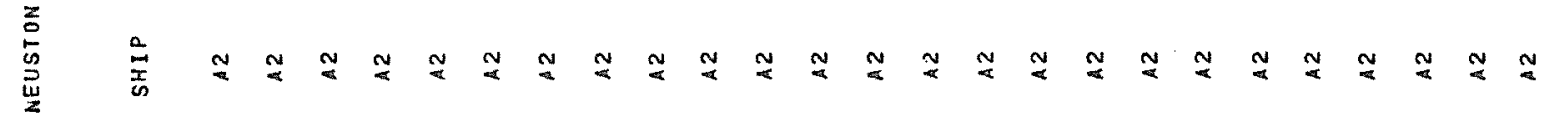

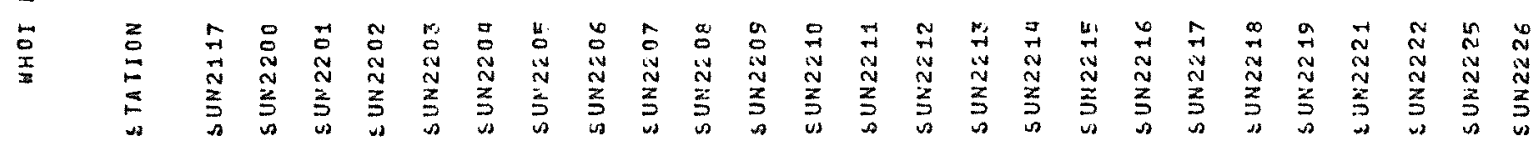




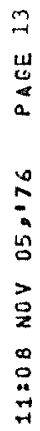

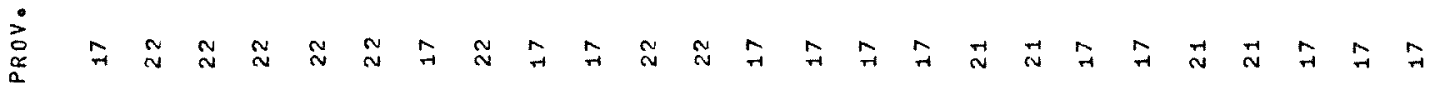

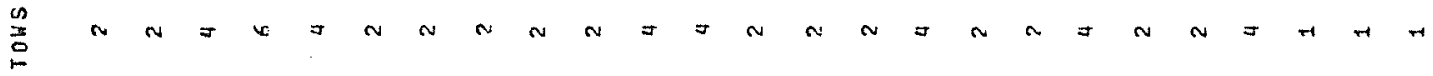

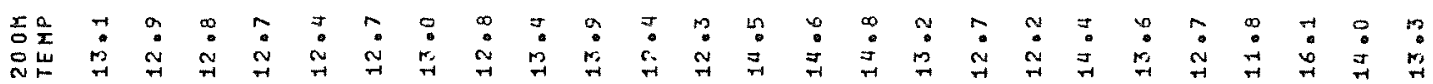

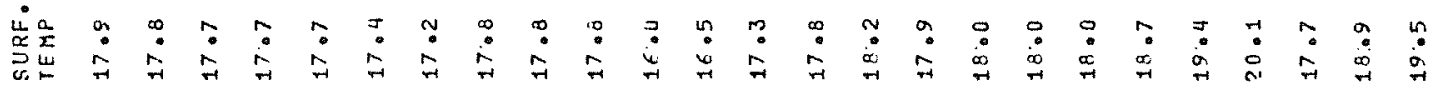

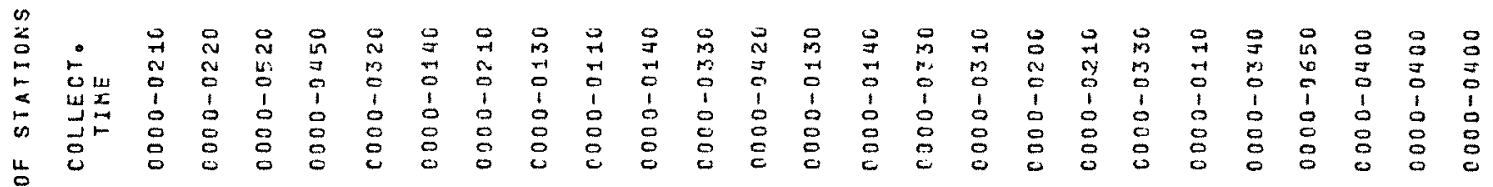
崖苦N

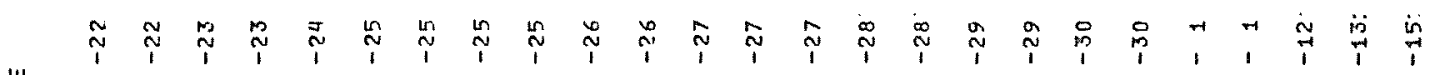

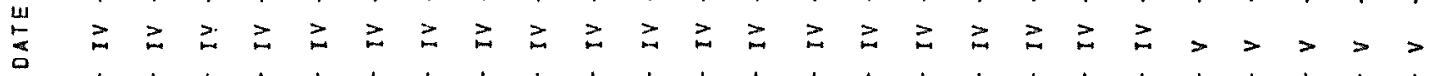

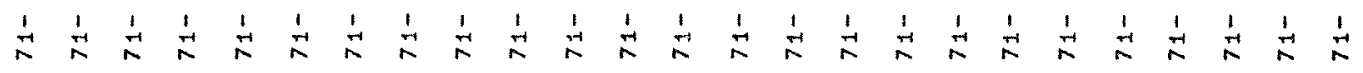

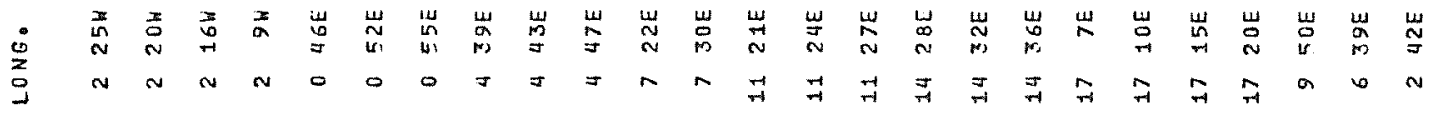

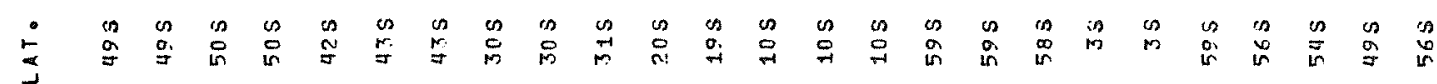

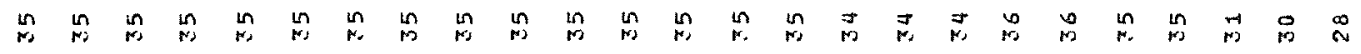

岁 萦

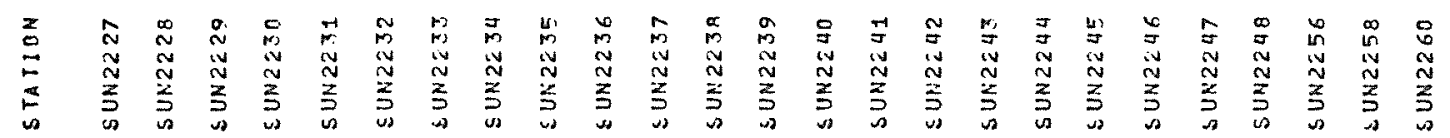


5
$\vdots$
0
0
0
0
$\vdots$
$\vdots$
0
0
2
2
0
0
0

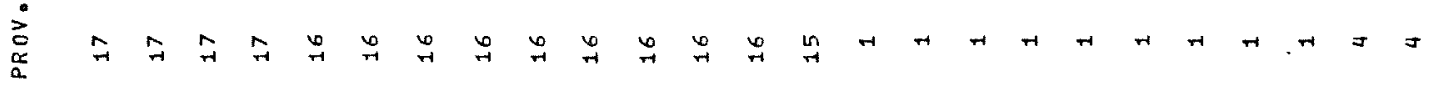
亮

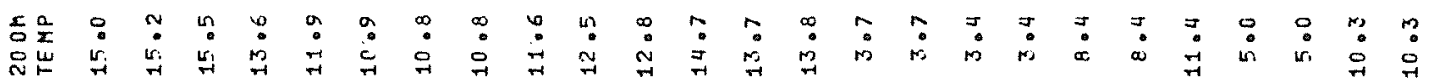
崖密

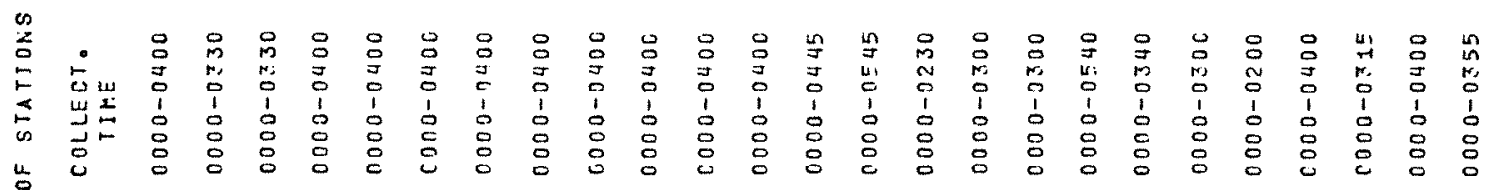

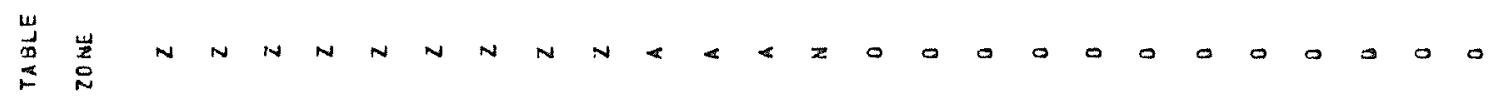

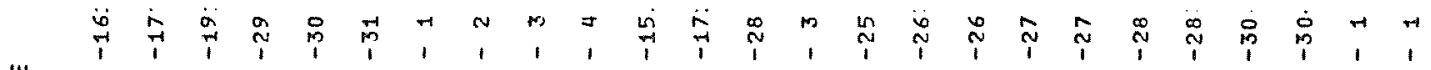

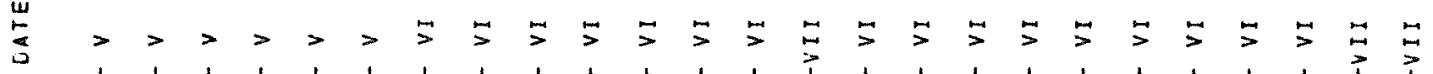

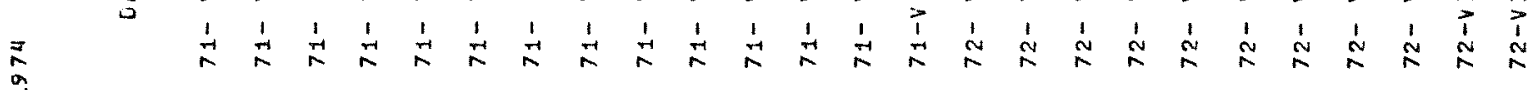

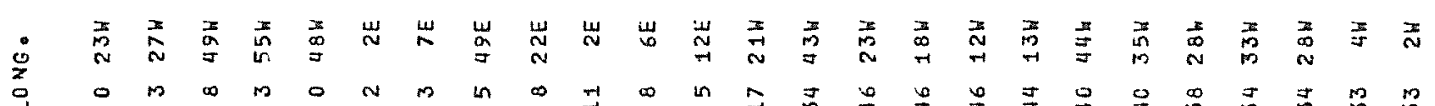

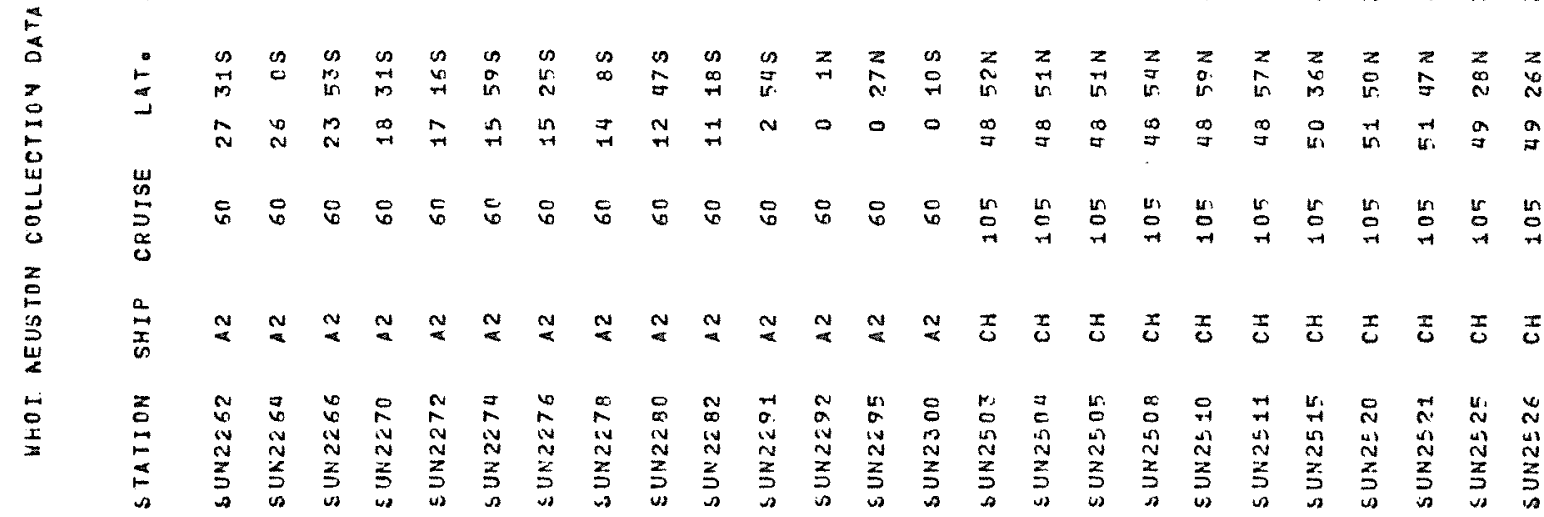


$n$
-4
0
0
0
0
$: 0$
$\vdots$
$\vdots$
0
0
0
0
0
0
0
0
0

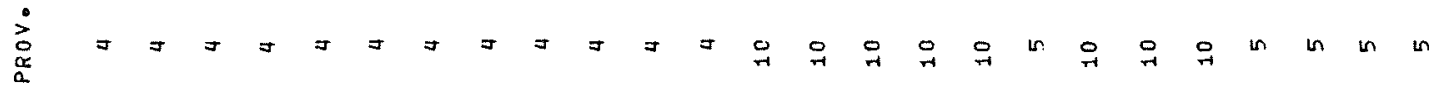
总

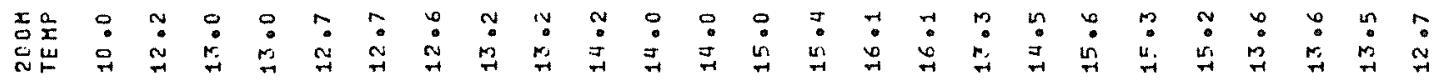

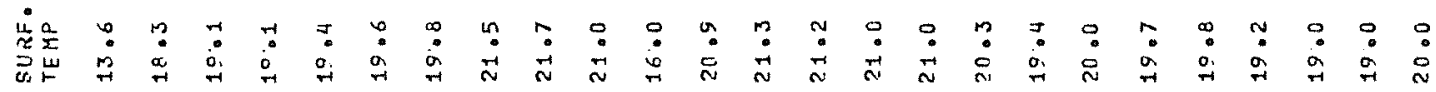

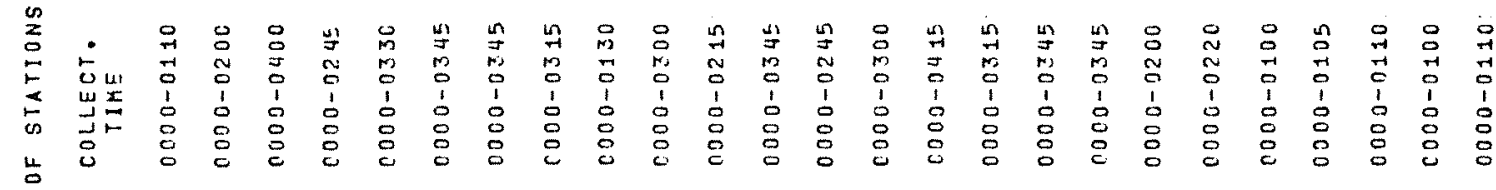

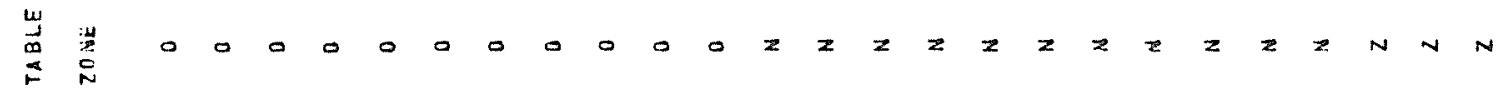

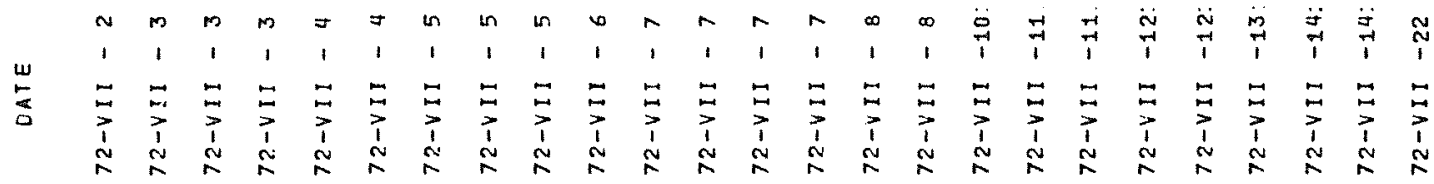

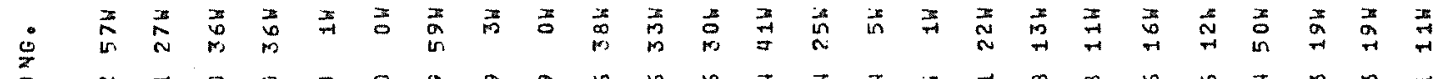

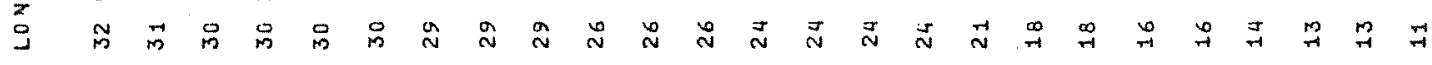
$\stackrel{5}{0}$

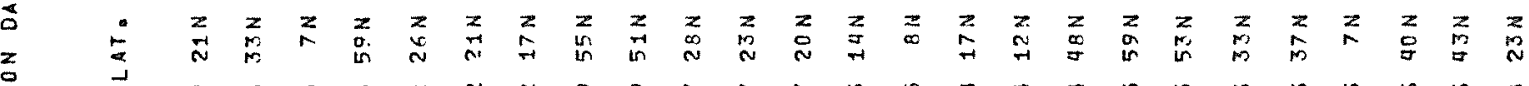

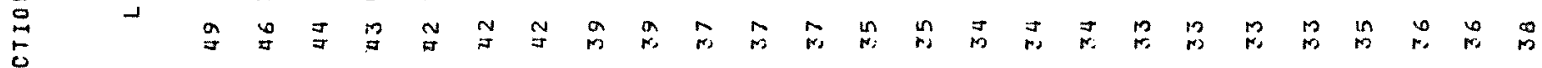

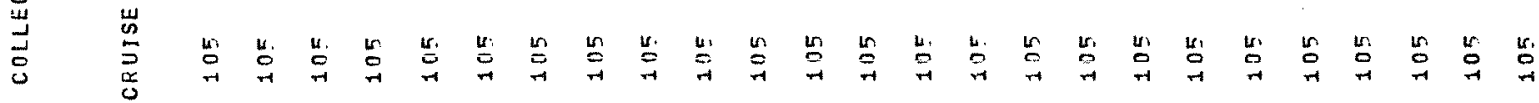
羔

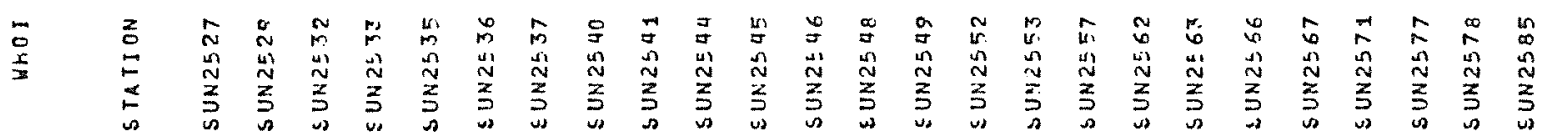




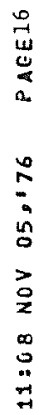

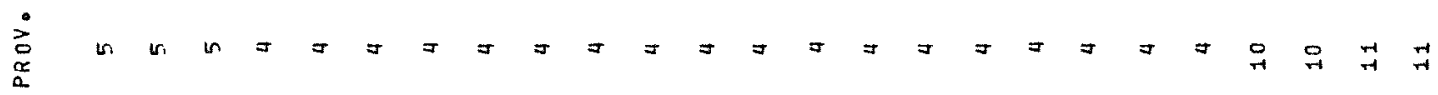

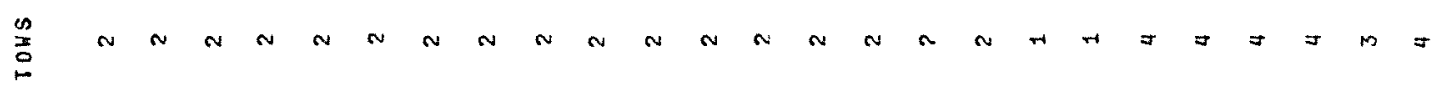

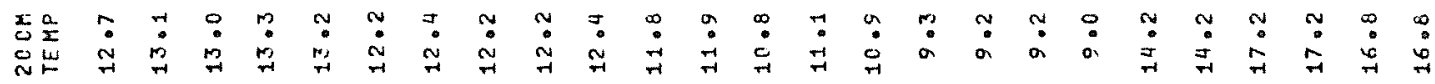

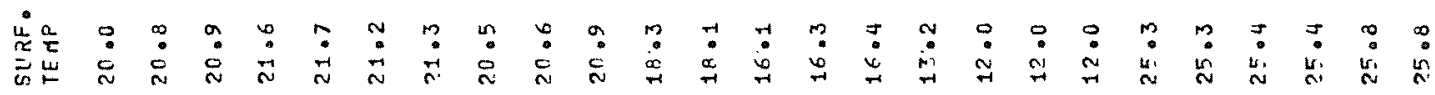

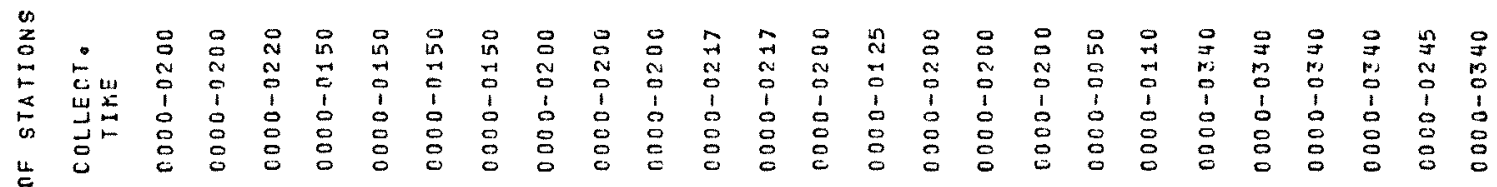

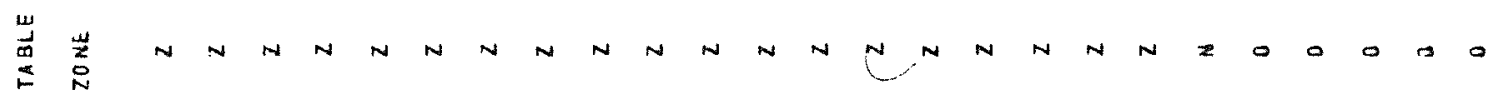

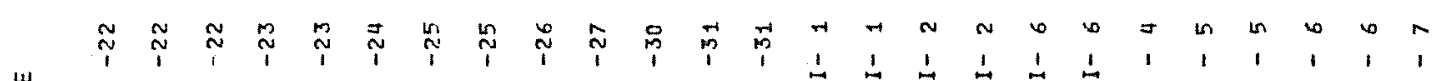

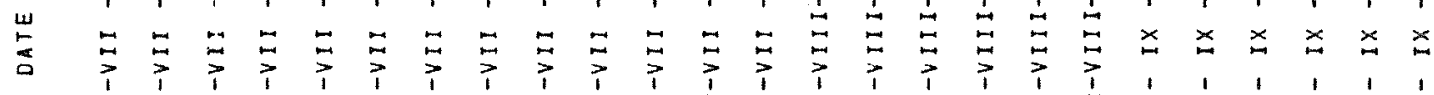

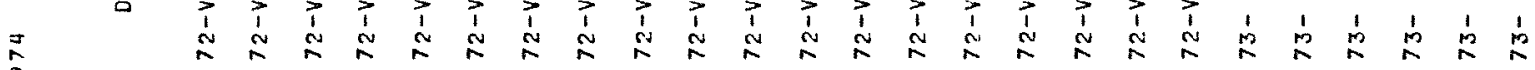

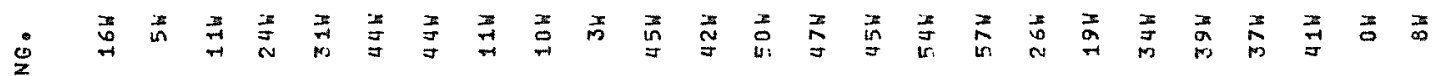

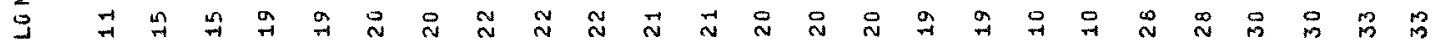

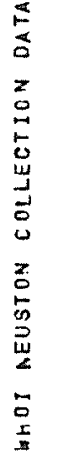

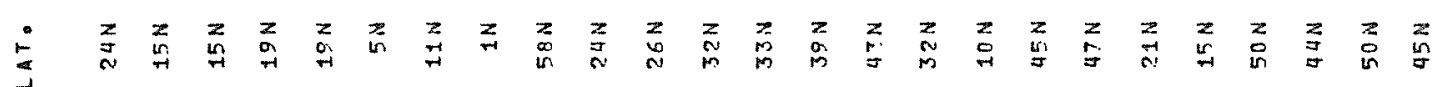

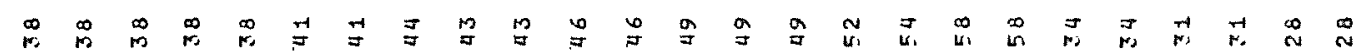

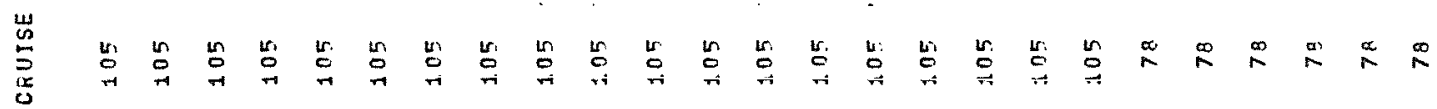
焉 I I I I I

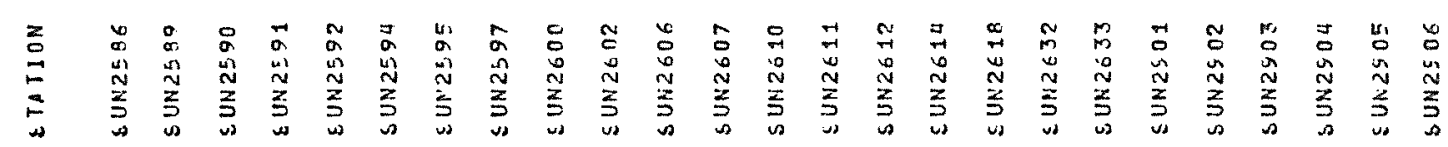


2
ü
$\vdots$
0

递 总 J

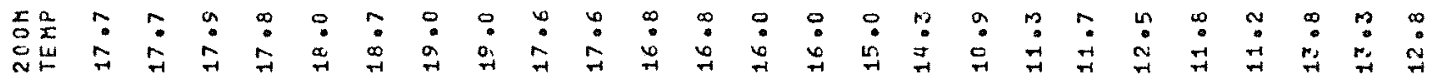

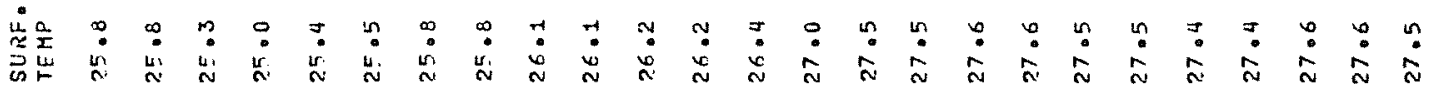

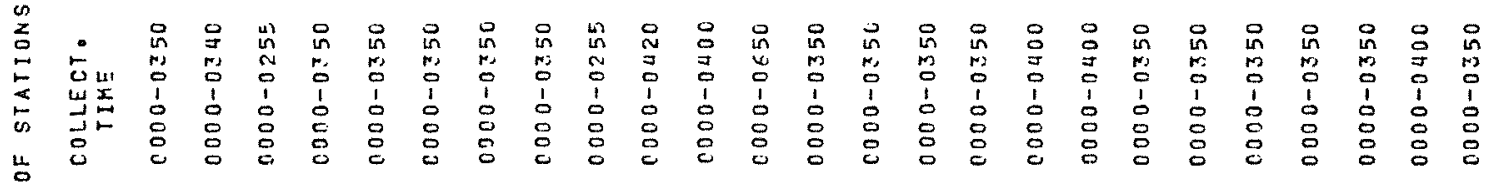

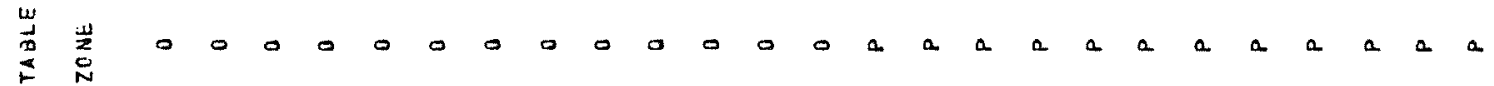

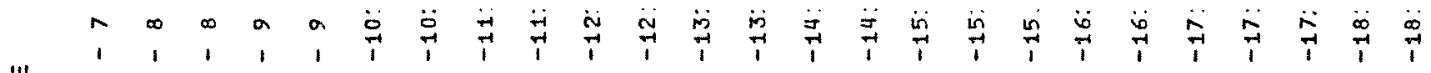

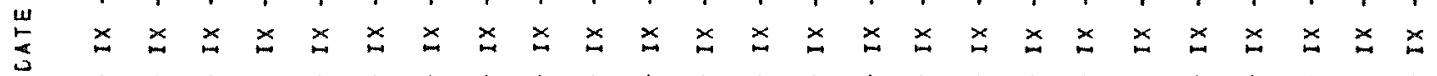

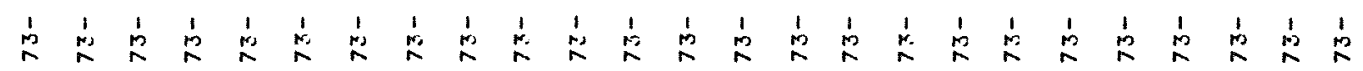

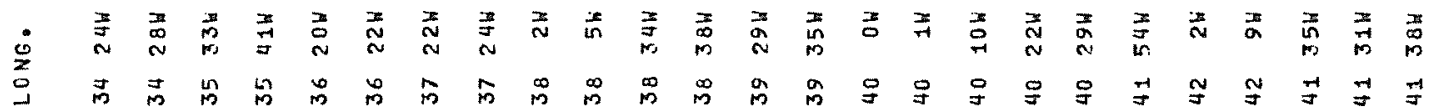

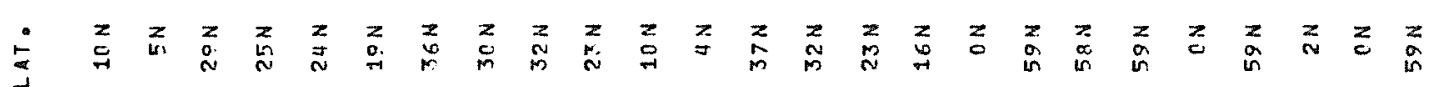

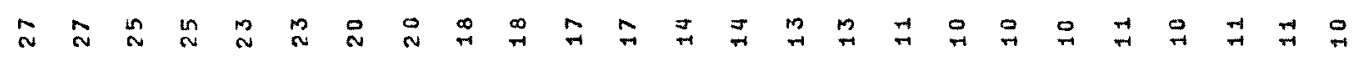

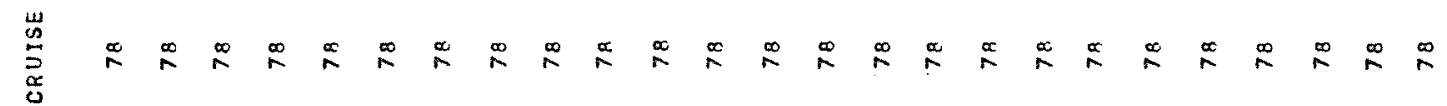

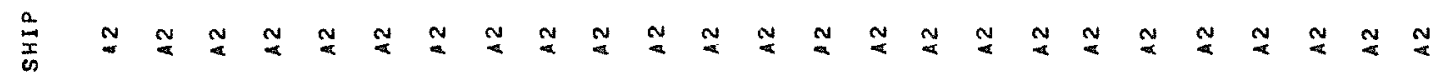

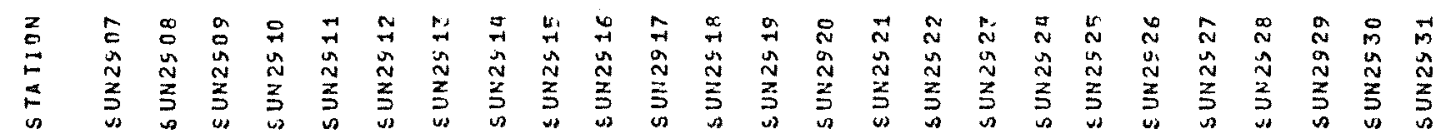




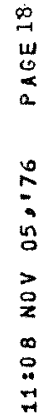

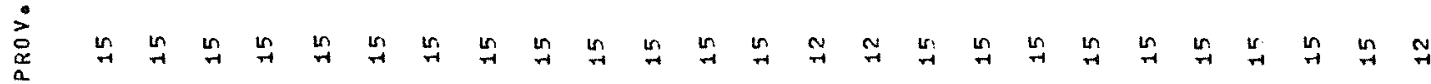

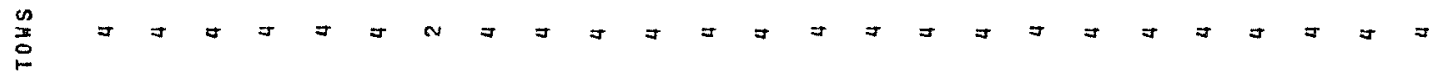

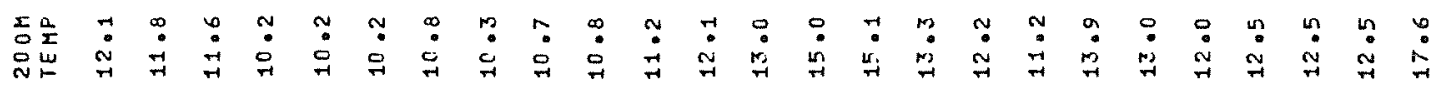

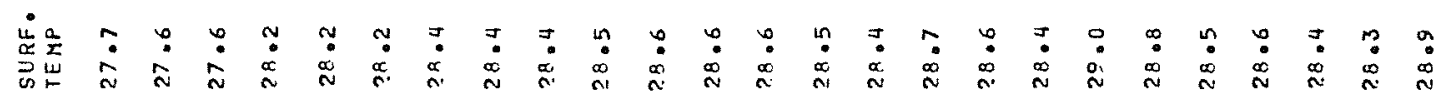

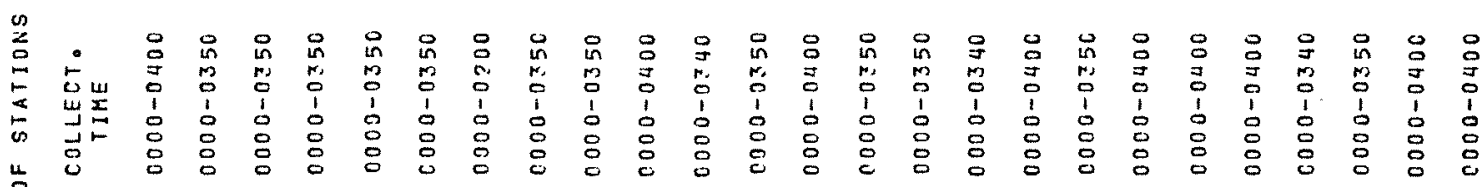

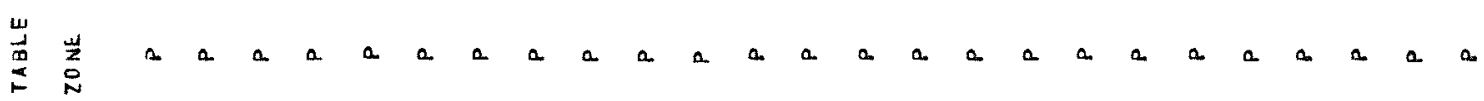

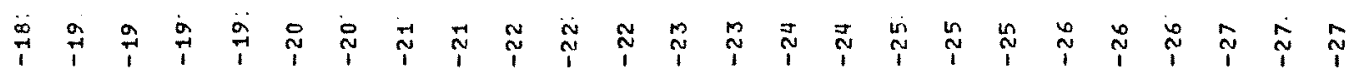

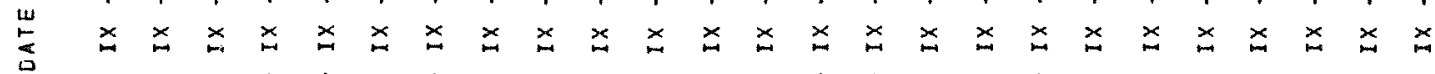

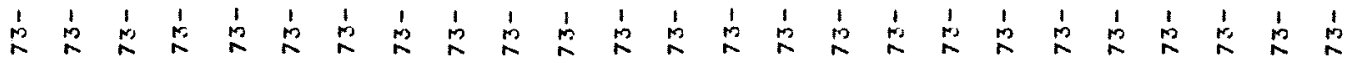

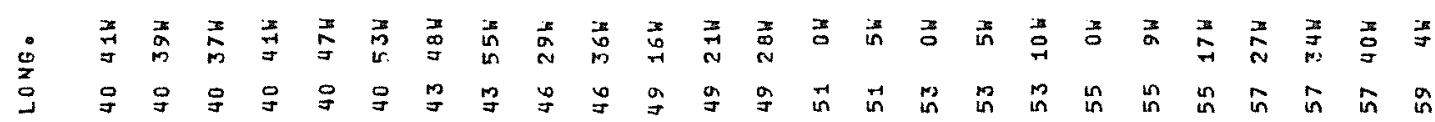
s

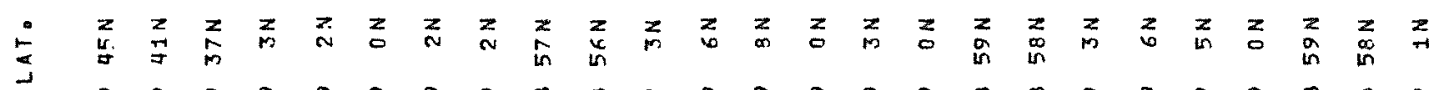
荡

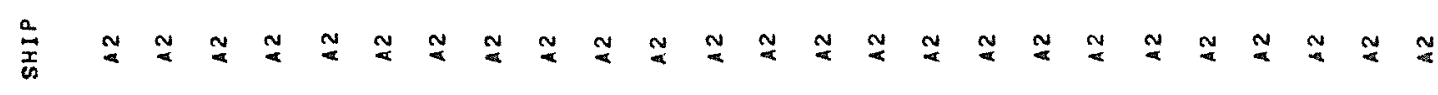

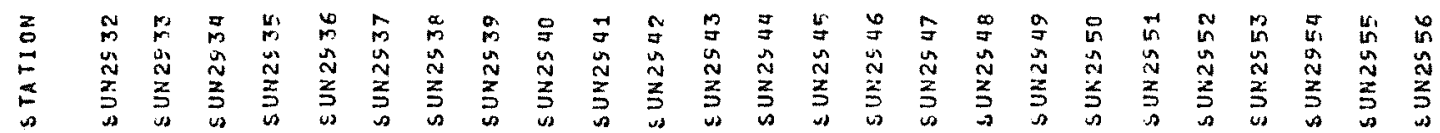


0
0
0
0
0
0
$\vdots$
0
0
0
0
2
0
0
0
-1

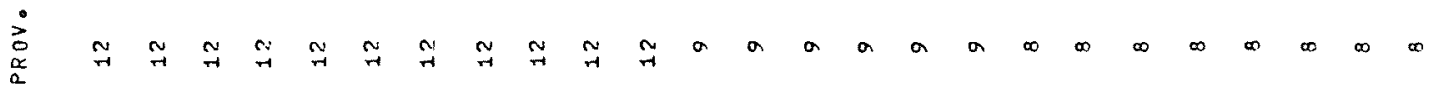

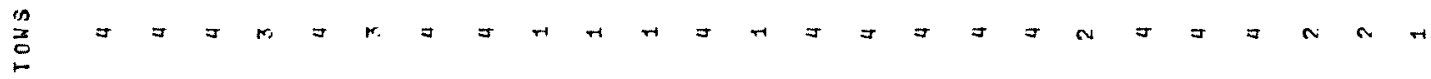

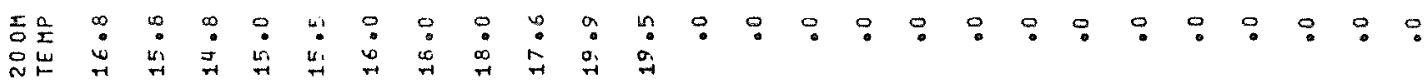

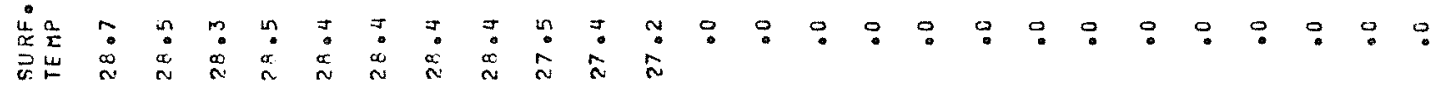

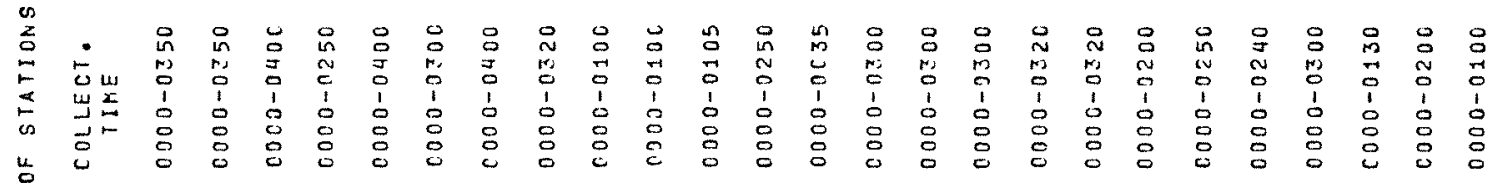

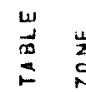

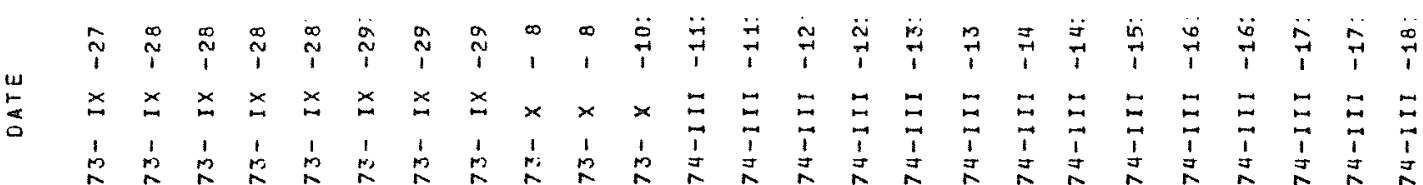

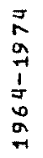

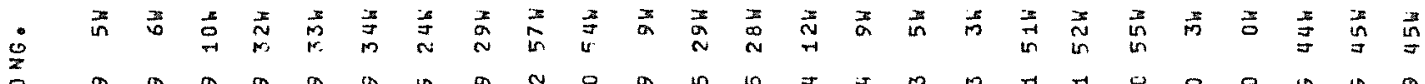
5

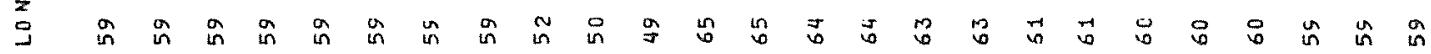

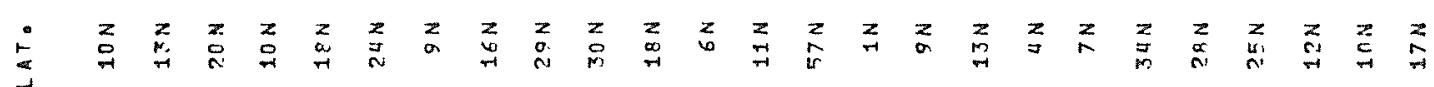

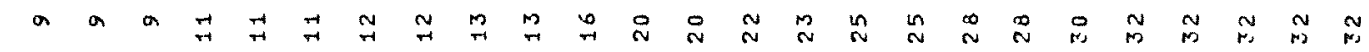

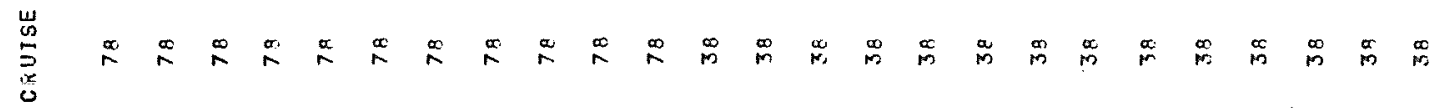

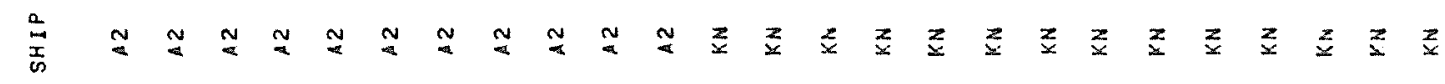

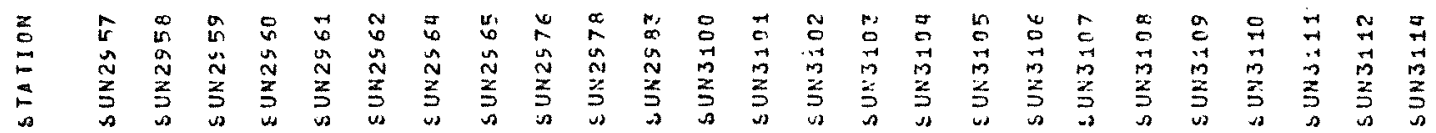




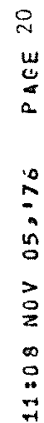

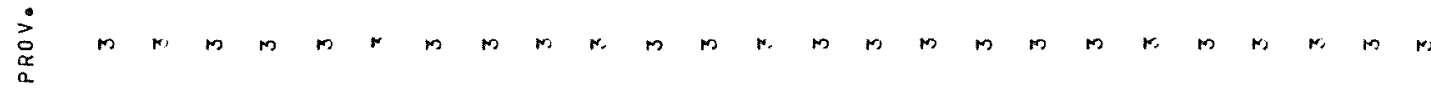

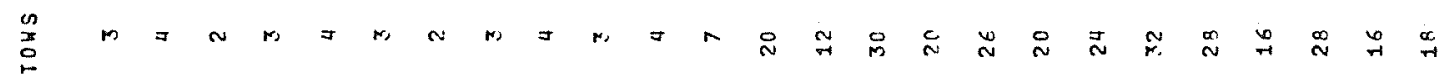

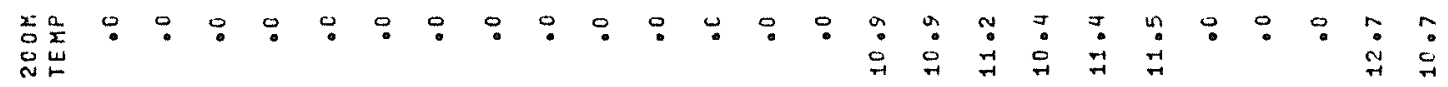

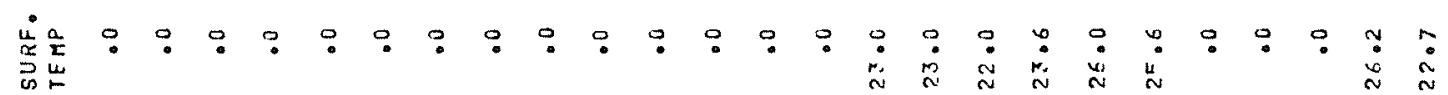

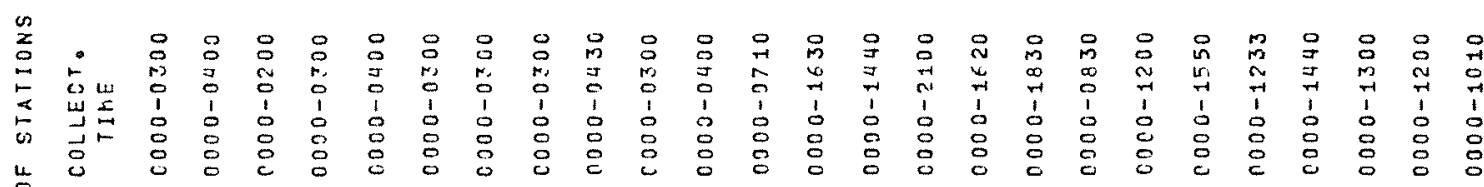

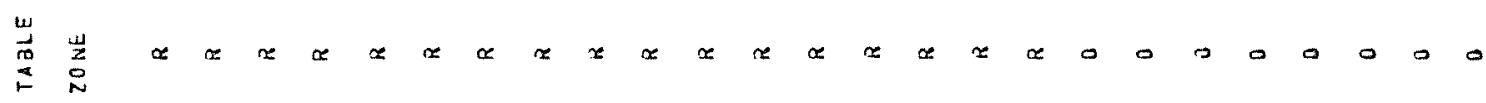

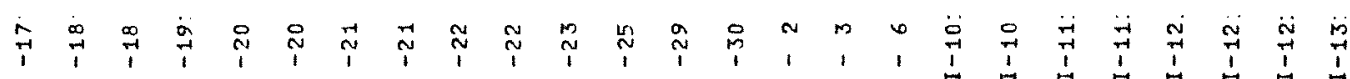

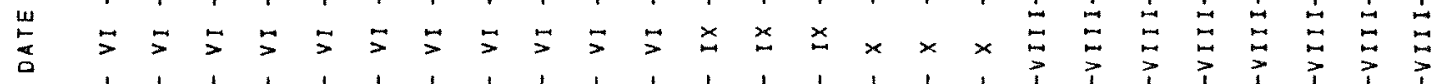

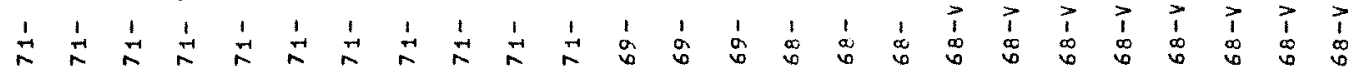

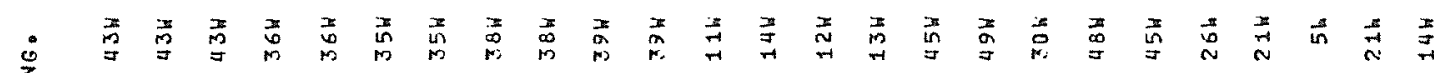

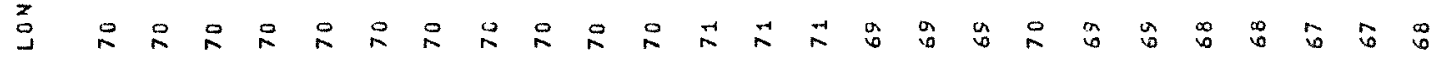

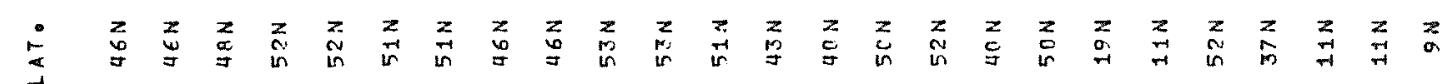

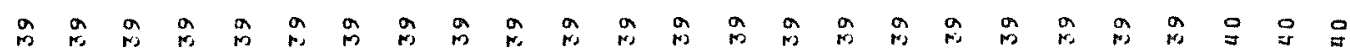

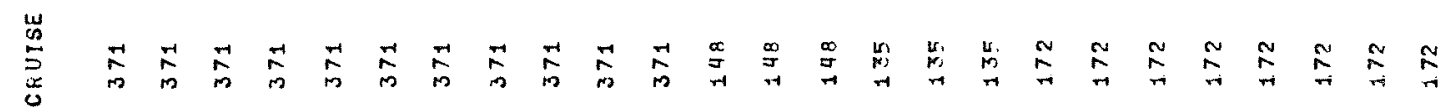

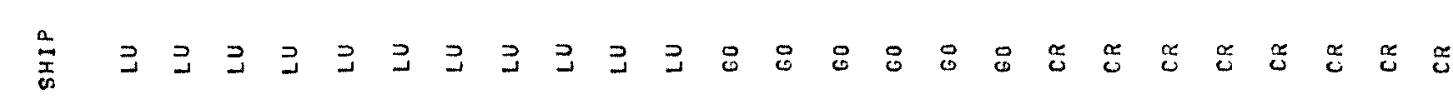

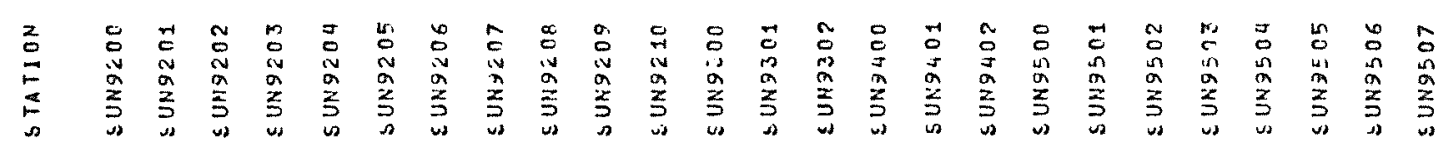


II -22

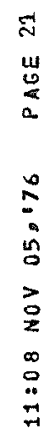

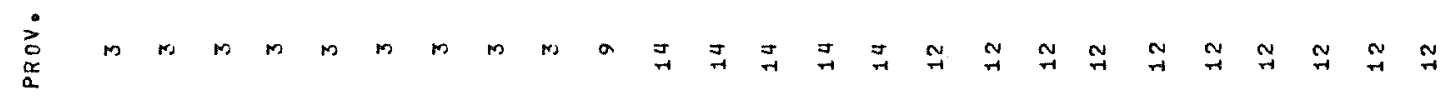

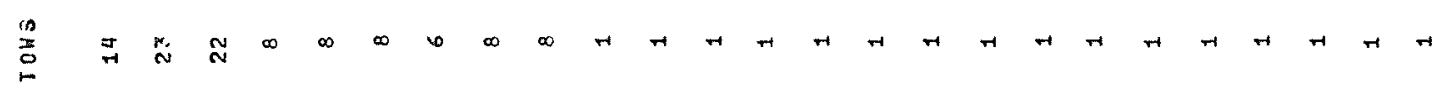

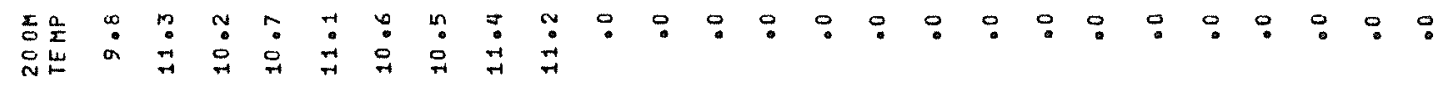

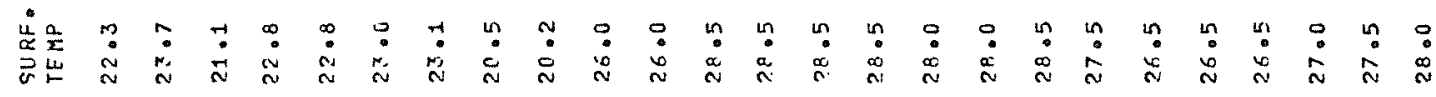

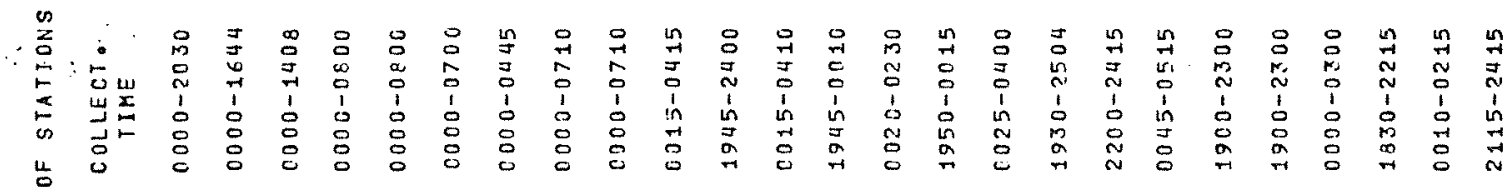

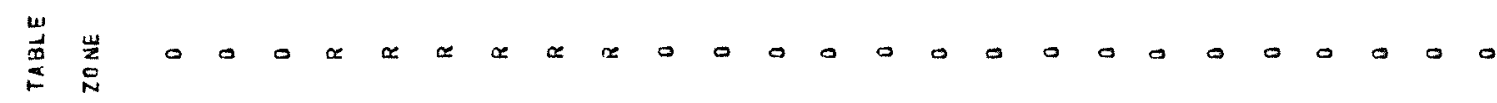

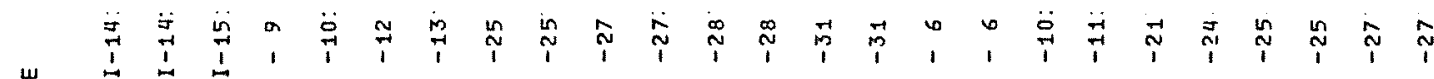

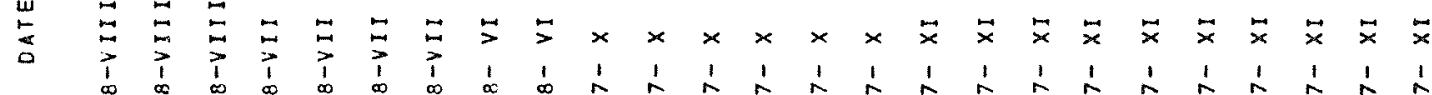

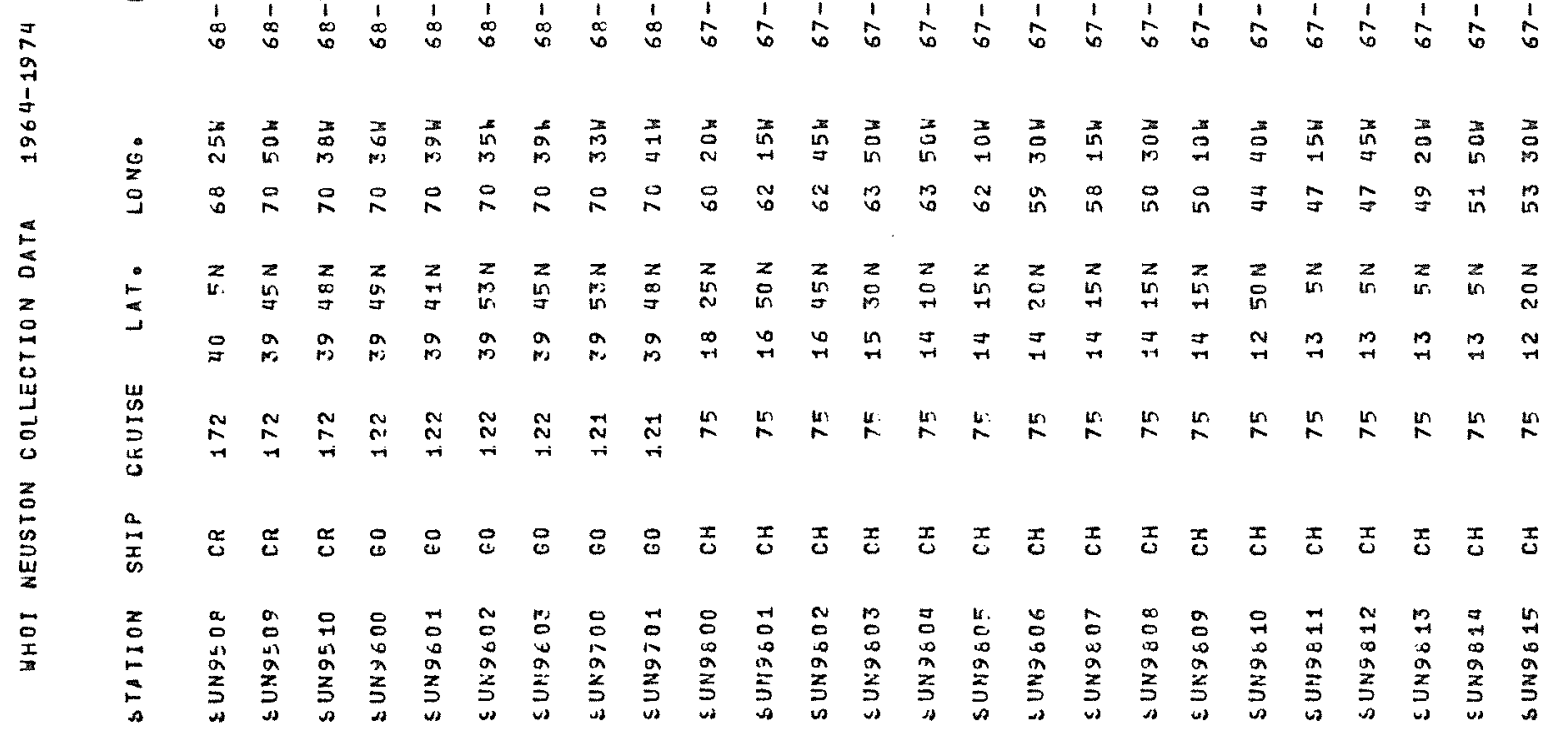




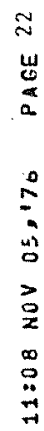

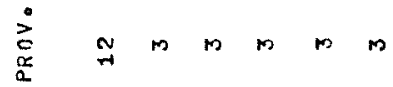

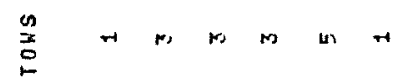

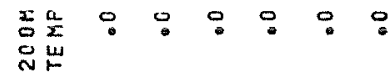

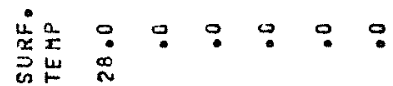

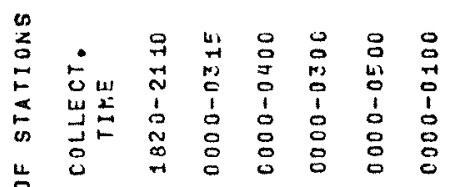

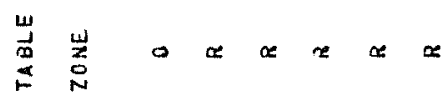

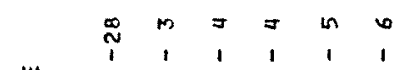

$$
\begin{aligned}
& \text { 岁 }
\end{aligned}
$$

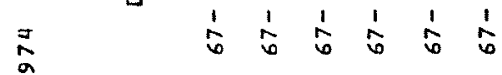

$$
\begin{aligned}
& \underset{5}{5}
\end{aligned}
$$

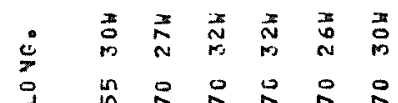

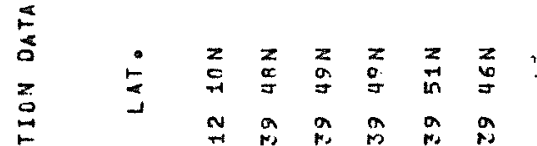

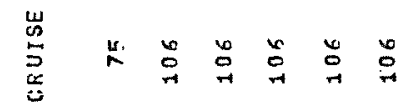

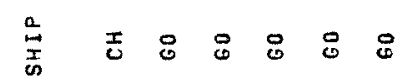

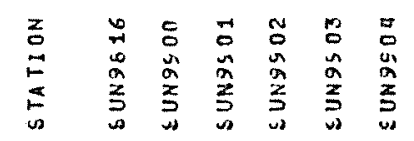


III-I

Appendix 3

Derivation and Coordinates of Atlantic Ocean Pelagic Faunal Boundaries. 


\section{Atlantic Subarctic Region}

The arctic-subarctic boundary is drawn following Dunbar (1951). This boundary marks the southern limit of pure polar basin water. The region is bounded on the south by the North Atlantic Temperate Region. So far, the Atlantic Subarctic Region has not been divided into provinces.

\section{North Atlantic Temperate Region}

The boundary between the Atlantic Subarctic Region and the North Atlantic Temperate Region follows for the most part the oceanic Polar Front (Dietrich 1964). The westernmost segment (that abutting the Slope Water, Province 3) follows the $200-\mathrm{m}$ isotherm for $9{ }^{\circ} \mathrm{C}$ which, according to Worthington (1964), separates subarctic Labrador-Coastal water from the temperate Slope Water. The adjoining segment (that abutting the Northern Gyre, Prov. 2) is formed by the western and northern edges of the Northern Gyre following a North Atlantic circulation diagram of Worthington (in press, Fig. 42). These limits correspond closely to the appropriate parts of the oceanic Polar Front (Dietrich 1964) and of the $200-\mathrm{m}$ isotherm for $9^{\circ} \mathrm{C}$. The segment forming the northern part of the western boundary of the Azores-Britain Province (Prov. 4) is a part of the oceanic Polar Front as drawn by Dietrich (1964). This segment ends at about $59^{\circ} \mathrm{N}, 22^{\circ} \mathrm{W}$, where the Polar Front becomes weak and untraceable. Because the 200-m isotherm for $9^{\circ} \mathrm{C}$ determines or is indistinguishable from the subarctic-temperate boundary all the 
way from the $200-\mathrm{m}$ isobath off Nova Scotia to $59^{\circ} \mathrm{N}, 22^{\circ} \mathrm{W}$, we arbitrarily use that isotherm for making the easternmost segment of the boundary, from $59^{\circ} \mathrm{N}, 22^{\circ} \mathrm{W}$ to $60^{\circ} \mathrm{N}, 5^{\circ} \mathrm{W}$, where the isotherm intersects the $200-\mathrm{m}$ isobath north of Scotland and just south of the Shetland-Faeroes Ridge.

The boundary between the Atlantic Subarctic Region and the North Atlantic Temperate Region is described by the following points (beginning at the 200-m isobath south of Nova Scotia and ending at the 200-m isobath north of Scotland): $42^{\circ} 50^{\circ} \mathrm{N}, 64^{\circ} \mathrm{W} ; 42^{\circ} 45^{\prime} \mathrm{N}, 61^{\circ} \mathrm{W} ; 43^{\circ} 25^{\prime} \mathrm{N}$, $57^{\circ} 30^{\prime} \mathrm{W} ; 42^{\circ} 50^{\prime} \mathrm{N}, 56^{\circ} \mathrm{W} ; 43^{\circ} 15^{\prime} \mathrm{N}, 54^{\circ} 30^{\prime} \mathrm{W} ; 41^{\circ} 30^{\prime} \mathrm{N}, 50^{\circ} \mathrm{W} ; 41^{\circ} \mathrm{N}, 48^{\circ} 45^{\prime} \mathrm{W} ; 4^{\circ} 20^{\prime} \mathrm{N}$, $46^{\circ} \mathrm{W} ; 47^{\circ} \mathrm{N}, 42^{\circ} \mathrm{W} ; 49^{\circ} \mathrm{N}, 43^{\circ} \mathrm{W} ; 51^{\circ} 45^{\prime} \mathrm{N}, 44^{\circ} \mathrm{W} ; 49^{\circ} 20^{\prime} \mathrm{N}, 34^{\circ} \mathrm{W}$; $51^{\circ} \mathrm{N}, 33^{\circ} 45^{\prime} \mathrm{W} ; 52^{\circ} \mathrm{N}, 32^{\circ} \mathrm{W} ; 50^{\circ} 45^{\prime} \mathrm{N}, 30^{\circ} \mathrm{W} ; 50^{\circ} \mathrm{N}, 27^{\circ} 45^{\circ} \mathrm{W} ; 52^{\circ} 30^{\prime} \mathrm{N}$, $26^{\circ} 15^{\prime} \mathrm{W} ; 55^{\circ} \mathrm{N}, 23^{\circ} 30^{\prime} \mathrm{W} ; 59^{\circ} \mathrm{N}, 22^{\circ} 30^{\prime} \mathrm{W} ; 59^{\circ} 30^{\prime} \mathrm{N}, 14^{\circ} \mathrm{W}$; and $60^{\circ} \mathrm{N}, 5^{\circ} \mathrm{W}$.

The North Atlantic Temperate Region is bounded on the south by the North Atlantic Subtropical Region.

The North Atlantic Temperate Region is divided into Slope Water (Prov. 3), Eastern Gyre (Prov. 2), Azores-Britain Province (Prov. 4), Mediterranean Outflow (Prov. 5), and Western and Eastern Mediterranean Seas (Prov. 6 and 7).

The Slope Water is the area to which Iselin (1936) applied this name. Its northern and southern boundaries are the regional boundaries already described. Its eastern limit is set by the southwestern limits of the Eastern Gyre (Worthington in press, Fig. 42). 
The Mediterranean Outflow corresponds to the area in the open Atlantic in which the influence of Mediterranean Water is strongest and is drawn from a plot of salinity in the North Atlantic at $10^{\circ} \mathrm{C}$ (Worthington in press, Fig. 25). (It is at this surface that the Mediterranean water is most conspicuous.) The isohaline for $35.7 \%$, the central isohaline of a bundle of isohalines limiting the pool of most saline water, is used.

The Azores-Britain Province (Prov. 4) is formed by exclusion; its boundaries are determined by the boundaries of its neighbors.

The Strait of Gibraltar and the islands of Sardinia and Corsica determine the limits of the Western and Eastern Mediterranean Seas.

The boundary between the Slope Water (Province 3) and the Northern Gyre (Prov. 4) is described by the points $41^{\circ} \mathrm{N}, 48^{\circ} 45^{\prime} \mathrm{W}$ and $39^{\circ} 40^{\prime} \mathrm{N}, 47^{\circ} 20^{\prime} \mathrm{W}$.

The boundary between the Northern Gyre and the Azores-Britain Province (Prov. 4) is described by the following points: $38^{\circ} 40^{\prime} \mathrm{N}$, $39^{\circ} 25^{\prime} \mathrm{W} ; 43^{\circ} \mathrm{N}, 35^{\circ} \mathrm{W} ; 47^{\circ} \mathrm{N}, 33^{\circ} \mathrm{W}$; and $49^{\circ} 20^{\prime} \mathrm{N}, 34^{\circ} \mathrm{W}$.

The boundary between the Azores-Britain Province and the Mediterranean Outflow (Prov. 5) is described by the following points: $35^{\circ} \mathrm{N}, 20^{\circ} \mathrm{W} ; 42^{\circ} 45^{\prime} \mathrm{N}, 17^{\circ} 15^{\prime} \mathrm{W} ; 46^{\circ} \mathrm{N}, 12^{\circ} 40^{\prime} \mathrm{W}$; and $47^{\circ} \mathrm{N}, 5^{\circ} 15^{\prime} \mathrm{W}$. The Mediterranean Outflow is separated from the Western Mediterranean Sea (Prov. 6) by the sill in the Strait of Gibraltar (Punta de 1a Paloma, $36^{\circ} 03^{\prime} \mathrm{N}, 5^{\circ} 43^{\prime} \mathrm{W}$ to Punta al Boassa, $\left.35^{\circ} 50^{\prime} \mathrm{N}, 5^{\circ} 41^{\prime} \mathrm{W}\right)$. 
The Eastern Mediterranean Sea (Prov. 7) is separated from the Western Mediterranean Sea by Corsica and Sardinia, the shallows between them, and their natural extensions north and south following the shoal water $\left(43^{\circ} 45^{\prime} \mathrm{N}\right.$, $10^{\circ} \mathrm{E}$ to $43^{\circ} \mathrm{N}, 9^{\circ} 25^{\prime} \mathrm{E}$ and $38^{\circ} 50^{\prime} \mathrm{N}, 8^{\circ} 52^{\prime} \mathrm{E}$ to $\left.37^{\circ} 13^{\prime} \mathrm{N}, 9^{\circ} 13^{\prime} \mathrm{E}\right)$.

\section{North Atlantic Subtropical Region}

The boundary between the North Atlantic Temperate Region and the North Atlantic Subtropical Region runs along the northern edges of the Sargasso Sea and the North African Subtropical Sea, the eastern Atlantic counterpart of the Sargasso Sea. The western segment follows the shoreward edge of the Gulf Stream and is determined by the $200-\mathrm{m}$ isotherm for $15^{\circ} \mathrm{C}$ (Worthington 1964). The central segment we take from the southern edge of the Northern Gyre (Worthington in press, Fig. 42). The eastern segment is determined by the northern limit in late winter of water as warm as $15^{\circ} \mathrm{C}$ following Worthington (personal communication), who suggests that this is a suitable criterion for fixing the northern edge of the subtropical sea in the eastern North Atlantic, and drawn after him (in press, Fig. 28).

The boundary between the North Atlantic Temperate Region and the North Atlantic Subtropical Region is described by the following points (beginning at the 200-m isobath off Cape Hatteras and ending at the 200-isobath off western Morocco): $35^{\circ} 20^{\prime} \mathrm{N}, 75^{\circ} \mathrm{W} ; 38^{\circ} 20^{\prime} \mathrm{N}, 67^{\circ} 30^{\prime} \mathrm{W}$, $38^{\circ} 15^{\prime} \mathrm{N} ; 65^{\circ} 30^{\prime} \mathrm{W} ; 40^{\circ} 50^{\prime} \mathrm{N}, 61^{\circ} 25^{\prime} \mathrm{W} ; 40^{\circ} 35^{\prime} \mathrm{N}, 53^{\circ} 30^{\prime} \mathrm{W} ; 38^{\circ} 45^{\prime} \mathrm{N}, 48^{\circ} 45^{\prime} \mathrm{W} ;$ $39^{\circ} 40^{\prime} \mathrm{N}, 47^{\circ} 20^{\prime} \mathrm{W} ; 37^{\circ} \mathrm{N}, 41^{\circ} \mathrm{W} ; 38^{\circ} 40^{\prime} \mathrm{N}, 39^{\circ} 25^{\prime} \mathrm{W} ; 37^{\circ} 20^{\prime} \mathrm{N}, 37^{\circ} \mathrm{W} ; 37^{\circ} 05^{\prime} \mathrm{N}$, $34^{\circ} 30^{\prime} \mathrm{W} ; 36^{\circ} \mathrm{N}, 31^{\circ} 10^{\circ} \mathrm{W} ; 35^{\circ} 15^{\prime} \mathrm{N}, 26^{\circ} 35^{\prime} \mathrm{W} ; 35^{\circ} \mathrm{N}, 20^{\circ} \mathrm{W} ;$ and $32^{\circ} 50^{\prime} \mathrm{N}, 10^{\circ} \mathrm{W}$. 
The North Atlantic Subtropical Region is bounded on the south by the Atlantic Tropical Region and in the southeast by the Mauritanian Upwelling.

The North Atlantic Subtropical Region is divided into four provinces by north-south and east-west running boundaries. The north-south running boundary is the eastern edge of the Sargasso Sea; to the east 1ies the North African Subtropical Sea. The east-west running boundary is Wuist's "nordliche subtropische Konvergenz" (Wiist 1928) and separates both the Sargasso Sea and the North African Subtropical Sea into northern and southern parts. There is evidence (summarized in Backus et al. 1969) that Wist's feature is the same phenomenon as the so-called "thermal fronts" of the Sargasso Sea (Voorhis and Hersey 1964, Katz 1969) and that it results from converging surface drifts in the transition region between westerlies and northeast trades.

The Northern and Southern Sargasso Seas (Prov. 8 and 9) are separated from the Northern and Southern North African Subtropical Seas (Prov. 10 and 11) by a boundary described by the following points: $37^{\circ} \mathrm{N}, 41^{\circ} \mathrm{W} ; 33^{\circ} \mathrm{N}$, $44^{\circ} \mathrm{W}$; and $22^{\circ} 30^{\prime} \mathrm{N}, 44^{\circ} \mathrm{W}$.

The Northern Sargasso and Northern North African Subtropical Seas are separated from the Southern Sargasso and Southern North African Subtropical Seas by a boundary described by the following points (beginning at the north end of Little Bahama Bank and ending at the 200-m isobath on the coast of Africa east of the Canary Islands): $27^{\circ} 25^{\prime} \mathrm{N}, 78^{\circ} 45^{\prime} \mathrm{W}$; $26^{\circ} \mathrm{N}, 72^{\circ} \mathrm{W} ; 27^{\circ} 30^{\prime} \mathrm{N}, 69^{\circ} \mathrm{W} ; 27^{\circ} 30^{\prime} \mathrm{N}, 65^{\circ} \mathrm{W} ; 28^{\circ} \mathrm{N}, 61^{\circ} \mathrm{W} ; 29^{\circ} 45^{\prime} \mathrm{N}, 56^{\circ} \mathrm{W} ; 30^{\circ} \mathrm{N}$, $52^{\circ} 30^{\prime} \mathrm{W} ; 34^{\circ} \mathrm{N}, 47^{\circ} 30^{\circ} \mathrm{W} ; 33^{\circ} \mathrm{N}, 44^{\circ} \mathrm{W} ; 32^{\circ} \mathrm{N}, 40^{\circ} \mathrm{W} ; 29^{\circ} 30^{\prime} \mathrm{N}, 35^{\circ} \mathrm{W} ; 29^{\circ} \mathrm{N}$, $30^{\circ} \mathrm{W} ; 32^{\circ} \mathrm{N}, 22^{\circ} 30^{\prime} \mathrm{W}$, and $27^{\circ} 30^{\prime} \mathrm{N}, 13^{\circ} 35^{\circ} \mathrm{W}$. 
South Atlantic Subtropical Region

The South Atlantic Subtropical Region is much like its northern hemisphere counterpart. This subtropical sea is formed by a more or less triangular, anti-cyclonic gyre whose base lies off Brazil and whose apex almost reaches Southwest Africa. An Atlantic Ocean temperature plot for $200 \mathrm{~m}$ (Wiist and Defant 1936) shows the gyral as having a maximum temperature at $200 \mathrm{~m}$ of about $18^{\circ}$ or $19^{\circ} \mathrm{C}$ near its base and cooling more or less regularly towards its sides. We use the $13^{\circ} \mathrm{C}$ isotherm at $200 \mathrm{~m}$ for limiting it. Earlier, we used the $15^{\circ} \mathrm{C}$ isotherm for $200 \mathrm{~m}$ (Backus et at. 1970). However, the $13^{\circ} \mathrm{C}$ isotherm, which follows the $15^{\circ}$ one, but at a greater distance from the center of the gyre, delimits a region that better fits its neighbors. Along the northeast boundary the $13^{\circ}$ isotherm nearly coincides with the boundary that we draw for limiting the tropical Guinean Province (Prov. 16) using a dissolved oxygen criterion. To the south the $13^{\circ} \mathrm{C}$ isotherm more nearly coincides with the South Atlantic Subtropical Convergence, the logical southern limit of the South Atlantic subtropical sea.

The South Atlantic Subtropical Region is bounded by a line described by the following points (beginning at the $200-\mathrm{m}$ isobath off easternmost Brazil and going clockwise to the 200-m isobath off Rio de la Plata): $5^{\circ} 45^{\prime} \mathrm{S}, 34^{\circ} 50^{\prime} \mathrm{W} ; 7^{\circ} 15^{\prime} \mathrm{S}, 30^{\circ} \mathrm{W} ; 9^{\circ} \mathrm{S}, 27^{\circ} 30^{\prime} \mathrm{W}$; $5^{\circ} 45^{\prime} \mathrm{S}, 24^{\circ} 30^{\prime} \mathrm{W} ; 11^{\circ} \mathrm{S}, 16^{\circ} 45^{\prime} \mathrm{W} ; 16^{\circ} \mathrm{S}, 8^{\circ} \mathrm{W} ; 18^{\circ} \mathrm{S}, 3^{\circ} \mathrm{W} ; 22^{\circ} 30^{\prime} \mathrm{S}$, $5^{\circ} \mathrm{E} ; 25^{\circ} \mathrm{S}, 8^{\circ} \mathrm{E} ; 28^{\circ} \mathrm{S}, 11^{\circ} \mathrm{E} ; 33^{\circ} \mathrm{S}, 12^{\circ} \mathrm{E} ; 36^{\circ} 30^{\prime} \mathrm{S}, 9^{\circ} \mathrm{E} ; 34^{\circ} \mathrm{S}$, $1^{\circ} 30^{\prime} \mathrm{W} ; 33^{\circ} \mathrm{S}, 7^{\circ} 30^{\prime} \mathrm{W} ; 33^{\circ} 30^{\prime} \mathrm{S}, 12^{\circ} \mathrm{W} ; 34^{\circ} 30^{\prime} \mathrm{S}, 22^{\circ} 30^{\prime} \mathrm{W} ; 37^{\circ} \mathrm{S}$, 
$25^{\circ} \mathrm{W} ; 37^{\circ} \mathrm{S}, 32^{\circ} 30^{\prime} \mathrm{W} ; 31^{\circ} 30^{\prime} \mathrm{S}, 37^{\circ} 30^{\prime} \mathrm{W}$; and $35^{\circ} \mathrm{S}, 52^{\circ} 30^{\prime} \mathrm{W}$.

The South Atlantic Subtropical Region is bounded on the north by the Atlantic Tropical Region. It has not yet been divided into provinces.

\section{Atlantic Tropical Region}

The boundary between the North Atlantic Subtropical Region and the Atlantic Tropical Region is drawn one way in the west, another way in the east. In the west, the boundary follows the southern edge of the Sargasso Sea, which is characterized in the upper few hundred meters by a temperature close to $18^{\circ} \mathrm{C}$ and a salinity between 36.4 and $36.6 \%$. A plot of salinity at 300-m (Worthington in press, Fig. 33) shows the extent of the sea well and is followed here.

In the east, the subtropical sea extends farther to the south. We draw the boundary to follow the line of change between tropical and subtropical temperature' regimens in the upper few hundred meters of the water column.

We examined the point of change in a meridional section at about $40^{\circ} \mathrm{W}$ (Backus et $\alpha 2$. 1965) and found that it coincided with the meeting of North Atlantic Central Water and South Atlantic Central Water. Furthermore it appeared that the $200-\mathrm{m}$ isotherm for $14^{\circ} \mathrm{C}$, which traverses the Atlantic between northwest Africa and northern South America, marked the point of change. This isotherm corresponds reasonably well with the position of the boundary between the two water masses as shown by 
Sverdrup et $\alpha$ I. (1946), and we use it to draw the southern boundary of the North African Subtropical Sea (Prov. 11), forming the eastern part of the tropical-subtropical boundary.

The western and eastern portions of the tropical-subtropical boundary are connected arbitrarily by a more or less north-south segment that follows the mid-Atlantic Ridge:

The boundary between the North Atlantic Subtropical Region and the Atlantic Tropical Region is described by following points (beginning at the 200-m isobath at Anguilla Bank and ending at the Mauritanian Upwelling): $18^{\circ} 35^{\prime} \mathrm{N}, 62^{\circ} 50^{\prime} \mathrm{W} ; 19^{\circ} \mathrm{N}, 47^{\circ} 15 \mathrm{l} ; 12^{\circ} 45^{\prime} \mathrm{N}, 44^{\circ} 40^{\prime} \mathrm{W} ; 12^{\circ} 45^{\prime} \mathrm{N}, 43^{\circ} 30^{\prime} \mathrm{W}$; $16^{\circ} 30^{\prime} \mathrm{N}, 34^{\circ} \mathrm{W}$; and $18^{\circ} 20^{\prime} \mathrm{N}, 22^{\circ} 30^{\prime} \mathrm{W}$.

The Atlantic Tropical Region is bounded on the south by the South Atlantic Subtropical Region.

In the very northwest the Atlantic Tropical Region abuts the Northern Sargasso Sea. The two are separated by a line running from the northern end of Little Bahama Bank $\left(27^{\circ} 25^{\prime} \mathrm{N}, 78^{\circ} 45^{\prime} \mathrm{W}\right)$ to a point on the $200-\mathrm{m}$ isobath off Jupiter Inlet $\left(27^{\circ} 05^{\prime} \mathrm{N}, 79^{\circ} 55^{\prime} \mathrm{W}\right)$.

On the extreme southeast the Atlantic Tropical Region is bounded by a line described by the points $22^{\circ} 30^{\prime} \mathrm{S}, 5^{\circ} \mathrm{E}$ and $23^{\circ} \mathrm{S}, 13^{\circ} 30^{\prime} \mathrm{E}$.

The Atlantic Tropical Region is divided into five provinces. Two of these, the Guinean and Amazonian Provinces (Prov. 16 and 15) are traversed by the geographic equator and occupy most of the open-ocean part of the region between Africa and South America. A third, the Lesser Antillean Province (Prov. 12), is somewhat transitional between the aforementioned equatorial seas and the subtropical Sargasso Sea. The Lesser Antillean Province in turn is physically and faunally related to the 
Caribbean Sea (Prov. 14), a natural hydrographic extension of which is the Straits of Florida (Prov. 23).

The western equatorial province, the Amazonian, is somewhat warmer, saltier, has more dissolved oxygen, and is less productive than the eastern equatorial province, the Guinean.

We draw the boundary between these two provinces on the basis of the distribution of dissolved oxygen, thinking that this property is apt to be most significant. There is a marked oxygen minimum layer in the equatorial eastern Atlantic, and this lies at about $400 \mathrm{~m}$. Reference to the horizontal distribution of dissolved oxygen at this level (Wattenberg 1939) suggests that the east-west gradient is sharpest at about $2 \mathrm{cc} / 1$, and we have used this isopleth to draw the boundary. The isopleth makes a great eastward involution at the equator; we have bridged this in drawing the westward boundary of the province. No doubt more information will show that the Guinean Province as we have drawn it needs to be subdivided.

The $14^{\circ} \mathrm{C}$ isotherm for $200 \mathrm{~m}$, which marks the boundary between tropical and subtropical regions in the east (see above), is a provincial boundary in the west, setting apart the Lesser Antillean and Amazonian Provinces.

The Straits of Florida (Prov. 23) is separated from the Caribbean Sea by a line drawn from a point on the $200-\mathrm{m}$ isobath off Cabo Catoche, Yucatan $\left(21^{\circ} 40^{\prime} \mathrm{N}, 86^{\circ} 25^{\prime} \mathrm{W}\right)$ to a point on the $200-\mathrm{m}$ isobath off Cabo San Antonio, Cuba $\left(21^{\circ} 50^{\prime} \mathrm{N}, 85^{\circ} 55^{\prime} \mathrm{W}\right)$. 
The Caribbean Sea (Prov. 14) has natural topographic limits as already noted.

The Lesser Antillean Province (Prov. 12) is separated from the Amazonian Province (Prov. 15) by a line connecting the points $9^{\circ} \mathrm{N}, 59^{\circ} \mathrm{W}$; $12^{\circ} 30^{\prime} \mathrm{N}, 50^{\circ} \mathrm{W}$; and $12^{\circ} 45^{\prime} \mathrm{N}, 43^{\circ} 30^{\prime} \mathrm{W}$. The Amazonian Province is separated from the Guinean Province (Prov. 16) by a line connecting the points $15^{\circ} 10^{\prime} \mathrm{N}, 37^{\circ} 30^{\prime} \mathrm{W} ; 8^{\circ} 30^{\prime} \mathrm{N}, 35^{\circ} \mathrm{W} ; 6^{\circ} \mathrm{N}, 28^{\circ} \mathrm{W} ; 0^{\circ}, 21^{\circ} \mathrm{W}$; and $5^{\circ} 45^{\prime} \mathrm{S}, 24^{\circ} 30^{\prime} \mathrm{W}$.

The Guinean Province is subdivided by a line connecting the points $6^{\circ} \mathrm{N}, 28^{\circ} \mathrm{W} ; 6^{\circ} \mathrm{N}, 20^{\circ} \mathrm{W} ; 3^{\circ} \mathrm{N}, 14^{\circ} \mathrm{W} ; 2^{\circ} \mathrm{N}, 5^{\circ} \mathrm{W} ; 5^{\circ} \mathrm{N}, 1^{\circ} \mathrm{W} ; 2^{\circ} 30^{\prime} \mathrm{S}, 0^{\circ} ;$ $2^{\circ} 30^{\prime} \mathrm{S}, 7^{\circ} 30^{\prime} \mathrm{W} ; 2^{\circ} \mathrm{S}, 14^{\circ} \mathrm{W}$; and $0^{\circ}, 21^{\circ} \mathrm{W}$.

\section{Mauritanian Upwelling}

For setting this region's limits we follow the suggestion of Wooster and Reid (1963) that the zone of upwelling is characterized by a wide annual variation in surface temperature. From a chart of the same (Böhnecke 1936), we have taken as our boundary the isopleth for a variation of $5^{\circ} \mathrm{C}$, the outermost of a bundle of isopleths surrounding the upwelling area.

The Mauritanian Upwelling Region is divided into northern and southern provinces by the eastern extremity of the $14^{\circ} \mathrm{C}$ isotherm for $200 \mathrm{~m}$. As indicated above, this isotherm labels approximately the meeting of South Atlantic Central and North Atlantic Central Water Masses, and it appears that two sorts of water are upwelling in this region. 
The Mauritanian Upwelling is separated from the North Atlantic Subtropical Region and the Atlantic Tropical Region by a boundary described by the following points: $22^{\circ} 20^{\prime} \mathrm{N}, 17^{\circ} 30^{\prime} \mathrm{W} ; 20^{\circ} 40^{\prime} \mathrm{N}, 20^{\circ} \mathrm{W} ; 20^{\circ} \mathrm{N}$, $21^{\circ} 12^{\prime} \mathrm{W} ; 18^{\circ} 20^{\prime} \mathrm{N}, 22^{\circ} 30^{\prime} \mathrm{W} ; 15^{\circ} \mathrm{N}, 22^{\circ} 30^{\prime} \mathrm{W} ; 11^{\circ} 15^{\prime} \mathrm{N}, 20^{\circ} \mathrm{W}$; and $10^{\circ} \mathrm{N}$, $17^{\circ} 30^{\prime} \mathrm{W}$.

The Northern Mauritanian Upwelling (Prov. 18) is separated from the Southern Mauritanian Upwelling (Province 19) by a line connecting the following points: $18^{\circ} 20^{\prime} \mathrm{N}, 22^{\circ} 30^{\prime} \mathrm{W}$ and $20^{\circ} \mathrm{N}, 17^{\circ} 40^{\prime} \mathrm{W}$.

\section{Gulf of Mexico}

The Gulf of Mexico is separated from the Straits of Florida (Prov. 23) by a line drawn from a point on the 200-m isobath off Cabo Catoche, Yucatan $\left(22^{\circ} \mathrm{N}, 86^{\circ} 30^{\prime} \mathrm{W}\right)$ to a point on the $200-\mathrm{m}$ isobath off Dry Tortugas, Florida $\left(24^{\circ} 20^{\prime} \mathrm{N}, 83^{\circ} 08^{\prime} \mathrm{W}\right)$. The Gulf of Mexico is undivided and can be viewed as both region and province. 


\section{Literature Cited}

Backus, R. H., J. E. Craddock, R. L. Haedrich, and D. L. Shores. 1969. Mesopelagic fishes and thermal fronts in the western Sargasso Sea. Mar. Biol. 3: 87-106.

Backus, R. H., J. E. Craddock, R. L. Haedrich and D. L. Shores. 1970. The distribution of mesopelagic fishes in the equatorial and western North Atlantic Ocean. J. Mar. Res. 28: 179-201.

Backus, R. H., G. W. Mead, R. L. Haedrich, and A. W. Ebeling. 1965. The mesopelagic fishes collected during Cruise 17 of the $R / V$ Chain, with a method for analyzing faunal transects. Bull. Mus. Comp. Zoo1. 134: 139-158.

Böhnecke, G. 1936. Temperatur, Salzgehalt und Dichte an der Oberfläche des Atlantischen Ozeans. Wiss. Ergeb. Deutsch. Atlant. Exped. Forsch. Verm. "Meteor" 1925-1927 5 (Atlas): p1. 24.

Dietrich, G. 1964. Oceanic polar front survey in the North Atlantic, In Solid earth and interface phenomena, Research in Geophysics, 2: 291-308. MIT.

Dunbar, M. J. 1951. Eastern arctic waters. Bull. Fish. Res. Bd. Canada, 88: 1-131.

Iselin, C. O'D. 1936. A study of the circulation of the western North Atlantic. Pap. Phys. Oceanogr. Meteoro1. 4: 1-101.

Katz, E. I, 1969, Further study of a front in the Sargasso Sea. Te1lus 21: 259-269.

Sverdrup, H. U., M. W. Johnson, and R. H. Fleming. 1946. The Oceans. Prentice-Ha11. 
Voorhis, A. D. and J. B. Hersey. 1964. Oceanic thermal fronts in the Sargasso Sea. J. Geophy. Res. 69: 3809-3814.

Wattenberg, H. 1939. Die Verteilung des Sauerstoffs im Atlantischen

Ozean. Wiss. Ergeb. Deutsch. Atlant. Exped. Forsch. Verm.

"Meteor" 1925-1927 9(Atlas): p1. 38.

Wooster, W. S. and J. L. Reid, Jr. 1963. Eastern boundary currents,

p. 253-280. In: M. N. Hill [ed.], The sea, vol. 2, Interscience.

Worthington, L. V. 1964. Anomalous conditions in the Slope Water area

in 1959. J. Fish. Res. Bd. Canada 21: 327-333.

Worthington, L. V. In press. On the North Atlantic circulation. Johns Hopkins Oceanographic Studies.

Wist, G. 1928. Der Ursprung der atlantischen Tiefenwasser. Z. Ges. Erdk. Berl., Sonderband zur Hundertjahrfeier: 506-534.

Wist, G., and A. Defant. 1936. Schichtung und Zirkulation des Atlantischen Ozeans. Wiss. Ergeb. Deutsch. Atlant. Exped. Forsch. Verm. "Meteor" 1925-1927: 6(Atlas): pl. 47. 


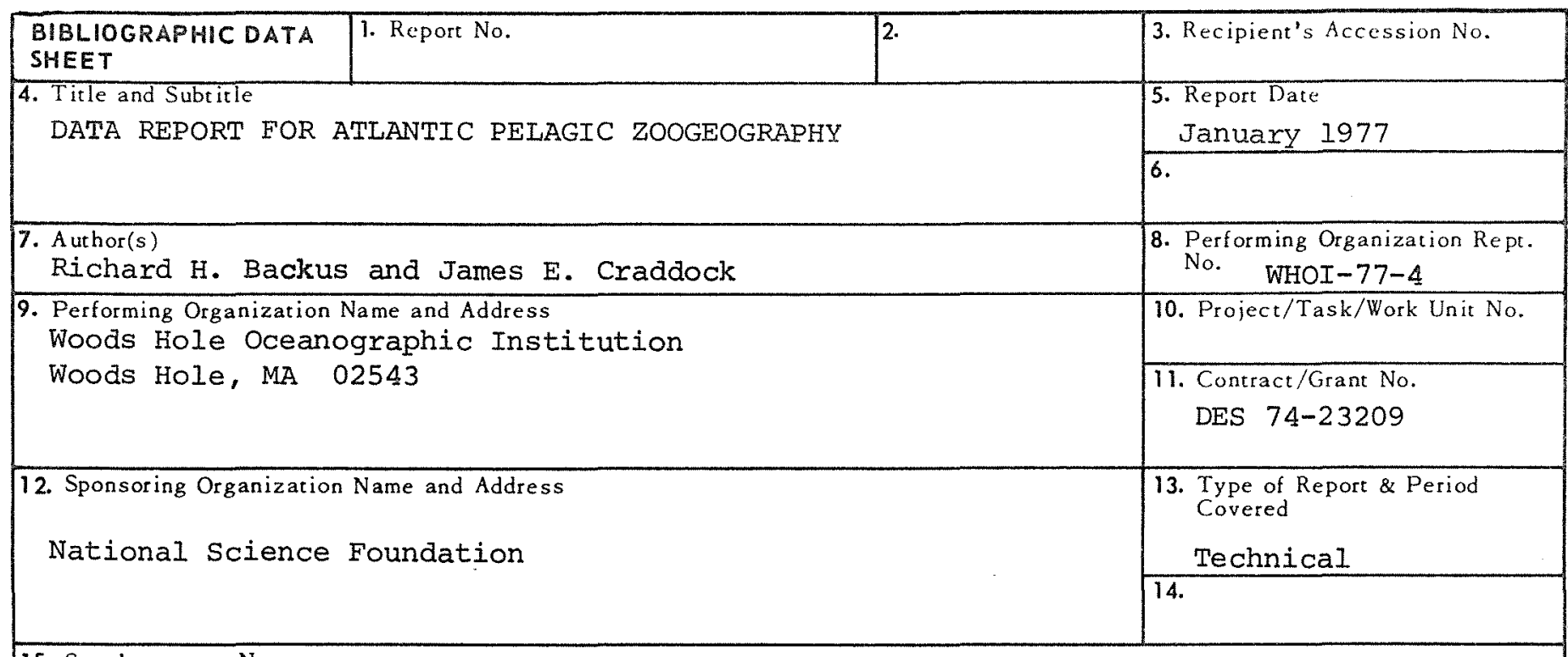

15. Supplementary Notes

16. Abstracts

This report (1) gives station data for midwater-trawl and neuston collections made in the Atlantic Ocean between 1961 and 1974 and (2) gives the geographic coordinates of and explains the derivation of a system of Atlantic faunal boundaries.

17. Key Words and Document Analysis. 17a. Descriptors

1. Atlantic pelagic zoogeography

2. Midwater-trawl and neuston collections

3. Faunal boundaries

17b. Identifiers/Open-Ended Terms

17c. COSATI Field/Group

18. Availability Statement

a sys

Security Class (This

Report)

UNCLASSIFIED

0. Security Class (This

Page

21. No. of Pages 85

22. Price 


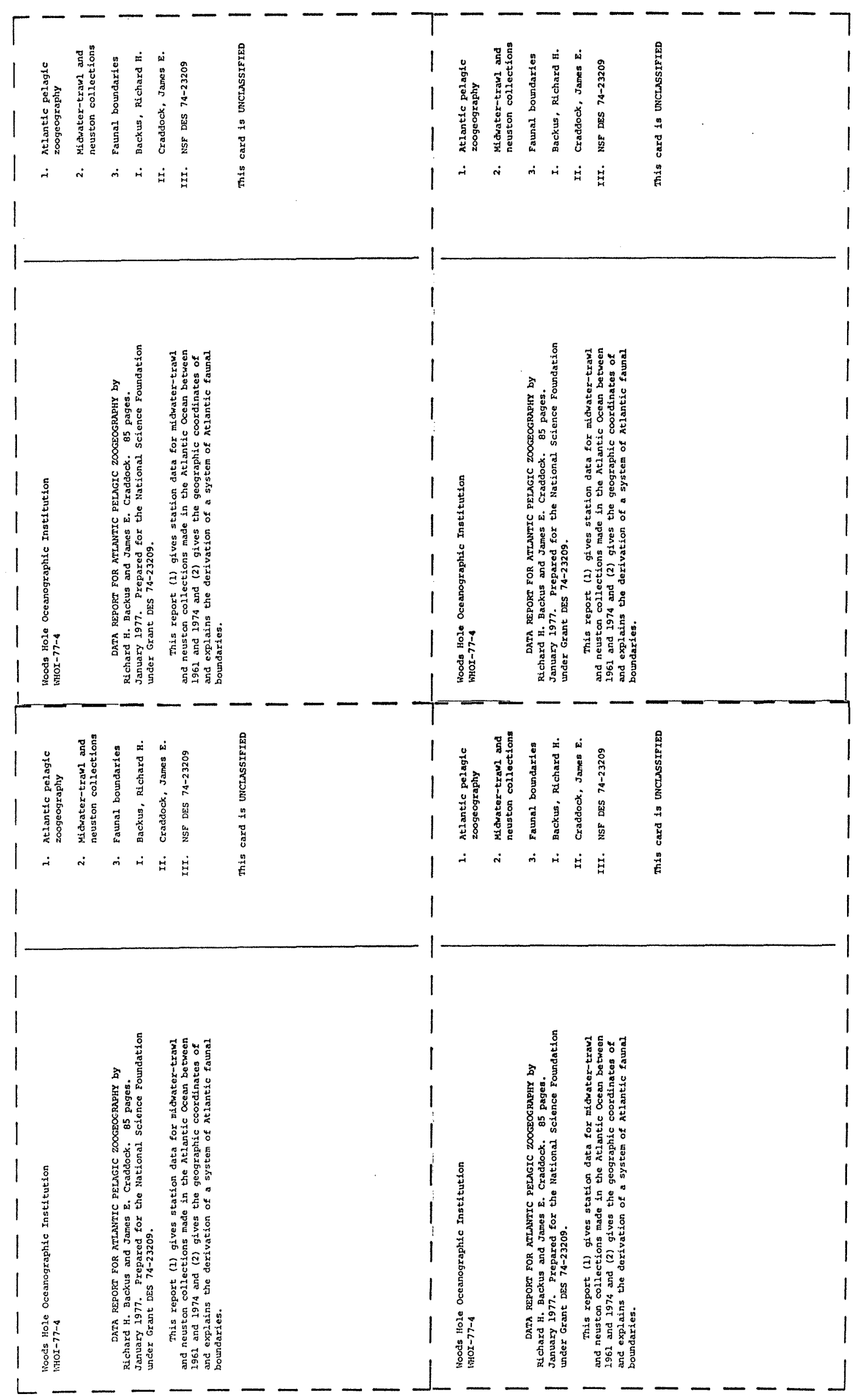

